\title{
Index of Surface-Water Records to December 31, 1963
}

Part 3.-Ohio River Basin

GEOLOGICAL SURVEY CIRCULAR 503 


\title{
Index of Surface-Water Records to December 31, 1963 Part 3.-Ohio River Basin
}

\author{
By B. A. Anderson and C. B. Ham
}

父

Geological Survey Circular 503 
United States Department of the Interior STEWART L. UDALL, SECRETARY

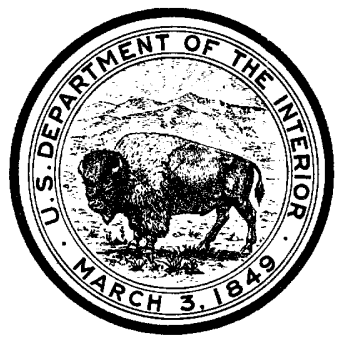

Geological Survey

WILLIAM T. PECORA Director

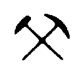

Free on application to the U.S. Geological Survey, Washington, D. C. 20242 


\title{
Index of Surface-Water Records to December 31,1963
}

\author{
Part 3.-Ohio River Basin
}

By B. A. Anderson and C. B. Ham

INTRODUCTION

This report lists the streamflow and reservoir stations in the Ohio River basin for which records have been or are to be published in reports of the Geological Survey for periods through December 31, 1963. It supersedes Geological Survey Circular 383.

Basic data on surface-water supply have been published in an annual series of water-supply papers consisting of several volumes, including one each for the States of Alaska and Hawa11. The area of the other 48 States is divided into 14 parts whose boundaries coincide with certain natural drainage lines. Prior to 1951, the records for the 48 States were published in 14 volumes, one for each of the parts. From 1951 to 1960, the records for the 48 states were published annualiy in 18 volumes, there being 2 volumes each for parts $1,2,3$, and 6 . The boundaries of the various parts are shown on the map in figure 1.

Beginning in 1961, the annual series of water-supply papers on surface-water supply was changed to a 5-year series. Records for the period 1961-65 will be published in a series of water-supply papers using the same 14 part divisions for the 48 states, but most parts will be further subdivided into two or more volumes. In order to meet interim requirements, beginning in 1961, local offices issue reports annually containing streamflow records for the State or states within the local district.

In addition to the continuous-record gaging stations, this index includes crest-stage and low-flow partial-record stations. A continuous-record station is a gaging station on a stream or reservoir for which the discharge, stage, or contents is published on a daily, weekly, or monthly basis for a continuous period of time. A crest-stage partial-record station is a streamflow station for which only the annual maximum discharge is published over a pertod of years for use in floodflow analyses. A low-flow partial-record station is a streamflow station for which only discharge measurements made at base flow, when streamflow is primarily from ground-water storage, are published. Measurements are generally collected over a period of five years or more for use in low-flow analyses. Discharge measurements have been made at many sites and published as miscellaneous measurements. Such measurements are not included in this index except those made in earlier years at sites now classified as low-flow partial-record stations.

An alphabetical list of streams, lakes, and reservoirs is given on pages 49-57.

\section{DOWNSTREAM ORDER}

The stations in this index are listed in the downstream order used in the series of watersupply papers on surface-water supply of the United States since 1951. Starting at the headwater of each stream, all stations are listed in a downstream direction. Tributary streams are indicated by indention and are inserted between main-stem stations in the order in which they enter the main stream. To indicate the rank of any tributary on which a record is available and the stream to which it is immediately tributary, each indention in the listing of stations represents one rank. A stream name, only, is inserted where necessary for the purpose of showing the proper rank or order of tributaries. For some stations on lakes for which the outlet stream is unnamed or which are far removed in tributary rank from the gaged stream, a double indention beyond the preceding entry is used to indicate that each direct tributary step is not shown.

\section{STATION NAMES}

Station names are given in their most recently published form. Parentheses around part of a station name indicate that the enclosed word or words were used in an earlier published name of the station or as an alternate name. Parenthetical explanations are also used to indicate that a stream of a different name is the head of the main stream by use of "(head of that the name of the main stream has changed by use of "(continuation of River)." River)" and 
As an added means of identification, each continuous- and partial-record station has been assigned a station number. The numbers have been assigned in the same downstream order used in this index. In assigning station numbers, gaps were left to allow for new stations that may be established; hence the numbers are not consecutive. The complete number for each station includes the part number, which is omitted in this index. The number given consists of only the essential digits of the complete number. For example, for a station with the complete number 03-0115.00, the station number shown in this index is 115 .

DRAINAGE AREA

The drainage area, in square miles, is the latest figure published or otherwise available at this time.

\author{
PERIODS OF RECORD
}

Under "Periods of record" are three columns. The first column, "Daily or monthly figures," shows the perlods of record for continuous-record gaging stations. The dates given are the calendar years in which records began or ended; breaks of less than a year are not shown. For example, If a record began in October 1923, ended in April 1932, began again in March 1933, and ended in September 1944, the period of record would be shown as 1923-44.

The second column, "Annual peaks," shows the period of record for crest-stage partial-record stations. The dates given are the water years for which the annual maximum discharge is available. The water year begins October 1 and ends September 30. In listing the water year, only one date is shown; for example, 1952 stands for the water year October 1, 1951, to September 30 , 1952.

The third column, "Low-flow measurements," shows the perlod of record for low-flow partialrecord stations. The dates given are the water years in which base-flow measurements were made at low-flow partial-record stations; breaks of less than two years are not shown. Prior to the 1958 water year, such measurements were published in tables headed "Miscellaneous discharge measurements" or "Discharge measurements at sites other than gaging stations." Many discharge measurements have been made at miscellaneous sites which are not listed in this index because the data collected were not sufficient to qualify the site as a low-flow partial-record station.

A date followed by only a dash shows that the station was continued in operation beyond December 31, 1963. A date followed by a period indicates discontinuance. A date followed by a semicolon indicates a break in the collection of records.

Periods of record for the same continuous-record station published by other agencles are listed only when they are for periods not published in reports of the Geological Survey. These periods are indicated by symbols and footnotes. Records collected by other agencles are included only for stations for which there is some record published in Geological Survey reports. 


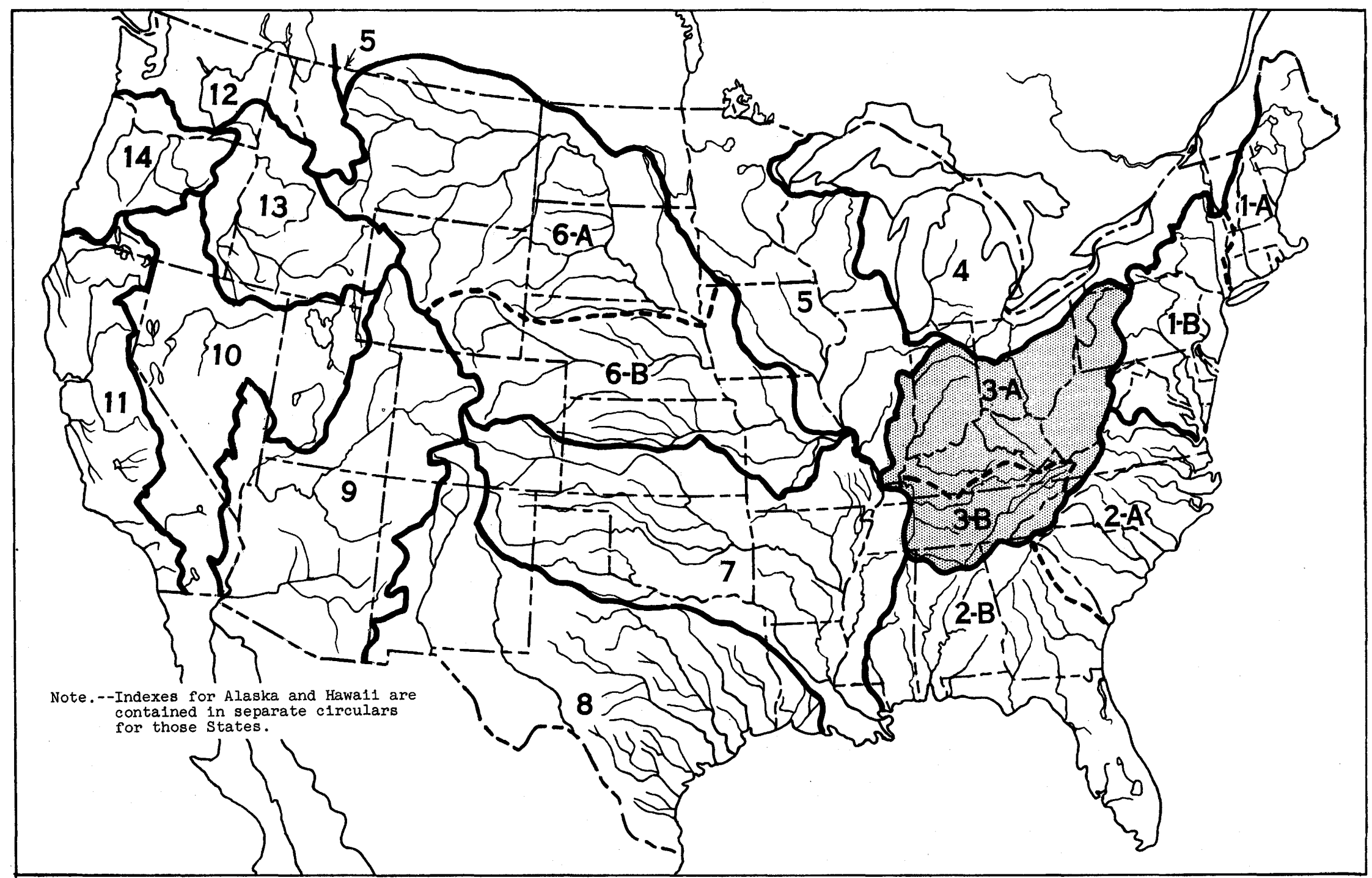

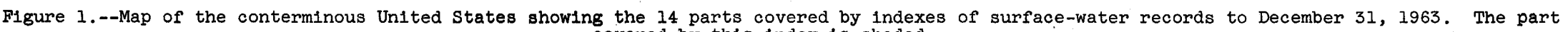




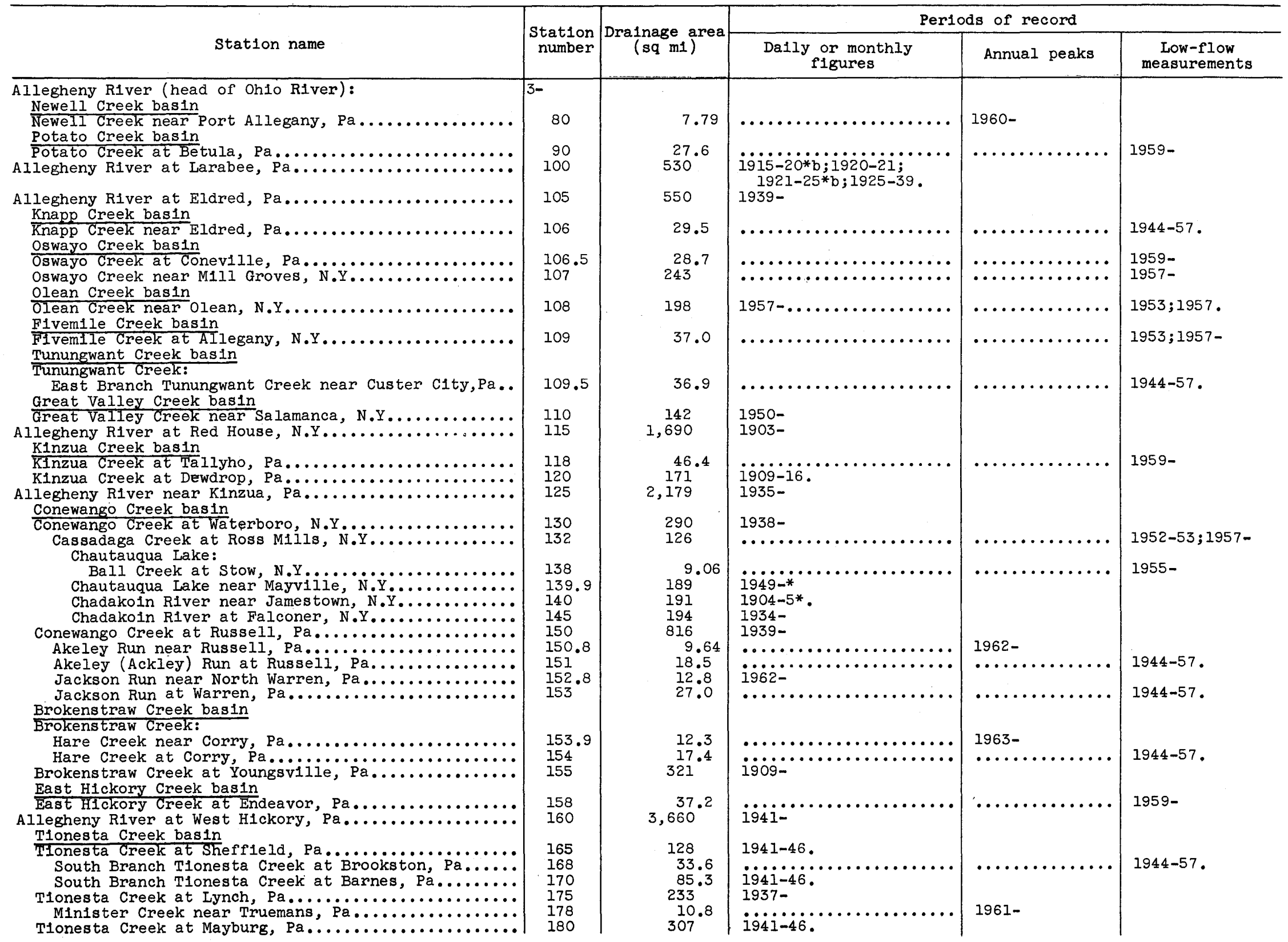


Tlonesta Creek at Butler Bridge (near Nebraska), Pa. 185

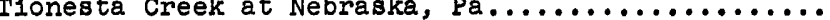
Tionesta Creek Reservolr at Tionesta Creek Dam, Pa. Tionesta Creek at Tionesta Creek Dam, $\mathrm{Pa} . \ldots \ldots \ldots \ldots$ Hemlock Creek basin

Hemlock Creek at President, $\mathrm{Pa} . . . \ldots \ldots \ldots \ldots \ldots \ldots$ 011 Creek basin

O11 Creek:

East Branch 011 Creek:

Fivemtle Creek near Buelis Corners, $\mathrm{Pa} . . . . . . . .$. Caldwell Creek:

West Branch Caldwell Creek near Grand Valley, $\mathrm{Pa}$. Caldwell Creek near Titusville, $\mathrm{Pa} . . . . . . . .$.

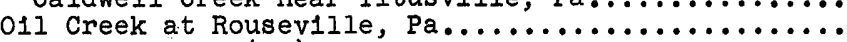
OII Creek near (at) Rousevilie, $\mathrm{Pa} . \ldots \ldots \ldots \ldots \ldots \ldots$ French Creek basin

French Creek at Carters Corners (Kimmeytown), Pa... Conneauttee Creek:

Little Conneauttee Creek near McKean, Pa........ Little Conneauttee Creek near Edinboro, $\mathrm{Pa} . . . .$. French Creek at Venango, $\mathrm{Pa} . \ldots \ldots \ldots \ldots \ldots \ldots \ldots \ldots$

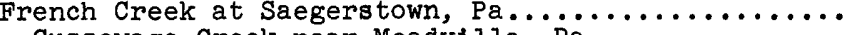

Cussewago Creek near Meadville, $\mathrm{Pa} . \ldots \ldots \ldots \ldots \ldots$

Van Horne Creek at Kerrtown, $\mathrm{Pa} . \ldots \ldots \ldots \ldots \ldots \ldots$

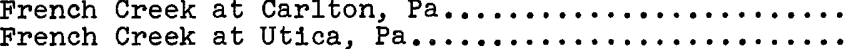
Middle Branch Sugar Creek.

West Branch Sugar Creek near Bradleytown, Pa....

Sugar Creek at Wyattville, $\mathrm{Pa} . \ldots \ldots \ldots \ldots \ldots \ldots \ldots$

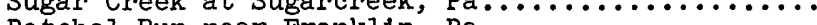

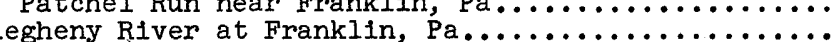

Allegheny River at Franklin

East Sandy Creek basin

East Sandy Creek

Sandy Creek basin

Sandy Creek near Sheakleyville, $\mathrm{Pa} \ldots \ldots \ldots \ldots \ldots \ldots$

Sandy Creek at Sandy Lake, $\mathrm{Pa} . \ldots \ldots \ldots \ldots \ldots \ldots$

Richey Run basin

Richey Run at Emlenton, $\mathrm{Pa} \ldots \ldots \ldots \ldots \ldots \ldots \ldots \ldots$

Clarion River basin

east Branch Clarion River:

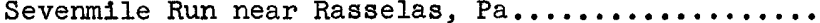
East Branch Clarion River Re'servoir at East Branch Clarion River Dam, Pa.

East Branch Clarion River at East Branch Clarion River Dam, $\mathrm{Pa}$.

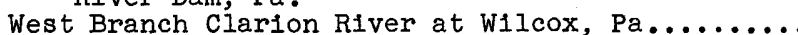
Clarion River at Johnsonburg, $\mathrm{Pa} \ldots \ldots \ldots \ldots \ldots \ldots \ldots$

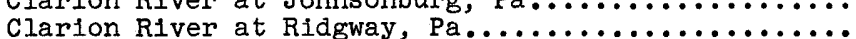

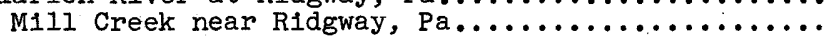

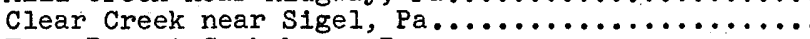

Toms Run at Cooksurg,

Clarion River at Cooksburg, $\mathrm{Pa} \ldots \ldots \ldots \ldots \ldots \ldots \ldots \ldots$

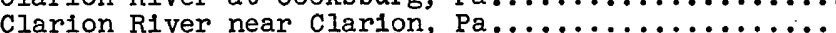

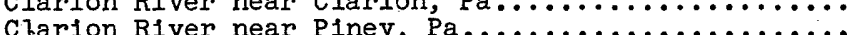

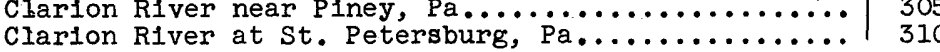

* Gage helghts, or gage helghts and discharge measurements only

a Approximately

$b$ In reports of Pennsylvania Department of Forests and Waters.

c In reports of U.S. Weather Bureau.

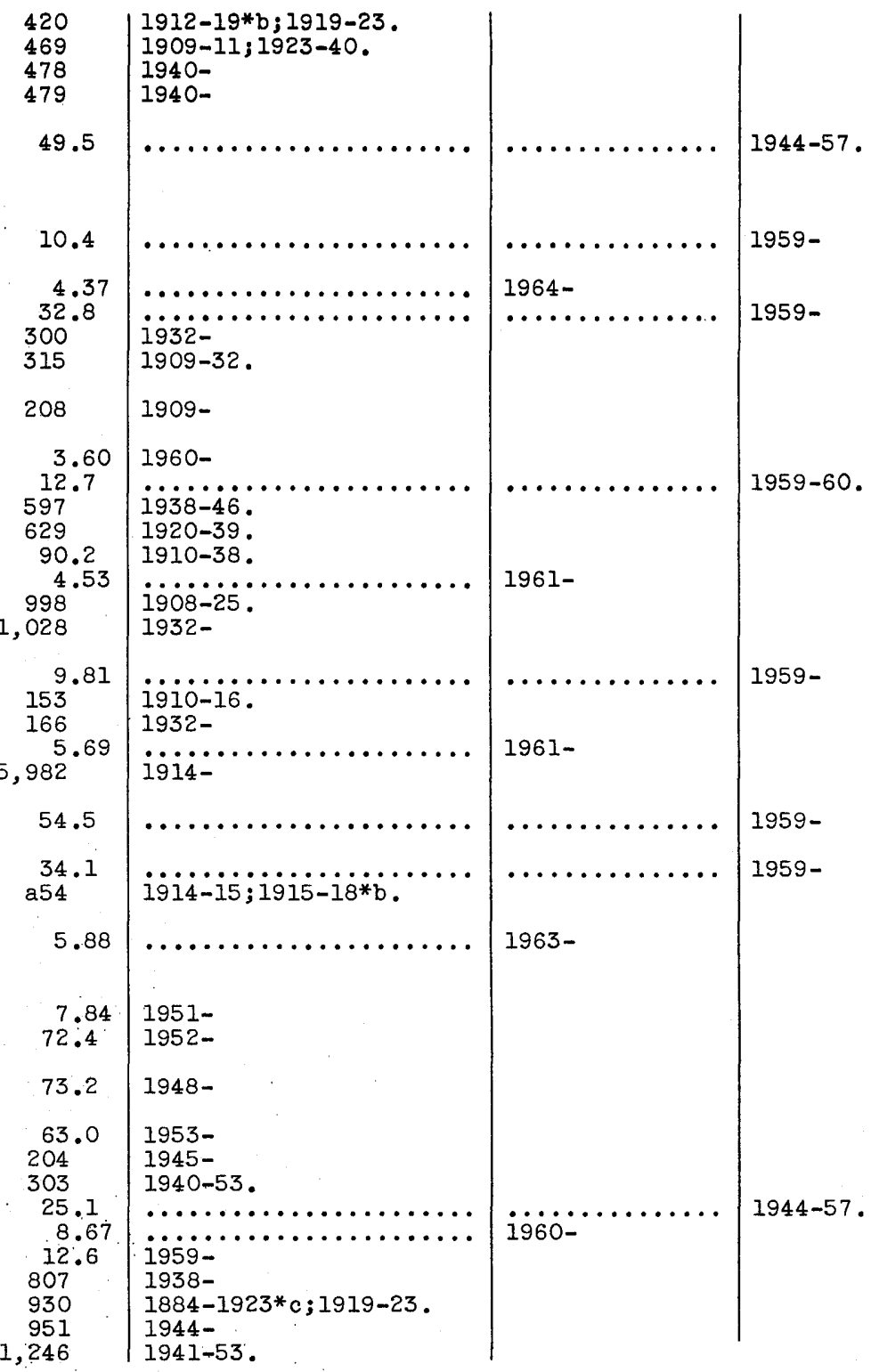




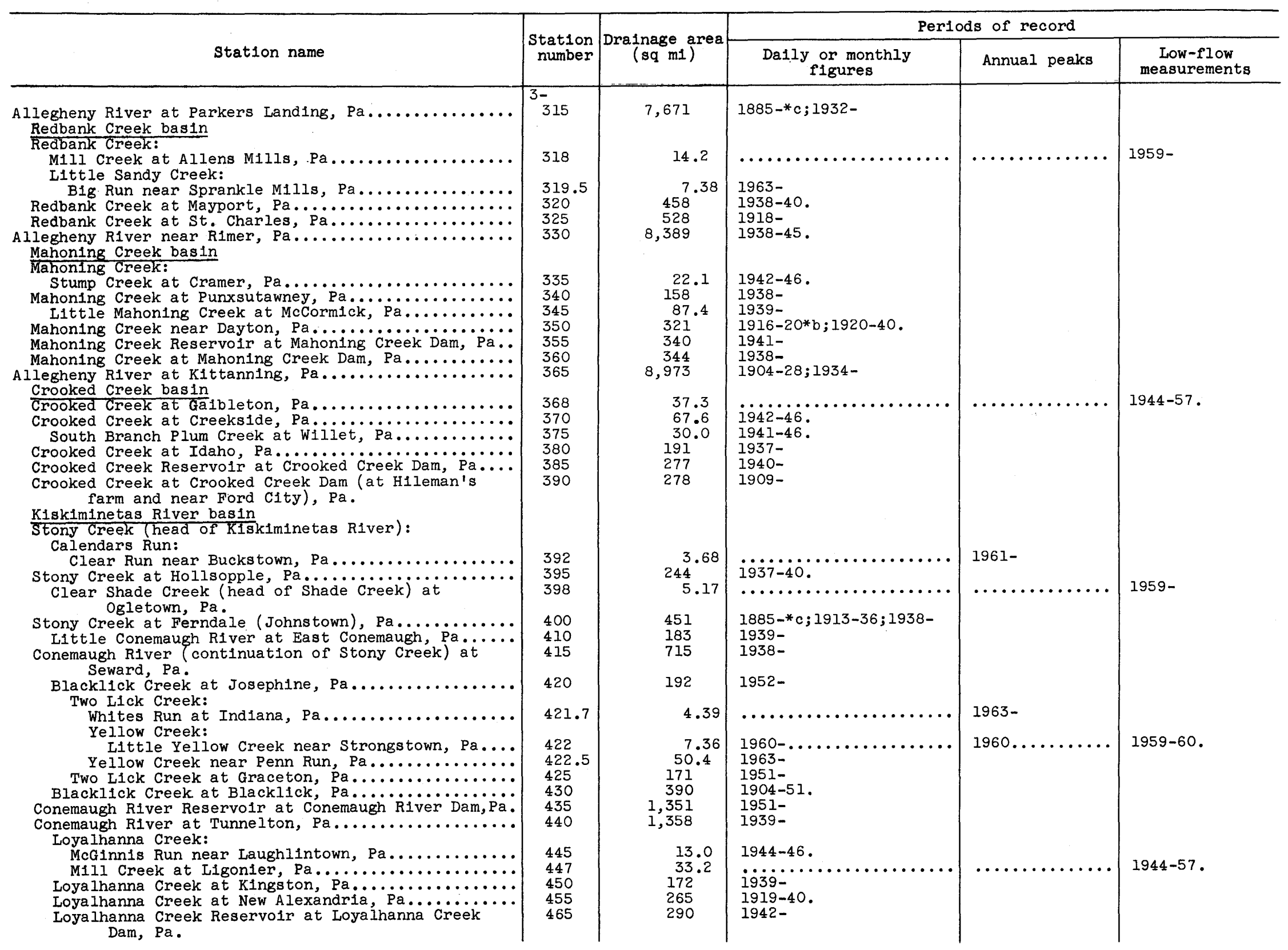


Loyalhanna Creek at Loyalhanna Creek Dam, $\mathrm{Pa} . . .$. Kiskiminetas River at Avonmore, $\mathrm{Pa} . \ldots \ldots \ldots \ldots \ldots \ldots$

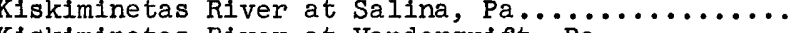
Kiskiminetas River at Vandergrift, $\mathrm{Pa} . . . \ldots \ldots \ldots \ldots$ Buffalo Creek basin

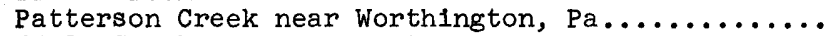

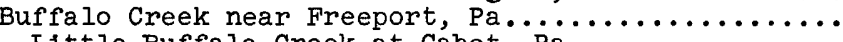

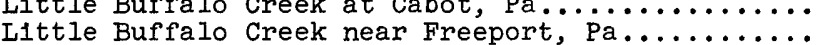

Allegheny River at Natrona, $\mathrm{Pa} . \ldots \ldots \ldots \ldots \ldots \ldots \ldots$

Chartiers Run basin

Chartiers Run near Leechburg, $\mathrm{Pa} \ldots \ldots \ldots \ldots \ldots \ldots$

Pine Creek basin

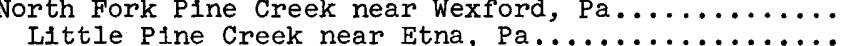

Tygart Valley River (head of Monongahela River) near Dalley, W. Va.

Tygart Valley River near Elkins, w. Va...........

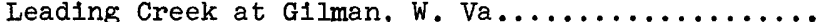
Tygart Valley River at Belington, W. Va..........

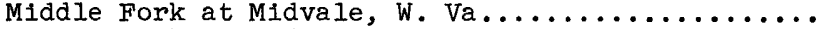
Middle Fork at Audra, W. Va................ Buckhannon River:

French Creek at Gould, W. Va

Sand Run near Buclchannon, $W$

Buckhannon River at HaIl W. Va....

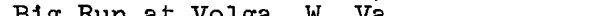

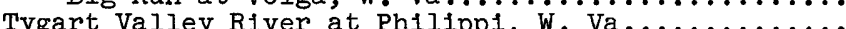

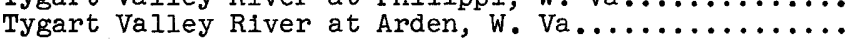

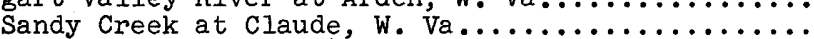

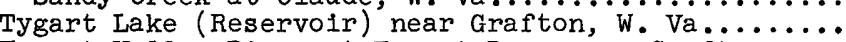

Tygart Valley River at Tygart Dam, near Grafton.....

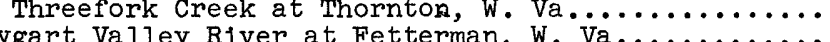

Tygart Valley River at Fetterman, W. Va..........

West Fork River

Skin Creek near Brownsville (Weston), W. Va.....

West Fork River at Brownsville, W. Va..........

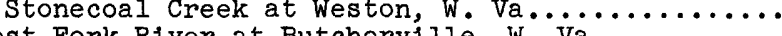

West Fork River at Butcherville, w. Va.........

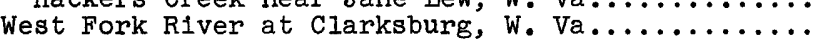

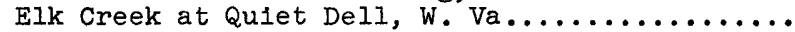

Tenmile Creek:

Salem Fork above Jacobs Run, at Salem, W. Va.. Jacobs Run:

Salem Fork subwatershed No. IIA (Varner Hollow Run) near Salem, W. Va.

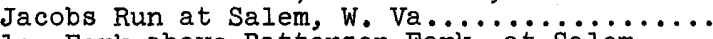

Salem Fork above Patterson Fork, at Salem.... Patterson Fork) near Salem, W. Va.

Patterson Fork at Salem, W. Va........... 602

* Gage heights, or gage heights and discharge measurements only. a Approximately.

$\mathrm{b}$ In reports of Pennsylvania Department of Forests and Waters.

c In reports of U.S. Weather Bureau.
$292 \quad 1939-$

1,723 1907-37.

$1,743 \quad 1904-5$.

1920-32*b; 1937 -

13.2

137

4.66

a.11, 410

1940

.

i938-

4.86

6.88

$\ddot{1} \dot{6} \dot{2}$

$187 \quad 1915-$

272 1944-

43.9

408

149

ïo-

1915-42.

1942-

40.1

277.5

6.19

916

945
76.7

1,184

1,184

90.9
, 304

1,366

25.7
102
36.0

36.0
181

54.3

84.6

1.67

1.67

.2

1.78
4.79

.79
.92

3.06

\section{$1946-$}

$1907-9 * ; 1915-$

$1941-45$.

1918-*c;1940-

i $\ddot{3} 38-$

i9o7-39.

1939-

$1946-60$
$1946-$

iिi்-

i923-

1943-

..

1954-62.

…..........

$i \dot{9} \dot{5} \dot{6}-\dot{6} \dot{2}$.
1955-61.

$1955-61$.
1959-

$1959-$

$1944-57$

$1959-$

1959-

1960-

1960-

$1960-$

$1960-$

$1961-$

........... 1960-

1960- 
Allegheny RIver--Continued

Monongahela River basin--Continued

Tygart Valley River--Continued

West Fork River--Continued

Tenmile Creek--Continued

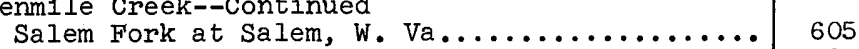

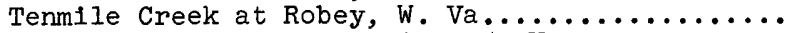

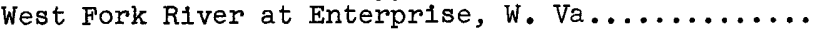
Monongahela River:

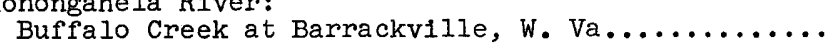

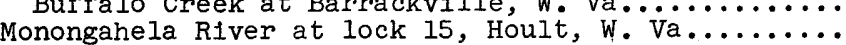

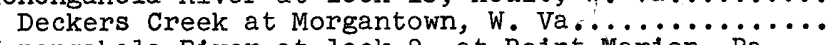

Monongahela River at lock 8, at Point Marion, $\mathrm{Pa} . .$.

Dry Fork (head of Cheat River):

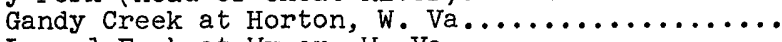

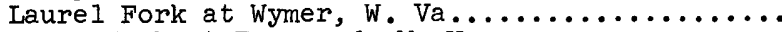

Glady Fork at Evenwood, W.

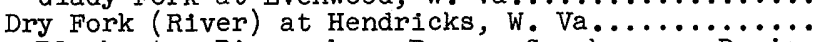

Blackwater R1ver above Beaver Creek, near Davis.

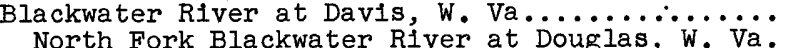

North Fork Blackwater River at Douglas, W. Va.

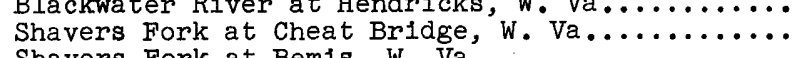

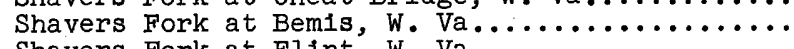

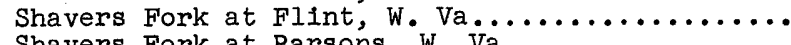

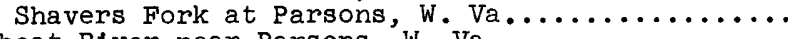

Cheat River near Parsons, W. Va.

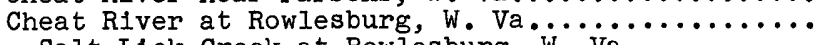

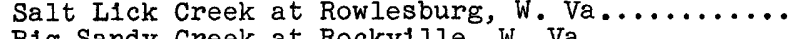

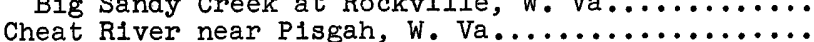

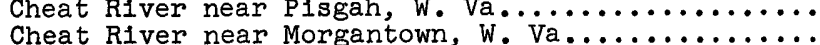

Dunkard Creek at Shannopin, $\mathrm{Pa} . \ldots \ldots \ldots \ldots \ldots \ldots$

Monongahela River at Greensboro, $\mathrm{Pa} \ldots \ldots \ldots \ldots \ldots \ldots$

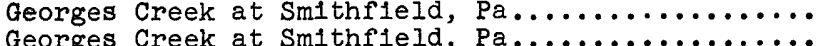

Georges Creek at Smithfield,

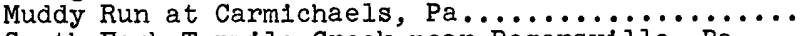

South Fork Tenmlle Creek near Rogersville, Pa.....

Browns Creek near Nineveh, $\mathrm{Pa} \ldots \ldots \ldots \ldots \ldots \ldots \ldots$

South Fork Tenmile Creek at Jefferson (Pöliäks $\mathrm{Mili)}, \mathrm{Pa}$

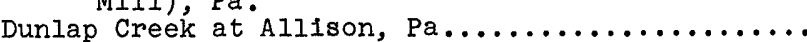
Redstone Creek:

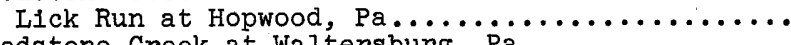

Redstone Creek at Waltersburg, $\mathrm{Pa} . . . \ldots \ldots \ldots \ldots . . . .$.

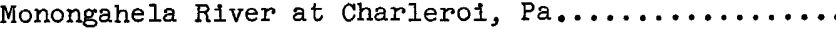

Youghiogheny River:

Snowy Creek:

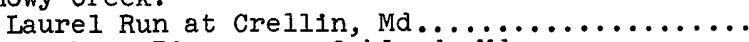

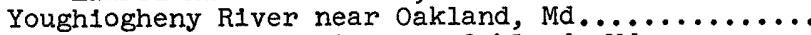

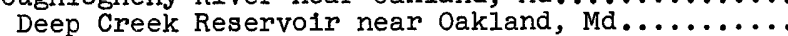

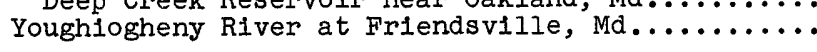

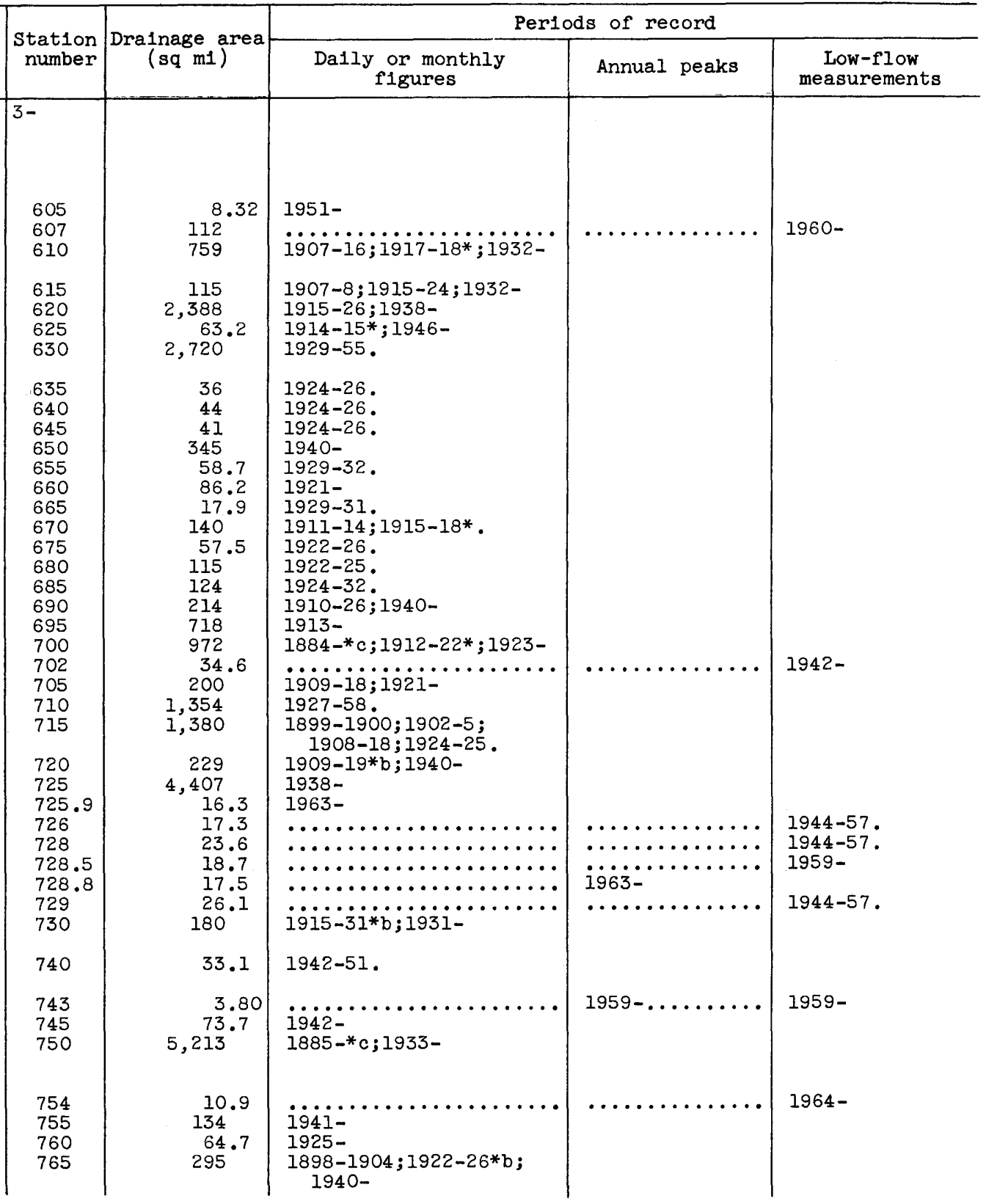


Youghiogheny River Reservoir at. Youghiogheny River Dam, $\mathrm{Pa}$

Youghlogheny River at Youghlogheny Dam

(Confluence), Pa.

Casselman River at Grantsville, Ma. .

Bic Piney Run near Salisbury, Pa...

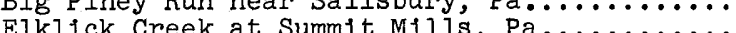

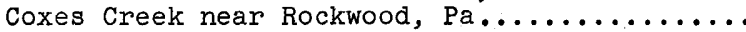
Middle Creek at Casselman, $\mathrm{Pa}$.

Cagselman River at Markleton, $\mathrm{Pa}$.

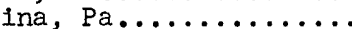

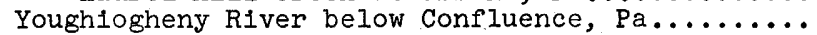
Drake Run near Confluence, $\mathrm{Pa} \ldots \ldots \ldots \ldots \ldots \ldots$

Youghlogheny River at Ohlopyle, $\mathrm{Pa} \ldots \ldots \ldots \ldots \ldots$ Indian Creek near Melcroft, $\mathrm{Pa} \ldots \ldots \ldots \ldots \ldots \ldots \ldots$

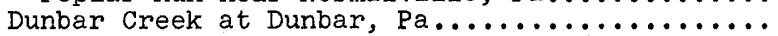

Youghlogheny River at Connelisvilie, $\mathrm{Pa} . . . \ldots \ldots$

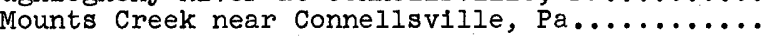
Jacobs Creek:

Green Lick Run at Green Lick Reservo1r, Pa.... Sewickley Creek:

Sewickley Creek tributary near Stanton, $\mathrm{Pa} . .$. Youghlogheny River at Sutersville, $\mathrm{Pa} . \ldots \ldots \ldots \ldots$ Gillesple Run near sutersville, $\mathrm{Pa} . . . . . . . .$. Turtle Creek:

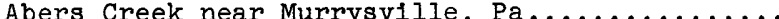

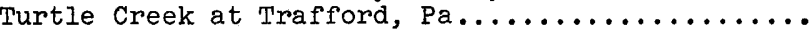

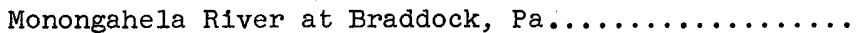
Chartiers Creek basin

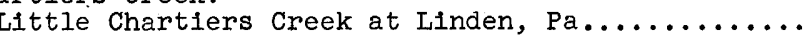

Chartiers Creek at Carnegle, $\mathrm{Pa} . \ldots \ldots \ldots \ldots \ldots \ldots \ldots$ Montour Run basin

Montour Run near Coraopolis, $\mathrm{Pa} \ldots \ldots \ldots \ldots \ldots \ldots \ldots$

Ohio River at Sewickley, Pa.

Big Sewickley Creek basin

B1g Sewickley Creek near Ambridge, $\mathrm{Pa} . \ldots \ldots \ldots \ldots \ldots$

Mahoning River (head of Beaver River) at Alliance, Ohio.

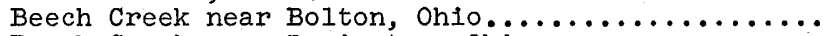

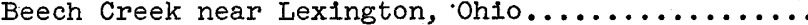

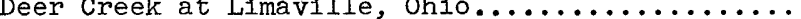

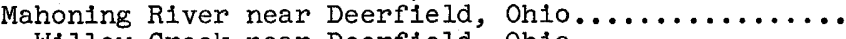

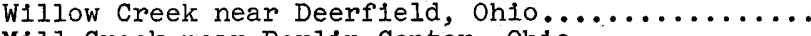

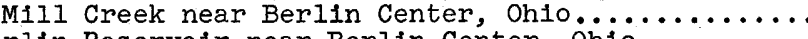

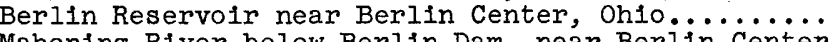

Mahoning River below Berlin Dam, near Berlin Center.

Milton Reservoir at Pricetown, ohio $\ldots \ldots \ldots \ldots \ldots \ldots \ldots$

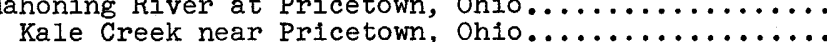

West Branch Mahoning River:

Hinkley Creek near Charlestown, Oh10.......... 921

Gage heights, or gage helghts and discharge measurements only.

a In reports of Pennsylvania Department of Forests and Waters.

c In reports of U.S. Weather Bureau.

\begin{tabular}{|c|c|c|c|c|}
\hline 770 & 434 & $1943-$ & & \\
\hline 775 & 436 & $1904-13 * ; 1904-22 * b ; 1939-$ & & \\
\hline $\begin{array}{l}780 \\
785\end{array}$ & $\begin{array}{l}62.5 \\
24.5\end{array}$ & $\begin{array}{l}1947- \\
1932-\end{array}$ & & \\
\hline 787 & 16.0 & & $\ldots \ldots \ldots \ldots \ldots$ & $1959-$ \\
\hline 788 & 38.1 & $\ldots \ldots \ldots \ldots \ldots \ldots \ldots \ldots$ & ............ & $1959-$ \\
\hline $\begin{array}{l}789 \\
790\end{array}$ & $382 \cdot 6$ & 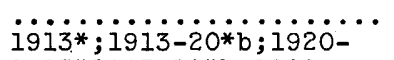 & $\cdots \cdots \cdots \cdots \cdots$ & $1944-57$ \\
\hline $\begin{array}{l}800 \\
810\end{array}$ & & $\begin{array}{l}1913 * 1913-18 * b ; 1918- \\
1940-\end{array}$ & & \\
\hline $\begin{array}{l}812 \\
815\end{array}$ & 1,062 & $\ddot{19} \ddot{2} 7-50 \ldots \ldots \ldots \ldots \ldots$ & $\ldots \ldots \ldots \ldots$ & $1959-$ \\
\hline $\begin{array}{l}821 \\
822\end{array}$ & $\begin{array}{c}32.6 \\
9.27\end{array}$ & $\ddot{i g} \ddot{6} \ddot{i}-\cdots \cdots \cdots \cdots \cdots \cdots$ & $\ldots \ldots \ldots \ldots$ & $1959-$ \\
\hline $\begin{array}{l}824 \\
825\end{array}$ & $1,326.8$ & $\ddot{i g} \ddot{s}-\cdots \cdots \cdots \cdots \cdots \cdots \cdots \cdots$ & $\ldots \ldots \ldots \ldots$ & $1944-57$ \\
\hline 826 & 18.9 & $\ldots \ldots \ldots \ldots \ldots \ldots$ & $\ldots \ldots \ldots \ldots$ & $1944-57$ \\
\hline 830 & 3.07 & 1941- & & \\
\hline $\begin{array}{l}832 \\
835\end{array}$ & $1,715.40$ & 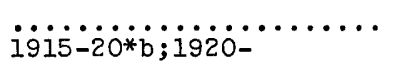 & $\cdots \cdots \cdots \cdots \cdots$ & 1959- \\
\hline 836 & 4.04 & $\ldots \ldots \ldots \ldots \ldots \ldots$ & $1959-$ & \\
\hline $\begin{array}{l}840 \\
845\end{array}$ & $\begin{array}{c}4.39 \\
55.9\end{array}$ & $\begin{array}{l}1948- \\
(1914 ; 1916-20) * b ; \\
1920-53 .\end{array}$ & & \\
\hline 850 & 7,337 & $1938-$ & & \\
\hline $\begin{array}{l}853 \\
855\end{array}$ & $\begin{array}{l}37.0 \\
257\end{array}$ & 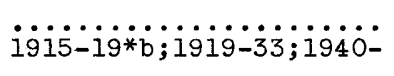 & $\cdots \ldots \ldots \ldots \ldots$ & $1944-57$ \\
\hline $\begin{array}{l}859 \\
860\end{array}$ & a $19,500^{29.4}$ & $i 933-\ldots \ldots \ldots \ldots \ldots \ldots$ & $\ldots \ldots \ldots \ldots$ & $1944-57$ \\
\hline 861 & 15.6 & $\ldots \ldots \ldots \ldots \ldots \ldots \ldots$ & $1963-$ & \\
\hline 865 & 87.9 & $1941-$ & & \\
\hline 870 & 18.8 & $1942-51$ & & \\
\hline $\begin{array}{l}875 \\
880\end{array}$ & $\begin{array}{l}32.4 \\
31.9\end{array}$ & $\begin{array}{l}1941-43 . \\
1941-51\end{array}$ & & \\
\hline 885 & $175^{\circ}$ & $1923-31$. & & \\
\hline $\begin{array}{l}890 \\
895\end{array}$ & $\begin{array}{l}11.7 \\
19.0\end{array}$ & $\begin{array}{l}1941-43 \\
1941-\end{array}$ & & \\
\hline $\begin{array}{l}900 \\
905\end{array}$ & $\begin{array}{l}249 \\
249\end{array}$ & $\begin{array}{l}1942- \\
1930-\end{array}$ & & \\
\hline 910 & 276 & $1923-$ & & \\
\hline $\begin{array}{l}915 \\
920\end{array}$ & 21.9 & $\begin{array}{l}1929- \\
1940-\end{array}$ & & \\
\hline 92 & 10.6 & $\ldots \ldots \ldots$ & $1947-$ & \\
\hline
\end{tabular}




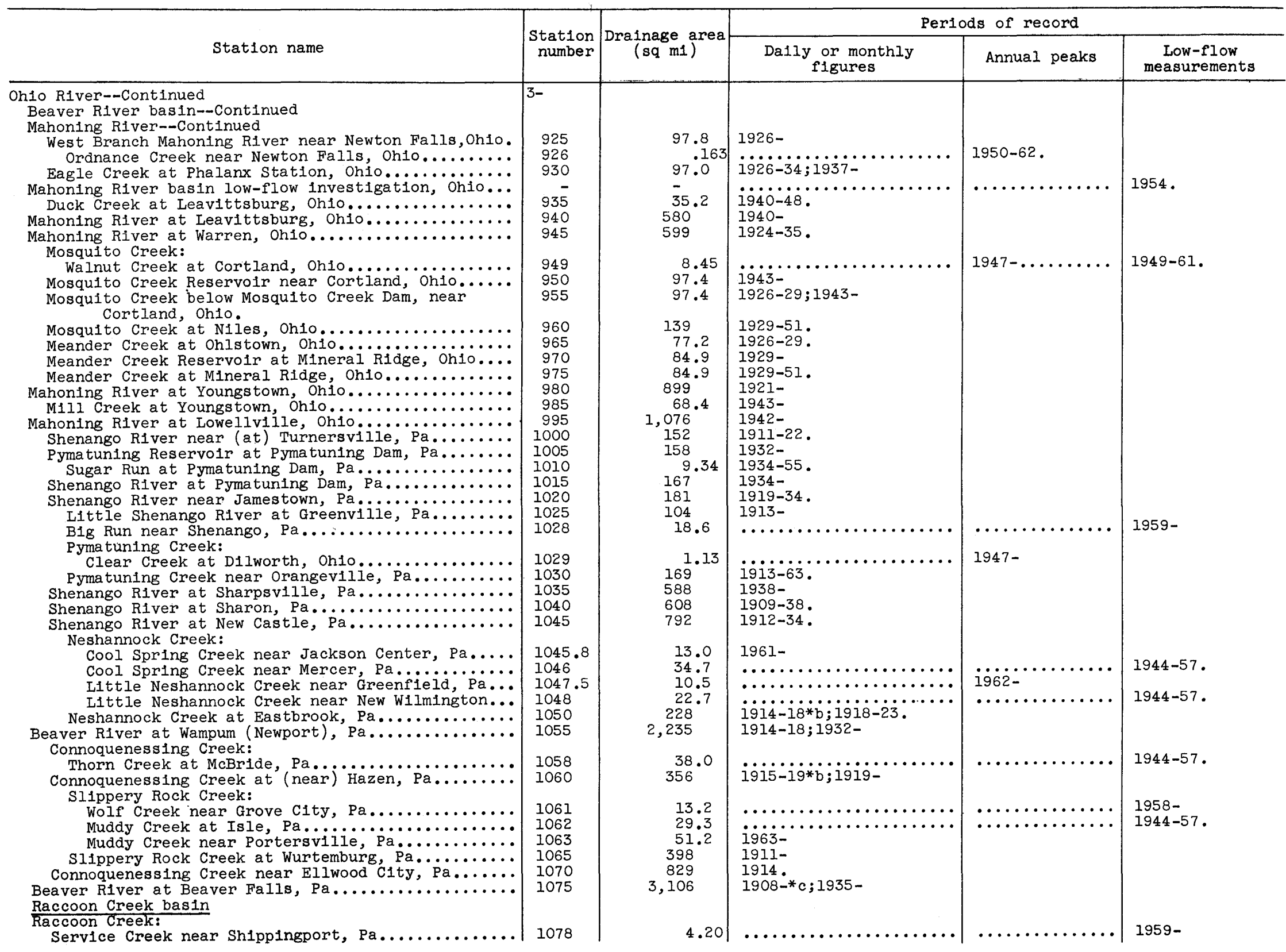


Raccoon Creek at Moffatts M111, Pa............. 1080

hio River at Montgomery Island Dam, $\mathrm{Pa} \ldots \ldots \ldots \ldots \ldots$ Little Beaver Creek basin

ladle Fork Iittle Beaver Creek:

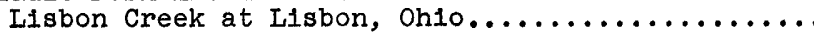
Little Beaver Creek near East Liverpool, Ohio....... Yellow Creek basin

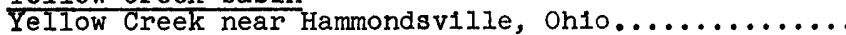

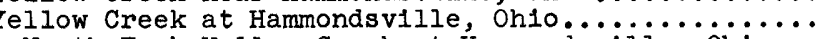
Torth Fork Yellow Creek at Hammondsville, Ohio Cross Creek basin

Cross Creek at (near) Mingo Junction, Ohio...........

\section{Buffalo Creek basin}

Buffalo Creek:

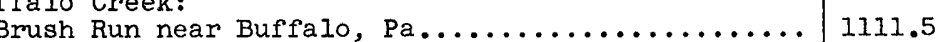

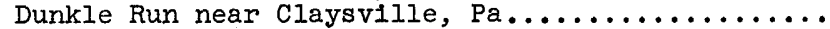

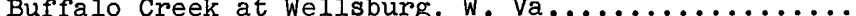
Short Creek basin

Short Creek near Dillonvale, Oh1o.

Wheeling Creek basin (Ohio)

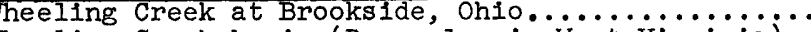

Wheeling Creek basin (Pennsylyania-West Virginia)

Wheeling Creek:

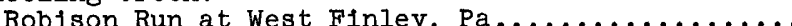

North Fork Dunkard Fork Wheeling Creek at Ryerson Station, $\mathrm{Pa}$

South Fork Dunkard Fork Wheeling Creek at Ryerson Station, $\mathrm{Pa}$

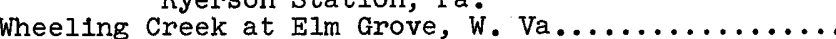

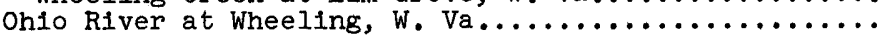

McMahon River basin

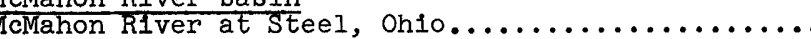

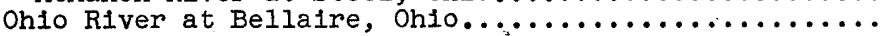

Captina Creek basin

Captina Creek at Armstrongs Mills, Ohio.......... Fish Creek basin

Fish Creek:

Pennsylvania Fork Fish Creek at Deep Valley, Pa...

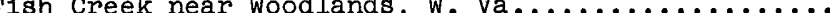
Sunfish Creek basin

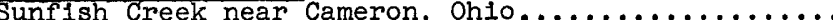

1shing Creek basin

righing Creek near New Martingville, W. Va.......... Middle Island Creek basin

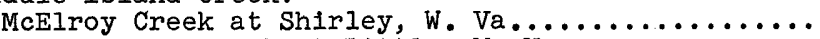

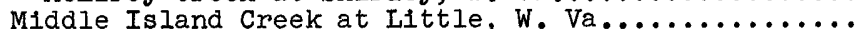

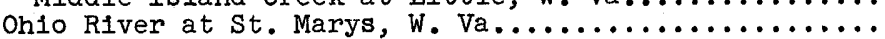
Little Muskingum River basin

Little Muskingum River at Bloomfleld, onlo.........

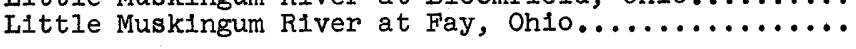

1080
1085
1090
1095
1100
1105
1106
1110

1111.5
1112
1113
1115
1115.5
1116
1117
1118
1120
1125
1130
1135
1140
1141
1142
1142.5
1143
1144
1145
1150
1154
1155
a22, 960

6.19

50

148

169

127

$1915-35$.

$1903 * \ldots \ldots \ldots \ldots$

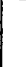

10.3

161

$1960-$

161

124

103

1941-

14.3
27.9

27.0

282

a24, 800

$a 82$
225,170

135

1882-** ; 1884 ; 1905-8;

$1936-37$.

$1903 *$.

1939-58.

135

1926-35; $1958-$

$199^{18.2}$

98.8

208

93.6

a.26, 850

210

259

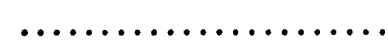

....................

$\ldots \ldots \ldots \ldots \ldots \ldots \ldots$

$\ldots \ldots \ldots \ldots \ldots \ldots \ldots$

igis-i6;igii $1904-* c ; 1938-$

$1958-$

1915-18; 1919-22*;
1959 ; $1962-$

1950-53; 1959;

1962 -

$1959-$

$1949-53 ; 1959-$

$1959 ; 1962-$

$1959-$

1944-57.

1944-57.

$1959-$

1960

$1959 ; 1962-$

1950; 1960-

1960-

* Gage heights, or gage heights and discharge measurements only.

a Approximately.

$b$ In reports of Pennsylvania Department of Forests and Waters.

c In reports of U.S. Weather Bureau. 


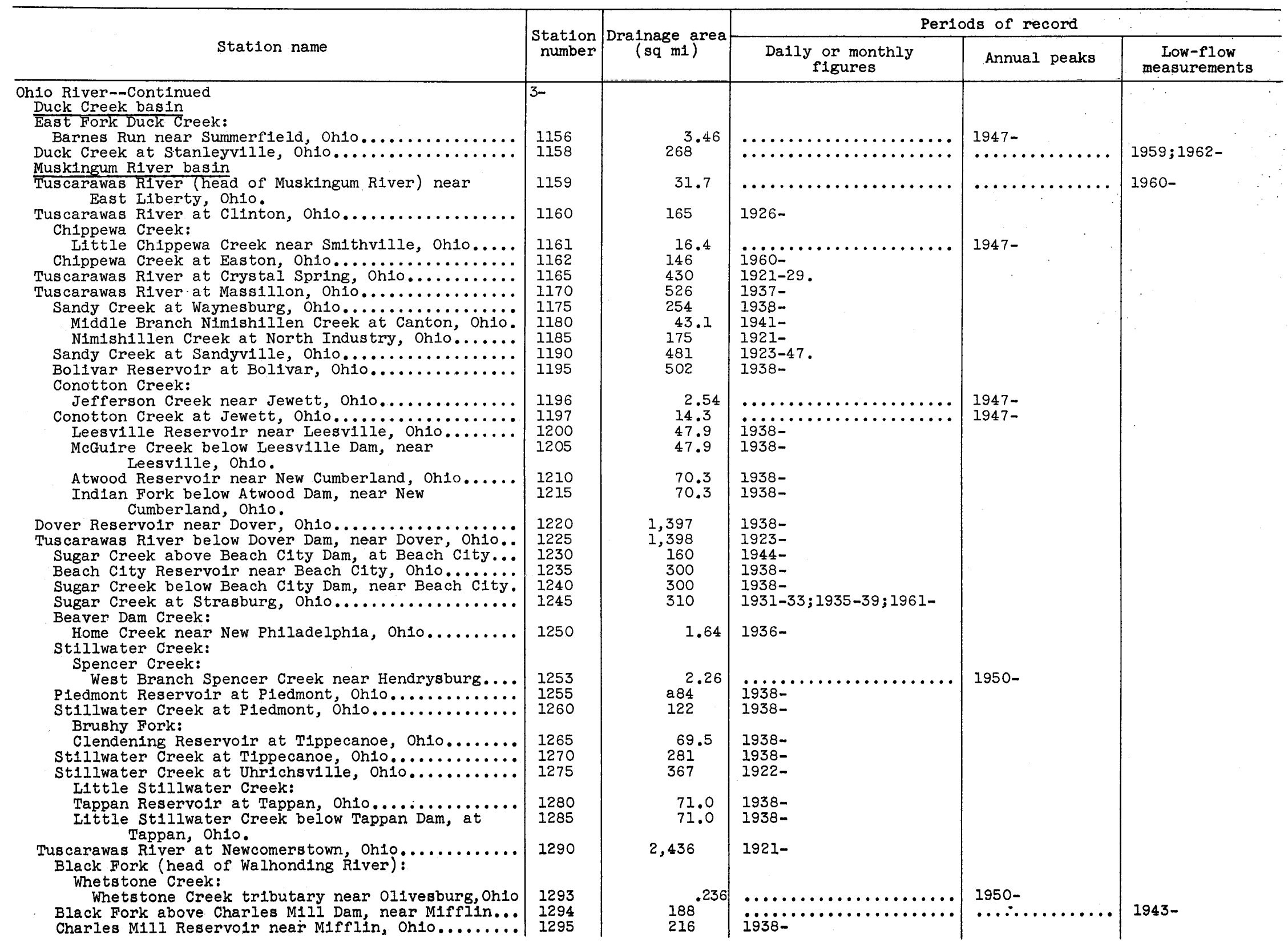


Black Fork below Charles Mill Dam, near Mifflin... Rocky Fork:

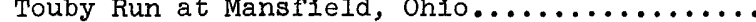

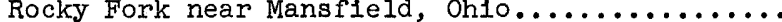

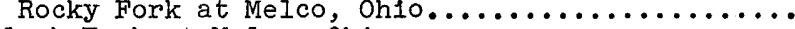

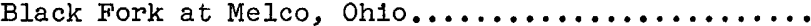

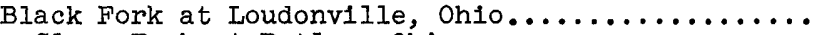

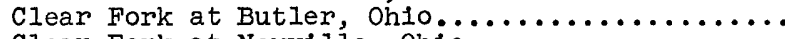
Clear Fork at Newville, Ohio ..................... Clear Fork below Pleasant Hill Dam, near Perrysville, Ohio.

Mohican River (continuation of Black Fork):

Jerome Fork (head of Lake Fork) at Jeromeville.. Mohicanville Reservoir near Mohicanville, Ohio.. Lake Fork below Mohicanville Dam, near Mohicanville, Ohio

$\ldots \ldots \ldots \ldots$

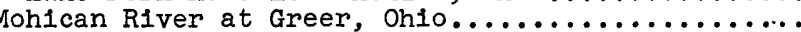
Kokosing River:

North Branch Kokosing River near Fredericktown

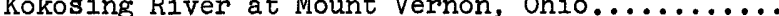

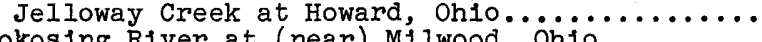

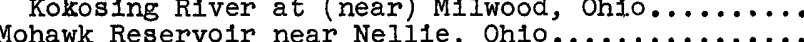
Walhonding (Mohican) River below Mohawk Dam, at

$$
\text { Nelile (Pomerene), Ohlo. }
$$

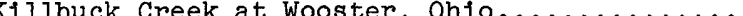

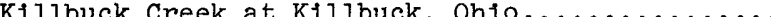

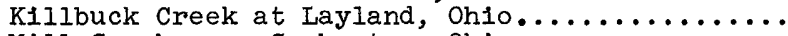

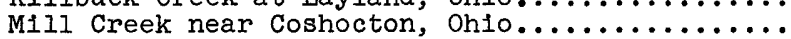

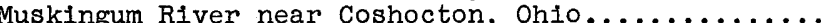
Buffalo Fork (head of W1lls Creek) at Pleasant City, ohio.

Buffe lo Creek at Pleasant City, Ohio........... Wills Creek:

Seneca Fork:

Senecaville Reservoir near Senecaville, Ohio.... Seneca Fork below Senecaville Dam, near Senecaville, Oh1o.

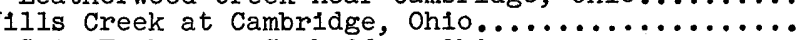

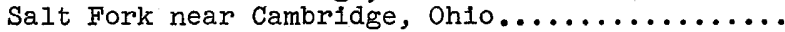

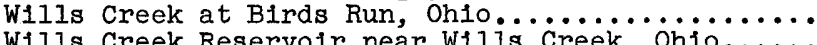

Wills Creek Reservoir near Wills Creek, Ohio......

Wills Creek below Wills Creek Dam, at Wills Creek.

Wakatomika Creek near Frazeysburg, Oh1o..........

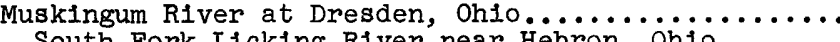

South Fork Licking River near Hebron,

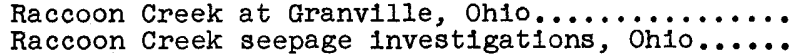

Raccoon Creek seepage inve

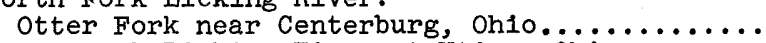

North Fork Licking River at Utica, Ohio.........

North Fork Licking River seepage investigations.

$\begin{array}{ll}\text { Llcking River near Newark, Ohio................. } & 1465 \\ \text { Licking River at Toboso (Pleasant Vailey), Ohio... } & 1470\end{array}$

* Gage helghts, or gage helghts and discharge measurements only.

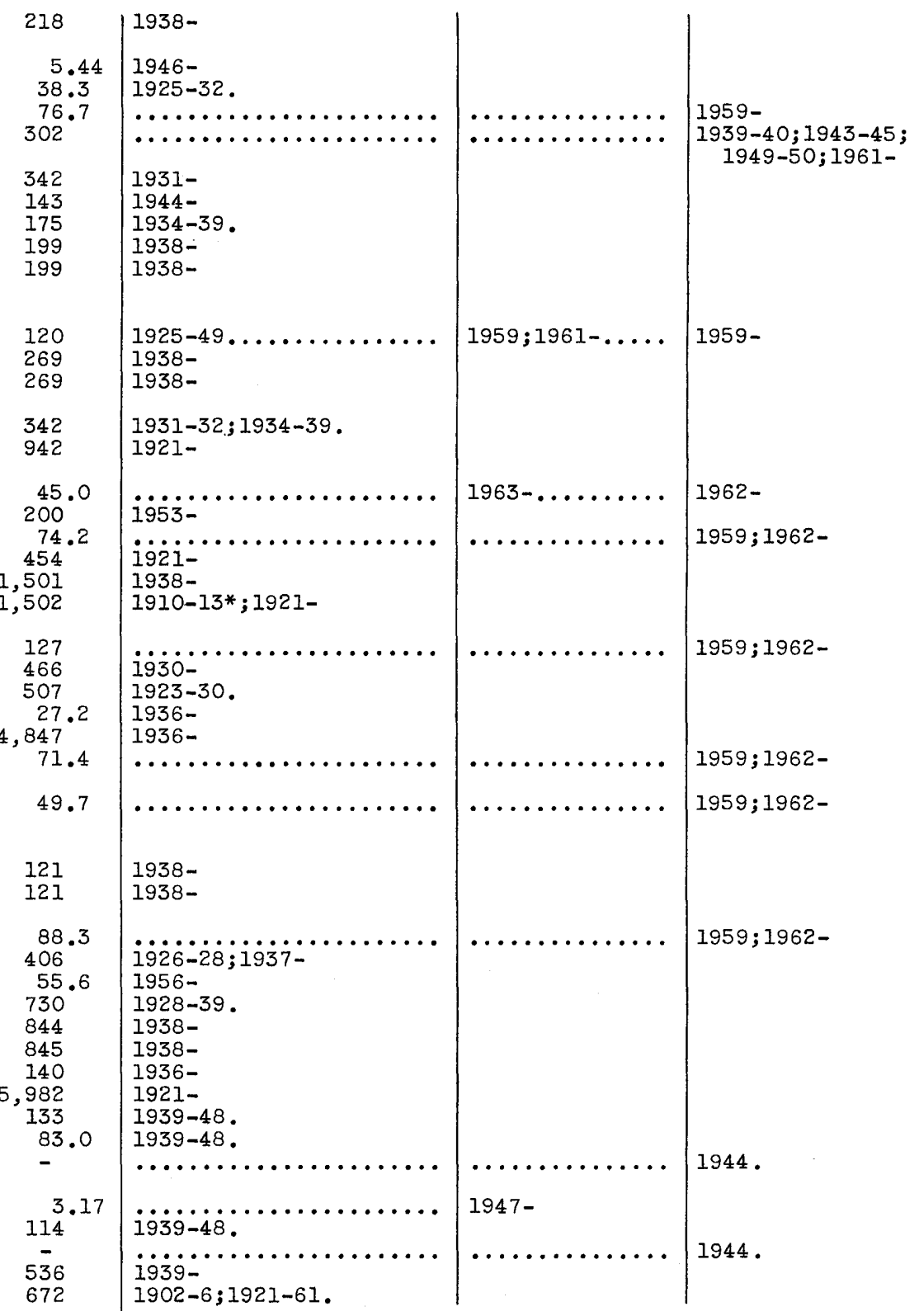




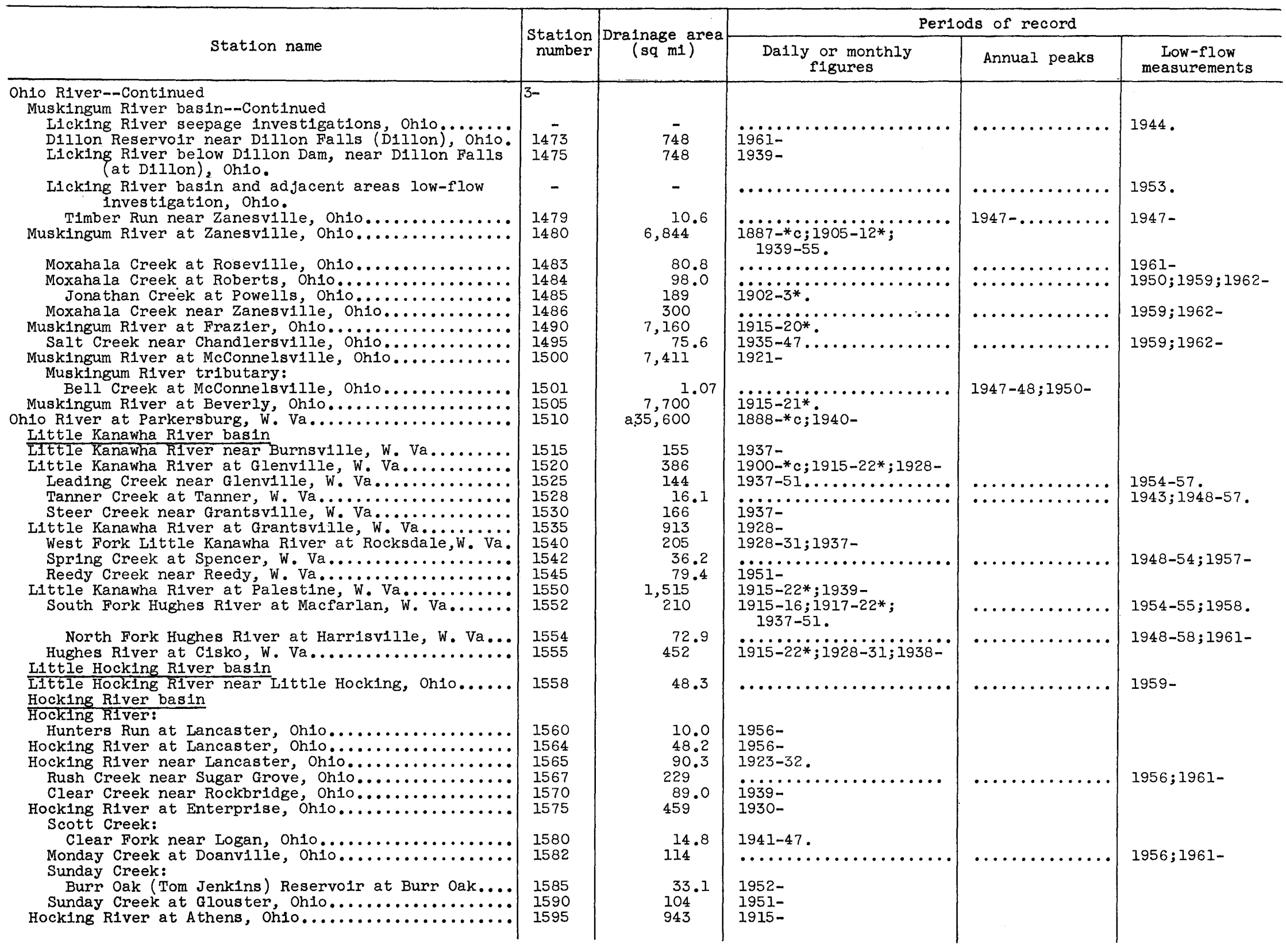


Federal Creek near Stewart, Oh1o Shade River basin

Shade River at Ke

Mill Creek basin

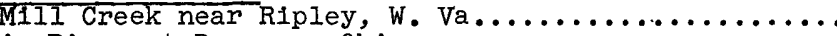
10 River at Pomeroy, Ohio.

Teading Creek near Middleport onio..

Kanawha River basin

Kanawha River basin River) near Blowing Rock, N.C.

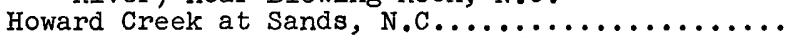

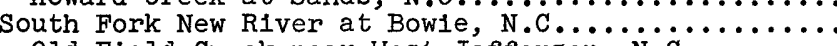

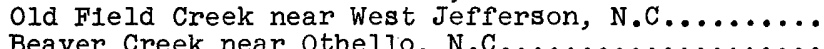

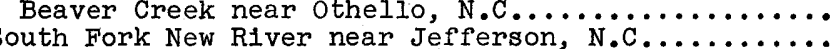

South Fork New River near Crumpler (at, near New River), N.C.

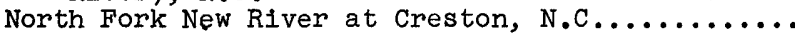

North Fork New River near Warrensville, N.C.......

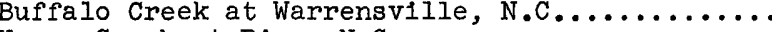

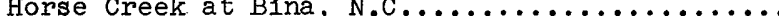

North Fork New River at (near) Crumpler

New River: (Weaversford), N.C.

Fox Creek:

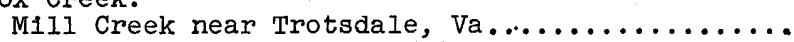

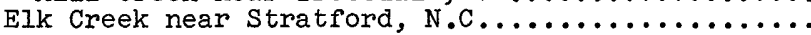

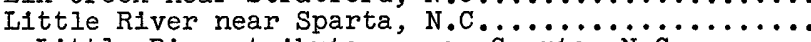

Little River tributary near Sparta, $N$. . . . . . . . .

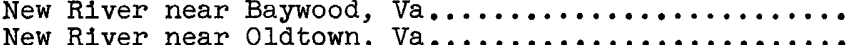

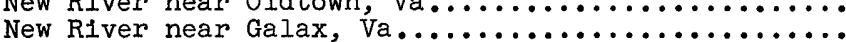

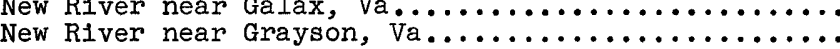

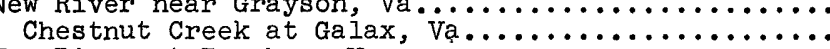

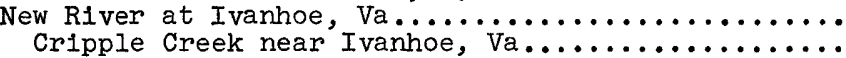

Reed Creek:

South Fork Reed Creek:

Neff-Litz Spring near Rural Retreat, Va......

Reed Creek at Grahams Forge, Va.............

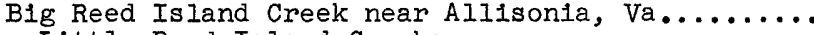

Little Reed Island Creek:

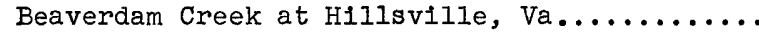

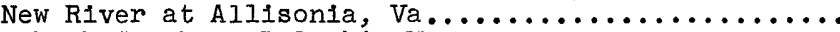

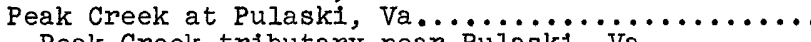

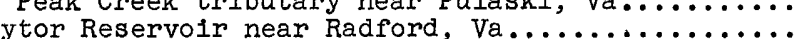

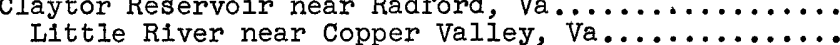

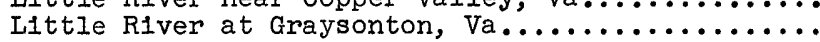

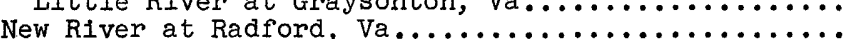

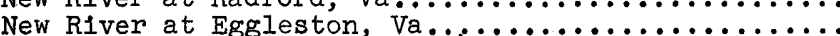

Walker Creek:

Helveys Mill Creek:

Helveys Mill Creek tributary at Point Pleasant

Wabash Spring near Poplar Hill, Va.................... 1720

* Gage heights, or gage heights and discharge measurements only.

a In reports of U.S. Weather Bureau.

d In reports of U.S. Weather Burge measurements only.

\begin{tabular}{|c|c|c|c|c|}
\hline 1595.2 & 136 & $\ldots \ldots \ldots \ldots \ldots \ldots \ldots$ & $\ldots \ldots \ldots \ldots$ & 1956 ; $1962-$ \\
\hline 1595.5 & 161 & $\ldots \ldots \ldots \ldots \ldots \ldots \ldots$ & $\ldots \ldots \ldots \ldots$ & $1956 ; 1962-$ \\
\hline $\begin{array}{l}1598 \\
1600\end{array}$ & $\begin{array}{r}132 \\
a 40,500\end{array}$ & 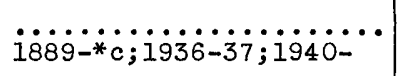 & $\ldots \ldots \ldots \ldots$ & $1944 ; 1947-54 ; 1960-$ \\
\hline 1600.5 & 118 & $\ldots \ldots \ldots \ldots \ldots \ldots \ldots$ & $\ldots \ldots \ldots \ldots$ & $1956 ; 1962-$ \\
\hline 1601.1 & 8.93 & $\ldots \ldots \ldots \ldots \ldots \ldots \ldots$ & ............. & $1950-56 ; 1960-$ \\
\hline $\begin{array}{l}1603 \cdot 1 \\
1605 \\
1606 \cdot 1 \\
1608 \cdot 7 \\
1610 \\
1615\end{array}$ & $\begin{array}{l}10.2 \\
129 \\
22.4 \\
217 \\
207 \\
325\end{array}$ & 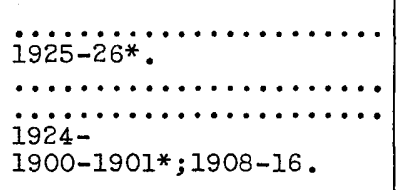 & $\begin{array}{l}\ldots \ldots \ldots \ldots \ldots \\
1955- \\
\cdots \ldots \ldots \ldots \ldots\end{array}$ & $\begin{array}{l}1955-62 \\
1963-\end{array}$ \\
\hline $\begin{array}{l}1618.8 \\
1620 \\
1621.1 \\
1622.2 \\
1625\end{array}$ & $\begin{array}{l}63.1 \\
113 \\
223 \\
259 \\
277\end{array}$ & 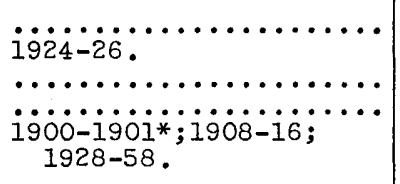 & $\begin{array}{l}\cdots \ldots \ldots \ldots \cdots \\
1955- \\
\cdots \cdots \cdots \cdots \cdots\end{array}$ & $\begin{array}{l}1949-56 ; 1960-62 . \\
1955-56 ; 1959-\end{array}$ \\
\hline $\begin{array}{l}1628 \\
1628.4 \\
1628.6 \\
1628.8 \\
1630 \\
1635 \\
1640 \\
1645 \\
1650 \\
1655 \\
1660\end{array}$ & $\begin{array}{c}5.32 \\
\text { a12. } \\
19.3 \\
a 3.5 \\
a 1,000 \\
\overline{1}, 131 \\
1,160 \\
a 39 \\
1,340 \\
148\end{array}$ & 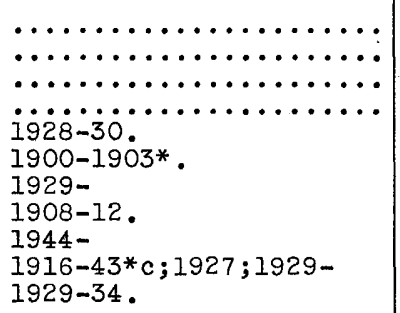 & $\begin{array}{l}1951- \\
\cdots \ldots \ldots \ldots \ldots \ldots \\
1955-\end{array}$ & $\begin{array}{l}1955-56 ; 1962- \\
1951-56 .\end{array}$ \\
\hline $\begin{array}{l}1665 \\
1670 \\
1675\end{array}$ & $\begin{array}{l}- \\
247 \\
278\end{array}$ & $\begin{array}{l}(1928-29 ; 1947-56) d \\
1908-16 ; 1927- \\
1908-16 ; 1939-\end{array}$ & & \\
\hline $\begin{array}{l}1677 \\
1680 \\
1685 \\
1686 \\
1690 \\
1695 \\
1700 \\
1710 \\
1715\end{array}$ & $\begin{array}{c}4.13 \\
2,202 \\
60.9 \\
2,382^{.61} \\
239 \\
300 \\
2,748 \\
2,941\end{array}$ & $\begin{array}{l}1932 \ldots \ldots \ldots \ldots \ldots \ldots \\
1927-33 ; 1951-57 \ldots \ldots \ldots \ldots \\
1939 \ldots \ldots \ldots \ldots \ldots \\
1908-16 . \\
1928- \\
1895-* c ; 1907-15 ; 1939- \\
1914-\end{array}$ & $\begin{array}{l}1962- \\
1958-61 \\
1949 ; 1951-\end{array}$ & \\
\hline $\begin{array}{l}1718 \\
1720\end{array}$ & -.38 & $\ddot{1} \ddot{9} \dot{9}-5 i \dot{1} . \cdots \cdots \cdots \cdots \cdots$ & $1957-$ & \\
\hline
\end{tabular}




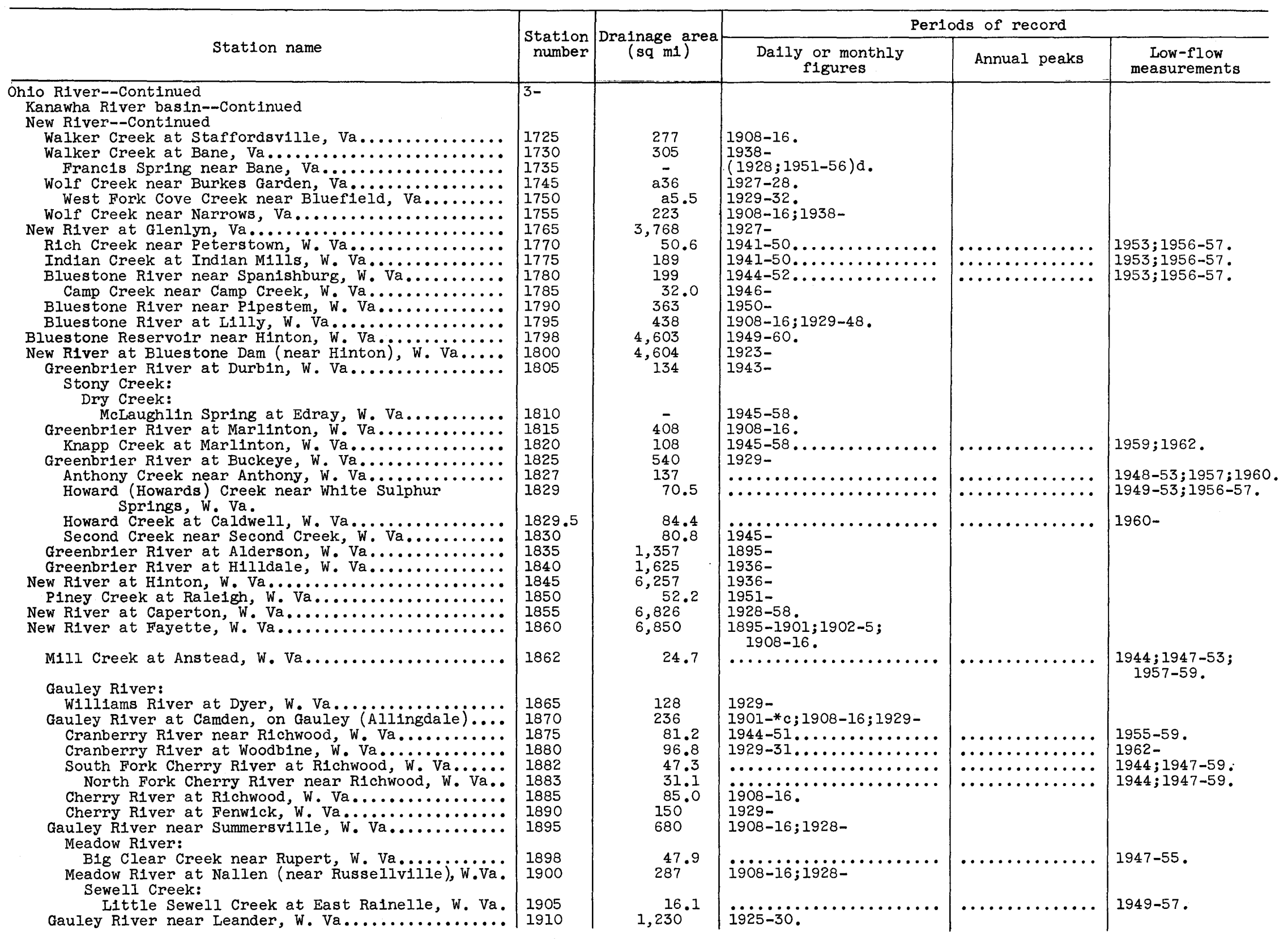


Peters Creek near Lockwood, W. Va............ 1915 Gauley River above Belva, W. Va

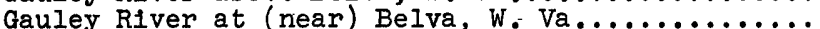
Kanawha River at Kanawha Falls (at lock 2 , Montgomery), W. Va.

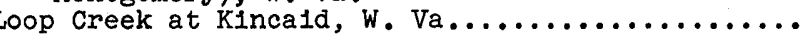

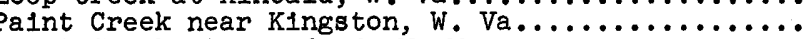
Paint Creek at Hansford, W. Va.

(n) Elk Rlver near Webster Springs, $W$. Va..........

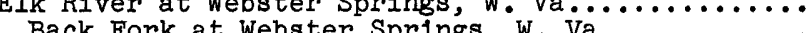
Back Fork at Webster Springs, W. Va $3 \ldots \ldots \ldots \ldots . . .$. Elk River below Back Fork at Webster Springs, Wa. Elk River below Webster Springs, W. Va.........

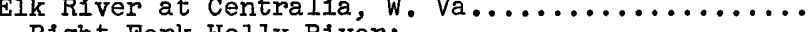
Right Fork Holly River:

Left Fork Holly River at Hacker Valley, W. Va.

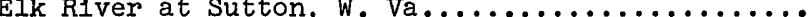

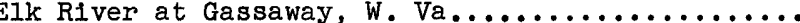
Birch River near Birch River, W. Va $\ldots \cdots \cdots$ Birch River at Herold, W. Va...

Elk River near Frametown, W. Va.............

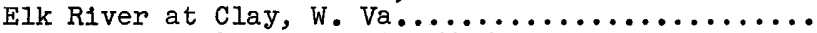

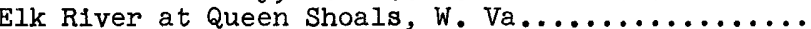
Big Sandy Creek near Amma, W. Va.............

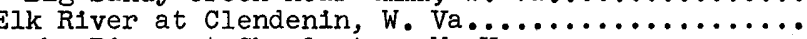

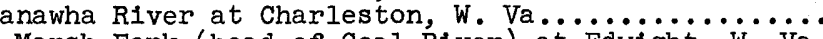
Marsh Fork (head of Coal River) at Edwight, W. Va. Clear Fork at Ameagle, W. Va.................. Spruce Fork (head of Little Coal River) at Clothier, W. Va.

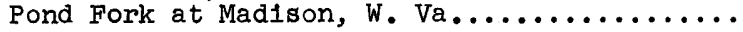

Little Coal River at Danville (Madison), W. Va.. Little Coal River at McCorkle, W. Va..........

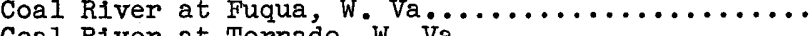

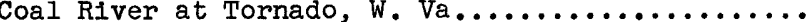

Pocatalico (Pocataligo) River at Sissonville,W.Va. Hurricane Creek near Hurricane, W. Va...........

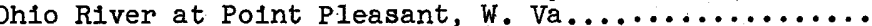
Raccoon Creek basin

Sandy Run near Lake Hope, Ohio.

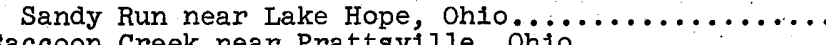

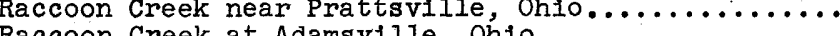

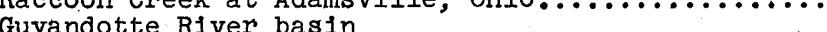
Guyandotte River basin

Cuyandote

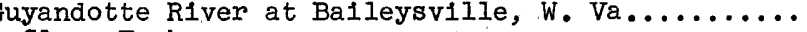

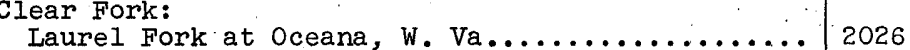

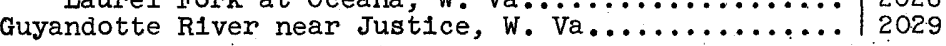

* Gage helghts, or gage heights and discharge measurements only. a Approximately.

c In reports of U.S. Weather Bureau.

d Discharge measurements only.

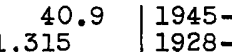

$1,402 \quad 1908-16$

\begin{tabular}{l|l}
1,402 \\
8,367
\end{tabular}

16.6

126.9

124

169

171

70.4
242

268

281

29.3

543

578

48.3
124

752

994

1,145

84.4

1,290

10,419
128

128
38.4

393

51.6

138

270
375

375

849
861

831

238

a52, $760^{17}$

2018

2020

6

only

\begin{tabular}{|c|c|}
\hline $\begin{array}{l}4.99 \\
200 \\
587\end{array}$ & $\begin{array}{l}1957- \\
i \ddot{9} i \overrightarrow{5}-\ddot{3} \dot{5} ; \ddot{i} \dot{3} \ddot{8}-\ldots \ldots \ldots \ldots\end{array}$ \\
\hline $\begin{array}{l}261 \\
354\end{array}$ & 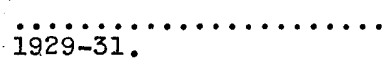 \\
\hline $\begin{aligned} 56.7 \\
512\end{aligned}$ & $\ddot{B} \ddot{B}$ \\
\hline
\end{tabular}

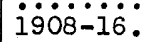

$1 \ddot{9} 2 \dot{9}-3 \dot{4}$.

$1959-$

1943-63.

i93̈-

1908-16.

$1945-48$.

$1958-$

$1915-* c ; 1958-$

1928-

i9os-í.

$1939-$

...............

$\ddot{1908-i 6} ; \dot{19} \ddot{3} \dot{-}$

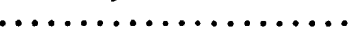

1930-

$1915-17$; 1918-22*.

$1911-16$.

$1908-11 ; 1912 * ; 1928-31$.

$1908-16$; 1930-31; $1937-$

.............................
$1947-57$

1948-57 ; 1947 ; 1957 -

$1947 ; 1953$

…................... 194

1947-59

$1948-59$.

$1930-31 ; 1939-41$

1960-61.

$1960-$

$1947-53 ; 1960-61$

$1947-53 ; 1957$.

$1947-53$.

$1944 ; 1947-53$;

1960-61.

1944 ; 1947-53;

1957-61.

1960-

1951-53;1959-

$1948-55$; 1960 -

1948-55. 


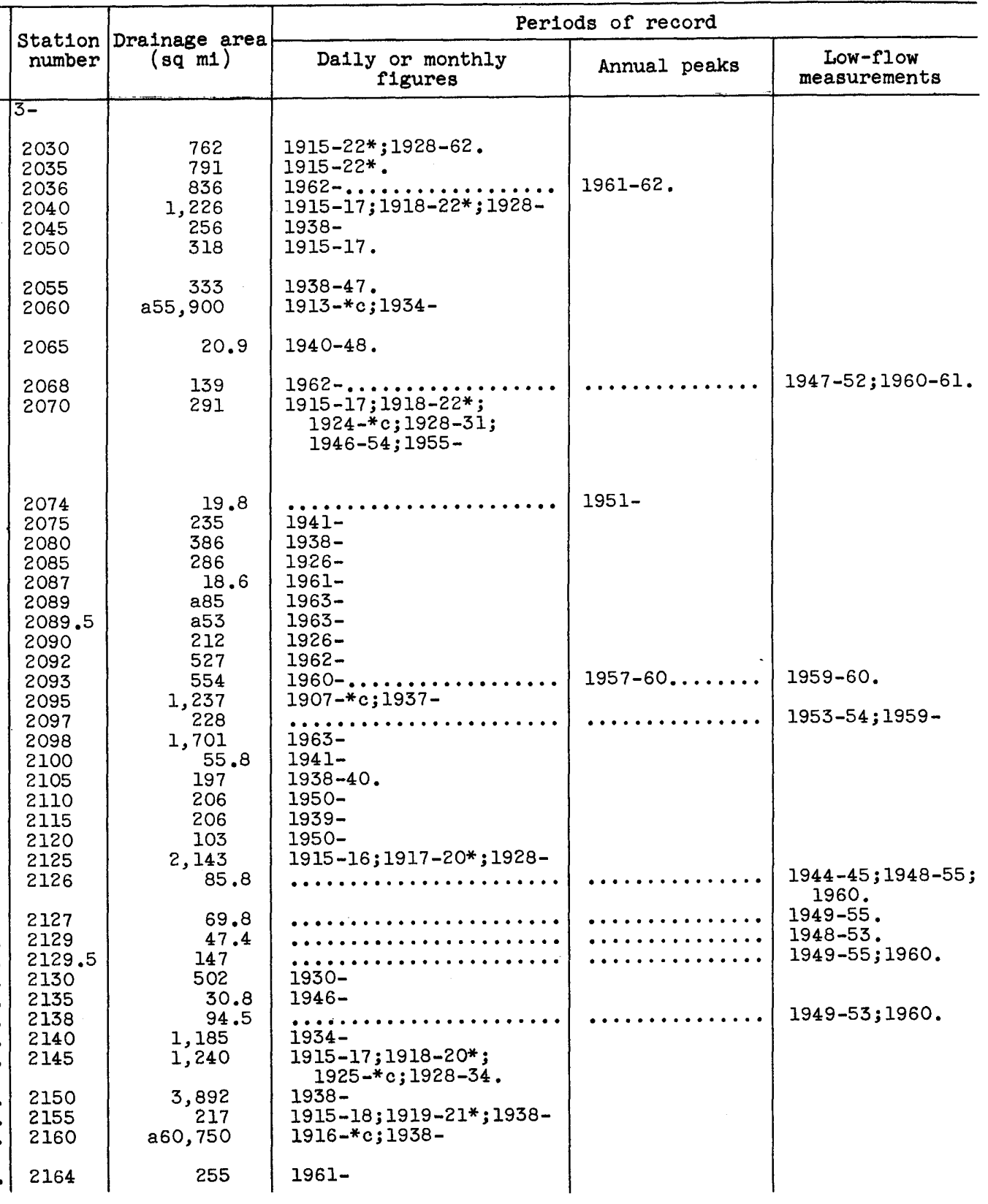

Oh10 River--Continued

Guyandotte River basin--Continued

Guyandotte (Guyandot) River at Man, W. Va.

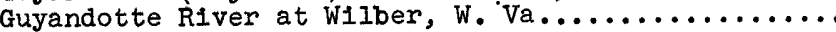

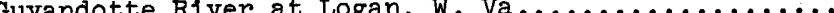

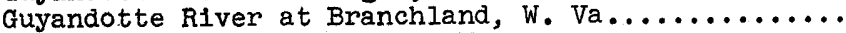

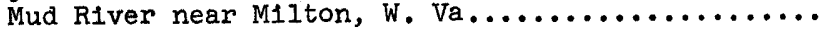

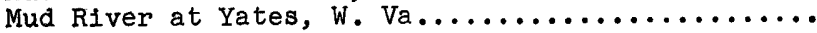

Symmes Creek bas in

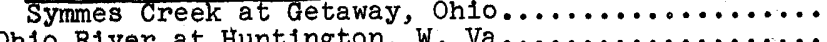

10 RIver at Huntington, W. Va.

Fourpole Creek basin

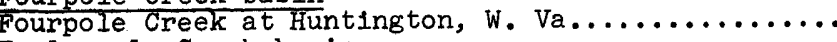

Twelvepole Creek basin

East Fork Twelvepole Creek near East Lynn, W. Va....

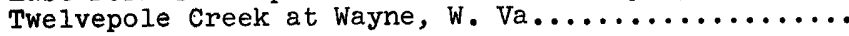

Big Sandy River basin

Levisa Fork (head of Big Sandy River):

Prater Creek at Vansant, Va $\ldots \ldots \ldots \ldots \ldots \ldots \ldots$

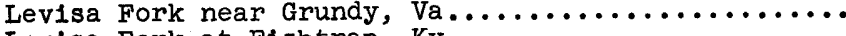

Levisa Fork at Fishtrap, Ky $\ldots \ldots \ldots \ldots \ldots \ldots \ldots \ldots$

Russell Fork at Haysi, va $\ldots$ Pound, Va. $\ldots \ldots \ldots \ldots_{\ldots} \ldots \ldots \ldots$

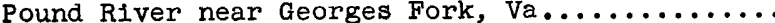

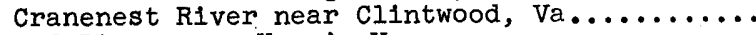

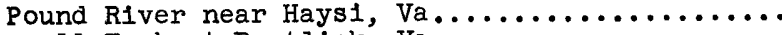

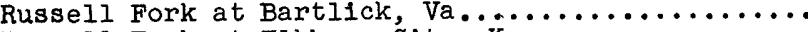

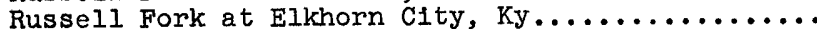

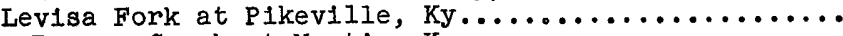

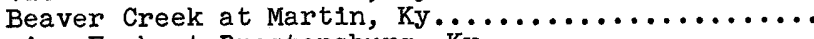

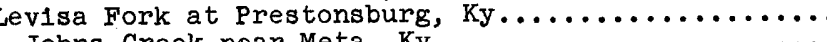

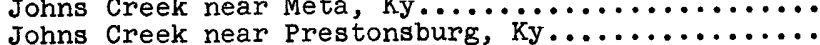

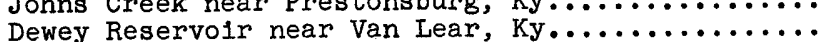

Dewey Reservolr near Van Lear, Ky $\ldots \ldots \ldots \ldots \ldots \ldots$

Johns Creek near Van Lear, Ky...

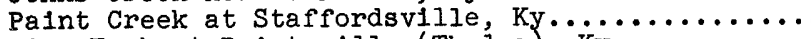

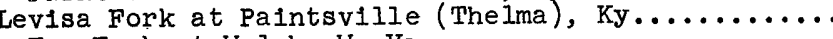

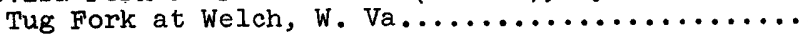

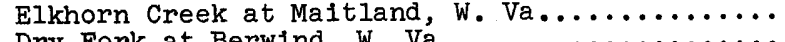
Dry Fork at Berwind, w. Va $\ldots \ldots \ldots \ldots \ldots \ldots \ldots \ldots \ldots$

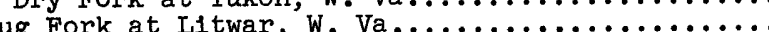

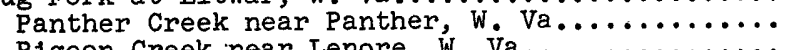

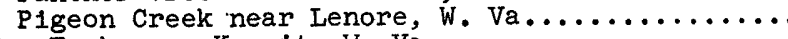

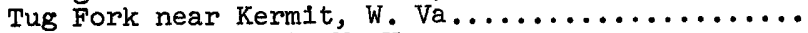

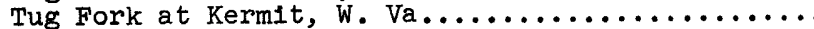

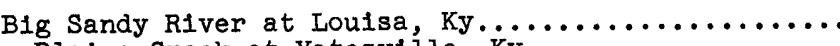

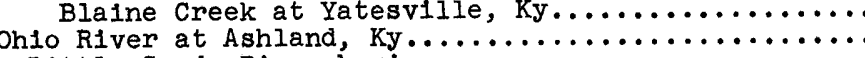
Little Sandy River basin

Little Sandy River at Leon, Ky 


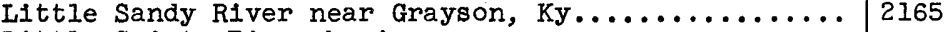

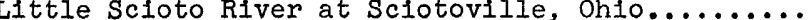
Tygarts Creek basin

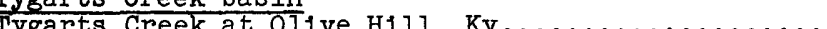

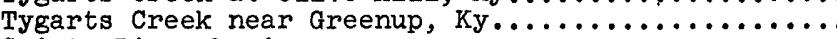
Scloto River basin

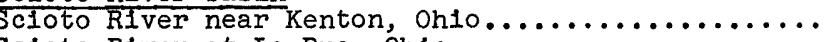

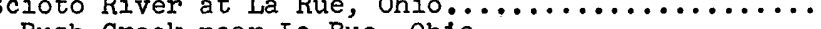

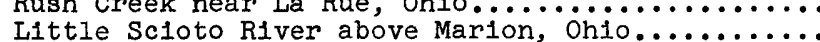
Little Scloto River above Marion, Ohio.............. near Marion (near Marion), Ohio.

Little Sc1oto Rion

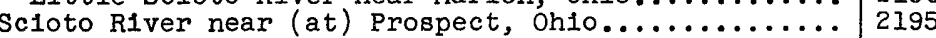

Prairie Run:

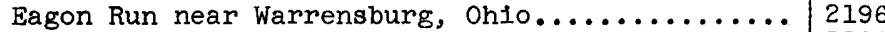

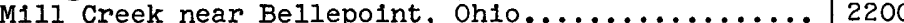

o'Shaughnessy Reservoir near Dublin, Oh10........... 2205

Scloto R1ver bèlow O'Shaughnessy Dam, near Dublin near Dublin), Ohio.

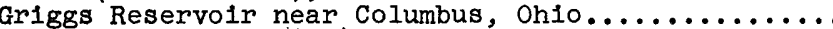

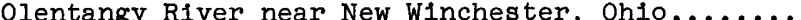

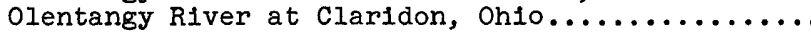

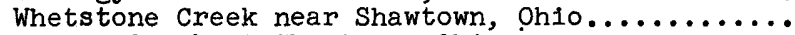

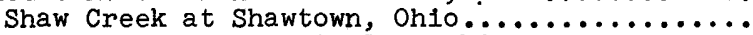

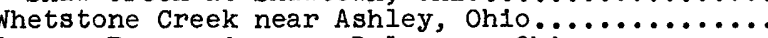

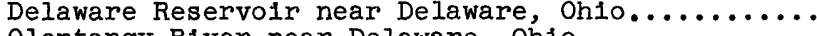

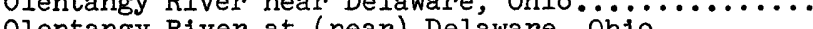

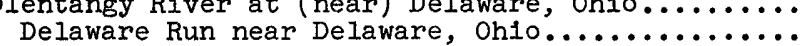

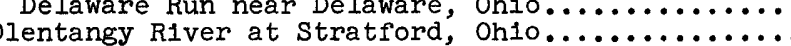

olentangy River at stratiord, ohio $\ldots \ldots \ldots \ldots \ldots \ldots \ldots$

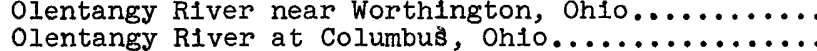

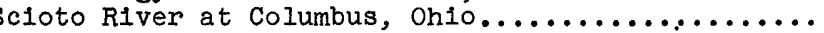

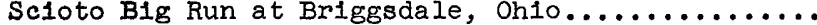

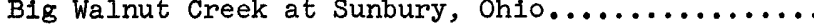

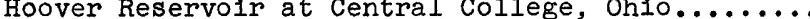

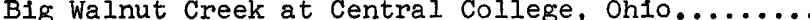

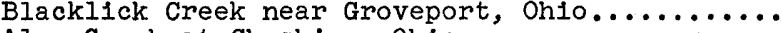

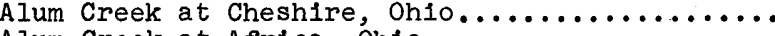

Alum Creek at Africa, Ohio.

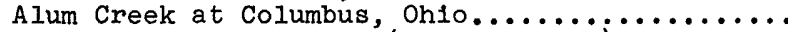

Big Walnut Creek at Rees (Rees Station), Ohio.....

Big Walnut Creek basin low-flow investigations....

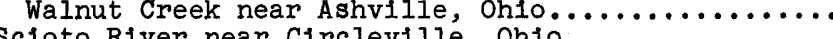

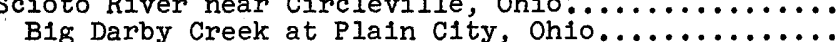

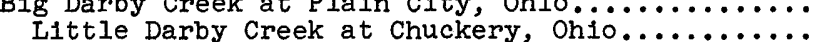

Big Darby (Darby) Creek at Darbyville, Ohio......

Hominy Creek at Circleville, Ohio.......... 2306

Deer Creek at W1lliamsport, Ohio.............. 2310

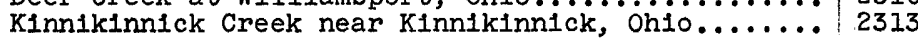

* Gage helghts, or gage heights and discharge measurements only.

a Approximately. $U . S$. Weather Bureau.
1938-

59.6

242

129

88.5
72.4

85.8

93.3

567

178

.123

1915-*c;1925-32;1939-

979
980

$1950-62$

1942-

980 1921-

\begin{tabular}{l|l}
1,044 & $1921-$ \\
1,067 & $1898-19$
\end{tabular} $1904-6$.

$1946-49$.

$1946-$

61.8

25.4

98.7

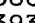

1946-55.

$1946-55$

$1954-$

1923-34; $1938-$

5.84

445

$1910-* c ; 1921-23$

497

1,629

11.0

1898-1900; $1903-5$.

1920

1946-58..............

ï호-

$1938-$

57.2

105

122

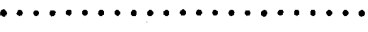

$963-\cdots \cdots \cdots \cdots \cdots \cdots$

$1923-35 ; 1938-$

1921-35;1938-

285
638

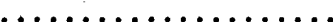

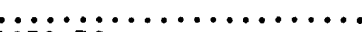

$1938-56$.

151.

534.4

534

і9азі-з5; 1938 -

5.66

333

36.

$1926-35 ; 1938-56 ; 1962-\ldots$

$1959-62$

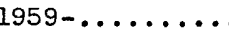

1959-61.

1956 ; $1961-$

1950;1959-

1956;1961-62.

.

1962 .

1948 .

1954 ; 1961-

$1950 ; 1961-$

$1950 ; 1962-$

$1947 ; 1952 ; 1956$ 1962 -

1954 ; 1958 ; $1961-$ 


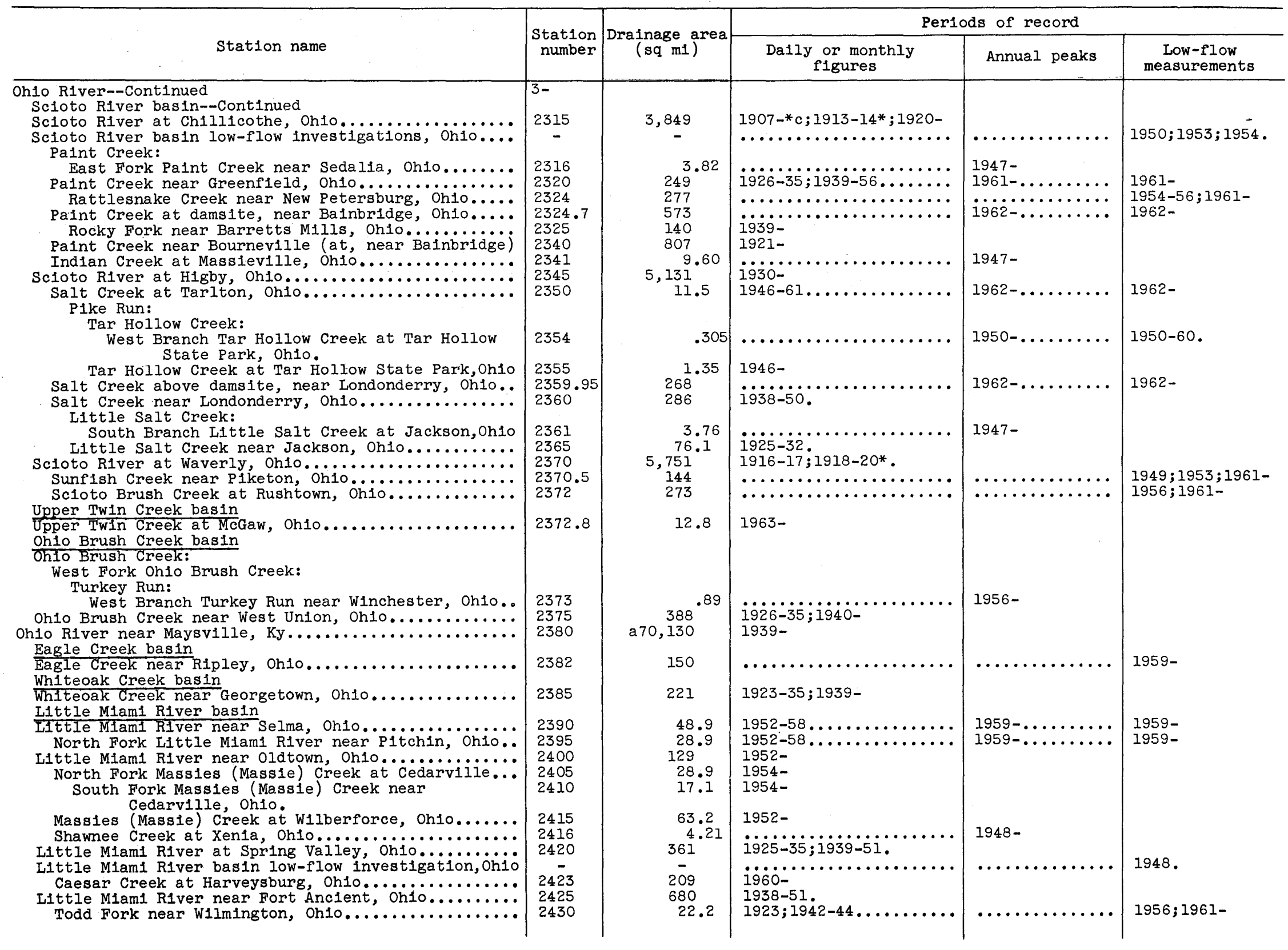


Cowan Creek at Clinton County Air Force Base....

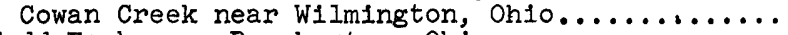
Todd Fork near Roachester, Oh10 $\ldots \ldots \ldots \ldots \ldots \ldots$

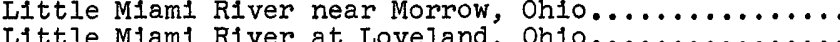

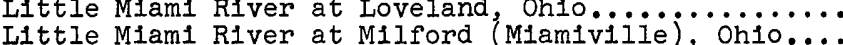

East Fork Little Miam1 River near Dodsonville, Oh1o East Fork I1ttle M1am1 River at W1lliamsburg, Ohio East Fork Little Mlami RIver near Bantam, Ohio.... Stonelick Creek:

Brushy Fork:

Paterson Run near Owensville, Ohio......

East Fork Little Miam1 River at Perintown, Ohio...

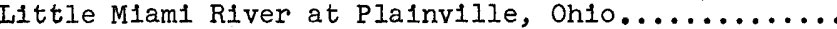
Licking River basin

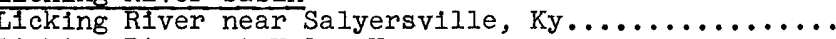

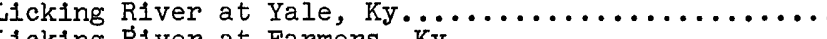

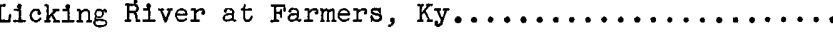

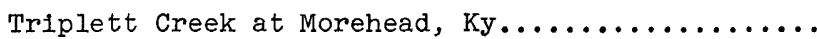

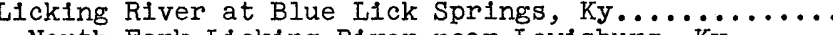
North Fork Licking R1ver near Lewisburg, Ky.......

Stoner Creek (head of South Fork Licking Riverj) at Paris, Ky.

South Fork Llcking River at Cynthiana, Ky ................... South Fork thayes (Falmouth)

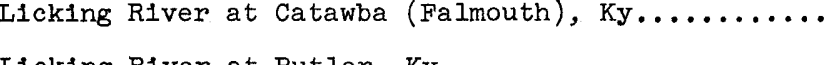

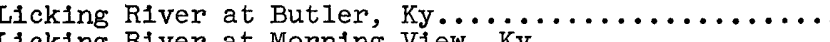

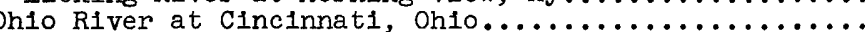

Mill Creek basin

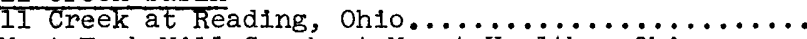
West Fork Mill Creek at Mount Healthy, Ohio...... West Fork Mill Creek near Greenhilis, Ohio........

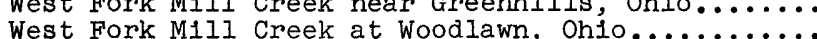

West Fork Mill Creek at Lockland (Arlington

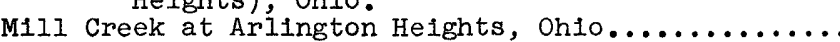

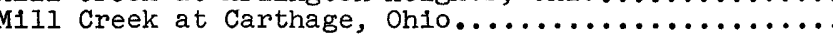

Mill Creek at Mitchell Avenue, Cincinnati, Ohio....

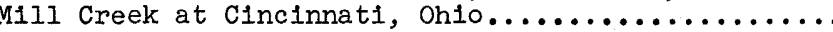

Great Miami River basin

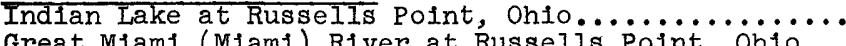
Bokengehalas (Buckiver at Russelis Point, Ohio...

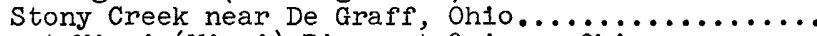
Great Miami (Miami) River at Quincy, Ohio..........

Great Miami (Miami) River at Sidney, Ohio............. 2615

*age helghts, or gage heights and discharge measurements only.

a Approximately.

c In reports of U.S. Weather Bureau.

e In reports of Mississippi River Commission.
30
32
219
964
1,145
1,203

219

91.4
237

330

3.34

1,713

, 713

140

714
831

47.9
.985

1,785

2,319

2,326
239

621

915

3,300

3,385

a3, 520

a 76,580

\section{0}

7.90
29.9

29.9
29.9

29.9
32.2

35.6

114

115

135

164

109

133

37.5
59.2

408

545

545
a 85

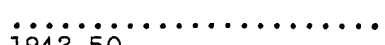
$1942-50$

$1952-$

$1903^{*}$.

1906* i $; 1918-20 *$

$1925-36 ; 1938-$

$1947-48$.

$1949-53$; $1960-$

\section{i}

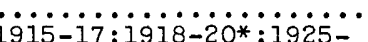

1914-15;1918-20*.

1938-

$1938-42$.

1915-*c; $1915-20 *$;

1928-31;1936-

$1941-$

$1938-59 \ldots \ldots \ldots \ldots \ldots$. . . .

$1924-26 ; 1938-$

$1953-$

$1917-^{*} \mathrm{c} ; 1938$

1915-16; 1917-20*;

1928-31.

1887-*c;1914*; 1915-17;

$1918-20 * ; 1928$ -

$1938-42$.

1.858-86*e; $1890-*$ c ; 1936 -

1938-

$1949-53$.

1945-53.

1938-57.

1912-16*.

1946-

1940-48.

1946-59*.

$\ldots \ldots \ldots$

$1957-$

$1946-49$

$1914-$ 


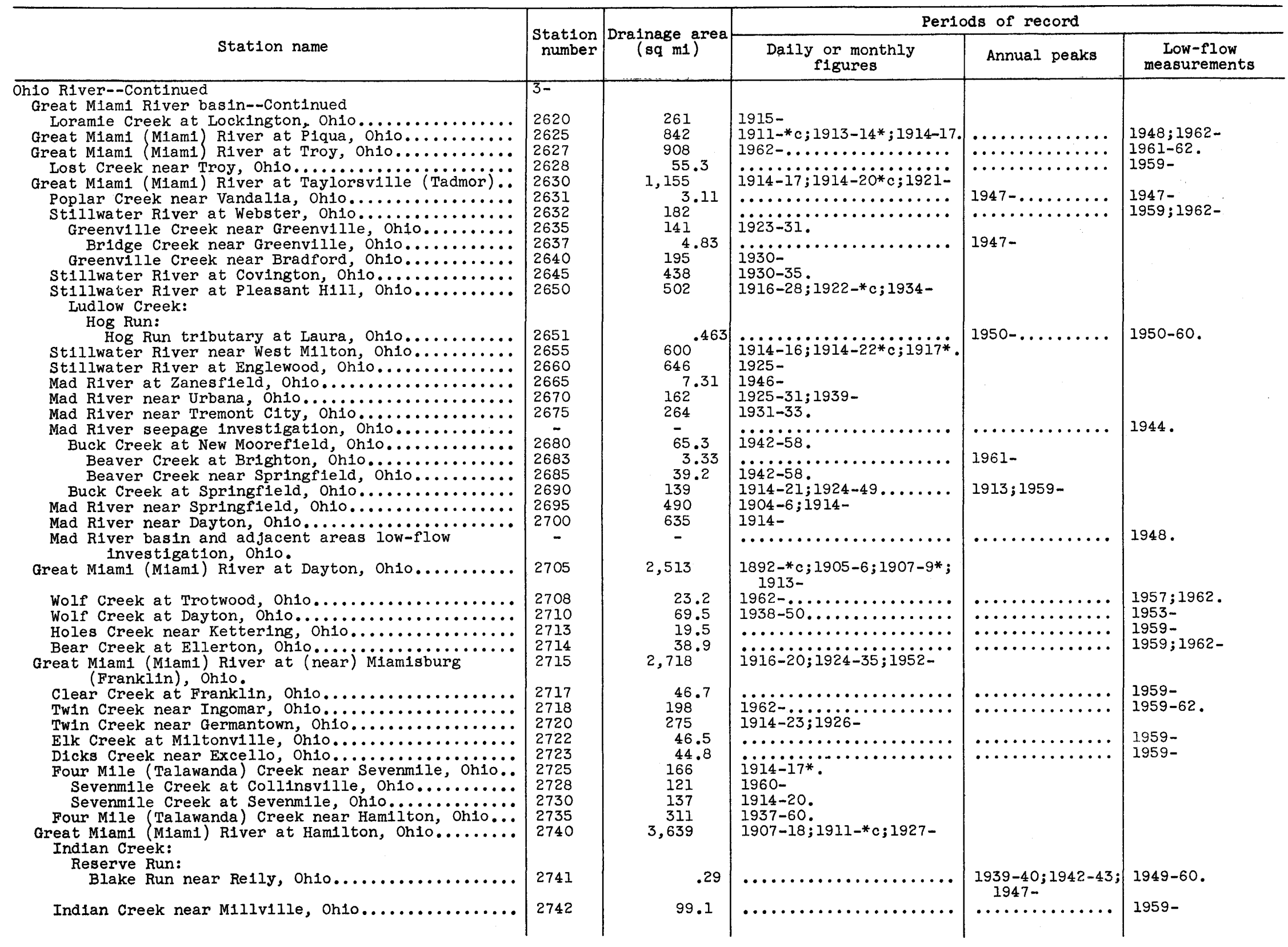


Great Miam1 (MIam1) River at Ven1ce, Oh10......... Great Miam1 (Mlami) River at New Baltimore, Ohio... Whitewater River:

Martindale Creek near Cambridge C1ty, Ind......

Whitewater (West Fork Whitewater) River near Alpine, Ind.

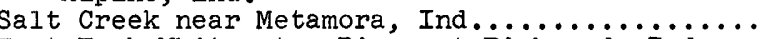
Eest Fork Whitewater River at Richmond, Ind....

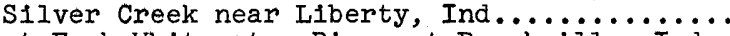
East Fork Whitewater River at Brookville, Ind...

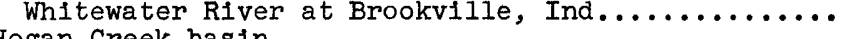
Hogan Creek basin Dilisboro, Ind.

Laughery Creek basin

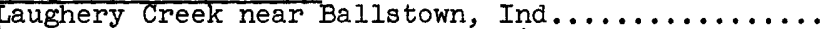
Versallies Lake near Versallies (Versallies State Park), Ind.

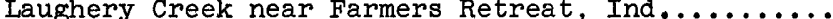
Kentucky River basin

North Fork Kentucky River at Whitesburg, Ky.......

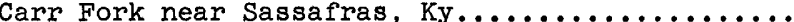

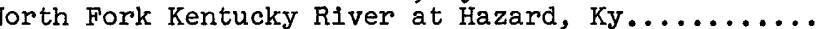
Troublesome Creek:

Bear Branch near Noble, Ky . .

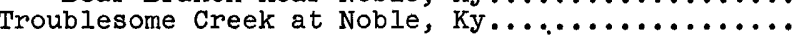

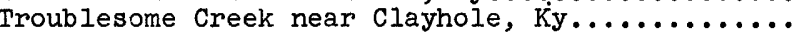

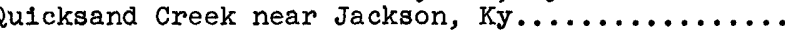

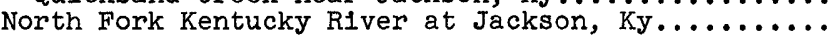

North Fork Kentucky River near Airdale, Ky........ Middle Fork Kentucky River near Hyden, Ky.......

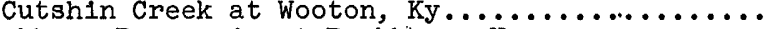

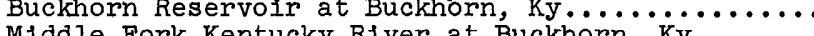

Middle Fork Kentucky RIver at Buckhorn, Ky,.....

Kentucky River:

South Fork Kentucky River at Oneida, Ky..........

South Fork Kentucky River at Booneville, Ky......

Kentucky River at lock 14, at Heidelberg, Ky.......

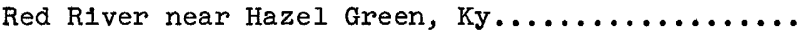

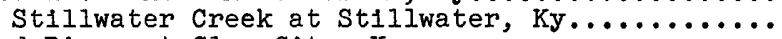

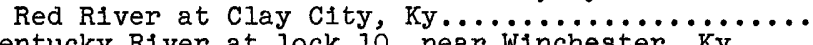

Kentuky River at lock

Kentucky Rlver at lock 8 , near Camp Nelson, Ky.......

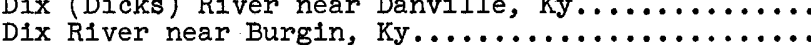

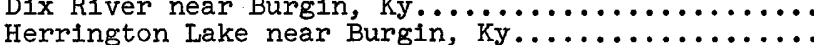

Herrington Lake near Burgin, Ky $\ldots \ldots \ldots \ldots \ldots \ldots$

Kentucky River at lock 6, near Salvisa (at Warwickj): 2870

* Gage heights, or gage helghts and discharge measurements only.

c In reports of U.S. Weather Bureau.

$f$ In files of Geological Survey.

$g$ Part of drainage area is noncontributing or does not contribute directly to surface runoff.

In files of Kentucky Utilities Co. \begin{tabular}{l|l}
3,790 & $1914-27 ; 1932-33$
\end{tabular}

about 57

539

118

bout 9

382

1,239

38.2

about 37

165.0

248

66.4

60.

466

$2.21 \quad 1954-$

177 1949-

187

1,101

1,294

202

61.3

420

5.37

486

722
657

65.8

362

3,955

4,414

318
395

439

85,036

85,102 $\ddot{i} \ddot{2} \dot{8}=$

ị̈9-

1954-

1915-17; 1918-20*; 1923-

196

$i 957-f$

1940-

$963-$

925-*c;1940-

1929-31.

( $1904-7 ; 1913-21$;

1935-)*c;1928-31;

$1936-37 ; 1938-$

$1929-31$; 1939-42.

$1957-$

$1957-$

$1960-$

1930-3? ; 1939 -

…………

$1902-* c ; 1925-31$

$1936-37$; $1938-$

$1954-$

$1930-32$; 1938 -

$1907-$

$1939-$

$1905 *$; $1942-$

$1910-22$

1925-h;1950-

$1901-* c ; 1925-27$.

$1925-$

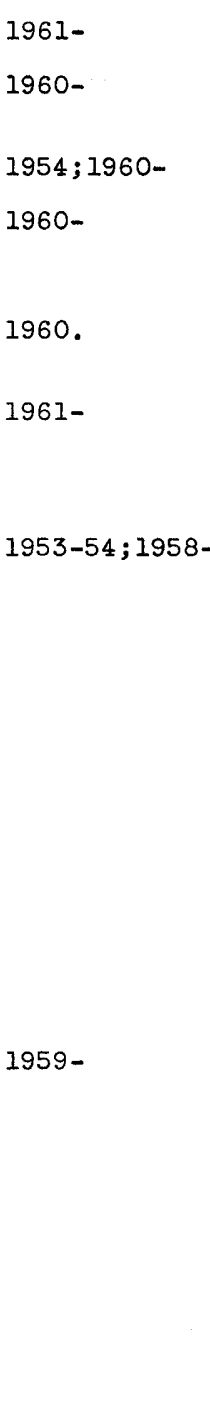

1957

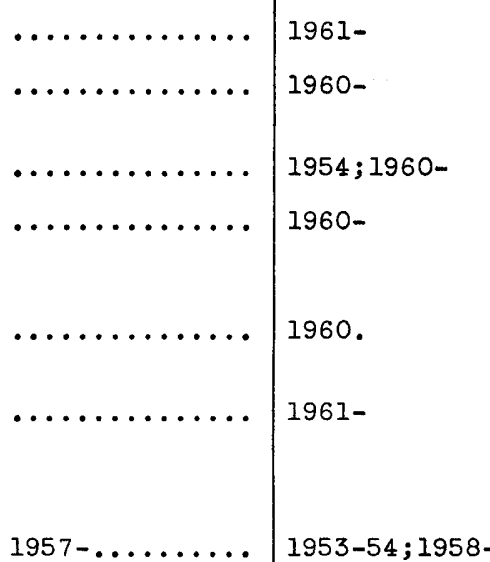

1957

1959

.
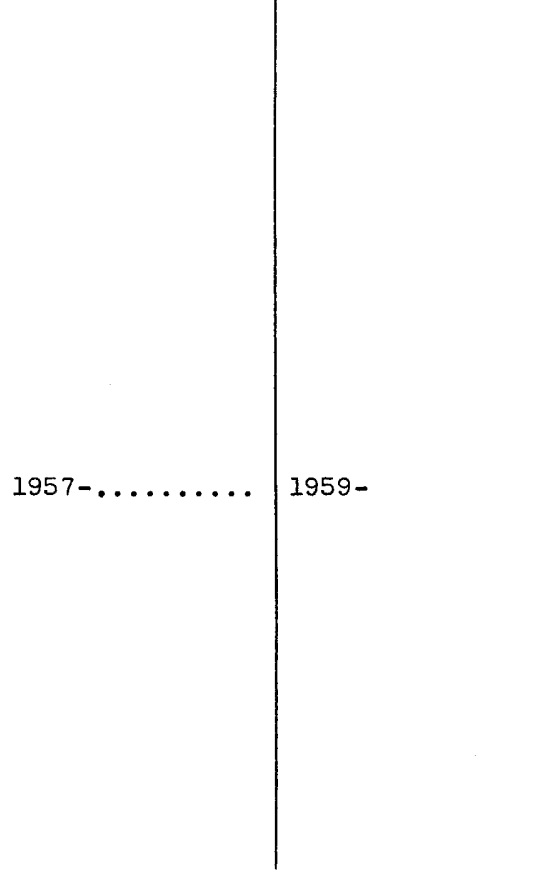


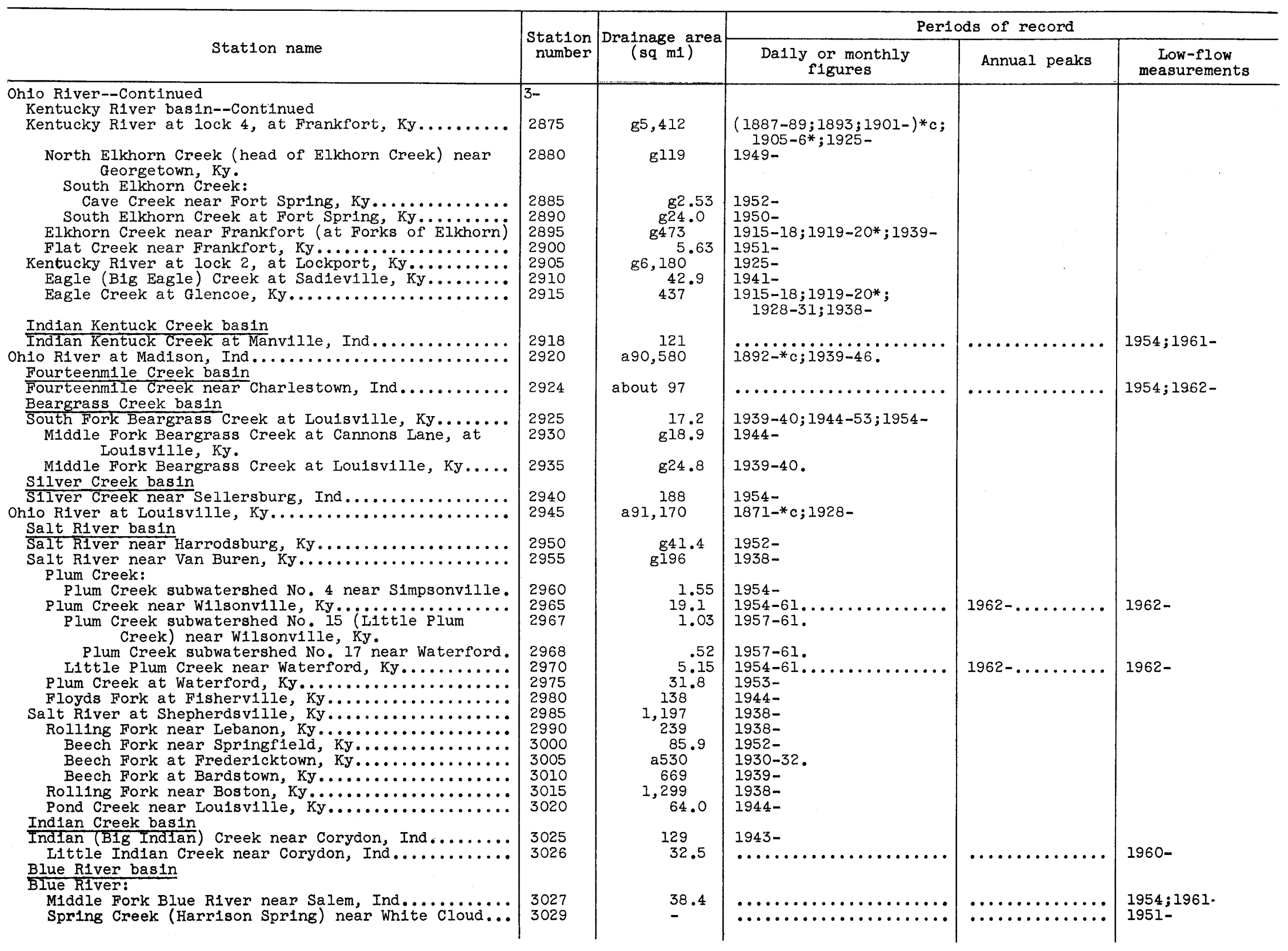




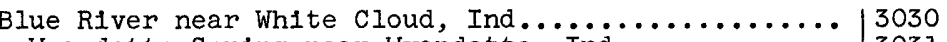

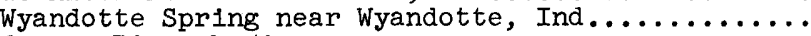
Anderson River basin

Middle Fork Anderson River at Bristow, Ind........

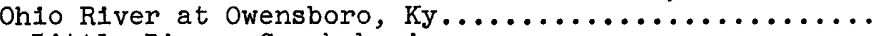

Little Pigeon Creek bas

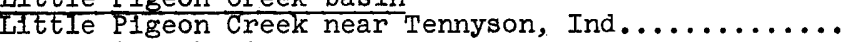
Green River basin

Green River:

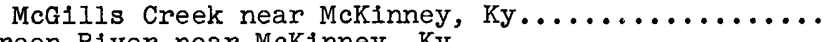
Green River near McKinney, Ky....

Green River near Mount Salem, Ky $\ldots \ldots \ldots \ldots \ldots \ldots \ldots$

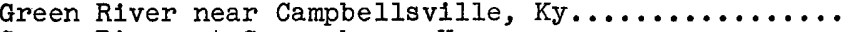

Green River at Greensburg, Ky.....

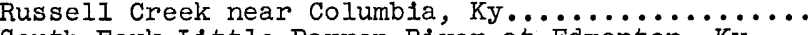

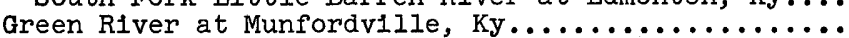

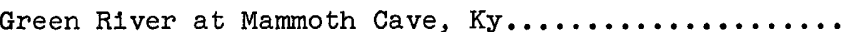
North Fork Nolin River:

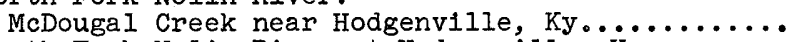

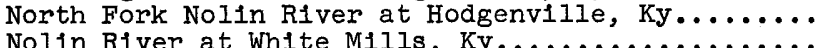

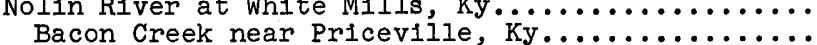

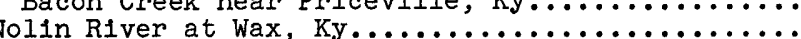
Nolin River at wax, Ky $\ldots \ldots \ldots \ldots \ldots \ldots \ldots$

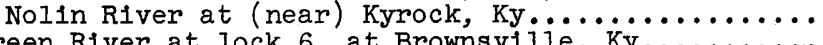

Green River at lock 6 , at Brownsville, Ky.........

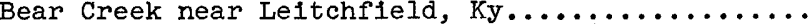
Barren RIver:

Salt Lick Creek:

Long Fork:

Whiteoak Creek:

Town Creek at Lafayette, Tenn...................... 3123

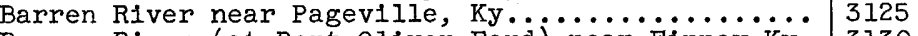

Barren River (at Port Oliver Ford) near Finney, Ky. 3130

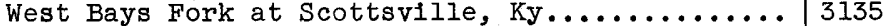

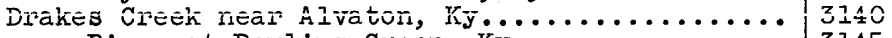

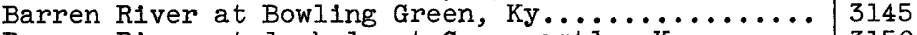

Barren Rlver at lock 1, at Greencastle, Ky........ 3150

Green River at lock 4 , at Woodbury, Ky....................... 3155

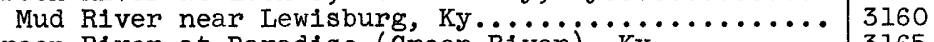

Green River at Paradise (Green River), Ky................. 3165

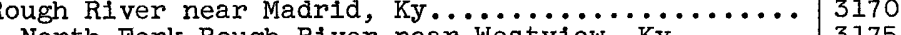

North Fork Rough River near westview, Ky................ 3175

Rough River near (at) Falls of Rough, Ky.........
Rough River Reservolr near Falls of Rough, Ky.....

Rock Lick Creek near Glen Dean, Ky............... 3182

Rough River at (near) Falls of Rough, Ky......... 3185

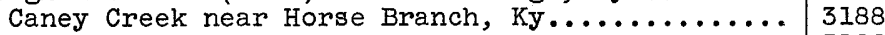

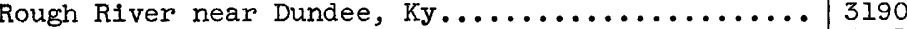

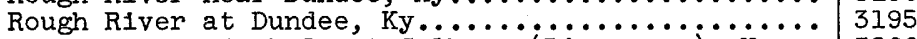
Green River at lock 2, at Calhoun (Ilivermore), Ky... 3200

* Gage helghts, or gage helghts and discharge measurements only.

a Approximately.

c In reports of U.S. Weather Bureau.

$f$ In files of Geological Survey.

g Part of drainage area is noncontributing or does not contribute directly to surface runoff.
$1954-61$

$1961-$

$1940-54 ; 1954-60 * f$.

$1943-47$.

1961-

1951-

22.4 1951-

36.3

682

736

g188

18.3

$1953-61$

$1939-$

$1941-$

1915-22 ; $1924-* c: 1927-31$ $1936-$

g1, 983

5.34

836.4

g337

g600

8703

8707

82,762

30.8

$1938-50$; $1950-* f$

1953-

$1941-$

$1959-$

1936-62.

$1930-32 ; 1939-50 ; 1960-$

$1924-31 ; 1936-37 ; 1938-$

$1949-$

1.85

g531

8940

$\tilde{5}^{4} 70^{\circ}$

g1, 848

g1, 966

g90.5

6,182

$\mathrm{g} 225$
$\mathrm{~g} 42.0$

g454

8454
20.1

20.1

$\mathrm{g} 504$
124

124
8757

815

g7, 564

\section{$1939-63$.}

$1941-50 ; 1960-$

$1950-$

$1901-* c ; 1938-$

$1924-31 ; 1936-37$

1917-*c;1936-

1939-

$1939-50 ; 1959-$

$1938-59$

1939-56.

$1959-$

$1956-$

1939-

1956-

$1939-$

1938-40.

1930-

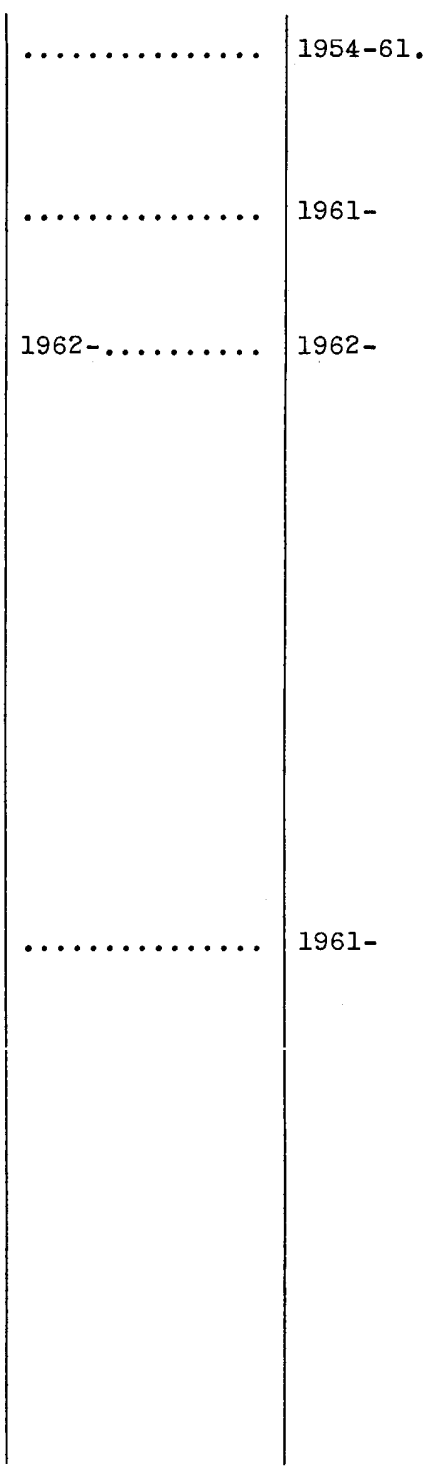




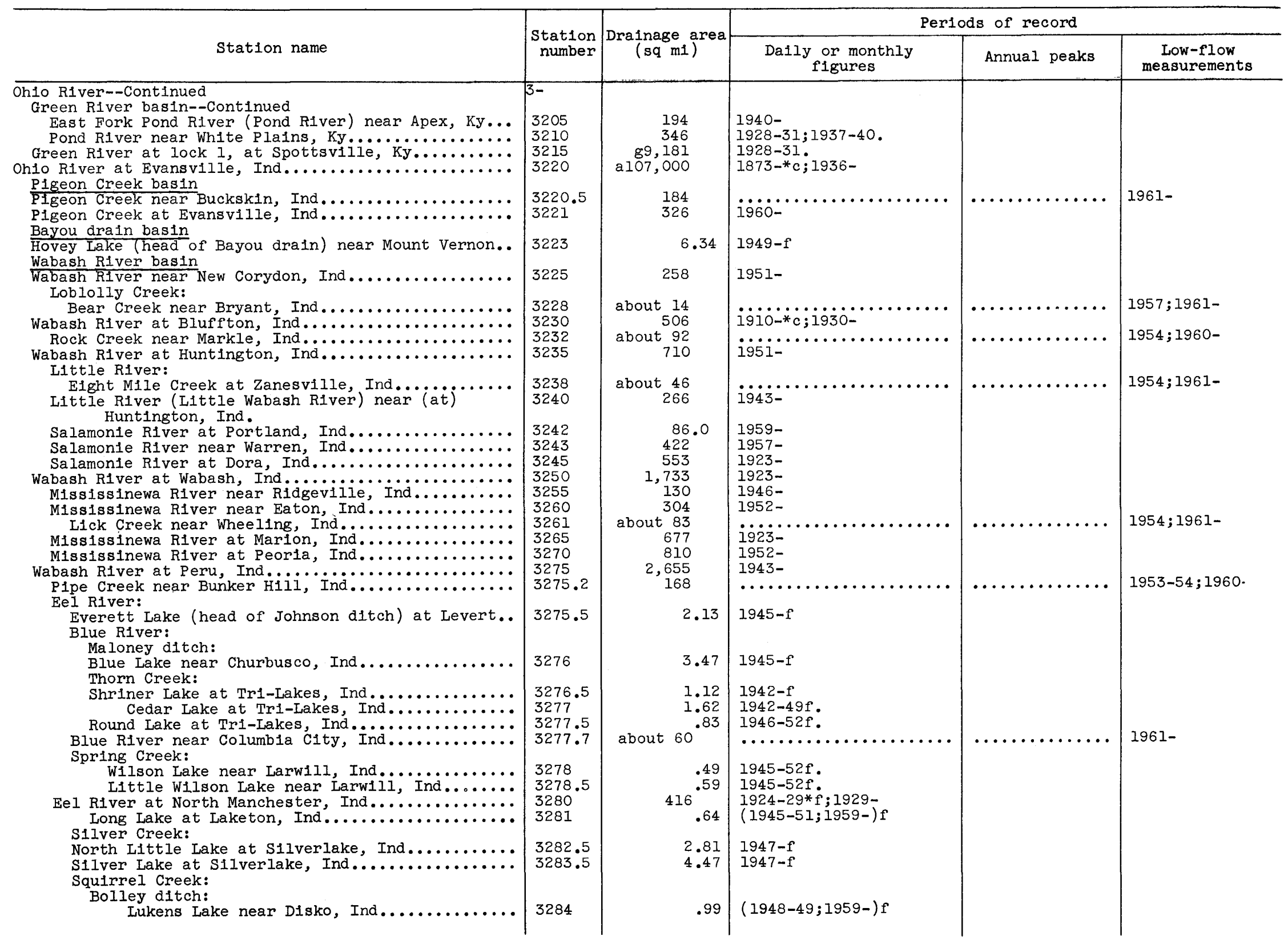

reen River basin--Continued

East Fork Pond River (Pond River) near Apex, Ky... Pond River near White Plains, Ky $\ldots \ldots \ldots \ldots \ldots$

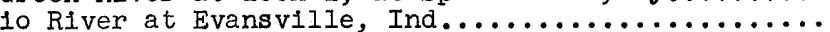

(n) ittle River Wabash River) near (at) Huntington, Ind.

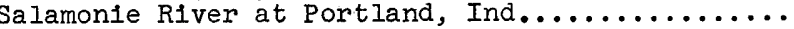

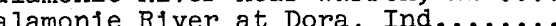
at Tolumbia city, Ind. quirrel Creek:

Iukens Lake near Disko, Ind............ 3284

(1) 
Eel River near Logansport, Ind............ Wabash River at Logansport, Ind..............

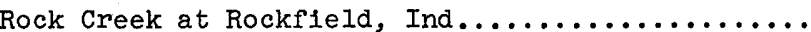

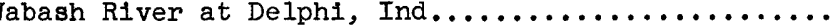
Deer Creek:

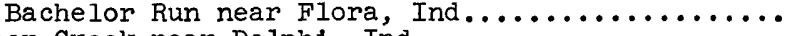

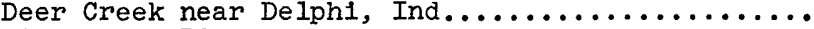
Tippecanoe River:

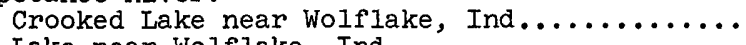

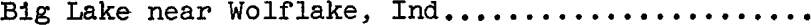

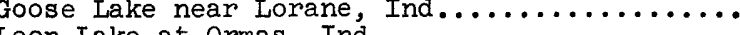

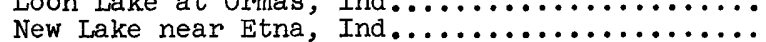
Tippecanoe River:

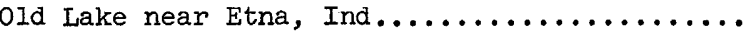

Smalley Lake near Washington Center, Ind......... Gilbert Lake near Washington Center, Ind...... Baugher Lake near washington Center, Ind.......... Wilmot Pond (Rider Lake) at W1lmot, Ind.......... Webster Lake at North Webster, Ind.

lder ditch (head of Grassy Creek):

Troy Cedar Lake (head of Cedar Lake Branch) near Lorane, Ind.

Robinson Lake near Pierceton, Ind.........

Ridinger Lake (continuation of Elder ditch)

near Pierceton, Ind.

Kuhn Lake near North Webster, Ind..........

Grassy Creek:

B1g Barbee Lake (Barbee Lake) near North

$$
\text { Webster, Ind. }
$$

Little Barbee Lake near North Webster, Ind......

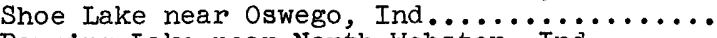
Banning Lake near North Webster, Ind........

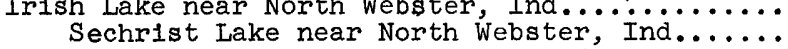

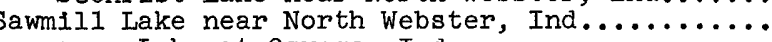

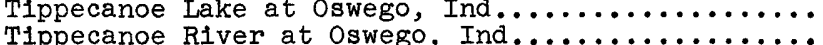

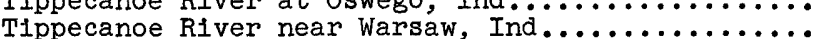

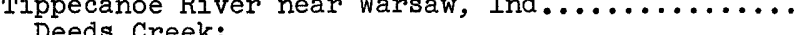
Deeds Creek:

Big Chapman Lake (head of Heeter ditch) near Warsaw, Ind.

Little Chapman Lake near Warsaw, Ind.........

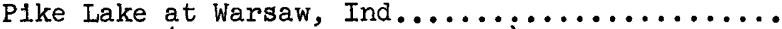
Fish Lake (head of Walnut Creek) near Warsaw.....

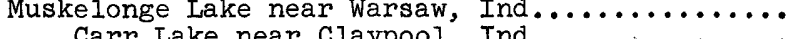

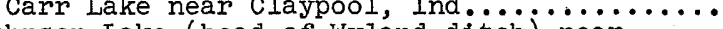
Johnson Lake (head of Wyland ditch) near Pierceton, Ind.

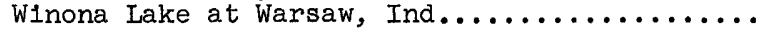

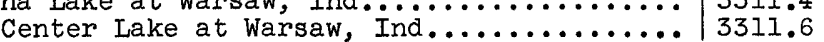

791
3,751

3290

3293 3295

3296 3297

3300.2

3300.6

3300.8

3301

3301.2

3301.4

3301.8

3302

3302.2

3302.4

3302.6

3302.8

3303

3303.2

3303.4

3303.6

3303.8

3304

3304.2

3304.4
3304.6

5304.8

3310

3310.1

3310.2

3310.4

3310.6

3310.8

3311

3311.2 about 81

out 13

278

1.32

6.77

11.42

.49

3.13

32.6

1.39

36.4

54.0

5.51

6.95

34.9

3.74

44.8

48.8

.48

50.8

.42

51.9

115

115

125

4.59

7.78
40.4

40.4
3.59

1954-f

11.1
2.56

5.42

32.1

$.751945-f$

* Gage heights, or gage helghts and discharge measurements only.

a Approximately.

c In reports of U.S. Weather Bureau.

In files of Geological Survey.

Part of drainage area is noncontributing or does not contribute directly to surface runoff. 


\begin{tabular}{|c|c|c|c|c|c|}
\hline \multirow[b]{2}{*}{ Station name } & \multirow{2}{*}{$\begin{array}{r}\text { Station } \\
\text { number }\end{array}$} & \multirow{2}{*}{$\frac{\text { Drainage area }}{\text { (sq } \mathrm{mi})}$} & \multicolumn{3}{|c|}{ Periods of record } \\
\hline & & & $\begin{array}{c}\text { Daily or monthly } \\
\text { figures }\end{array}$ & Annual peaks & $\begin{array}{l}\text { Low-flow } \\
\text { measurements }\end{array}$ \\
\hline 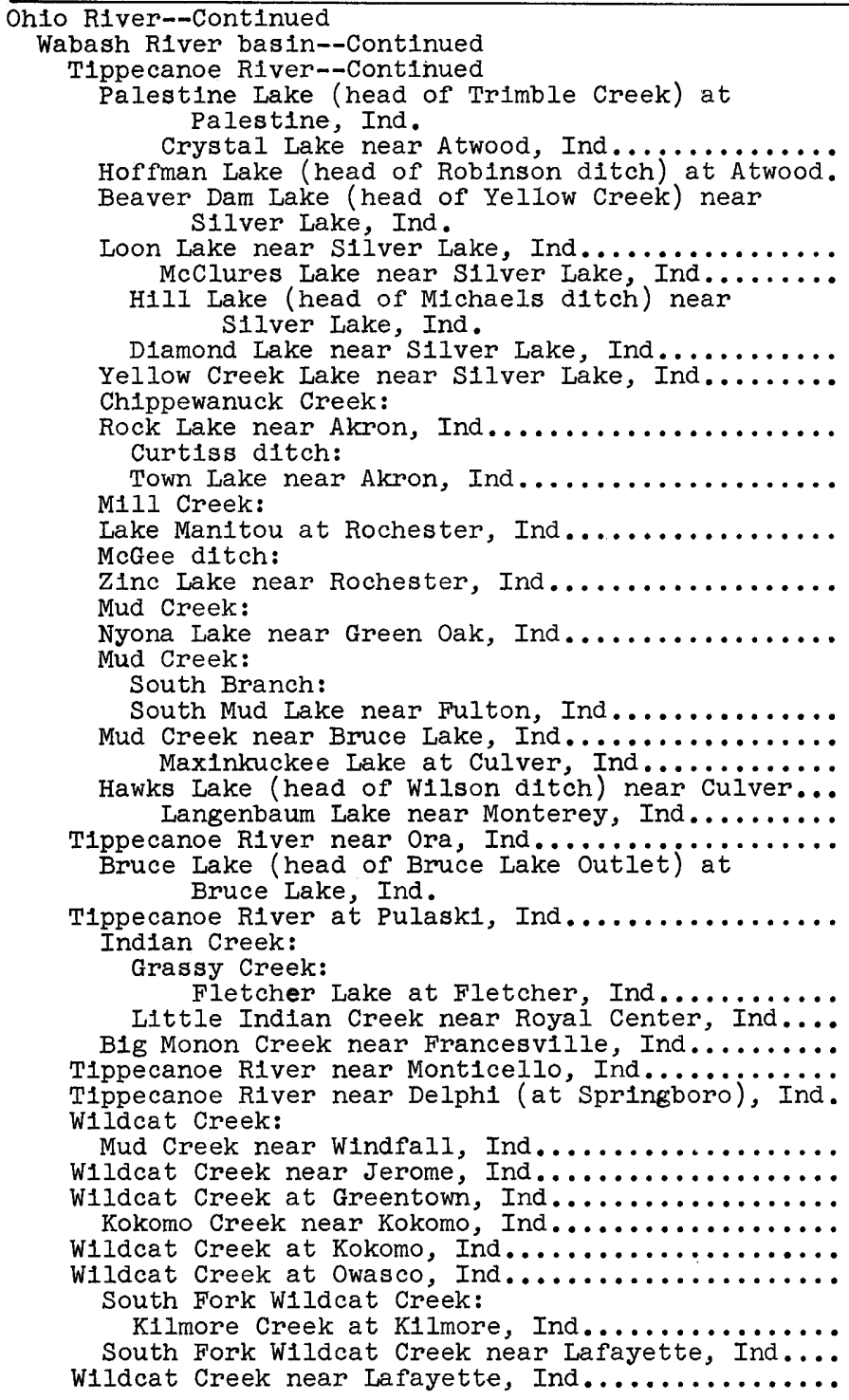 & $\begin{array}{l}3311.8 \\
3312 \\
3312.2 \\
3312.4 \\
\\
3312.6 \\
3312.8 \\
3313 \\
\\
3313.2 \\
3313.4 \\
3313.6 \\
3313.7 \\
3313.8 \\
\\
3313.9 \\
3314 \\
\\
3314.2 \\
3314.3 \\
3314.4 \\
3314.6 \\
3314.8 \\
3315 \\
3317 \\
3320 \\
3322 \\
3323 \\
3324 \\
3325 \\
3330 \\
3334 \\
3334.5 \\
3335 \\
3336 \\
3337 \\
3340 \\
3343 \\
3345 \\
3350\end{array}$ & $\begin{array}{r}29.9 \\
.38 \\
7.14 \\
1.89 \\
2.70 \\
.45 \\
.56 \\
5.35 \\
8.50 \\
1.78 \\
1.74 \\
38.1 \\
.26 \\
4.74 \\
\text { about } 69 \\
9.48 \\
10.2 \\
.98 \\
839 \\
5.19 \\
1,066 \\
\text { about } 62 \\
246 \\
791 \\
146 \\
148 \\
162 \\
24.3 \\
245 \\
390 \\
35.0 \\
145 \\
1,710 \\
857 \\
75 \\
10\end{array}$ & $\begin{array}{l}1954-f \\
1945-51 f . \\
1945-52 f . \\
1947-52 f . \\
1947-52 f . \\
1945-52 f . \\
1952-f \\
1954-f \\
1945-52 f . \\
1949- \\
1949-50 f . \\
1942-f \\
1952-54 f . \\
1945-f \\
1945-f \\
190 \ldots \ldots \\
1954-f \\
1954-f \\
1943- \\
1943-52 f . \\
1927-31 . \\
1945-53 f . \\
1959- \\
1959- \\
1931- \\
1903-6 ; 1908 ; 1939- \\
1930 \ldots \ldots \\
1944-61 . \\
1959- \\
1955- \\
1943- \\
1930 \ldots \\
1954-\end{array}$ & $\ldots \ldots \ldots$ & 1954 ; $1960-$ \\
\hline
\end{tabular}




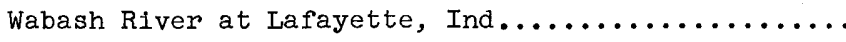

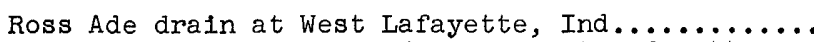
Ross Ade drain below Purdue, at West Lafayette....

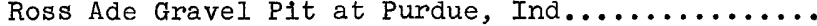

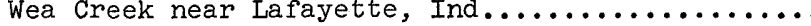

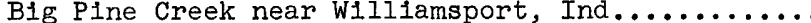

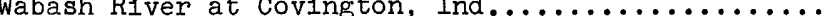
Vermilion River:

Vermilion River tributary near Paxton, Ill.....

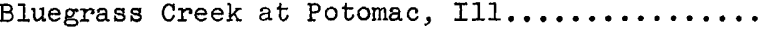

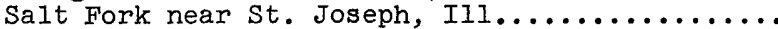
Saline Branch:

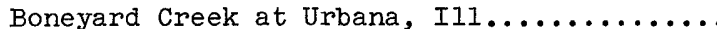
Saline Branch (West Branch Salt Fork) at Urbana, Ill.

Salt Fork (Salt Fork Vermilion River) near Homer Salt Fork tributary near Catlin, Ill.........

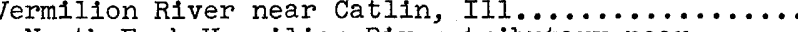
North Fork Vermilion River tributary near Danville, III.

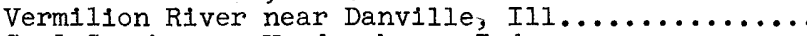

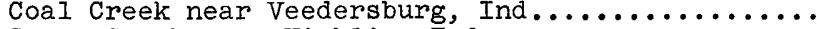

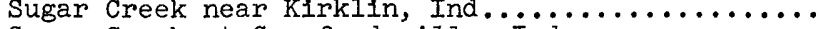

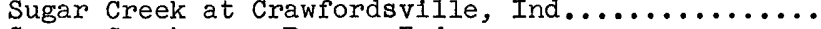

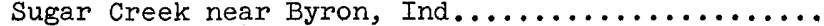

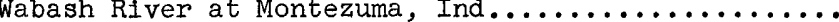

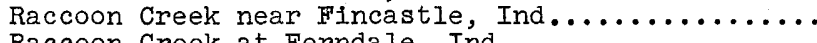

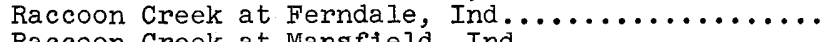

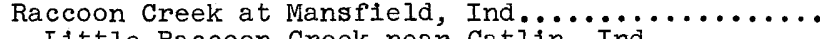

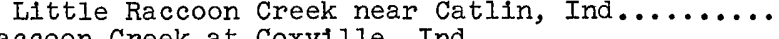

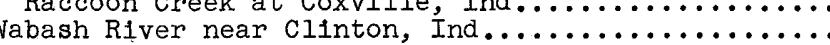

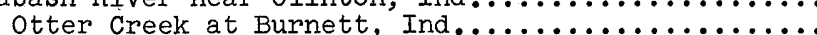

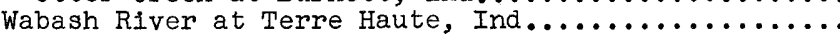

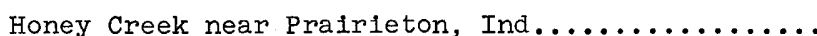
Big Creek:

Big Creek tributary near Dudley, Ill..........

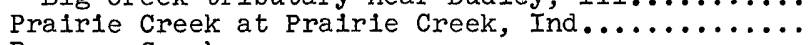
Raccoon Creek:

Raccoon Creek tributary near Annapolis, III....

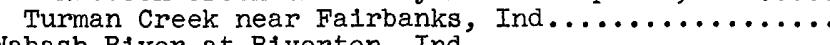

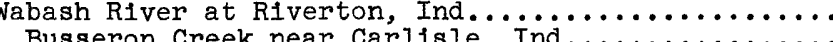

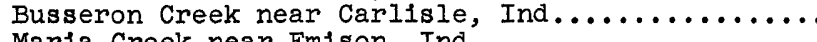

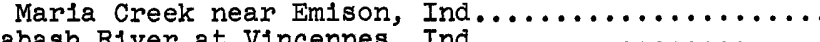

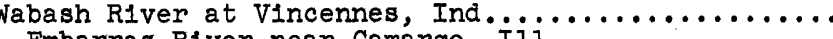

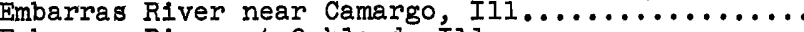
Embarras River at Oakland,

Embarras RIver near Diona, Ili..... Embarnas River tributary near Greenup iija..... Muddy Creek:

Muddy Creek tributary at Woodbury, III.......

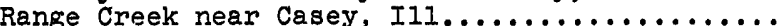

\begin{tabular}{|l|}
3355 \\
3356 \\
3356.2 \\
3356.4 \\
3356.7 \\
3357 \\
3360 \\
3361 \\
3365 \\
3369 \\
3370 \\
3375 \\
3380 \\
3381 \\
3385 \\
3388 \\
3390 \\
3391 \\
3392 \\
3395 \\
3400 \\
3405 \\
3408 \\
3409 \\
3410 \\
3412 \\
3413 \\
3414 \\
3414.5 \\
3415 \\
3416 \\
3417 \\
3418 \\
3419 \\
3419.5 \\
3420 \\
3425 \\
3427 \\
3430 \\
3434 \\
3435 \\
3440 \\
3442.5 \\
3444.25 \\
3445
\end{tabular}

$1901-2 ; 1903 * ; 1913-* 0 ;$

103
329

329
, 208

1.05

134

4.70
71.4

71.

344

959
1.42

1,279

about 76

about 41

509

668

a11, 100

132
215

240

133

440
700

11,700

about 69

a 12,200

about 86

about 24

.903

about 69

a 13,100

about 88

a.13, 700

185
535
903

.081

.091

036

ïзㅛ-

1943-

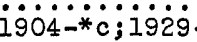

$1960-$

$1909-12$; 1914-15.

$1938-40$; 1944-47

$7.60 \mid \dot{9} \dot{5} 0$

$1956-$

$1941-* f$

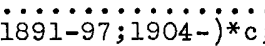
$1902-3^{*} ; 1905-6 ; 1927-$

* Gage helghts, or gage helghts and discharge measurements only. a Approximately.

c In reports of U.S. Weather Bureau.

In flies of Geological Survey.

1 In reports of Indiana Department of Conservation. 


\begin{tabular}{|c|c|c|c|c|c|}
\hline \multirow[b]{2}{*}{ Station name } & \multirow{2}{*}{$\begin{array}{r}\text { Station } \\
\text { number }\end{array}$} & \multirow{2}{*}{$\begin{array}{c}\text { Drainage area } \\
\text { (sq ms })\end{array}$} & \multicolumn{3}{|c|}{ Periods of record } \\
\hline & & & $\begin{array}{c}\text { Daily or monthly } \\
\text { figures }\end{array}$ & Annual peaks & $\begin{array}{c}\text { Low-flow } \\
\text { measurements }\end{array}$ \\
\hline 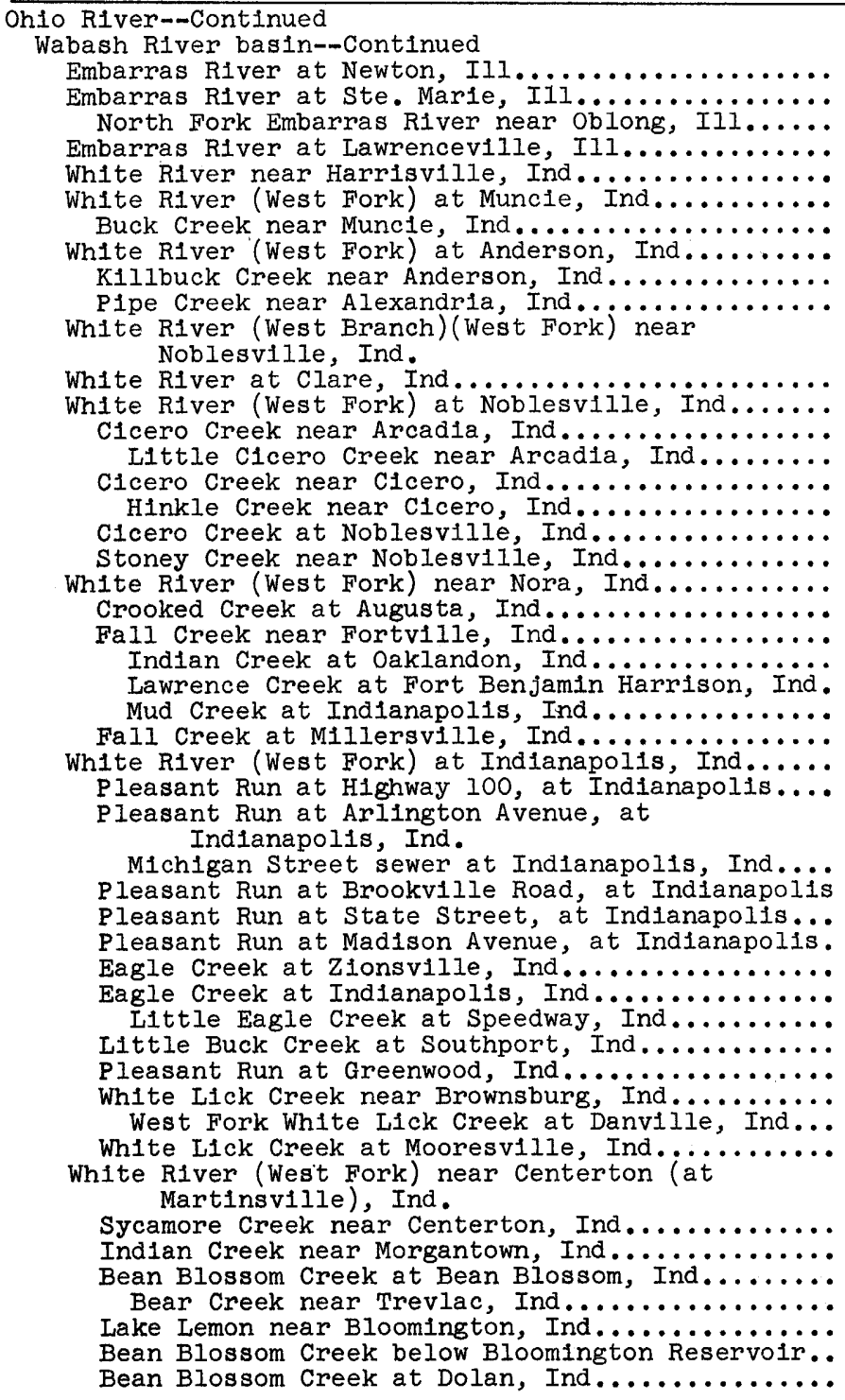 & $\begin{array}{l}3450 \\
3455 \\
3460 \\
3465 \\
3467 \\
3470 \\
3475 \\
3480 \\
3481 \\
3483 \\
3485 \\
\\
3488 \\
3490 \\
3495 \\
3497 \\
3500 \\
3501 \\
3505 \\
3507 \\
3510 \\
3513 \\
3515 \\
3519 \\
3520 \\
3522 \\
3525 \\
3530 \\
3531.1 \\
3531.2 \\
3531.4 \\
3531.6 \\
3531.7 \\
3531.9 \\
3532 \\
3535 \\
3536 \\
3536.3 \\
3536.5 \\
3536.7 \\
3537 \\
3538 \\
3540 \\
\\
3541 \\
3542 \\
3545 \\
3550 \\
3554 \\
3555 \\
3560 \\
\end{array}$ & $\begin{array}{c}\text { a1, } 390 \\
\text { a1, } 513 \\
319 \\
\text { a2, } 260 \\
\text { about } 19 \\
242 \\
36.7 \\
401 \\
\text { about } 96 \\
\text { about } 38 \\
814 \\
830 \\
837 \\
131 \\
44.7 \\
196 \\
16.3 \\
219 \\
\text { about } 51 \\
1,200 \\
7.22 \\
172 \\
\text { about } 18 \\
2.86 \\
42.5 \\
313 \\
1,627 \\
3.31 \\
7.67 \\
\text { a2 } \\
10.3 \\
13.6 \\
21.0 \\
102 \\
179 \\
18.6 \\
\text { about } 9 \\
\text { about } 5 \\
\text { about } 30 \\
28.9 \\
212 \\
2,435 \\
\text { about } 20 \\
14.6 \\
7.00 \\
64.0 \\
64.0 \\
100 \\
10\end{array}$ & 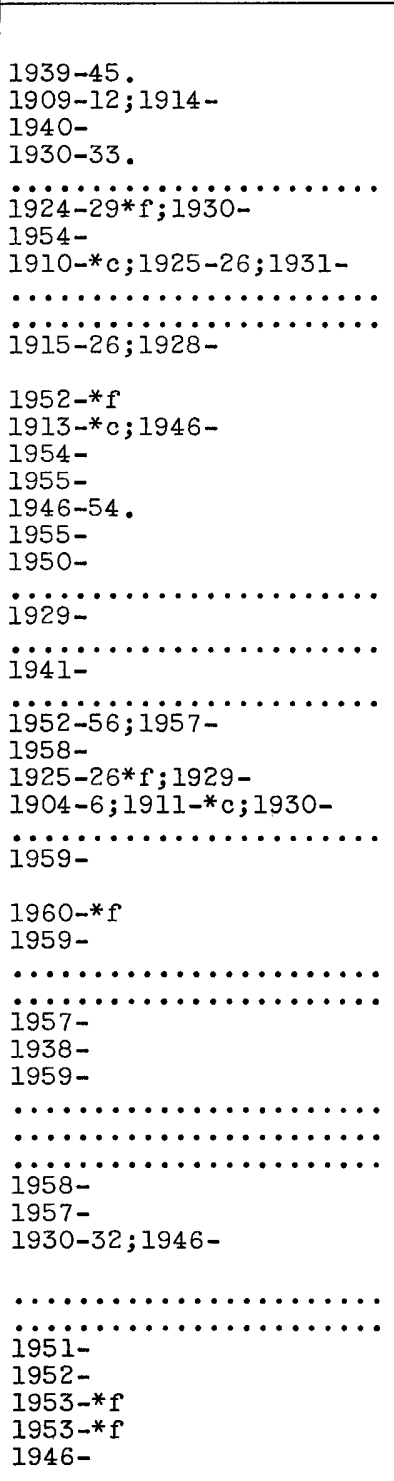 & $\begin{array}{l}1960-* f \\
1960-* f \\
1960-* f\end{array}$ & $\begin{array}{l}1946 ; 1960- \\
1960- \\
1960-\end{array}$ \\
\hline
\end{tabular}


Bean Blossom Creek near Bloomington, Ind....... White River (West Fork) at Spencer, Ind.......................... Big Walnut Creek (head of Eel River) near

$$
\text { Barnard, Ind. }
$$

Big Walnut Creek (Eel River) near Reelsville, Ind

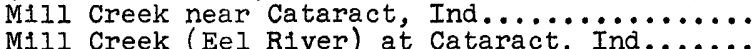
Mill Creek (Eel River) at Cataract, Ind.......

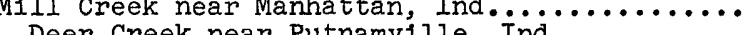

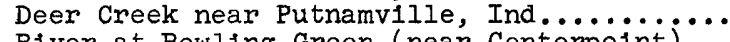
Eel River at Bowling Green (near Centerpoint)...

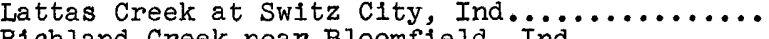

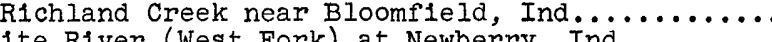

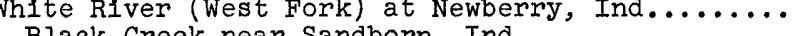
Black Creek near Sandborn, Ind.

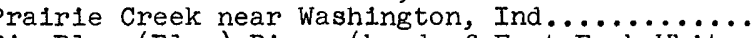

Big Blue (Blue) River (head of East Fork White River) at Carthage, Ind.

Little Blue River near Rays Crossing, Ind....

Big Blue (Blue) River at Shelbyville, Ind.......

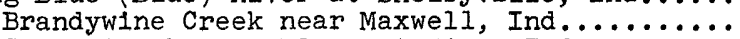

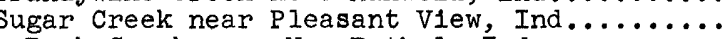

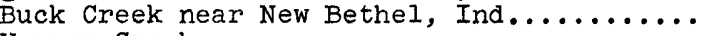
Youngs Creek:

Hurricane Creek at Frankin, Ind........

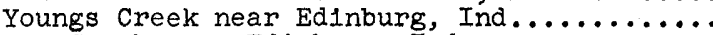

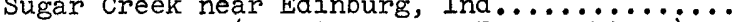
Driftwood River (continuation of Blue River) near Edinburg, Ind.

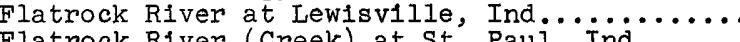

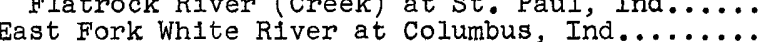

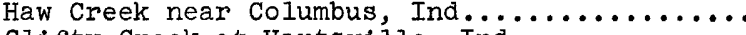

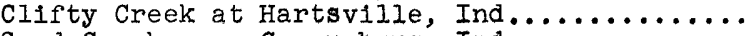

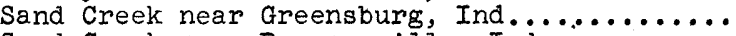

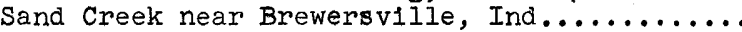

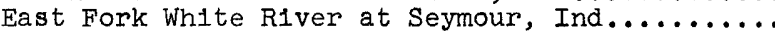

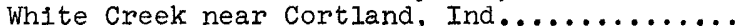
Graham Creek (head of Muscatatuck River) near Vernon, Ind.

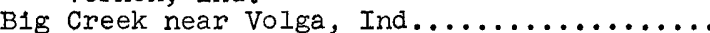

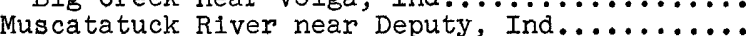

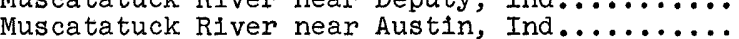

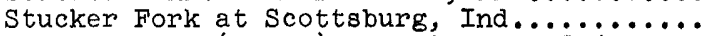
Stucker Fork (Creek) near Austin, Ind....... Vernon Fork:

Brush Creek near Nebraska, Ind.......... 3680 Vernon Fork (North Fork of Vernon Fork) near Butlerville, Ind.

Vernon Fork at Vernon, Ind.............. 3695

Vernon Fork near Crothersvilie, Ind........ 3700

Muscatatuck River near Tampico, Ind............. 3705

Starve Hollow Lake near Vallonia, Ind...... 3709

Muscatatuck RIver near Vallonia, Ind............. 3710
112
2,980
about 25
about 120

338

338
241

248

292

59.0

about 32

about 96

4,696
101
117

117

187

about 94

about 25

121
49.5

about 13

109
462

462
1,054

about 49

298

1,692

about 58.8

about 9

156
233

about 94

94
77.6

about 96

296

365

about 74

126

$$
\begin{aligned}
& 11.7 \\
& 87.3
\end{aligned}
$$

201

395

1,060 $1,144^{\circ} \mid$\begin{tabular}{l|l}
1932. \\
\end{tabular}

1931-32.

1925

(1)

1949

$1949-$

$1903-6^{*}$

1954

$1931-$

.....................

1928 -

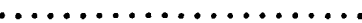

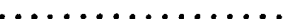

1950-

i9̈43-

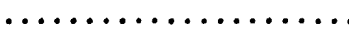

.

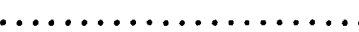

$1942-$

$1942-$

1940-

ig̈30-

1947 -

$\ddot{194 \dot{8}-}$

ï்ن்-

$1923-27 * f ; 1927$

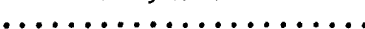

1955-

$\ddot{1947}=$

1932-

$\dot{193} \dot{3}$.

1955-

1942-

1939-

1939 .
19601961-

$\ldots$

$1954 ; 1960-$ 1960-

$1960-$

$1954 ; 1960-$

$1960-$

$1960-$

1954 ; $1960-$

1960-

1960-

$1954 ; 1960$

$1960-$

1960-

$1954 ; 1961$

$1954 ; 1961-$

1961-

* Gage helghts, or gage helghts and discharge measurements only.

Approximately.

In reports of U.S. Weather Bureau.

$f$ In files of Geological Survey. 


\begin{tabular}{|c|c|c|c|c|c|}
\hline \multirow[b]{2}{*}{ Station name } & \multirow{2}{*}{$\begin{array}{r}\text { Stat1on } \\
\text { number }\end{array}$} & \multirow{2}{*}{$\begin{array}{c}\text { Drainage area } \\
(\mathrm{sq} \mathrm{mI})\end{array}$} & \multicolumn{3}{|c|}{ Periods of record } \\
\hline & & & $\begin{array}{c}\text { Dally or monthly } \\
\text { figures }\end{array}$ & Annual peaks & $\begin{array}{l}\text { Low-flow } \\
\text { measurements }\end{array}$ \\
\hline 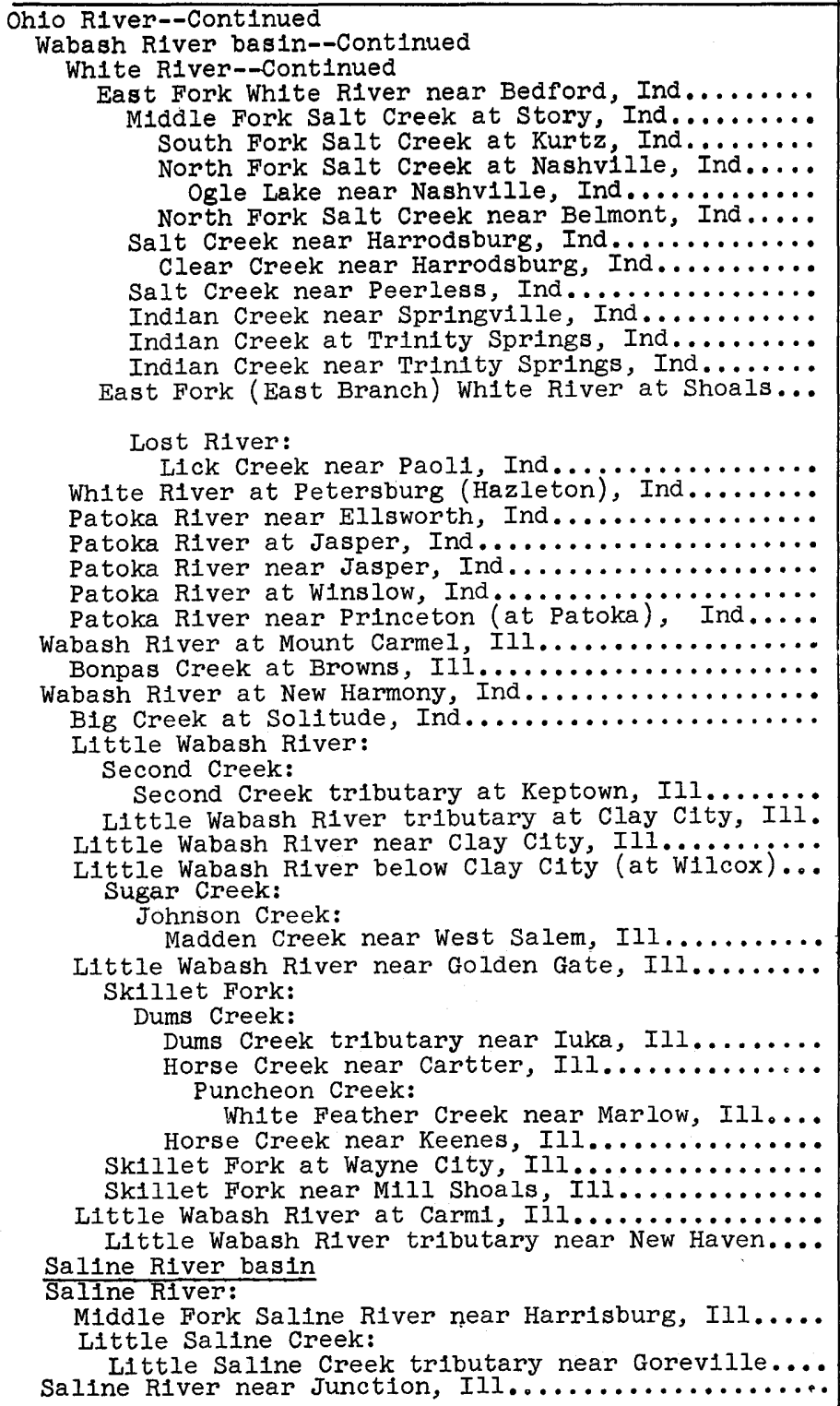 & $\begin{array}{l}3715 \\
3715.5 \\
3716 \\
3716.5 \\
3717 \\
3720 \\
3725 \\
3727 \\
3730 \\
3732 \\
3733 \\
3733.2 \\
3735 \\
\\
3736 \\
3740 \\
3745 \\
3755 \\
3760 \\
3763 \\
3765 \\
3775 \\
3780 \\
3785 \\
3786 \\
\\
3786.5 \\
3789.8 \\
3790 \\
3795 \\
\\
3796.5 \\
3800 \\
\\
3803 \\
3804 \\
3804.5 \\
3804.75 \\
3805 \\
3810 \\
3815 \\
3816 \\
\\
3820 \\
3820.25 \\
3825\end{array}$ & 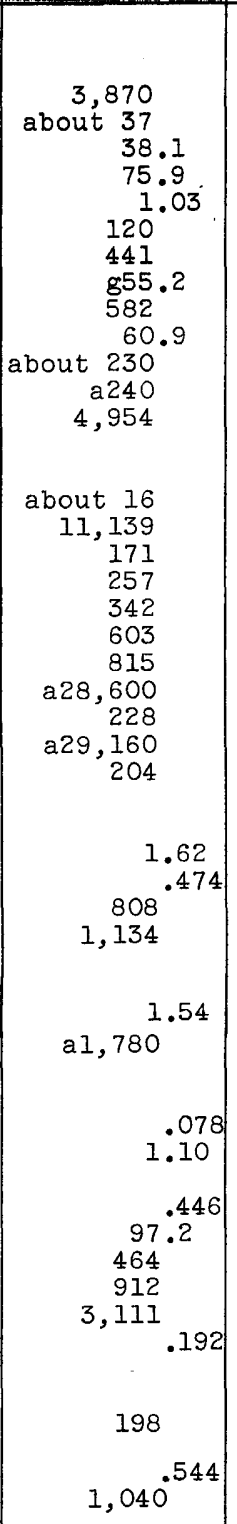 & 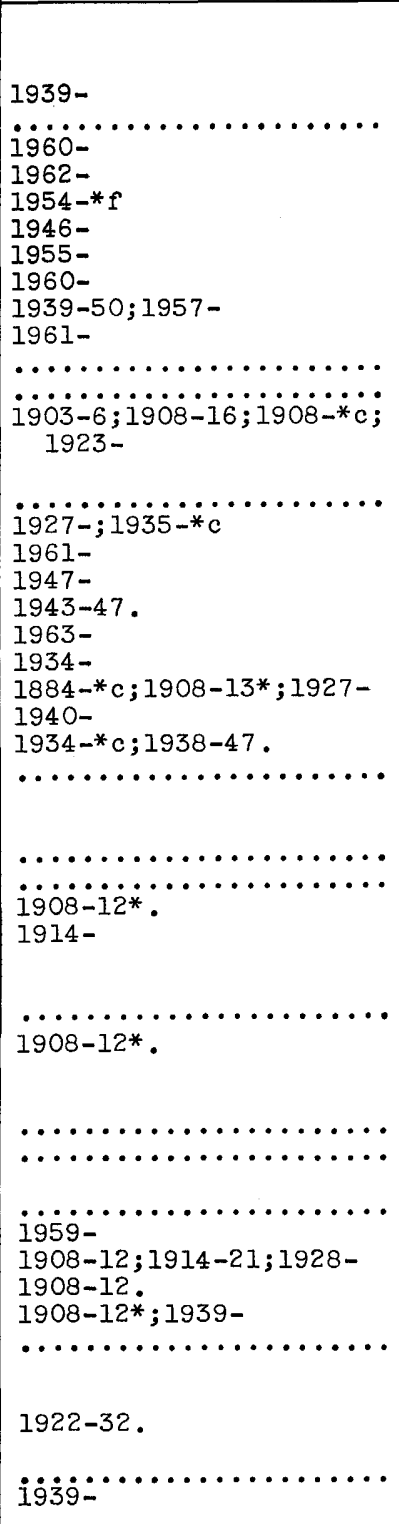 & $\begin{array}{l}1956- \\
1956- \\
1961- \\
1956- \\
1960- \\
1959-\end{array}$ & $\begin{array}{l}1954 ; 1960-62 . \\
1963- \\
1954 ; 1962-\end{array}$ \\
\hline
\end{tabular}


Black Branch:

Black Branch tributary near Junction, Ill...... Tradewater River basin

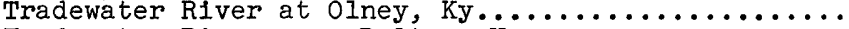

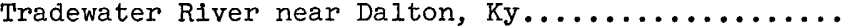
Clear Creek:

Weirs Creek:

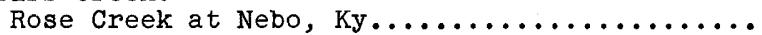

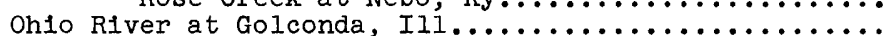
Bay Creek basin

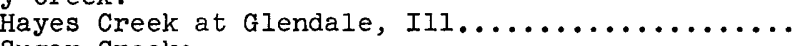

Lake Glendale Inlet near Dixon Springs, Ill....

Lake Glendale outlet near Dixon Springs, Ill.... Sugar Creek near Dixon Springs, Ill........... Cumberland River basin

Poor Fork (head of Cumberland River) at Harlan-Letcher County Iine, Ky.

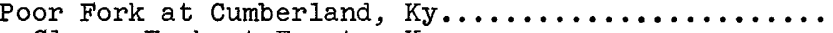

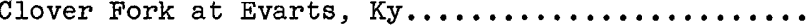

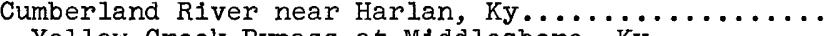

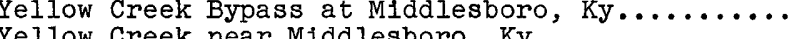

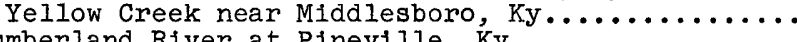

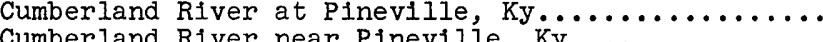

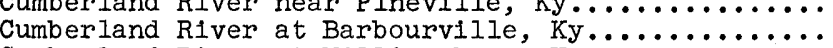

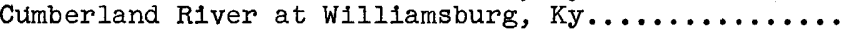

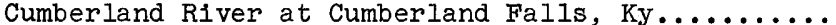

Laurel River:

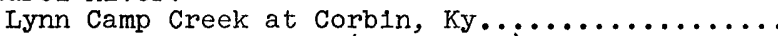

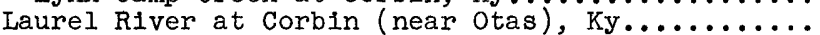

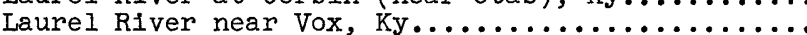

Rockcastle River:

Wood Creek near London, $\mathrm{Ky} \ldots \ldots \ldots \ldots \ldots \ldots \ldots$

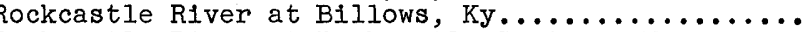

Rockcastle River at Rockcastle Springs, Ky.......

Hughes Fork Beaver Creek:

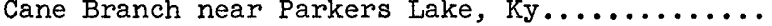

West Fork Cane Branch near Parkers Lake, Ky...

Beaver Creek:

Hurricane Fork:

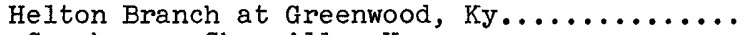

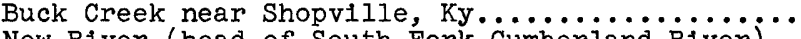

New River (head of South Fork Cumberland River)

$$
\text { near New River, Tenn. }
$$

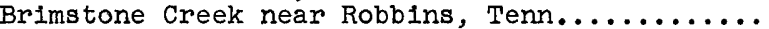

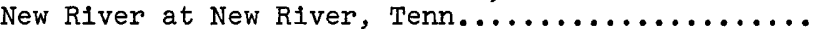
Clear Fork:

White Oak Creek at Sunbright, Tenn..........

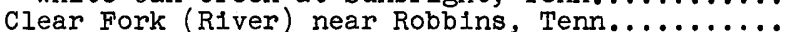
Black Creek:

Black Creek tributary near Robbins, Tenn...... 4096

*-Gage helghts, or gage heights and discharge measurements only.

a Approximately.

c In reports of U.S. Weather Bureau.

$f$ In files of Geological Survey.

8 Part of drainage area is noncontributing or does not contribute directly to surface runoff.
$1960-$

$1940-$

1928-31; 1937-40.

$1951-$

1924 -**; 1937 -

$1949-$

$1954-63 \ldots \ldots \ldots \ldots \ldots \ldots \ldots+1964-$

$1954-63$.

$1940-43$

$1940-$

$\ddot{194} \dot{0}$

i $\ddot{9} \dot{4} \dot{0}-$

1929-31.

$1938-$

\begin{tabular}{r|r}
960 & $1922-31 ; 1948-$ \\
1,607 & $1908-*$ c $1950-$
\end{tabular}

1,977

53.8
201

201
245

3.89

604

745

$\ldots$

1930-31.

$1953-$

$.671956-$

.26

$.85 \quad 1956-$

\begin{tabular}{l|l}
165 & $1952-$ \\
312 & $1922-34$
\end{tabular}

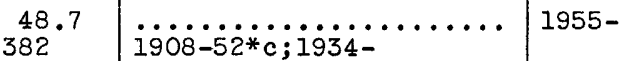

$13.5 \quad 1932-33 \ldots \ldots \ldots \ldots \ldots . \ldots 1955-$

.25

$1955-62$.

\section{$1954-55 ; 1959-$}

1940-41;1944-

$1956-$ 


\begin{tabular}{|c|c|c|c|c|c|}
\hline \multirow[b]{2}{*}{ Station name } & \multirow{2}{*}{$\begin{array}{r}\text { Station } \\
\text { number }\end{array}$} & \multirow{2}{*}{$\begin{array}{c}\text { Drainage area } \\
(\mathrm{sq} \mathrm{ml})\end{array}$} & \multicolumn{3}{|c|}{ Perlods of record } \\
\hline & & & $\begin{array}{c}\text { Daily or monthly } \\
\text { figures }\end{array}$ & Annual peaks & $\begin{array}{c}\text { Low-flow } \\
\text { measurements }\end{array}$ \\
\hline 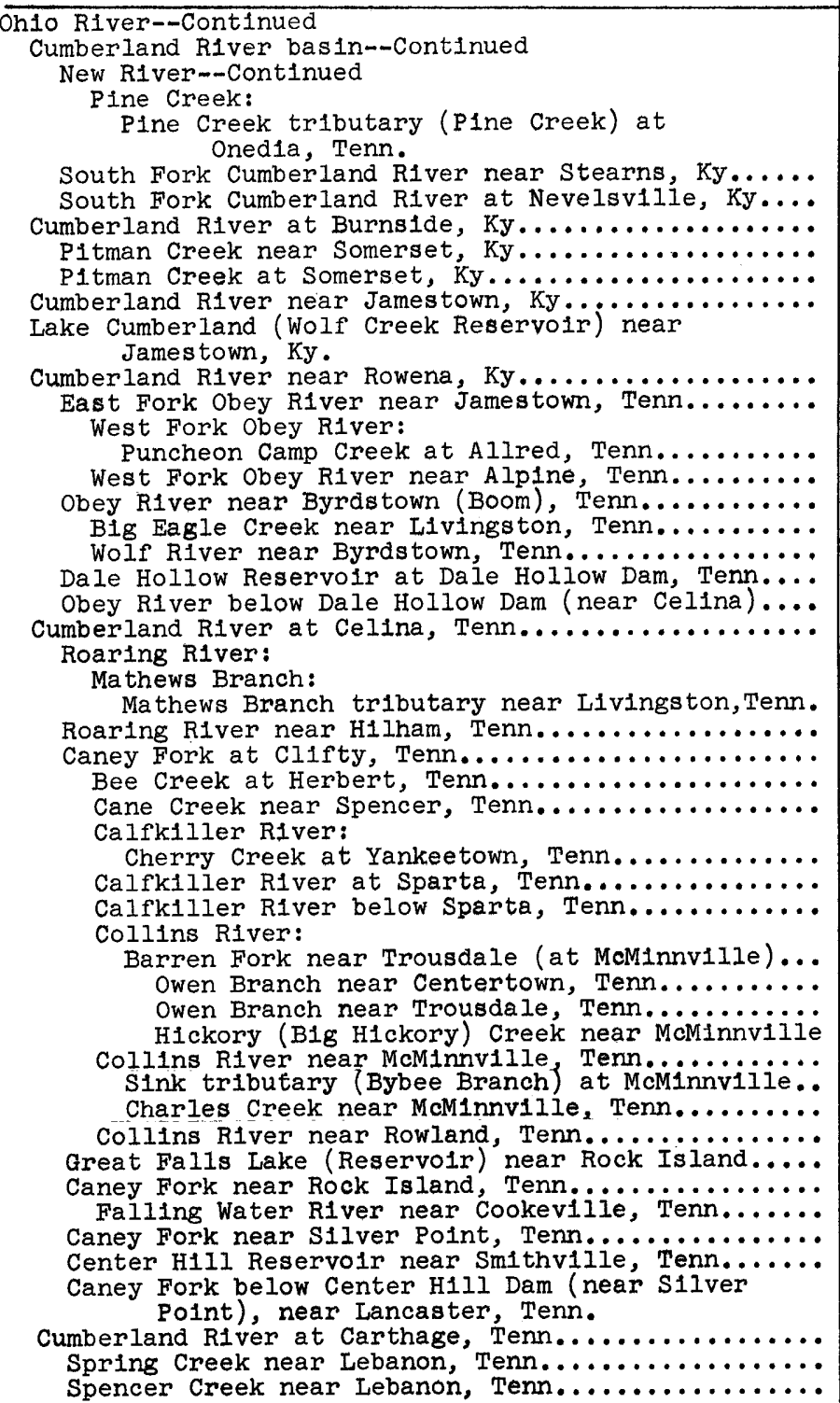 & $\begin{array}{l}4177 \\
4180 \\
4185 \\
4190 \\
4192 \\
4193 \\
4195 \\
4200 \\
\\
4205 \\
4206 \\
4207 \\
4208 \\
4210 \\
4211 \\
4212 \\
4215 \\
4220 \\
4225 \\
4230 \\
4235 \\
4240 \\
4245 \\
4250 \\
4255 \\
4257\end{array}$ & $\begin{array}{c}78.49 \\
111 \\
101 \\
134 \\
16.7 \\
157 \\
175 \\
126 \\
4.60 \\
9.42 \\
132 \\
642 \\
31.1 \\
755 \\
1,677 \\
1,678 \\
67.0 \\
\text { a2, } 100 \\
2,195 \\
\text { a2, } 200 \\
\text { a10, } 700 \\
35.3 \\
3.32\end{array}$ & 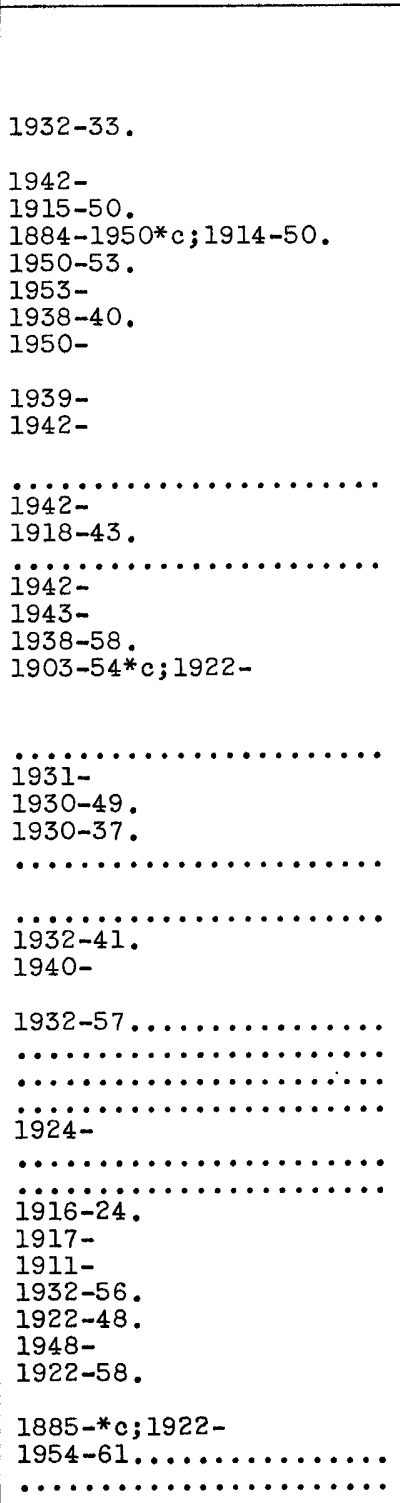 & $\begin{array}{l}1955- \\
\ldots \ldots \ldots \ldots \ldots \ldots \\
\ldots \ldots \ldots \ldots \ldots \\
1958- \\
1955- \\
1955-61 . \\
\ldots \ldots \ldots \ldots \ldots \\
1955-\ldots \ldots \ldots \ldots\end{array}$ & $\begin{array}{l}1930 ; 1951 ; 1959- \\
1959- \\
1950-55 ; 1959- \\
1951-55 ; 1959-\end{array}$ \\
\hline
\end{tabular}


Cedar Creek:

Cedar Creek tributary at Green H1ll, Tenn...... 4258

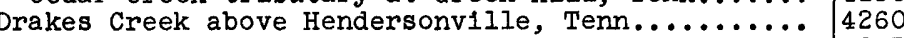

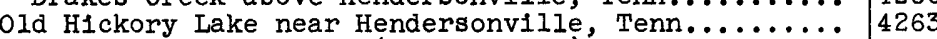
lumerland East Fork Stones River at Woodbury, Tenn.........

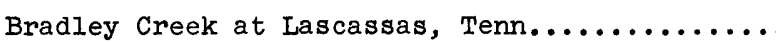
East Fork Stones River near Lascassas, Tenn....... West Fork Stones River near Murfreesboro, Tenn.

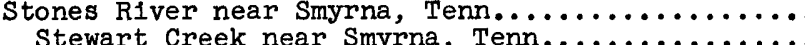

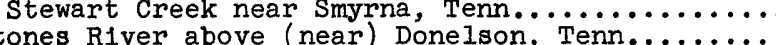

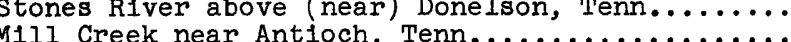

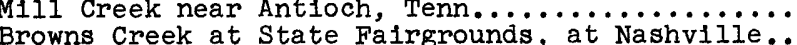

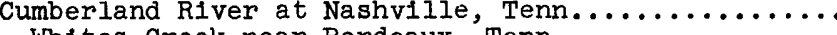
Whites Creek near Bordeaux, Tenn.

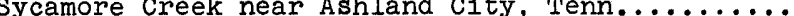
Harpeth River:

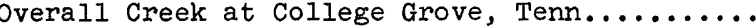

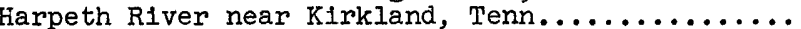

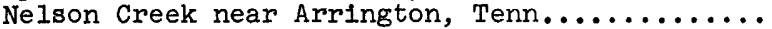

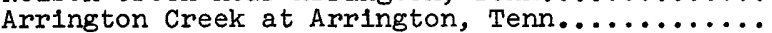

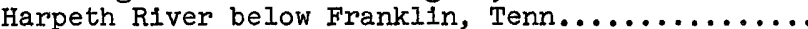
West Harpeth River near Leipers Fork, Tenn.....

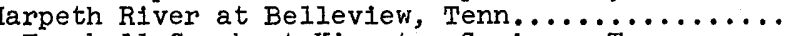
Iurnbull Creek at Kingston Springs, Tenn........

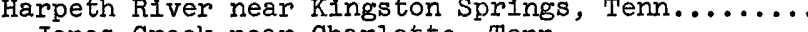

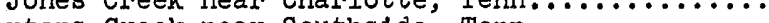

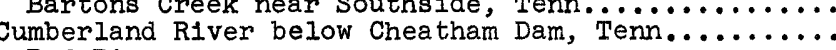
Red River:

Elk Fork near Sadersville, Tenn.............. 4354

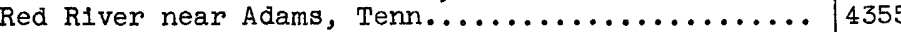

Sulphur Fork Red River:

Carr Creek near Springfield, Tenn.......... 4359

Sulphur Fork Red River near Adams, Tenn......... 4360

Red River at Port Royal, Tenn............... 4361

West Fork Red R1ver near New Providence, Tenn... 4362

Spring Creek near St. Bethlehem, Tenn......... 4363

Noahs Spring Branch at Fort Campbell, Ky -Tenn 4364

Cumberland River at Clarksville. Tenn............ 4365

Yellow Cheek near Shtloh, Tenn. . . . . ...............

Wellow Creek: near shilon,

Wells Creek:

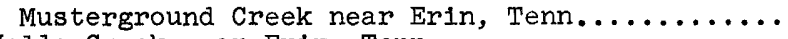

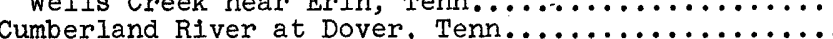

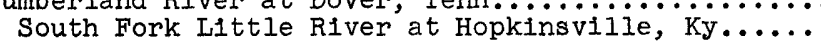

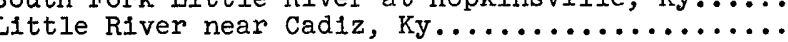

Cumberland River at Smithland, $\mathrm{Ky} \ldots \ldots \ldots \ldots \ldots \ldots$

Tennessee River basin

French Broad River (head of Tennessee River) at

East Fork French Broad River near Rosman, N.C....

* Gage helghts, or gage heights and discharge measurements only

Approximately.

In reports of U.S. Weather Bureau.

Part of drainage area is noncontributing or does not contribute directly to surface runoff.

Fragmentary.

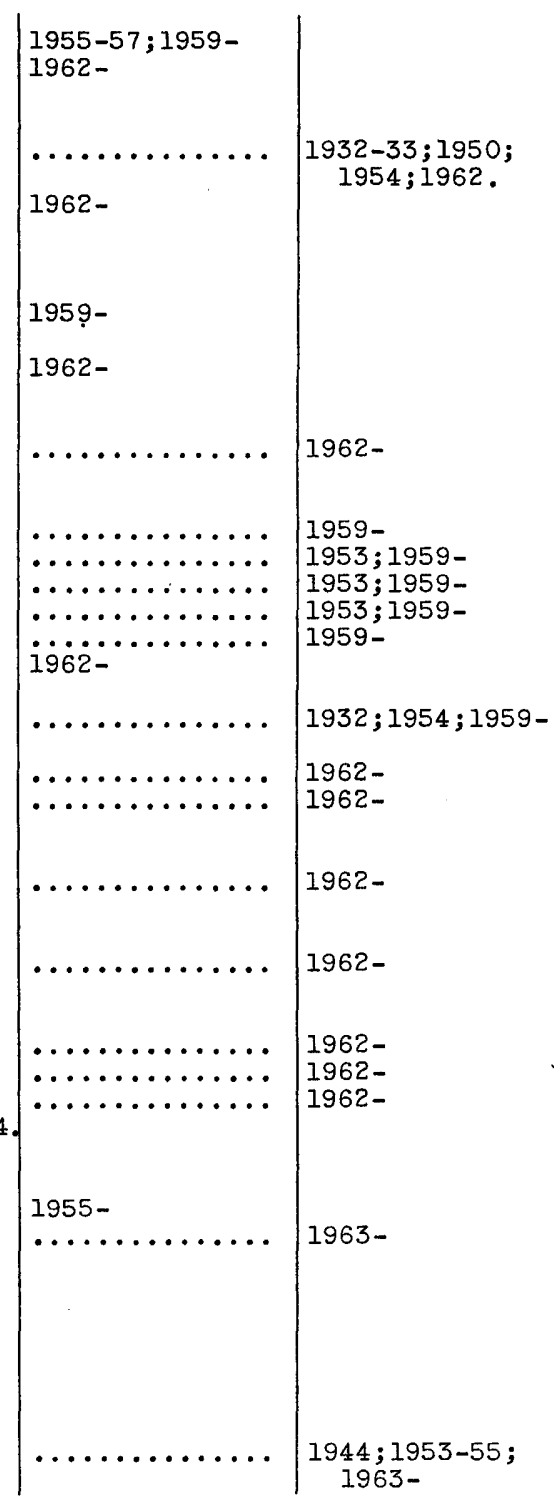




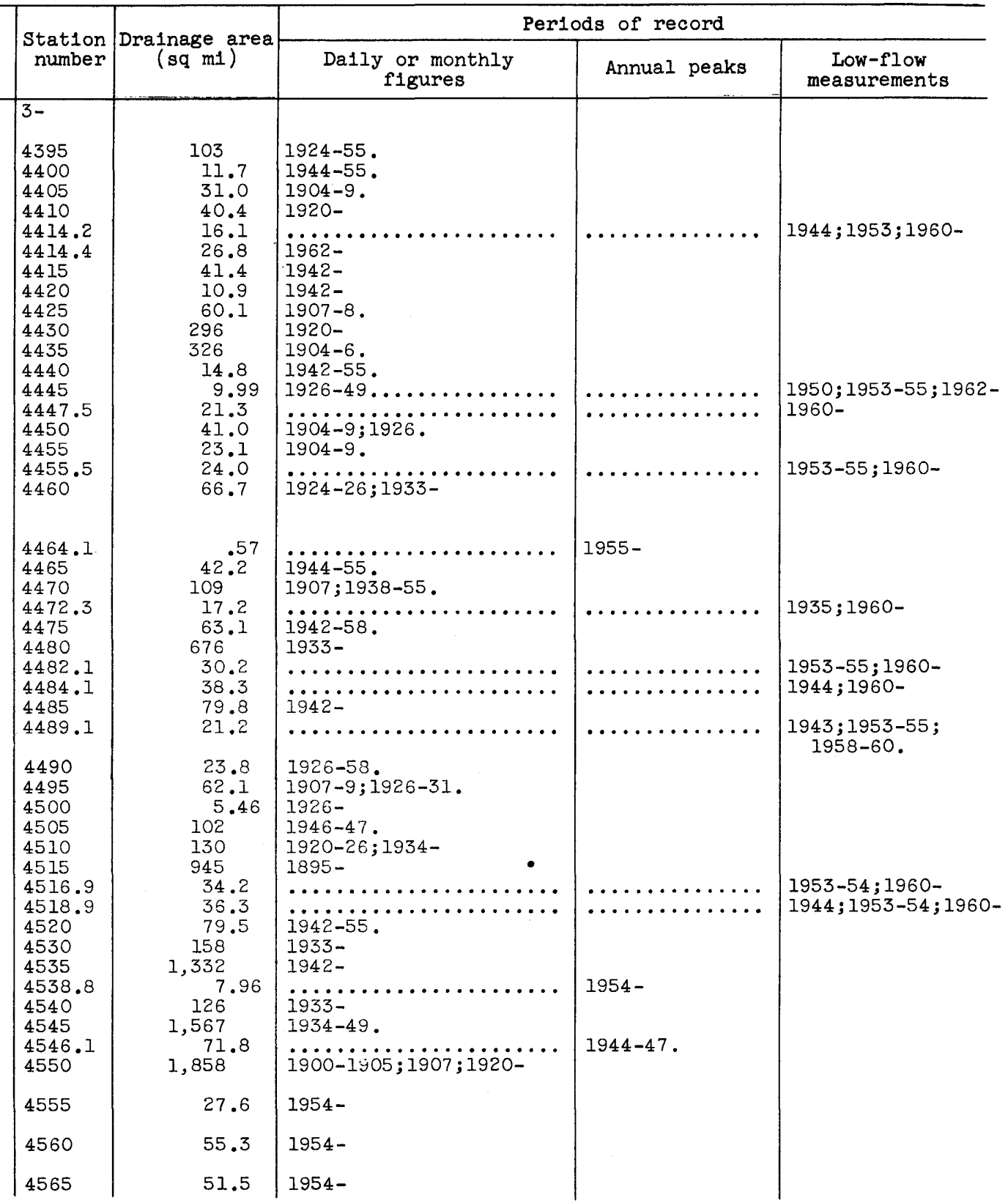

Oh10 R1ver--Cont1nued

Tennessee River basin--Continued

French Broad RIver at Calvert, N. C.

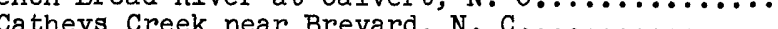

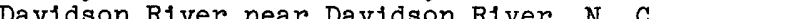

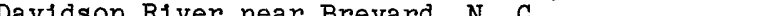

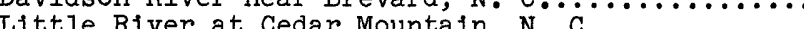

Ittle River above High

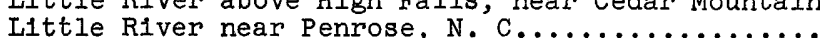

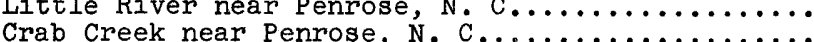

Ittle River at Calhoun, $N$.

Little River at Calhoun, N. C.....

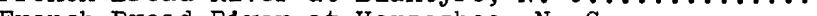

Boylston Rlver at Horseshoe, $N$.

Boylston creek near Horseshoe, N. C.............

South Fork Mills River at The Plink Beds, N. C......

South Fork Mills River near Pisgah Forest, N. C...

South Fork Milis River near Sitton, N. C.......

North Fork Mills River near Milis River, N. C...

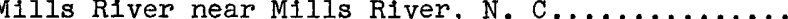
Mud Creek:

Clear Creek:

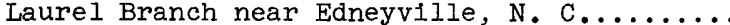

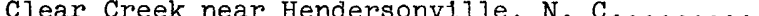

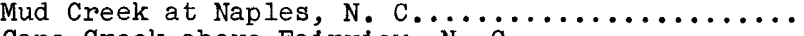

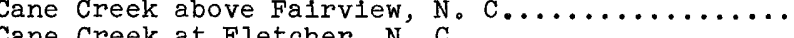

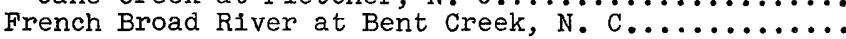

Hominy Creek above South Hominy Creek, at Candler.

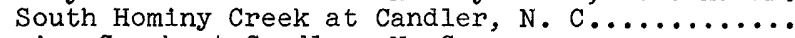
Hominy Creek at Candler, $N$. C...................

North Fork Swannanoa River near Black Mounta1n..

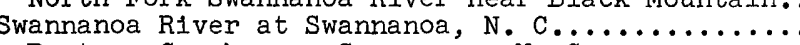

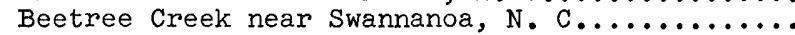

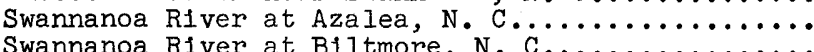
Swannanoa River at Biltmore, $\mathrm{N}^{\mathrm{C}} \mathrm{C} \ldots \ldots \ldots \ldots \ldots \ldots$

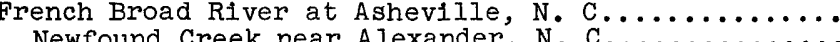
Newfound Creek near Alexander, $N$. C . . . . . . . . .

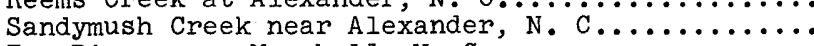
Ivy R1ver near Marshall, $\mathrm{N}^{\mathrm{C}} \ldots \ldots \ldots \ldots \ldots \ldots \ldots$

French Broad River at Marshail, N.

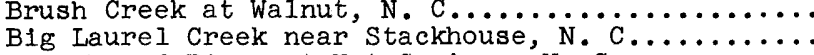

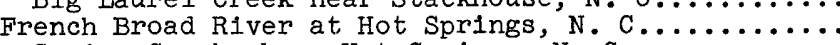

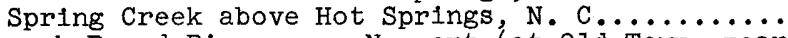

French Broad River near Newport (at old Town, near Newport), Tenn.

West Fork Pigeon River above Lake Logan, near Hazelwood, N. C.

West Fork P1geon River below Lake Logan, near Waynesville, N. C.

East Fork Pigeon River near Canton, N. C....... 4565 


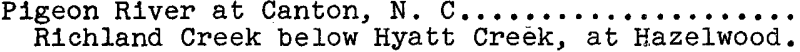

Allen Creek near Hazelwood, N. Richland Creek at waynesville, Pigeon River near Crabtree, N. C

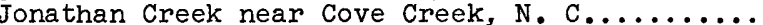

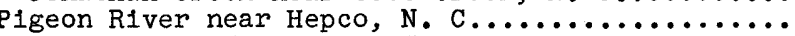

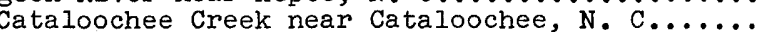

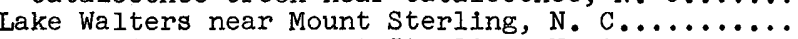

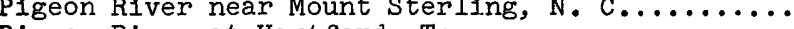

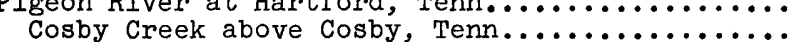

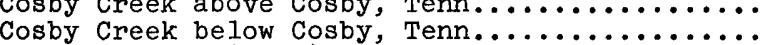

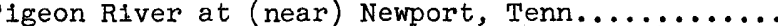
North Toe River (head of Nolichucky River), at Newland, N. C.

North Toe River at Altapass (above Spruce Pine)...

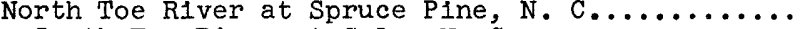

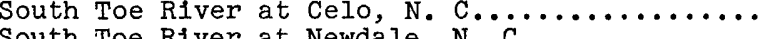
B1g Rock Creek near Green Mountain, N. C........... Cane River:

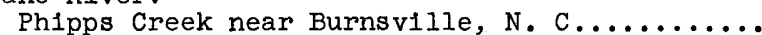

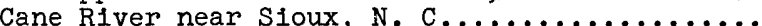

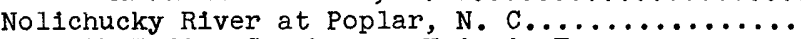

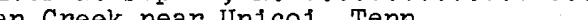
Nollchucky River at (near Chucky Valley)

$$
\text { Embreeville, Tenn. }
$$

Big Limestone Creek:

Muddy Fork near Falrview, Tenn

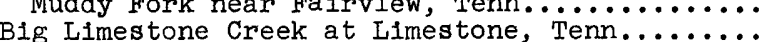
Nolichucky RIver below Nolichucky Dam (near Greenville), Tenn.

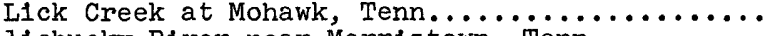

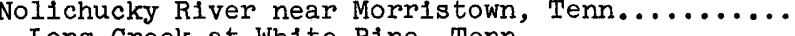

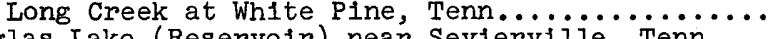
Douglas Lake (Reservolr) near Seviervilie, Tenn.... Little Pigeon River near Pittman Center, Tenn..... Little Pigeon River near Sevierville, Tenn........ East Fork Little Pigeon River near' Seviervilie.

Little Pigeon River above West Prong, at Sevierville, Tenn.

West Fork Little Pigeon River

Hog Pen Branch near Gatlinburg, Tenn......... West Fork Little Pigeon River near Pigeon Forge.

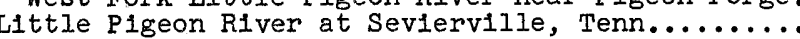

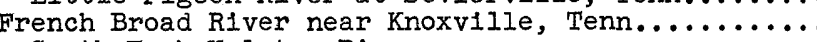
South Fork Holston River:

Dickey Creek:

Steve Keesling Spring at Sugar Grove, Va..... South Fork Holston River at Riverside, near Chilhowie (near Chilhowie), Va.

South Fork Holston River near Chilhowie, Va....... 4720

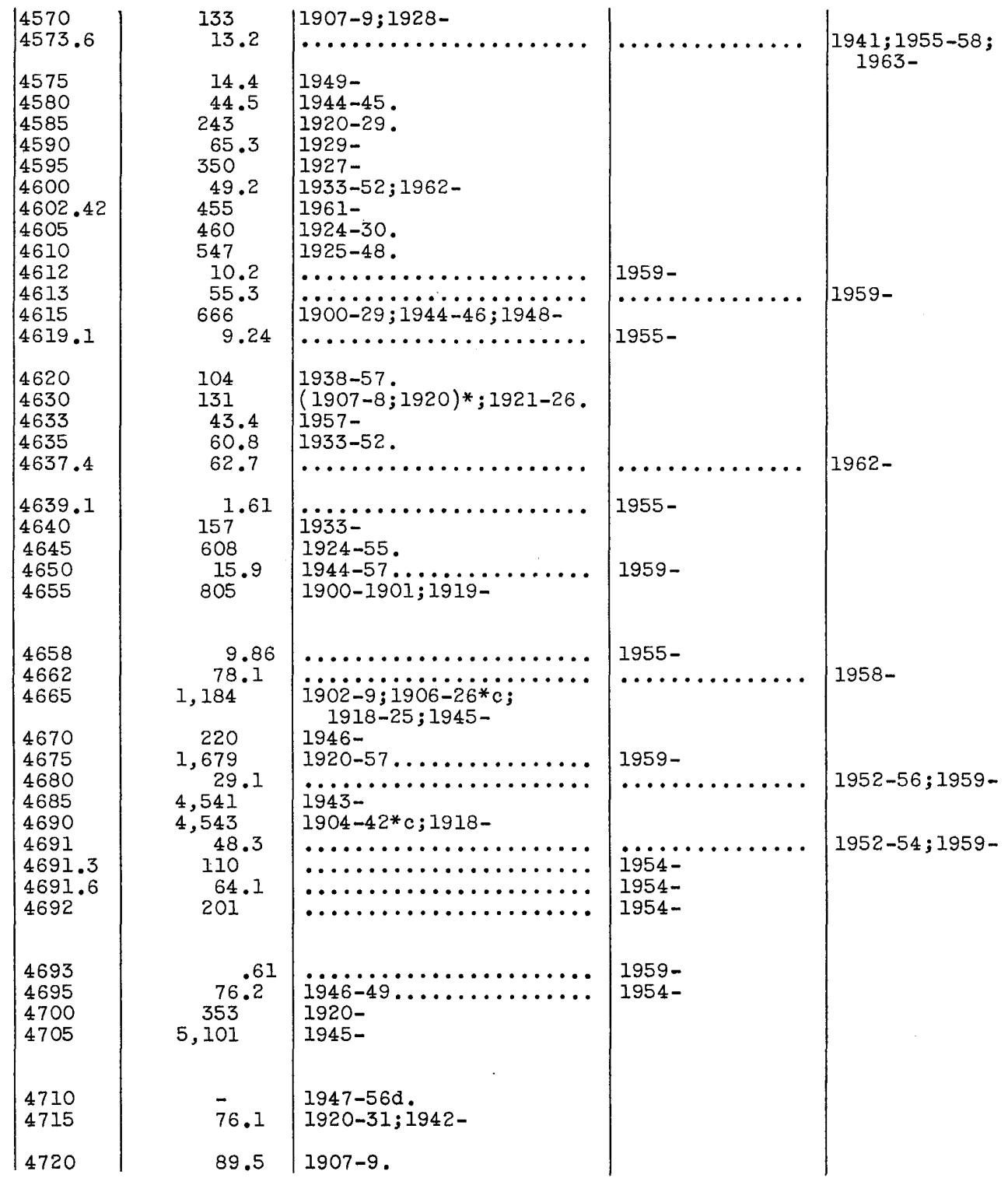

* Gage helghts, or gage helghts and discharge measurements only.

c In reports of U.S. Weather Bureau.

d Discharge measurements only. 


\begin{tabular}{|c|c|c|c|c|c|}
\hline \multirow[b]{2}{*}{ Station name } & \multirow{2}{*}{$\begin{array}{r}\text { Stat1on } \\
\text { number }\end{array}$} & \multirow{2}{*}{$\begin{array}{c}\text { Drainage area } \\
(\mathrm{sq} \mathrm{ml})\end{array}$} & \multicolumn{3}{|c|}{ Periods of record } \\
\hline & & & $\begin{array}{c}\text { Daily or monthly } \\
\text { figures }\end{array}$ & Annual peaks & $\begin{array}{c}\text { Low-flow } \\
\text { measurements }\end{array}$ \\
\hline 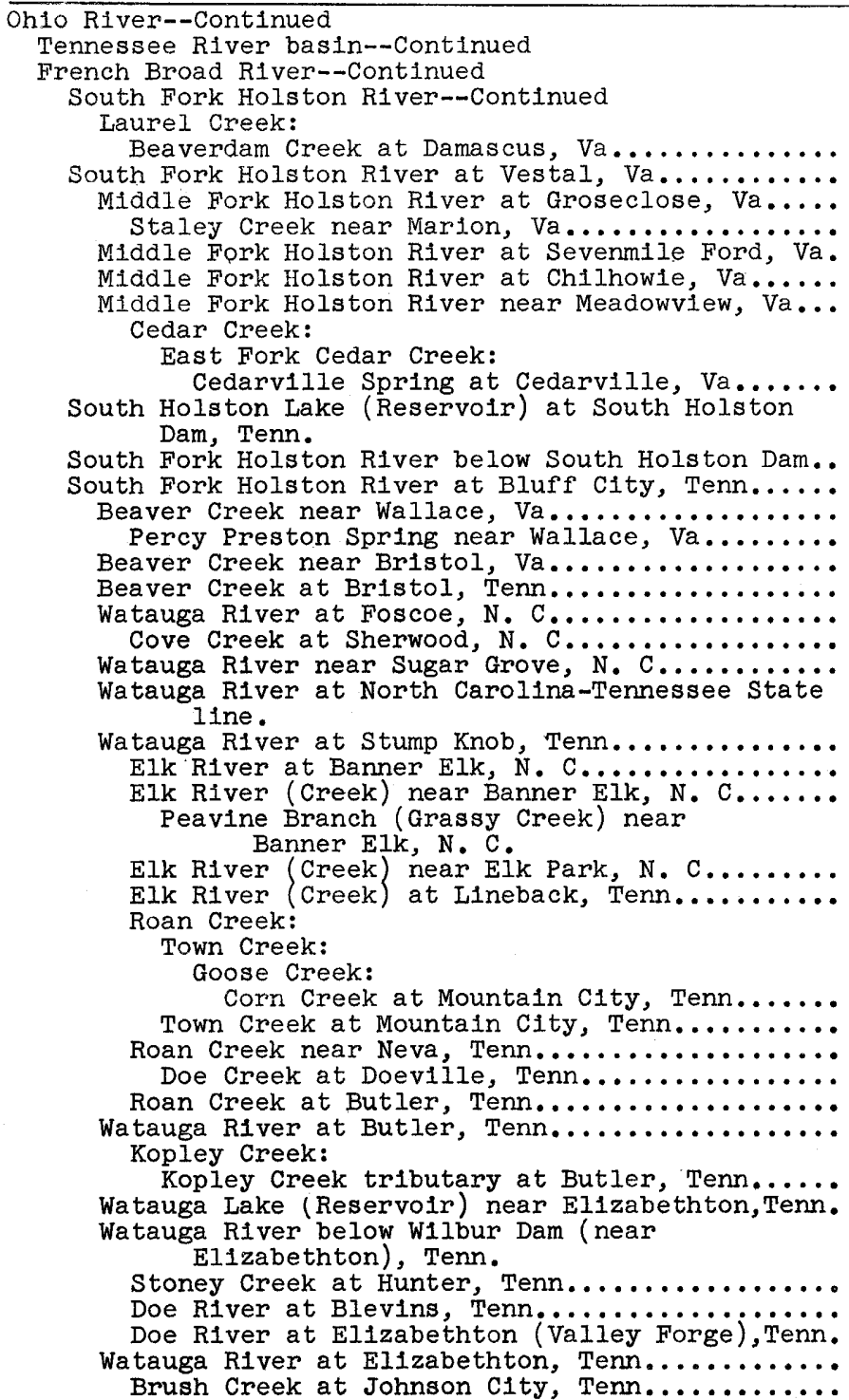 & $\begin{array}{l}4725 \\
4730 \\
4735 \\
4738 \\
4740 \\
4745 \\
4750 \\
\\
\\
4755 \\
4760 \\
\\
4765 \\
4770 \\
4775 \\
4780 \\
4784 \\
4785 \\
4787.2 \\
4789.1 \\
4790 \\
4795 \\
4800 \\
4804.1 \\
4805 \\
4805.4 \\
4810 \\
4815\end{array}$ & $\begin{array}{l}56.0 \\
301 \\
7.39 \\
8.33 \\
132 \\
155 \\
211 \\
\\
- \\
703 \\
703 \\
813 \\
13.7 \\
-77.7 \\
44.8 \\
10.6 \\
23.1 \\
90.8 \\
152 \\
171 \\
7.44 \\
17.8 \\
.51 \\
42.0 \\
82.0\end{array}$ & 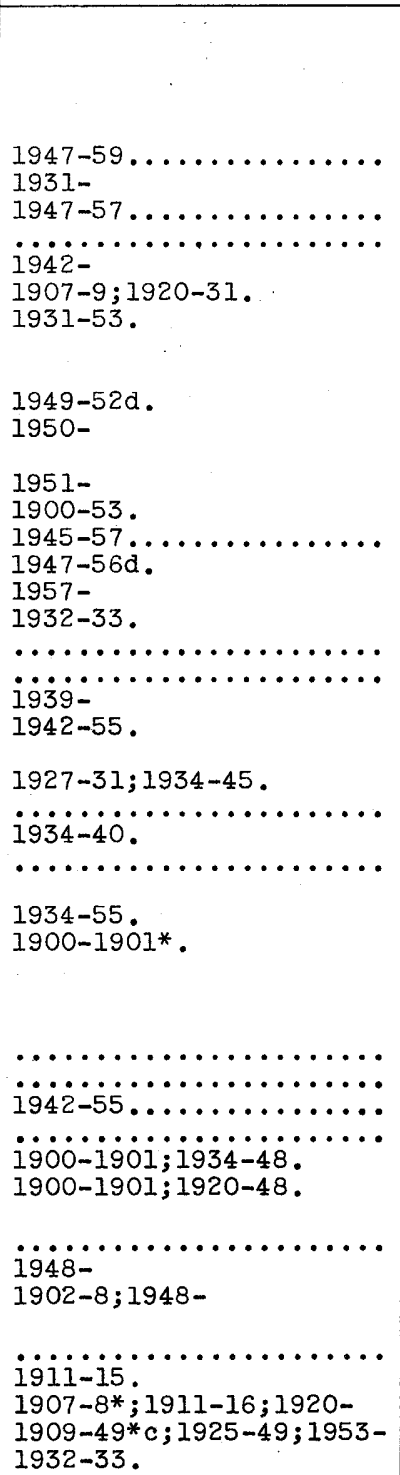 & $\begin{array}{l}1959-61 ; 1963- \\
190 \ldots \ldots \ldots \\
\cdots \ldots \ldots \ldots \ldots \ldots\end{array}$ & $\begin{array}{l}1952-56 ; 1959- \\
1952-56 ; 1959-\end{array}$ \\
\hline
\end{tabular}


Boone Lake (Reservoir) at Boone Dam, Tenn........ 4868 Fort Patrick Henry Lake (Reservoir) at Fort Patrick Henry Dam, Tenn.

South Fork Holston River at Kingsport, Tenn.......

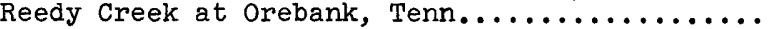

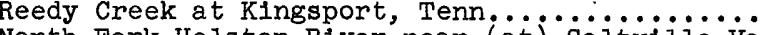
North Fork Holston River near (at) North Fork Holston River near Plasterco, Va..... Worth Fork Holston River at Holston, Va..........

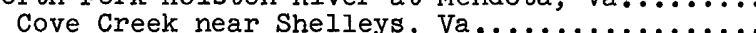

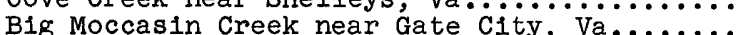
orth Fork Holston River near Gate City, Va......

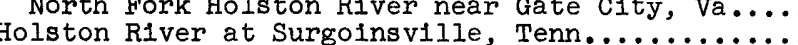

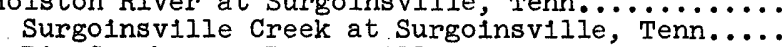

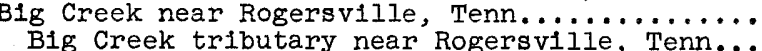
Big Creek tributary near Rogersvilie, Tenn...

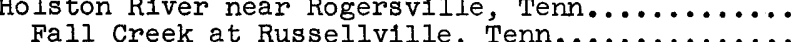

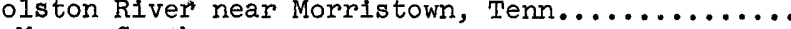
Mossy Creek:

Mossy Creek tributary:

Mossy Spring near Jefferson City, Tenn......

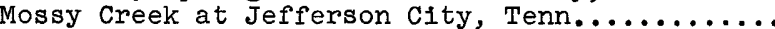
Cherokee Lake (Reservoir) near Jefferson City..... Holston River near Jefferson City, Tenn............ M1ll Spring Creek:

M111 Spring near Jefferson C1ty, Tenn.........

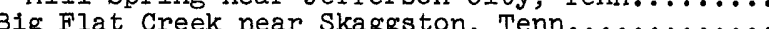
Holston River near Knoxville (at Strawberry Plains), Tenn.

Tennessee River:

First Creek at Mineral Springs Avenue, at. Knoxville, Tenn

First Creek at Fifth Avenue, at Knoxville (at Knoxvilie), Tenn.

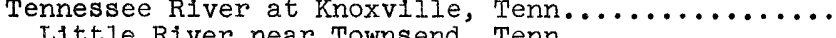

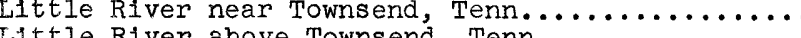

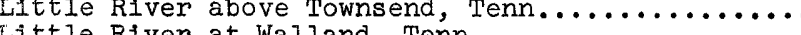

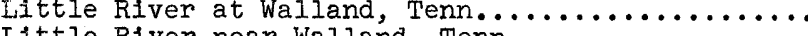

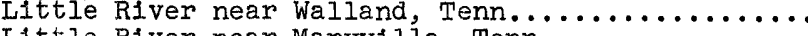

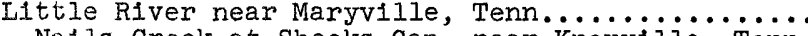
Na1ls Creek at Shooks Gap, near Knoxvilie, Tenn.

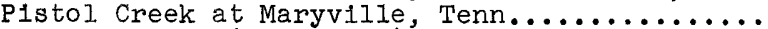
Fort Loudoun Lake (Reservoir) near Lenoir City,Tenn. Tennessee River:

Little Tennessee River near Prentiss, N. C.......

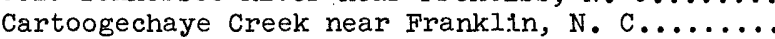

Cullasaja River (Creek) at Highlands, N. C..... Cullasaja River (Creek) at Cullasaja, N. C...... Little Tennessee River at Franklin, N. C.........

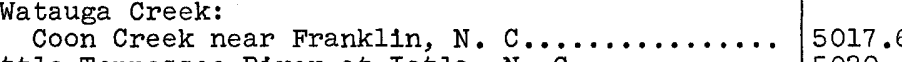

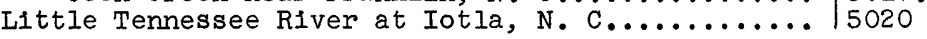

* Gage helghts, or gage heights and discharge measurements only. c In reports of U.S. Weather Bureau.

d Discharge measurements only.

$f$ In files of Geological Survey.

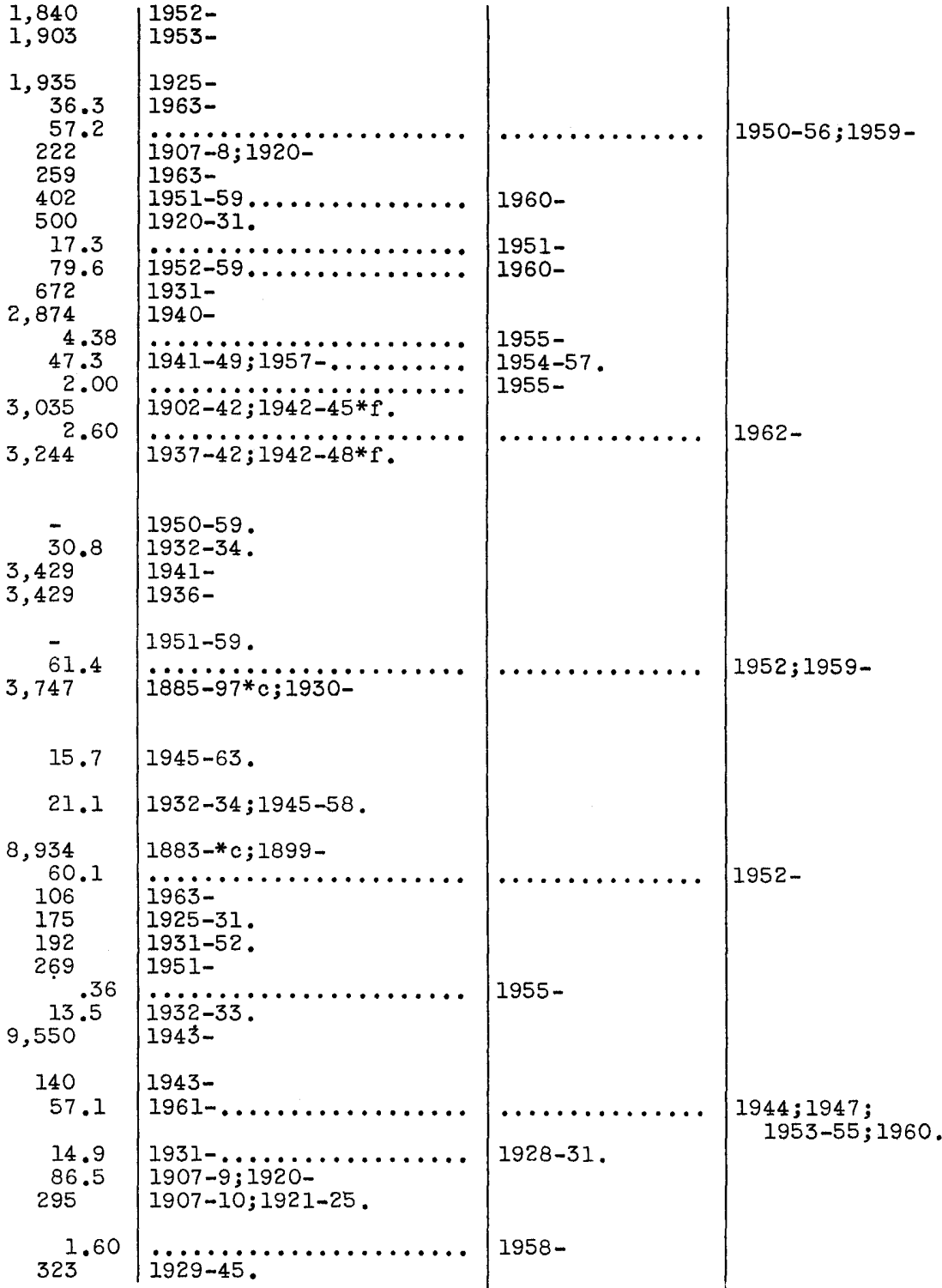

1,840 4875 


\begin{tabular}{|c|c|c|c|c|c|}
\hline \multirow[b]{2}{*}{ Station name } & \multirow{2}{*}{$\begin{array}{r}\text { Station } \\
\text { number }\end{array}$} & \multirow{2}{*}{$\begin{array}{c}\text { Drainage area } \\
(\mathrm{sq} \mathrm{m} 1)^{-}\end{array}$} & \multicolumn{3}{|c|}{ Periods of record } \\
\hline & & & $\begin{array}{c}\text { Da1ly or monthly } \\
\text { figures }\end{array}$ & Annual peaks & $\begin{array}{l}\text { Low-flow } \\
\text { measurements }\end{array}$ \\
\hline 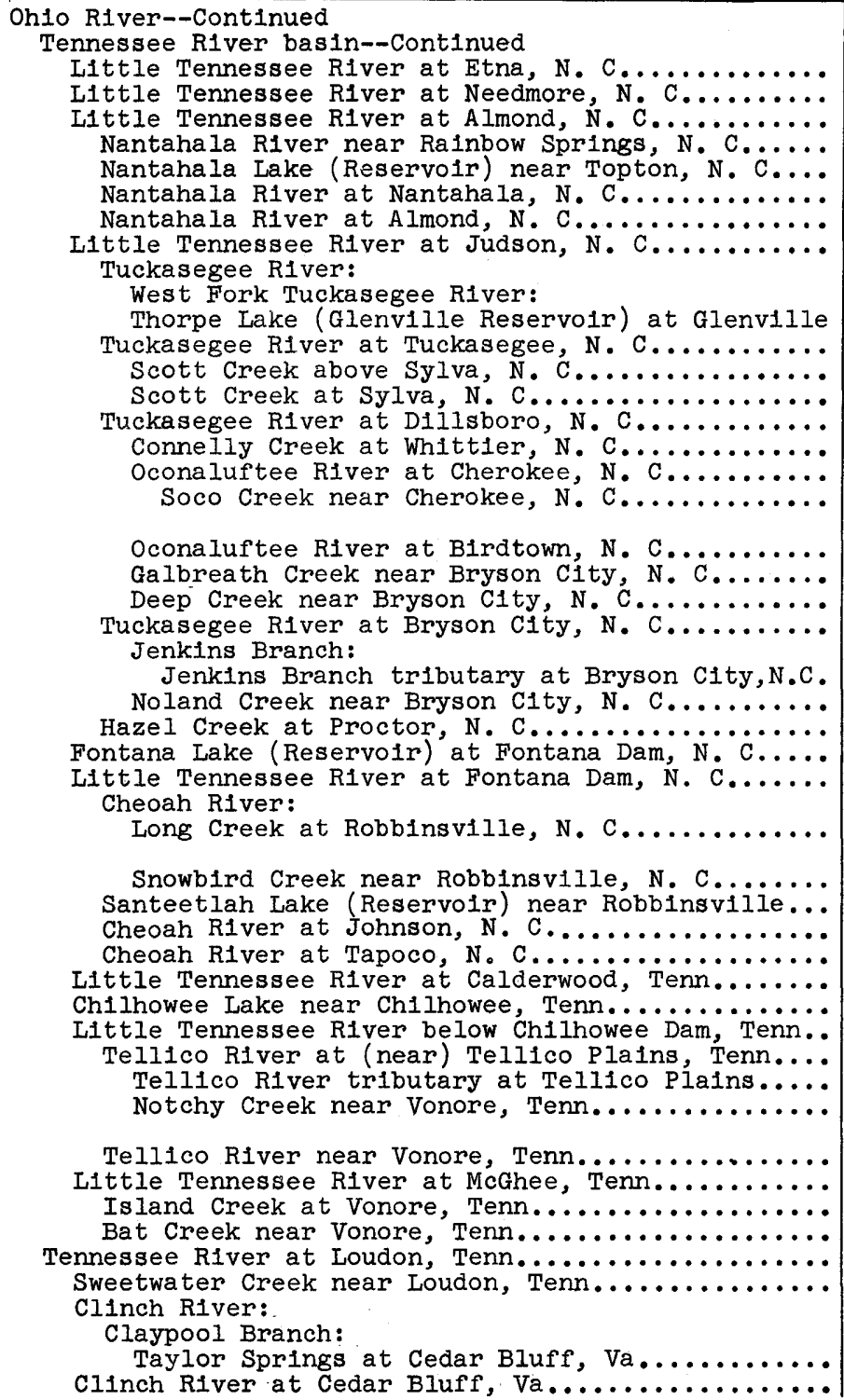 & \begin{tabular}{|l|}
$3-$ \\
5025 \\
5030 \\
5035 \\
5040 \\
5045 \\
5055 \\
5065 \\
5070 \\
\\
5075 \\
5080 \\
5090 \\
5095 \\
5105 \\
5106.1 \\
5110 \\
5113.6 \\
5120 \\
5123.6 \\
5127.4 \\
5130 \\
5134.1 \\
5135 \\
5140 \\
5145 \\
5150 \\
5156.1 \\
5160 \\
5165 \\
5170 \\
5175 \\
5180 \\
5182 \\
5183 \\
5185 \\
5186 \\
5188 \\
5190 \\
5195 \\
5196 \\
5197 \\
5200 \\
5201 \\
\\
5205 \\
5210 \\
\end{tabular} & 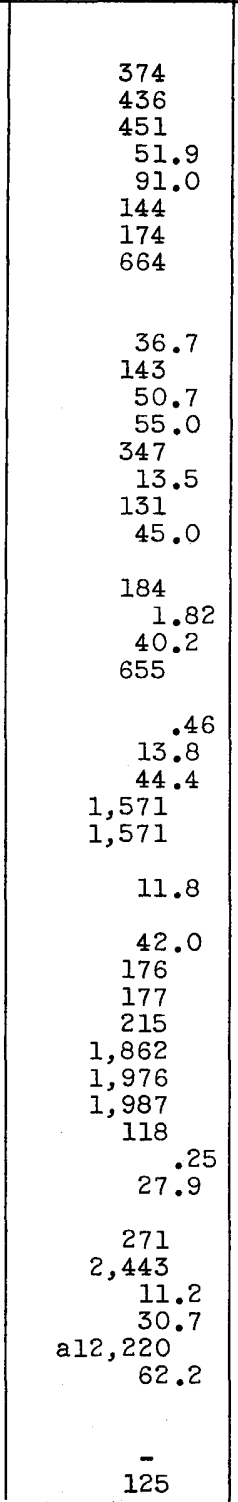 & 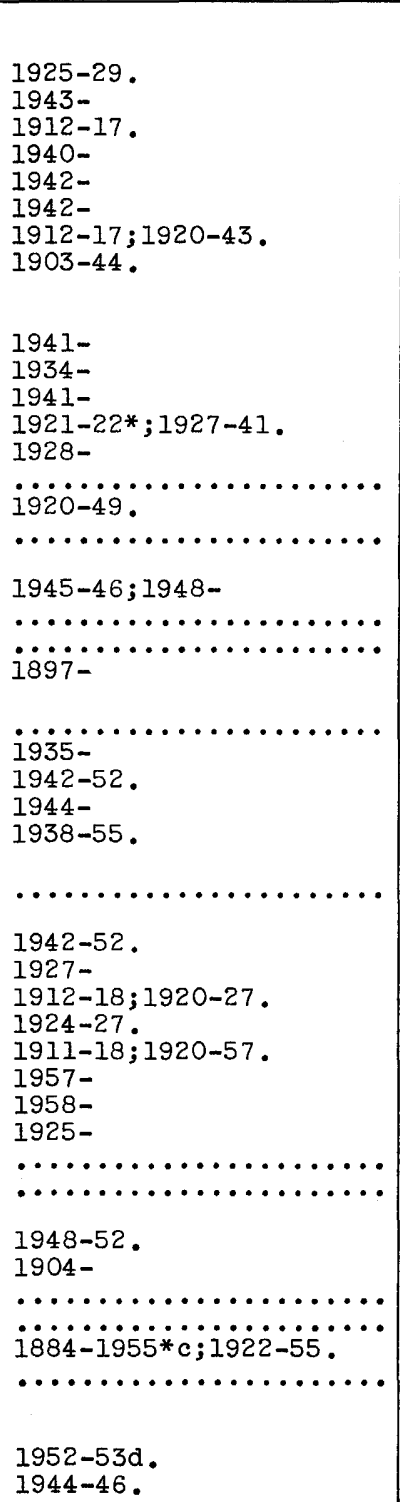 & $\begin{array}{l}\ldots \ldots \ldots \ldots \ldots \\
\ldots \ldots \ldots \ldots \ldots \ldots \\
\ldots \ldots \ldots \ldots \ldots \ldots \ldots \\
1955-\end{array}$ & $\begin{array}{l}1953-58 ; 1961-62 . \\
1925 ; 1944 ; 1947 ; \\
1953-54 ; 1958-63 . \\
1952-55 ; 1961-64 . \\
1944 ; 1953-\end{array}$ \\
\hline
\end{tabular}




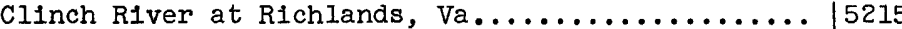

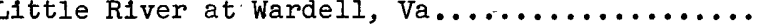

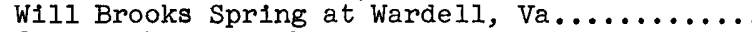

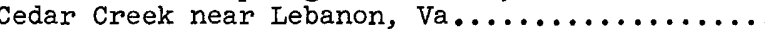

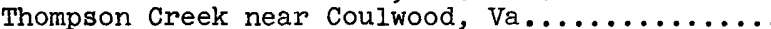

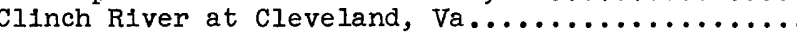
Guest River at Coeburn, va. $\ldots \ldots \ldots \ldots \ldots \ldots \ldots \ldots . . . . . . . .$.

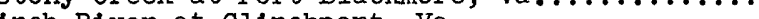

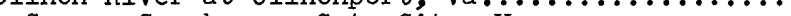

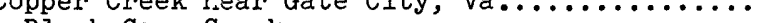
Plank Camp Creek:

$$
\text { Alley Valley Creek: }
$$

Quillin Spring near Gate City, Va........ 5265

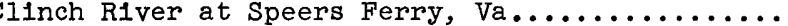

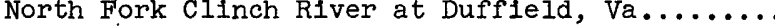

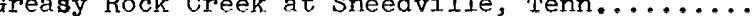
Clinch River above (near) Tazewell (near Lone Mountain), Tenn.

Big Sycamore Creek near Springdale, Tenn....... 528

Powell River at Big Stone Gap, Va............ 5295

South Fork Powell River at Big Stone Gap, Va.. 5300

North Fork Powell River at Pennington Gap, Va. 5305

Powell River near Pennington Gap, Va......... 5310

Powel1 River near Jonesville Va.......................... 5315

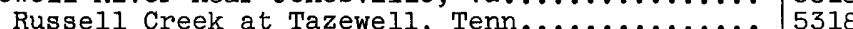

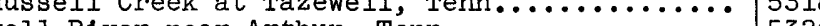

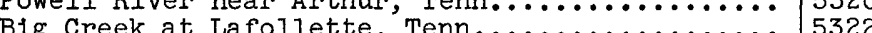

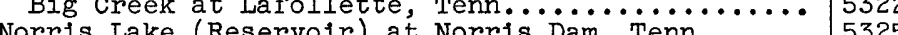

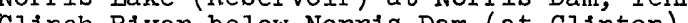

Clinch River below Norris Dam (at Clinton)

(near Coal Creek), Tenn.

Coal Creek at Lake City (Coal Creek), Tenn.....

Hinds Creek:

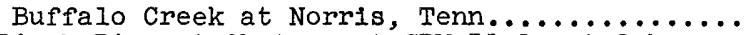

Clinch River tributary at CRM 5i.i, at oak Ridge, Tenn

Emory Valley Creek at Oak Ridge, Tenn.......... Bullrun Creek near Halls Crossroads, Tenn......

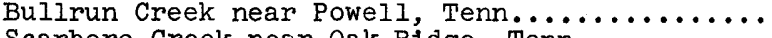

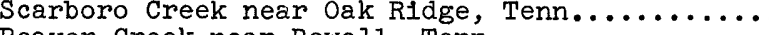

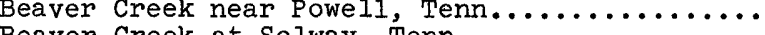

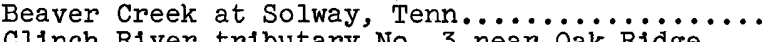
Clinch River tributary No 3 near

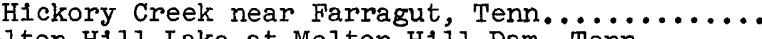

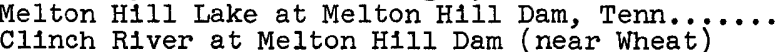

$$
\text { (near Scarboro), Tenn. }
$$

Whiteoak Creek above Oak Ridge Nat1onal Laboratory, near Oak Ridge, Tenn. Whiteoak Creek at Oak Ridge National Iaboratory, near Oak Rldge, Tenn.

Whiteoak Creek below Oak Ridge National Laboratory, near Oak RIdge, Tenn.

Melton Branch near Oak Rldge, Tenn........... Whiteoak Creek at Whlteoak Dam, near dak Ridge.

* Gage helghts, or gage helghts and discharge measurements only a Aproximately.

c In reports of U.S. Weather Bureau.

d Discharge measurements only.

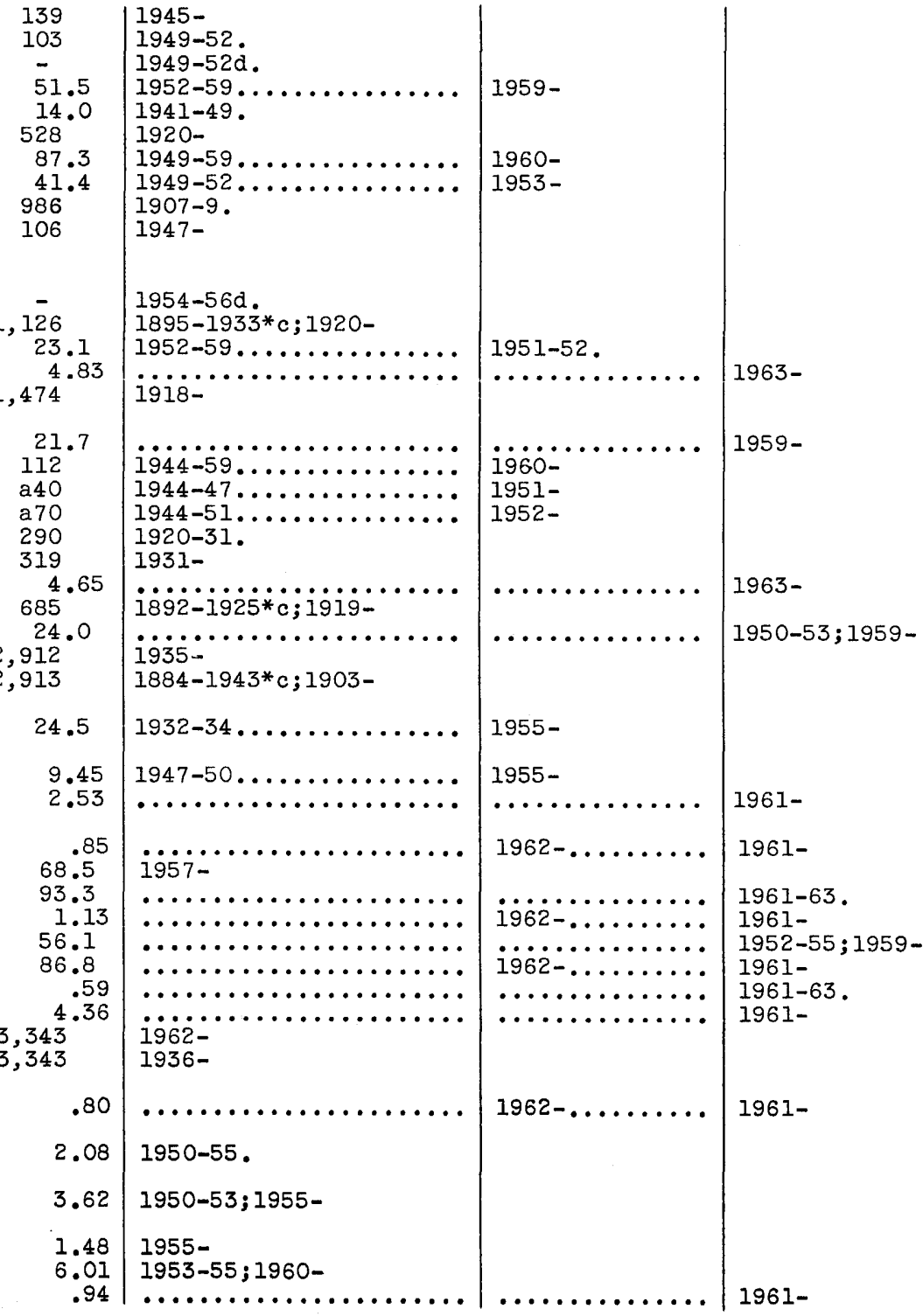

$1961-$ 


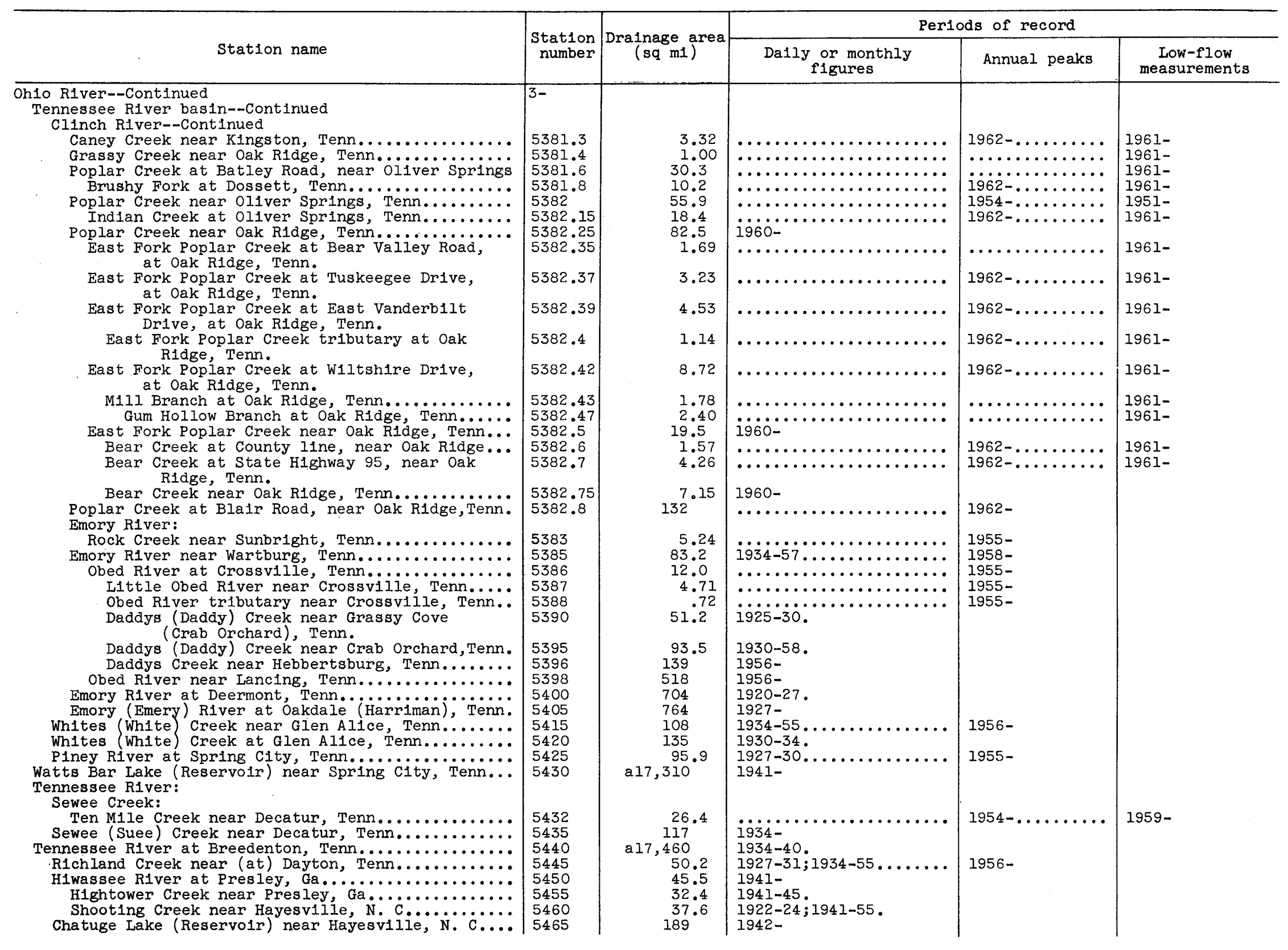


Hiwassee River below Chatuge Dam, near Hayesville (near Hayesville), N. C.

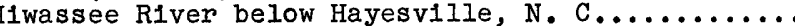

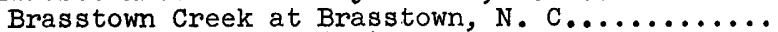

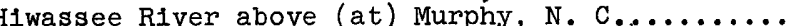

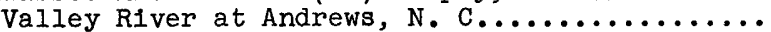

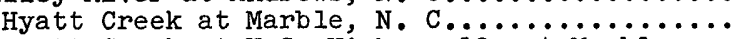
Hyatt Creek at U.S. Highway 19, at Marble....

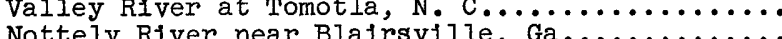

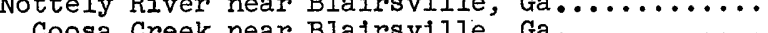

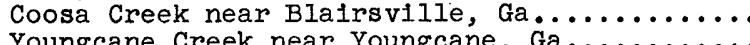

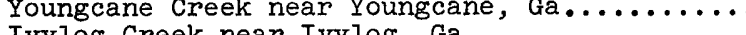

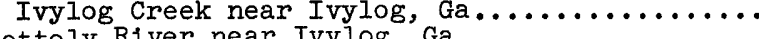

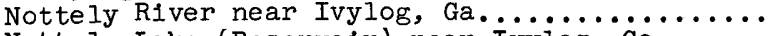
Nottely Lake (Reservolr) near Ivylog, $\mathrm{Ga} . . . . .$. Nottely River at Nottely Dam, near Ivylog, Ga...

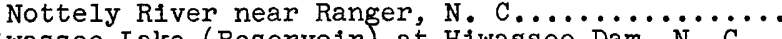
Hiwassee Lake (Reservoir) at Hiwassee Dam, N. C.. iwassee River at Hiwassee Dam (near Vests), $N$. palachia Lake (Reservolr) at Apa lachia Dam, N. C. Hiwassee River:

Turtletown Creek at Turtletown, Tenn..........

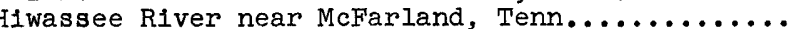

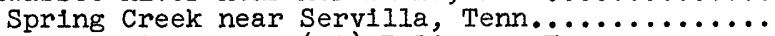

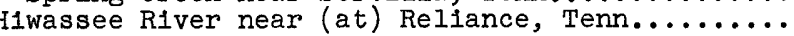

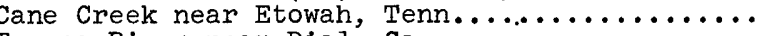
Blue Ridge Lake (Reservolr) near Biue Ridge, Ga Toccoa River near Blue Ridge (Morganton), Ga...

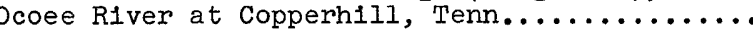
Flghtingtown Creek at McCaysvilie, Ga......... Davis Mill (Mill) Creek at Copperhili; Tenn... North Potato (Potato) Creek near Ducktown....

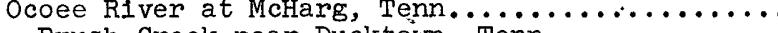

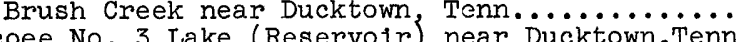
Ocoee No. 3 Lake (Reservoir) near Ducktown, Ienn

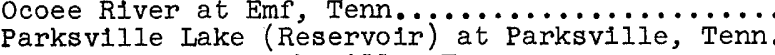

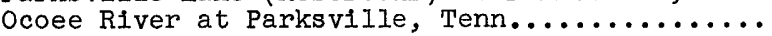

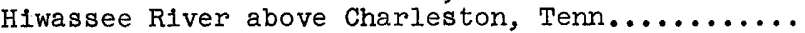

South Chestuee Creek near Benton, Tenn.........

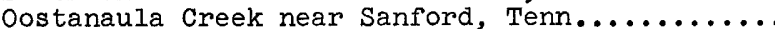

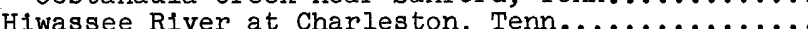

South Mouse Creek at Cleveland, Tenn........... South Mouse Creek tributary near cleveland... Rogers Creek near Lamontville, Tenn.......... Candies Creek:

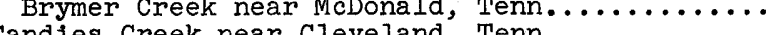

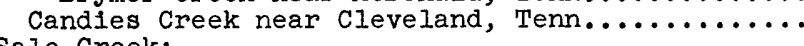
Sale Creek:

Rock Creek at Sale Creek, Tenn.

5470
5480
5483.3
5485
5495
5498.1
5498.2
5500
5505
5510
5515
5520
5525
5530
5535
5540
5545
5550
5552.1
5555
5560
5565
5567
5570
5572
5580
5585
5590
5595
5600
5605
5610
5615
5620
5625
5630
5640
5645
5650
5653
5655
5660

5660.5
5661
5661.5
5662
5662.5
5663
575

* Gage helghts, or gage heights and discharge measurements only.

Approximately.

c In reports of U.S. Weather Bureau.

$\mathrm{k}$ In U.S. War Department Stages of Ohio River and Principal Tributaries, Part 1.
$1944 ; 1953-55 ; 1958-$

$1955-$

$1944 ; 1951-55 ; 1961$

$1959-$

$1,136.82$

1,223

10.3

177

233

352

70.9
5.16

13.0

447

14.4
496

496
524

595

595

, 001.8

57.0

2,298

15.6

1.31
44.5

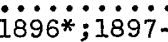

$1946-47$.

$\ldots \ldots \ldots \ldots \ldots \ldots \ldots$

1904-9;ioiu-17;iois-

1942

$1942-45$.

$1941-45$.

$1941-45$.

$1936-42$

$1942-$

1942-

$1901-5$; 1914-17; 1918-45.

$1934-43$

ïis̈-.

$1955-$

$1933-$

$1942-$

$\ddot{1} \ddot{0} \ddot{1} \ddot{i} \ddot{8} \ldots \ldots \ldots$.

19; $1918-48$

1912-

$1930-$

1898-1903; $1912-$

1902-13: 1914-25*; 1942 -

$1942-$

$1940-41 ; 1948-$

1914-

$1916-42$.

$1933-42$.

$1942-$

1912-

1914-

1911-16;1921-

$1953-$

$1954-$

$1884-89 * \mathrm{k} ; 1890-1943 * \mathrm{c}$; $1898-1903$; $1919-40$; 1963-

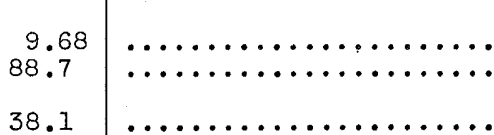

igs $9 \ldots \ldots \ldots \ldots$

$1950-54 ; 1961-$ 1954 ; 1959 -

$1955-$ 


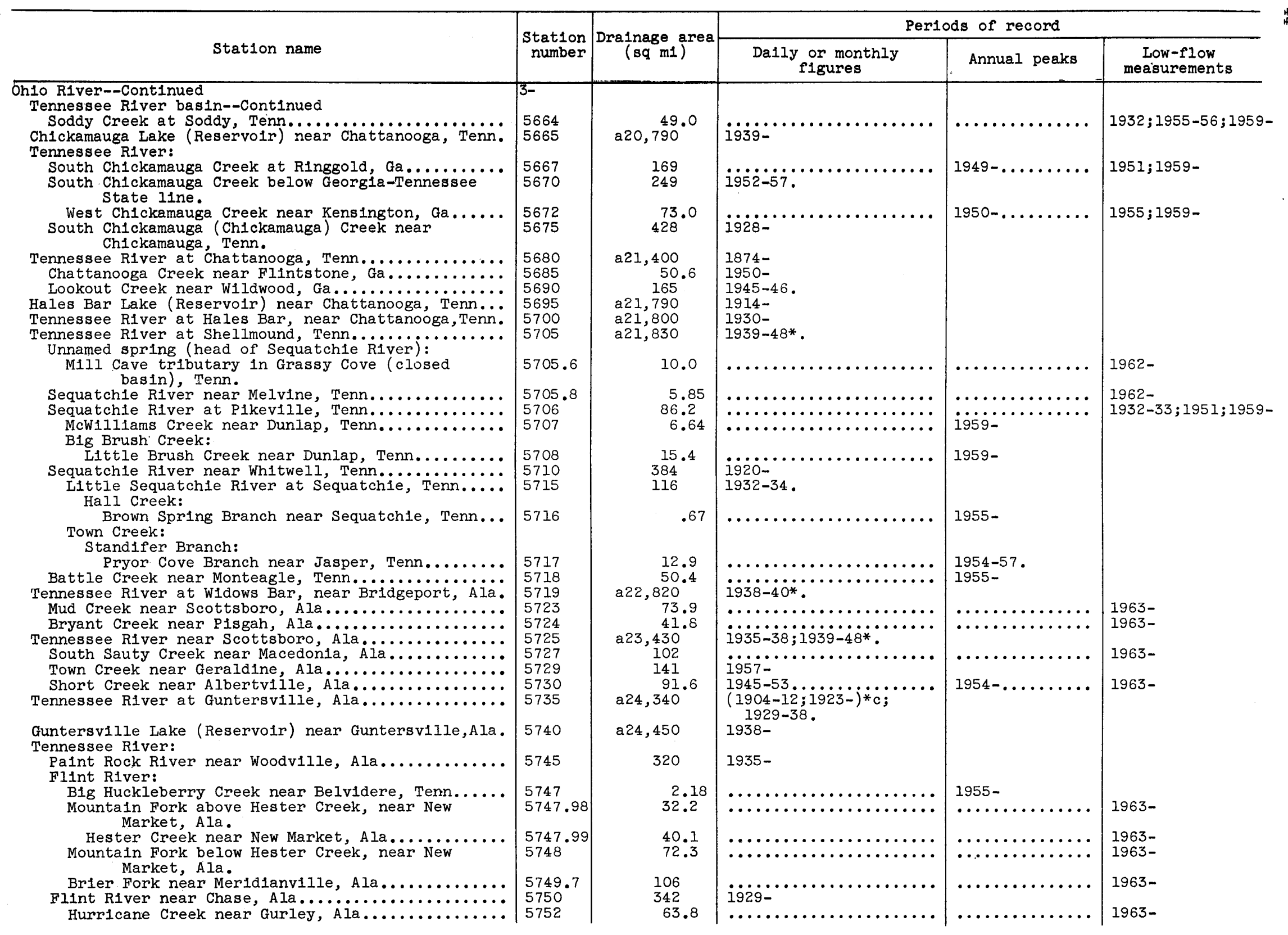


Tennessee River at Whitesburg (Decatur), Ala.......

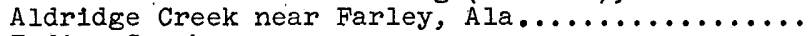
Indian Creek:

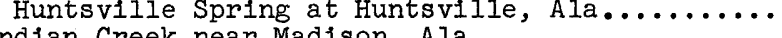

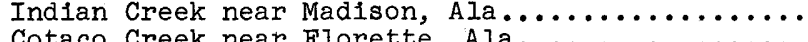

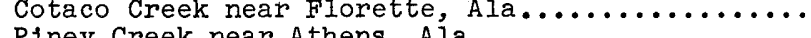
Piney Creek near Athens, Ala....... West Flint Creek:

Elam Creek:

Blowing Spring Branch near Wren, Ala.......

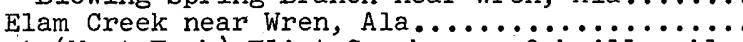
West (West Fork) Flint Creek near Oakvilie, Aia.

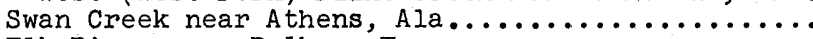

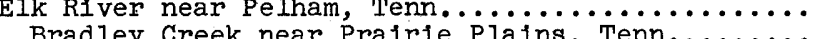
Woods Reservolr at Elk River Dam, near Estiii Springs, Tenn.

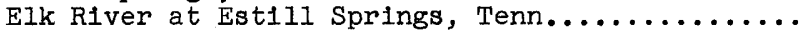

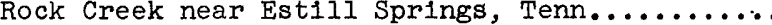
Boiling Fork Creek:

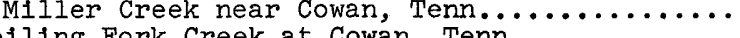

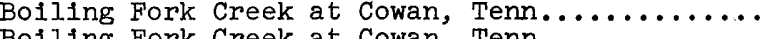

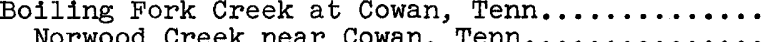
Bolling Fork Creek above Winchester, Tenn....... Bolling Fork Creek at Winchester, Tenn...........

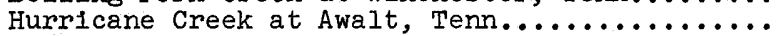
Beans Creek near Lexie Crossroads, Tenn.......... Mulberry Creek:

East Fork Mulberry Creek at Lynchburg, Tenn... West Fork Mulberry Creek at Mulberry, Tenn...

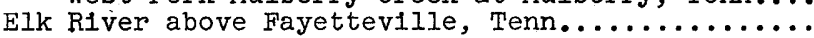
Norris Creek:

Norris Creek tributary near Belleville, Tenn.

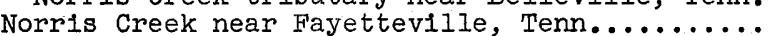

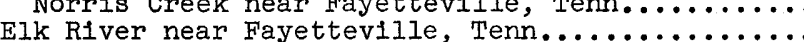

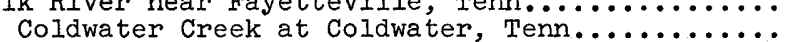

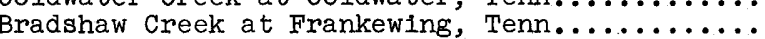

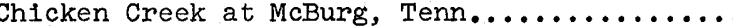

Richland Creek near Cornersvilie, Tenn.........

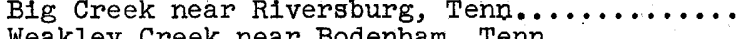

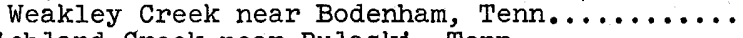

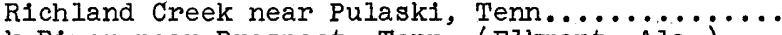
Elk River near Prospect, Tenn. (Elkmont, Ala.)... Shoa 1 Creek:

West Fork Shoal Creek near Minor H1ll, Tenn.. Sugar Creek:

East Fork Sugar Creek near Minor Hill, Tenn...

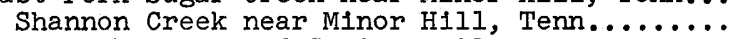

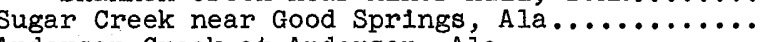
Anderson Creek at Anderson, Ala $\ldots \ldots \ldots \ldots \ldots \ldots \ldots$ Elk River near Rogersville, Ala $\ldots \ldots \ldots \ldots \ldots \ldots \ldots$

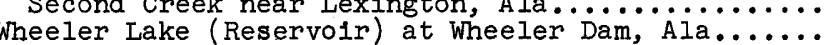

\begin{tabular}{|l}
5755 \\
5757 \\
5760 \\
5761 \\
5761.5 \\
5764 \\
5765 \\
\\
5768 \\
5768.1 \\
5770 \\
5772 \\
5780 \\
5785 \\
5790 \\
\\
5795 \\
5797 \\
5798 \\
5799 \\
5800 \\
5802 \\
5803 \\
5805 \\
5807 \\
5808 \\
5810 \\
5815 \\
5820 \\
5822 \\
5823 \\
5825 \\
5827 \\
5830 \\
5832 \\
5833 \\
5834 \\
5835 \\
5840 \\
5845 \\
5852 \\
5852.5 \\
5852.6 \\
5853 \\
5854 \\
5855 \\
5858 \\
5860 \\
\\
\end{tabular}

\begin{tabular}{|c|c|}
\hline a25, 610 & $\begin{array}{l}1875-* c ; 1924- \\
1960-\end{array}$ \\
\hline $\begin{array}{r}- \\
49.0 \\
158 \\
55.8 \\
86.3\end{array}$ & $\begin{array}{l}1928-32 \\
1959- \\
19359 \ldots \ldots \ldots \ldots \ldots \\
1952-\end{array}$ \\
\hline $\begin{array}{c}1.27 \\
6.69 \\
87.6 \\
28.8 \\
65.6 \\
41.3 \\
263\end{array}$ & $\begin{array}{l}1963- \\
1963- \\
1952-57 ; 1963-\ldots \ldots \ldots \ldots \\
1930 \ldots \ldots \ldots \ldots \ldots \\
1951- \\
1951-59 \ldots \ldots \ldots \ldots \ldots \ldots\end{array}$ \\
\hline $\begin{array}{l}282 \\
41.2\end{array}$ & $\begin{array}{l}1920- \\
\ldots \ldots \ldots \ldots \ldots \ldots \ldots\end{array}$ \\
\hline $\begin{array}{l}4.30 \\
17.0 \\
20.2 \\
10.3 \\
55.9 \\
77.1 \\
24.6 \\
89.7\end{array}$ & 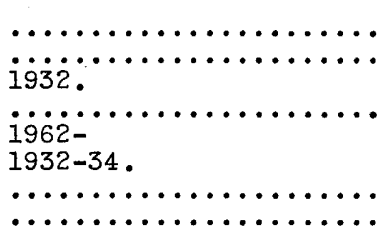 \\
\hline $\begin{array}{r}23.1 \\
41.2 \\
827\end{array}$ & 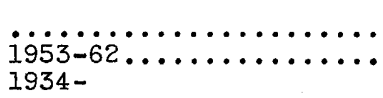 \\
\hline $\begin{array}{l}.034 \\
897\end{array}$ & 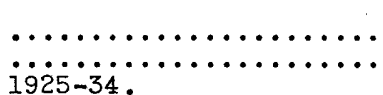 \\
\hline \begin{tabular}{c|}
29.8 \\
36.5 \\
7.66 \\
47.5 \\
85.2 \\
24.4 \\
366 \\
1,784
\end{tabular} & 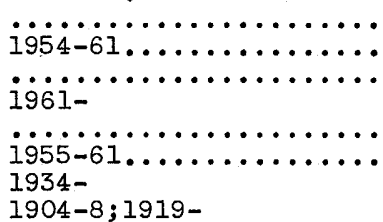 \\
\hline 13.5 & $\ldots \ldots \ldots \ldots \ldots$ \\
\hline $\begin{array}{r}36.6 \\
17.4 \\
152 \\
23.3 \\
2,239 \\
28.1 \\
229,590\end{array}$ & 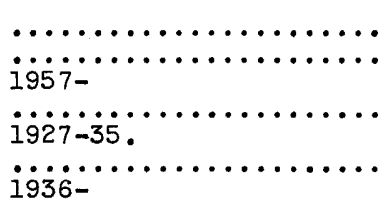 \\
\hline
\end{tabular}

$1963-$

$1958-62$.

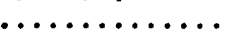

$1960-$

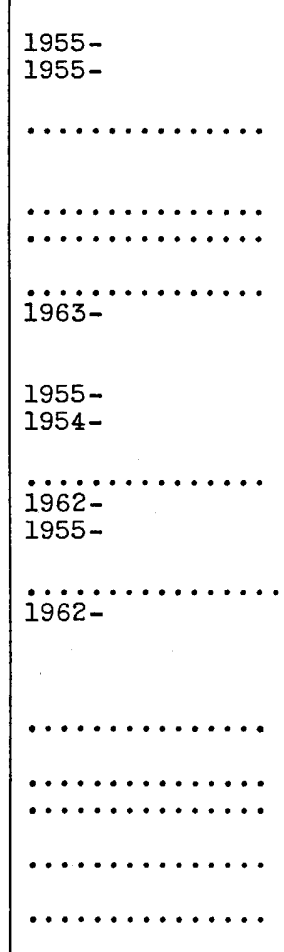

$1962-$

$1959-$

$1959-$

$1963-$

$1963-$ $1953-54 ; 1959-$ $1932-33 ; 1950 ; 1959-$

$1952-54 ; 1959-$

$1952-54 ; 1959-$

$1952-54 ; 1961-$ $1952-54 ; 1961-$

* Gage helghts, or gage helghts and discharge measurements only.

c In reports of U.S. Weather Bureau. 


\begin{tabular}{|c|c|c|c|c|c|}
\hline \multirow[b]{2}{*}{ Station name } & \multirow{2}{*}{$\begin{array}{r}\text { Station } \\
\text { number }\end{array}$} & \multirow{2}{*}{$\begin{array}{c}\text { Drainage area } \\
(\mathrm{sq} \mathrm{m} \mathrm{l})\end{array}$} & \multicolumn{3}{|c|}{ Perlods of record } \\
\hline & & & $\begin{array}{r}\text { Dally or } \\
\text { flg }\end{array}$ & Annual peaks & $\begin{array}{r}\text { Lo } \\
\text { meas }\end{array}$ \\
\hline 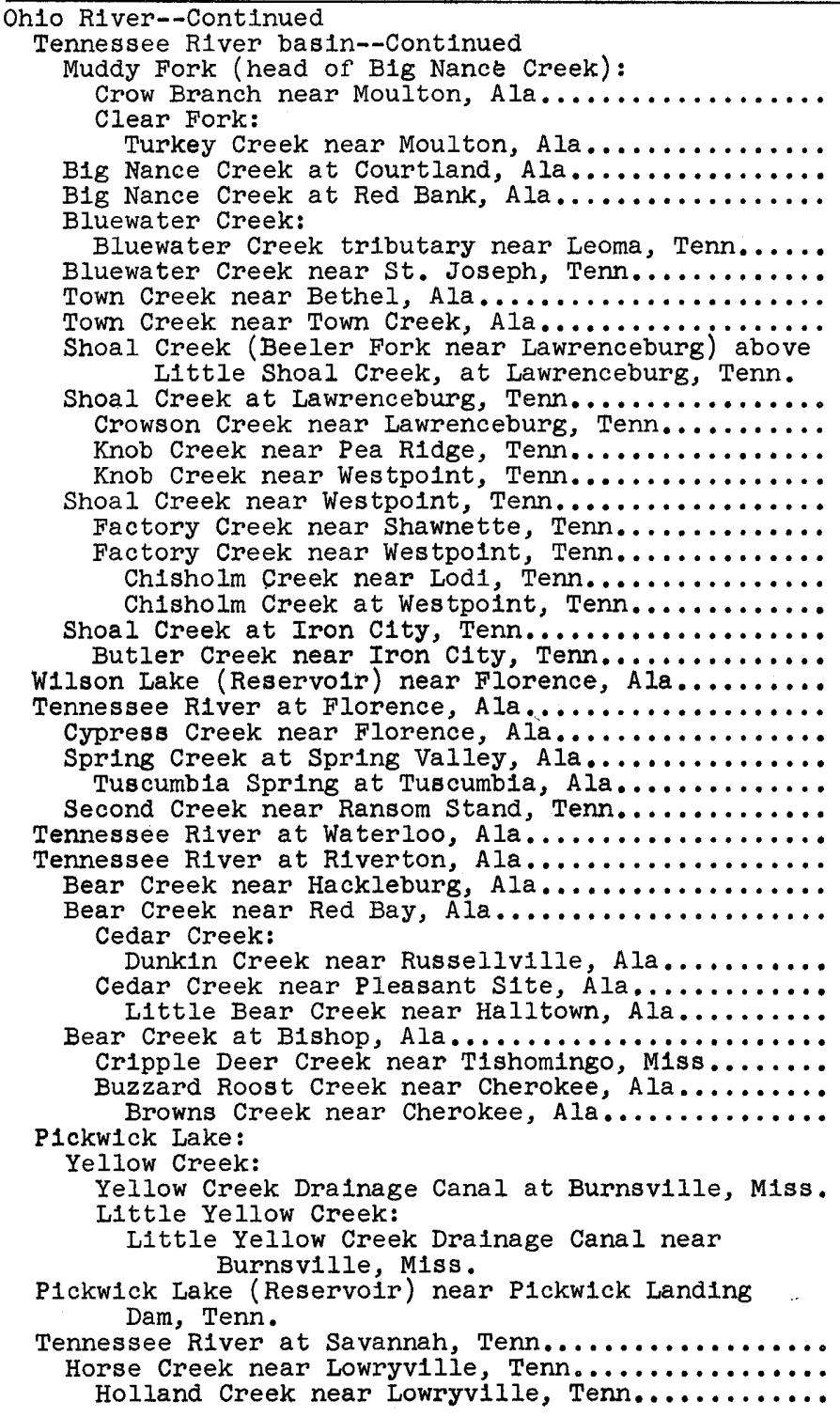 & $\begin{array}{l}5862 \\
5863.5 \\
5865 \\
5870 \\
5872 \\
5873 \\
5873.8 \\
5873.9 \\
5875 \\
5880 \\
5882 \\
5882.4 \\
5882.6 \\
5883 \\
5883.4 \\
5883.6 \\
5883.8 \\
5884 \\
5885 \\
5886 \\
5890 \\
5895 \\
5900 \\
5903 \\
5905 \\
5909 \\
5910 \\
5915 \\
5918 \\
5920 \\
5921.5 \\
5922 \\
5923 \\
5925 \\
5925.5 \\
5926 \\
5926.05\end{array}$ & $\begin{array}{c}a 7 \\
166 \\
188 \\
.49 \\
38.8 \\
126 \\
201 \\
27.0 \\
55.5 \\
18.6 \\
36.7 \\
53.6 \\
176 \\
31.5 \\
71.9 \\
26.8 \\
43.0 \\
348 \\
46.6 \\
230,750 \\
\text { a30, } 810 \\
209 \\
51.8 \\
- \\
42.5 \\
\text { a31,550 } \\
\text { a31,560 } \\
143 \\
263 \\
7.28 \\
189 \\
78.2 \\
667 \\
-38.8 \\
8.71 \\
\text { a32, } 820 \\
\text { a33, } 140 \\
66.7 \\
14.9 \\
46.3 \\
15.4 \\
\end{array}$ & 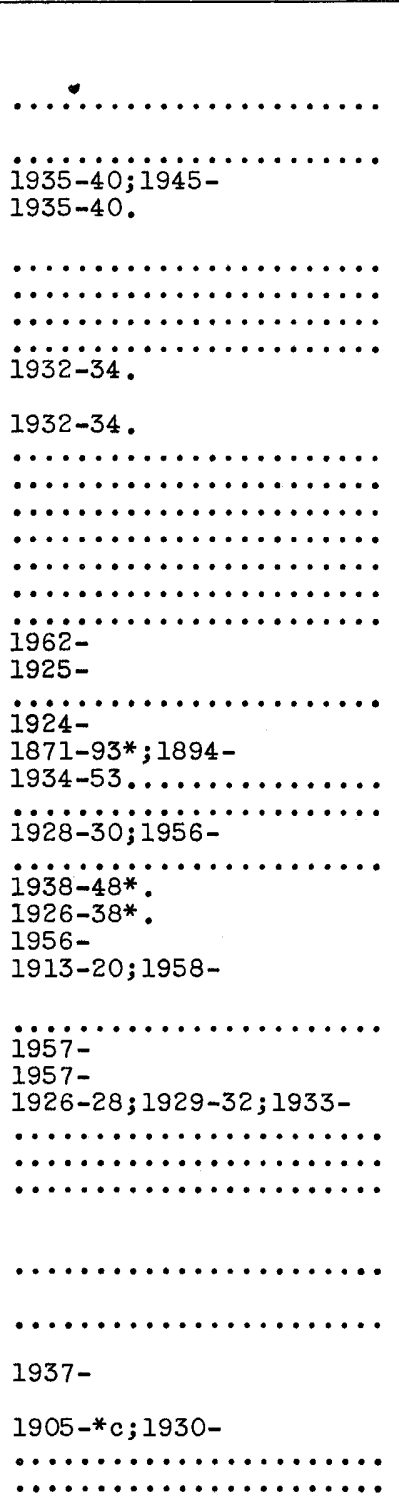 & 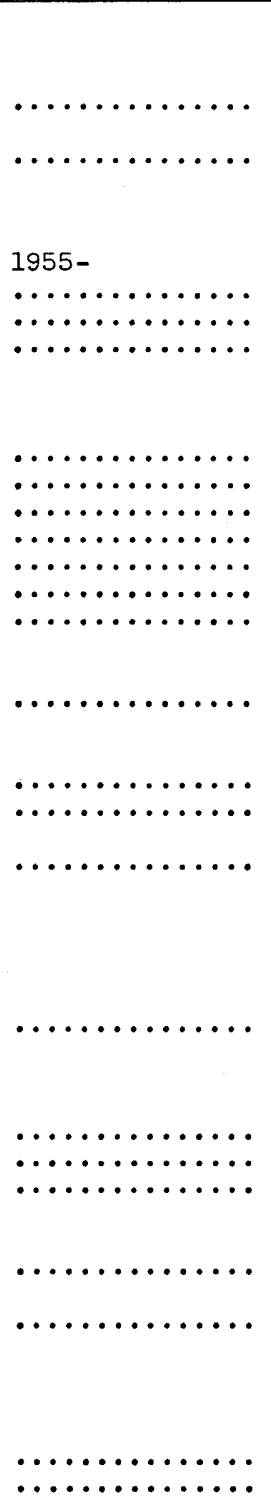 & $\begin{array}{l}1932 ; 1951 ; 1959- \\
1962- \\
1953-56 ; 1962- \\
1962- \\
1962- \\
1953-56 ; 1962- \\
1962- \\
1961- \\
1963- \\
1963- \\
1962-\end{array}$ \\
\hline
\end{tabular}


Horse Creek near Savannah, Tenn.

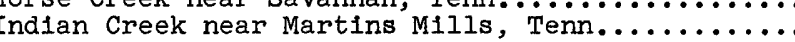

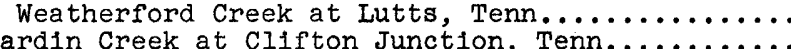

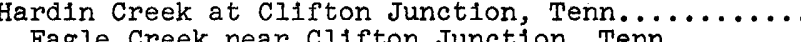

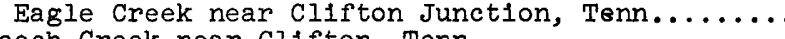

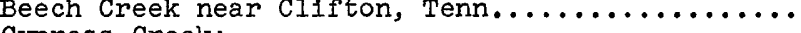

Cypress Creek:

Cypress Creek tributary near Pope, Tenn.........

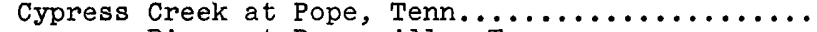

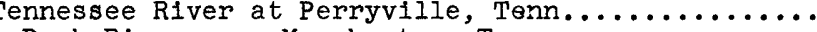

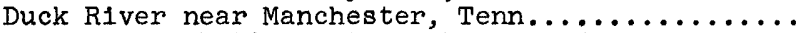

Little Duck River at Manchester, Tenn..........

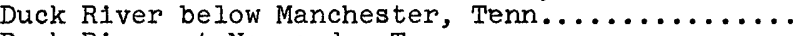

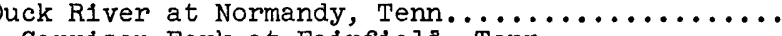

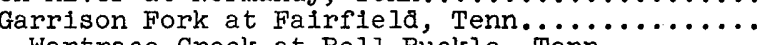

Duck River near Shelbyville, Tenn, $\ldots \ldots \ldots \ldots \ldots$

North Fork Creek:

Weakly Creek near Rover, Tenn............. 5982

Big Rock Creek:

Turner Branch:
Lewisburg Lake near Lewisburg, Tenn........

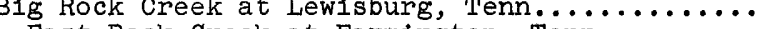

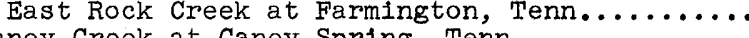

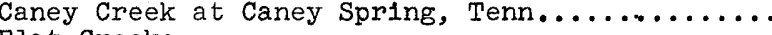

Flat Creek:

Little Flat Creek tributary near Rally

Duck River

Rutherford Creek near Carters Creek, Tenn........ Big Bigby Creek near Sandy Hook, Tenn........... Big Bigby Creek near Mount Pleasant, Tenn..........

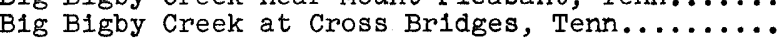

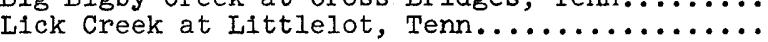

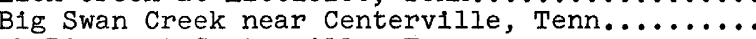

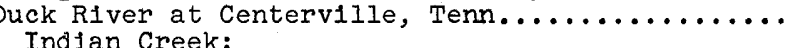

Moss Spring Hollow near Centerville, Tenn.... 6021 Piney River at Vernon, Tenn............... 6025

Tumbling Creek near Hurricane Mili, Tenn........ 602

Duck River above (near) Hurricane Milis, Tenn...... 6030

Hurricane Creek at Hurricane Mills, Tenn....... 6035

Buffalo River at Henryville, Tenn............ 6035.5 Saw Creek at Barnesville, Tenn.......................6 6035.6

Buffalo River above Riverside, Tenn............ 6036

Little Buffalo River near Napier, Tenn....... 6036.6

Chief Creek at Napler, Tenn......................... 6036.9

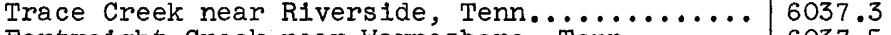

Fortyeight Creek near Waynesboro, Tenn........ 6037.5

Fortyeight Creek at Topsy, Tenn $\ldots \ldots \ldots \ldots$ reen River:

Cha lk Creek near Waynesboro, Tenn.......... 6038

Green River at Waynesboro, Tenn................. 6038.5

* Gage helghts, or gage helghts and discharge measurements only. a Approximately.

In reports of U.S. Weather Bureau.

$f$ In files of Geological Survey.

\section{$114 \quad$ 1929-34.}

84.4
25.8 $\ldots \ldots \ldots \ldots \ldots \ldots \ldots \ldots$

24.7

16.75

16.8

55.2

36.4

107

66.3

16.3

481

9.46

al.2

24.9
43.1

28.9

63

1,208

68.8

17.5

25.8

99.8

154
, 048

3.68

193.

51
2,557

75.

12.1

126

37.7

24.6

19.1

56.6

4.88
22.8

$\ldots \ldots \ldots \ldots \ldots \ldots$

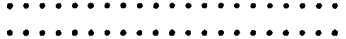

$\ldots \ldots \ldots \ldots \ldots \ldots$

$\ldots \ldots \ldots \ldots$

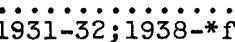

$1932-34$.

$1932-34$

$1920-31$

$1920-31$.

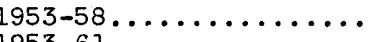
............... $1933-$

-

1933-38*

$1953-61 . \ldots \ldots \ldots \ldots$.

$\ldots \ldots \ldots \ldots \ldots$

$(1887-95 ; 1911-) * c$;

$1904-8 ; 1920-$

$1953-58$

1953-57.

$1938-39$.

$\ldots \ldots \ldots \ldots \ldots$ $1 \ddot{9} \ddot{9}-5 \dot{5}$.

\section{i்ㄹㅎ-}

ïㄹㄹㅇ-

$1932-33$.

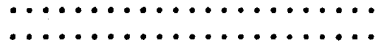

$\ldots \ldots \ldots \ldots \ldots \ldots \ldots$

$\cdots \ldots \ldots \ldots \ldots \ldots \ldots$

$\cdots \cdots \cdots \cdots$

.....................

$\ldots \ldots \ldots \ldots \ldots \ldots \ldots$

$\ldots \ldots \ldots \ldots \ldots \ldots \ldots \ldots \ldots \ldots \ldots \ldots$
1962

1962

$1928 ; 1962-$

$i 955 \ldots \ldots \ldots \ldots \ldots$ 1928;

............ 1962

$1955-$

$1955-\ldots \ldots . . .$.

$1953-$

1959-

$1962-$

$1955-$

1962

$1954-$

.................

$1955-$

$1959-$

$\ldots \ldots \ldots \ldots$

$1955-$

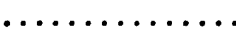

1953

......... 1952-53;1961-

a......... $1963-$

a........

.......... 1953-54;1964-

$\ldots \ldots \ldots \ldots$ 1961-

$1955-57 ; 1960-$ 


\begin{tabular}{|c|c|c|c|c|c|}
\hline \multirow[b]{2}{*}{ Station name } & \multirow{2}{*}{$\begin{array}{l}\text { Station } \\
\text { number }\end{array}$} & \multirow{2}{*}{$\begin{array}{c}\text { Drainage area } \\
(\mathrm{sq} \mathrm{m} 1)\end{array}$} & \multicolumn{3}{|c|}{ Periods of record } \\
\hline & & & $\begin{array}{c}\text { Dally or monthly } \\
\text { flgures }\end{array}$ & Annual peaks & $\begin{array}{l}\text { Low-flow } \\
\text { measurements }\end{array}$ \\
\hline 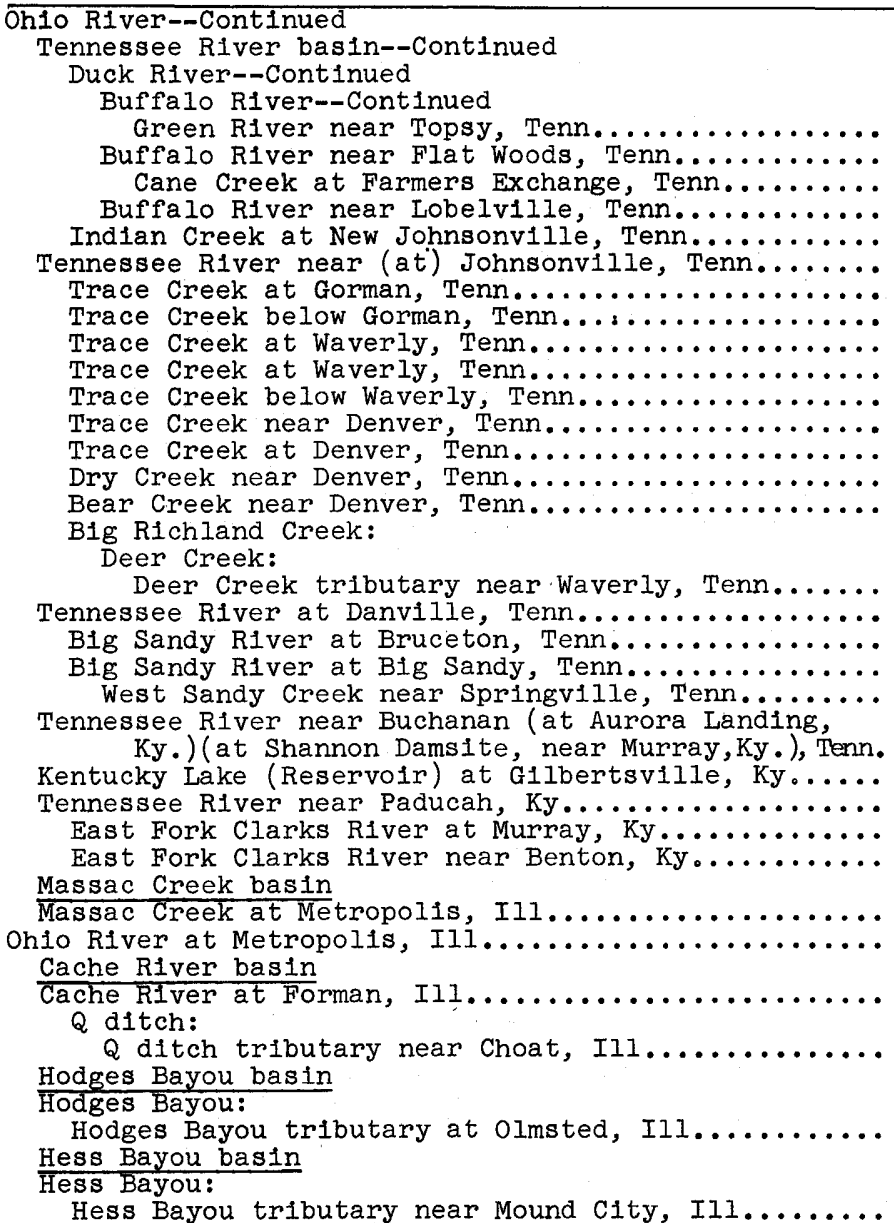 & $\begin{array}{l}6039 \\
6040 \\
6042 \\
6045 \\
6049 \\
6050 \\
6052 \\
6053 \\
6054 \\
6055 \\
6055 \cdot 2 \\
6055.5 \\
6055 \cdot 6 \\
6055.8 \\
6056 \\
\\
6057 \\
6060 \\
6065 \\
6070 \\
6072 \\
6075 \\
6090 \\
6095 \\
6100 \\
6105 \\
6112 \\
6115 \\
6120 \\
6122 \\
6135\end{array}$ & $\begin{array}{c}1.04 \\
a 38,970 \\
205 \\
379 \\
47.9 \\
a 39,730 \\
a 40,200 \\
a 40,200 \\
89.7 \\
227 \\
37.4 \\
a 203,000 \\
243 \\
.278 \\
4.67\end{array}$ & 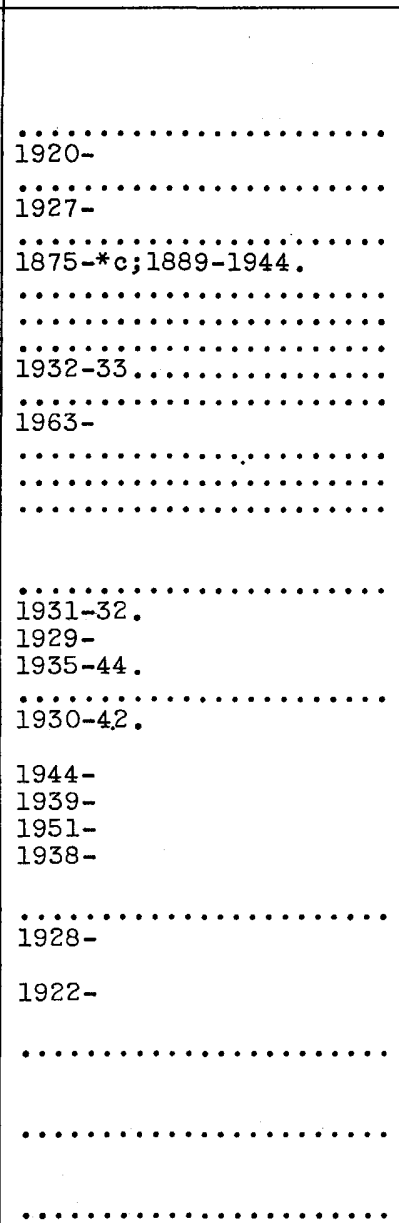 & 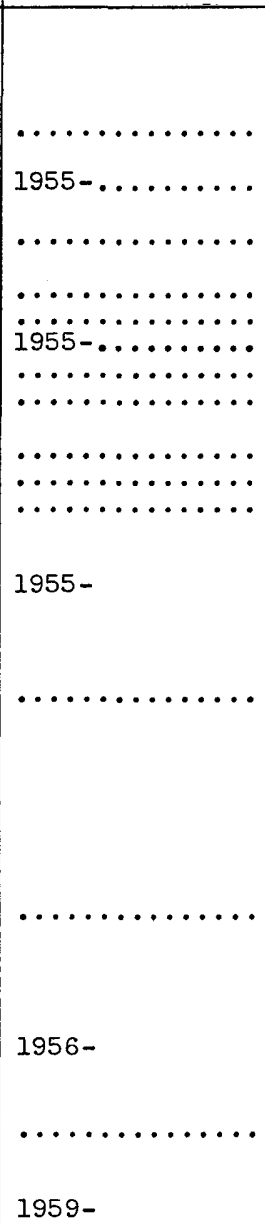 & $\begin{array}{l}1944 ; 1964- \\
1956 ; 1959- \\
1964- \\
\\
1964- \\
1964- \\
1953-56 . \\
1964- \\
1964- \\
1964- \\
1964- \\
1964-\end{array}$ \\
\hline
\end{tabular}

* Gage helghts, or gage heights and discharge measurements only.

a Approximately.

$c$ In reports of U.S. Weather Bureau. 
Abers Creek, $\mathrm{Pa} \ldots \ldots \ldots \ldots \ldots \ldots \ldots \ldots \ldots \ldots \ldots$

Akeley Run, $\mathrm{Pa}$

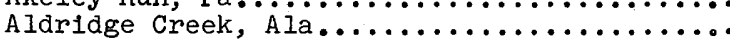

Allegheny River, $N, \mathrm{Y} .-\mathrm{Pa} \ldots \ldots \ldots \ldots \ldots \ldots . \ldots 4,5,6,7$

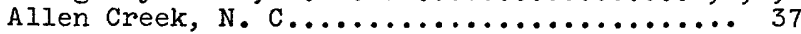

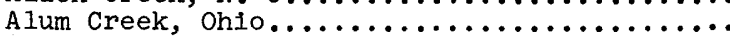

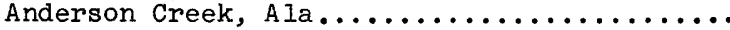

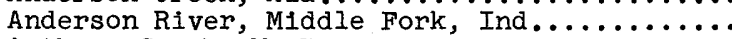

Anthony Creek, $w_{\text {. Va }} \ldots \ldots \ldots \ldots \ldots \ldots \ldots \ldots \ldots$

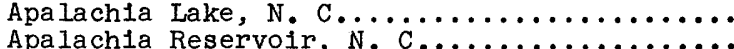

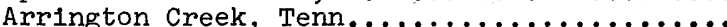

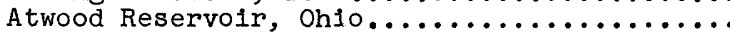

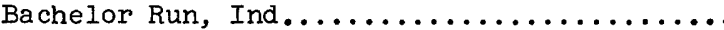

Back Fork, W. Va.

Bacon Creek, Ky.

Ball Creek, N. Y

Banning Lake, Ind

Barnes Run, Ohio.

Barren Fork, Tenn

Bartous Creek, Tenn

Battle Creek, Tenn.

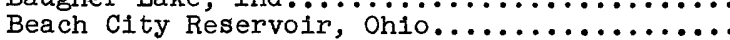

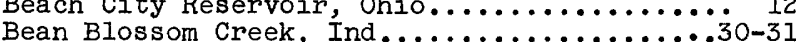

Beans Creek, Tenn.................. 45

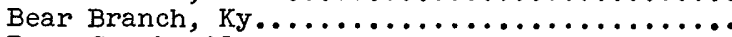

Bear Creek, Ala.

Bear Creek, Ind. (tributary to Bean Blossom Creek).

Bear Creek, Ind. (tributary to Lobloliy Creek).

Bear Creek, Ky

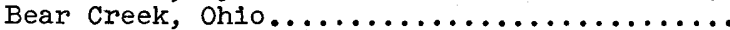

Bear Creek, Tenn. (tributary to East Fork

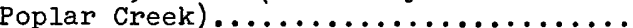

Bear Creek, Tenn. (tributary to Tennessee River).

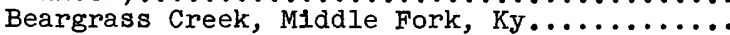

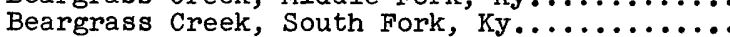

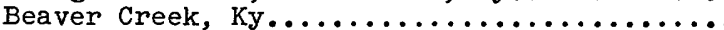

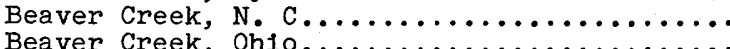

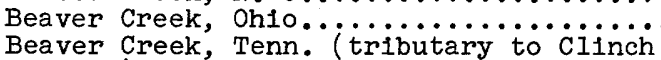

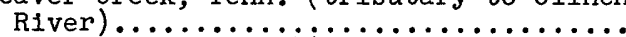

Beaver Creek, Tenn. tributary to soüh

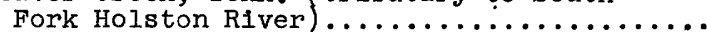

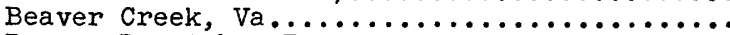

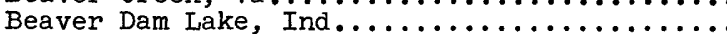

Beaver River, $\mathrm{Pa} . . . \ldots \ldots \ldots \ldots \ldots \ldots \ldots \ldots$

Beaverdam Creek, Va. (tributary to Laurei

Creek)

Beaverdam Creek, $\mathrm{Va}$ (tributary to Littie

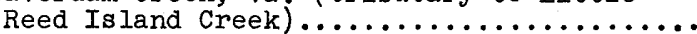

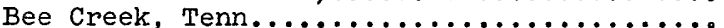

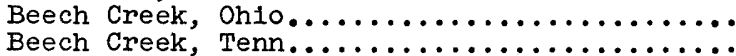

Beech Fork, Ky .....

Beetree Creek, N.

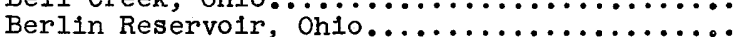

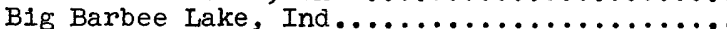

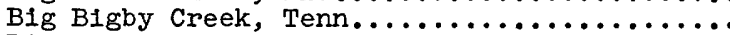

B1g Blue River, Ind.

B1g Chapman Lake, Ind.

Big Coal River, W. Va.

B1g Creek, IIl.

B1g Creek

Big Creek, Ind. (tributary to Graham Creek).

Big Creek, Tenn. (tributary to Clinch RIver).

Big Creek, Tenn. (tributary to Holston River)

Big Creek, Tenn. (tributary to Richland

Creek).

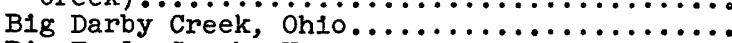

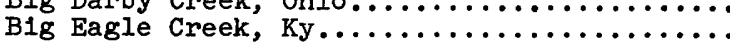

Big Eagle Creek, Tenn................ 34

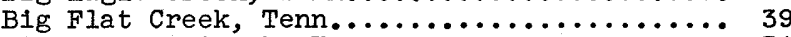

Big Hickory Creek, Tenn............... 34

Big Huckleberry Creek, Tenn.............. 44

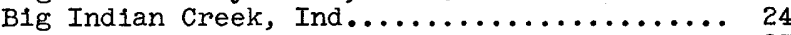

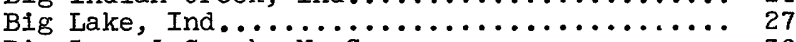

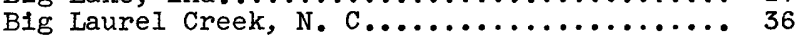

B1g Limestone Creek, Tenn.............. 37

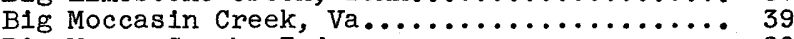

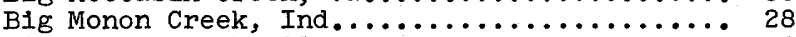

Blg Nance Creek, Ala................... 46

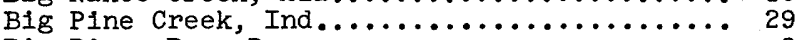

Big Piney Run, Pa................... 9

B1g Reed Island Creek, Va................ 15

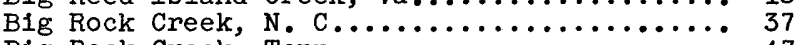

Big Rock Creek, Tenn................ 47

Big Run, $\mathrm{Pa}$. (tributary to Iittie Sandy

Creek ......................... 6

B1g Run, $\mathrm{Pa}$ (tributary to Shenango River)... 10

Big Sandy Creek, $\mathrm{w}$. Va. (tributary to cheat River)

Blg Sandy Creek, $\hat{w}$. Va. (tributary to Ëik

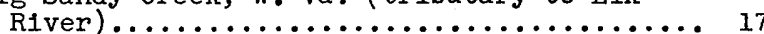

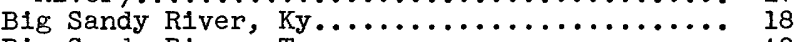

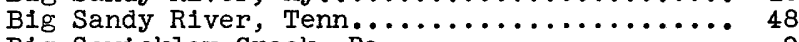

Big Sewickley Creek, Pa................... 9

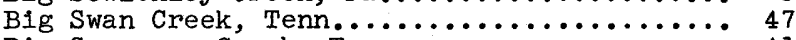

Blg Sycamore Creek, Tenn..................... 41

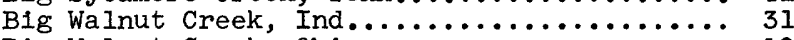

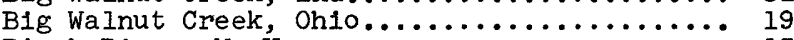

Birch R1ver, W. Va..................... 17

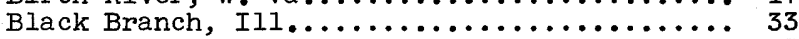

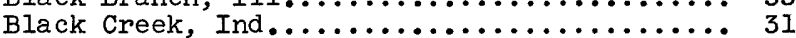

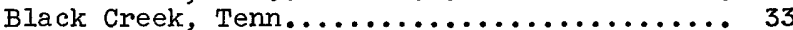

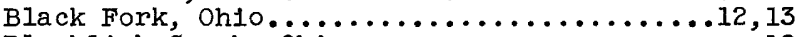

Blacklick Creek, Ohio.................. 19

Blacklıck Creek, $\mathrm{Pa} \ldots \ldots \ldots \ldots \ldots \ldots \ldots \ldots \ldots \ldots \ldots \ldots \ldots{ }_{8}$

Blackwater River, North Fork, w. va......... 8

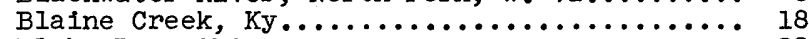

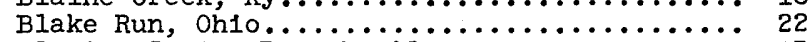

Blowing Spring Branch, Ala............. 45

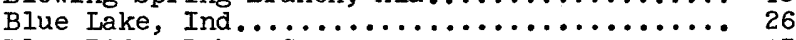

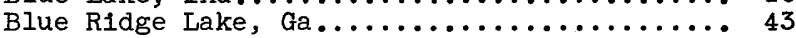

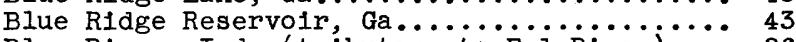

Blue River, Ind. (tributary to Eei River) ... 26

Blue River, Ind. tributary to Ohio River $\ldots . .25$

Blue River, Ind. (tributary to White River).. 31

Blue River, Middle Fork, Ind............. 24

Bluegrass Creek, Ill.................. 29

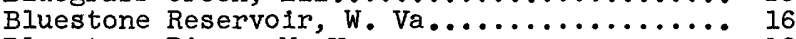

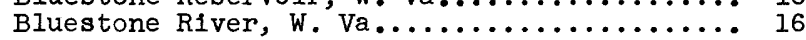

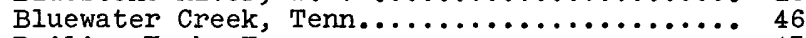

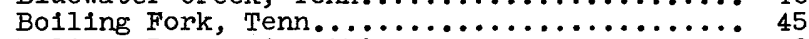

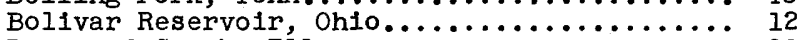

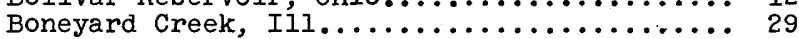

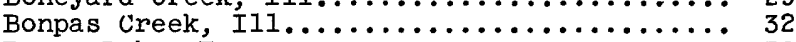

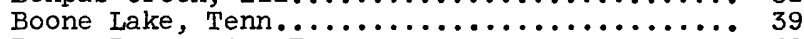

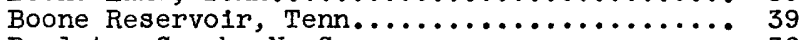

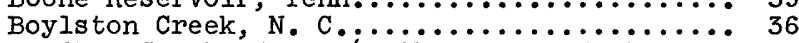

Bradley Creek, Tenn, (tributary to East

Fork Stones River) ............... 35

Bradley Creek, Tenn. (tributary to Eik River) 45

Bradshaw Creek, Tenn.................. 45

Brandyw1ne Creek, Ind................................ 31

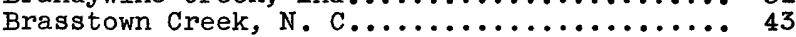

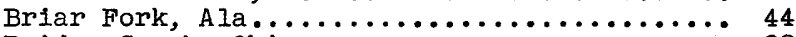

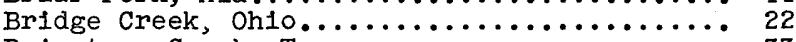

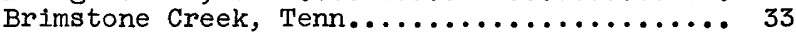

Brokenstraw Creek, $\mathrm{Pa} \ldots \ldots \ldots \ldots \ldots \ldots \ldots \ldots \ldots \ldots \ldots{ }_{4}$

Brown Spring Branch, Tenn................. 44

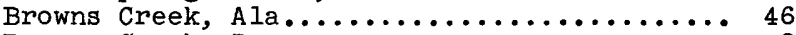

Browns Creek, Pa...................... 8

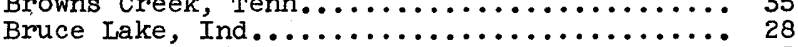

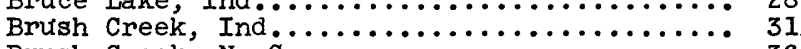

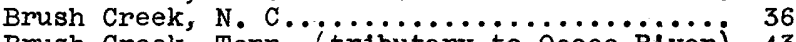

Brush Creek, Tenn. (tributary to Ocoee River) 43 Brush Creek, Tenn. tributary to Watauga 
Page

Brush Run, $\mathrm{Pa}, \ldots \ldots \ldots \ldots \ldots \ldots \ldots \ldots \ldots \ldots$, I1

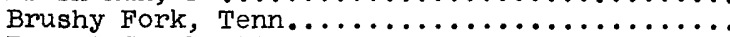

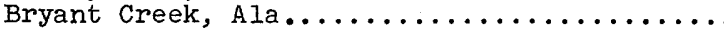

Brymer Creek, Tenn.

Buck Creek, Ind. (tributary to sugar creek).

Buck Creek, Ind. (tributary to White River)..

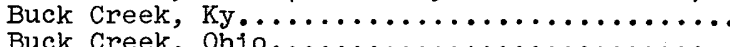

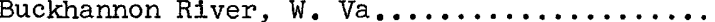

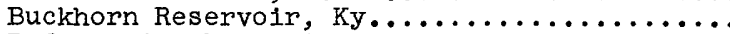

Buffalo Creek, N. C........................

Buffalo Creek, ohio......................

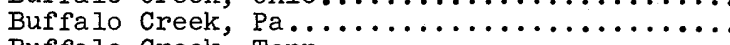

Buffalo Creek, Tenn.

Buffalo Creek, w. Va. (tributary to

Monongahela River).

Buffalo Creek, W. Va. (tributary to ohio

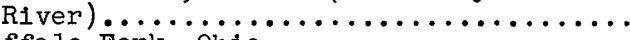

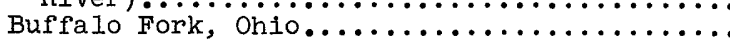

Buffalo River, Tenn..................47,48

Bullrun Creek, Tenn...................... 41

Burr Oak Reservoir, Ohio................. 14

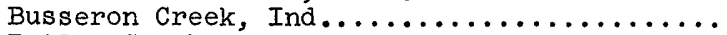

Butler Creek, Tenn...

Buzzard Roost Creek, Ala....................

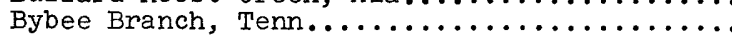

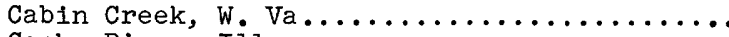

Cache River, Iil...

Caesar Creek, Ohio.

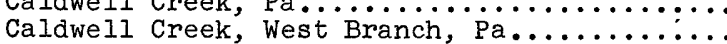

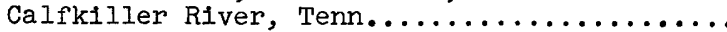

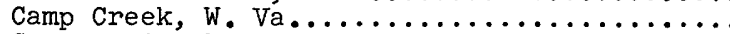

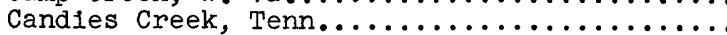

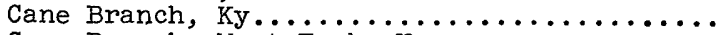

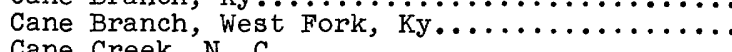

Cane Creek, N. C.............. (tributary to Buffaio

River).

Cane Creek, Tenn. (tributary to Caney Fork).

Cane Creek, Tenn. (tributary to Hiwassee River)...

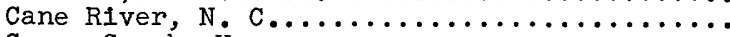

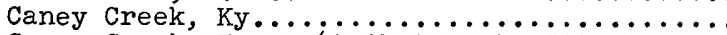

Caney Creek, Tenn. (tributary to ciinch

River)...

Caney Creek, Tenn. (tributary to Duck River).

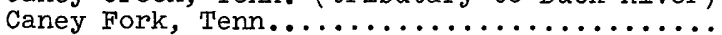

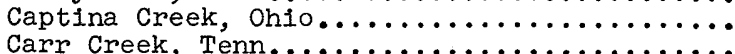

Carr Creek, Tenn.

Carr Fork, Ky...

Carr Lake, Ind $\ldots \ldots \ldots \ldots \ldots \ldots \ldots \ldots \ldots \ldots$

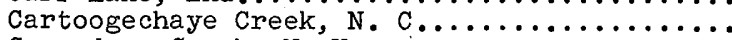

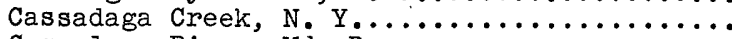

Casselman River, Md $-\mathrm{Pa} \ldots \ldots \ldots \ldots \ldots \ldots \ldots \ldots$

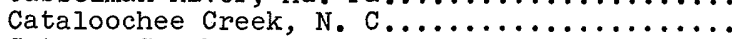

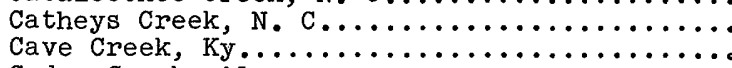

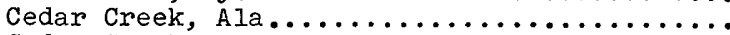

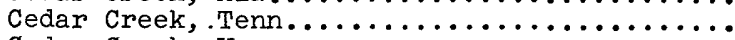

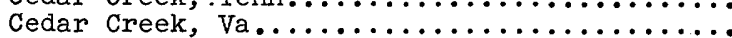

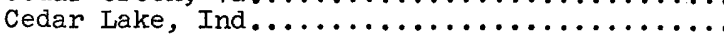

Cedarville spring, Va $\ldots \ldots \ldots \ldots \ldots \ldots \ldots \ldots \ldots$

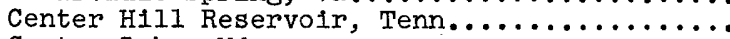

Center Lake, Md. ${ }_{\text {Chadakoin River }} \ldots \ldots \ldots \ldots \ldots \ldots \ldots \ldots \ldots$

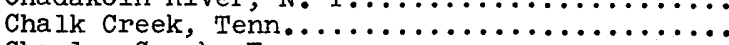

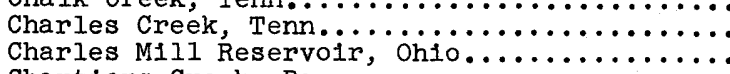

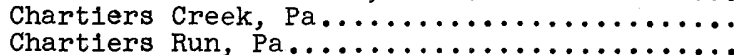

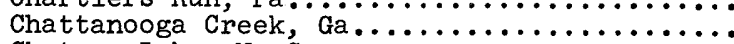

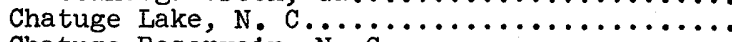

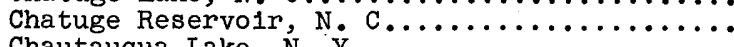

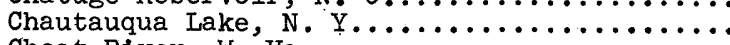

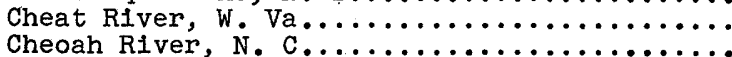

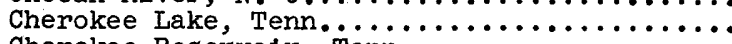

Cherokee Reservolr, Tenn.

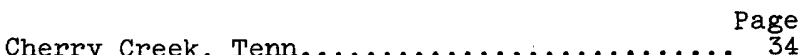

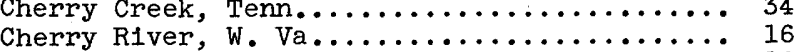

Cherry River, North Fork, W. Va.......... 16

Cherry River, South Fork, w. Va............. 16

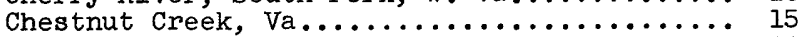

Chickamauga Creek, Tern.............. 44

Chickamauga Lake, Tenn................... 44

Chickamauga Reservoir, Tenn............. 44

Chicken Creek, Tenn................. 45

Chlef Creek, Tenn................. 47

Chilhowee Iake, Tenn................ 40

Chippewa Creek, Ohio.................. 12

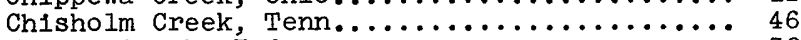

Cicero Creek, Ind.................... 30

Clarion River, $\mathrm{Pa} \ldots \ldots \ldots \ldots \ldots \ldots \ldots \ldots \ldots \ldots{ }_{5}$

Clarion River, East Branch, Pa............... 5

Clarion River, West Branch, Pa........... 5

Clarion River Reservolr, East Branch, $\mathrm{Pa} \ldots . .55$

Clarks River, East Fork, Ky............. 48

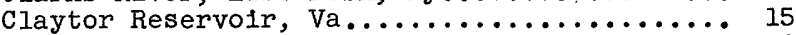

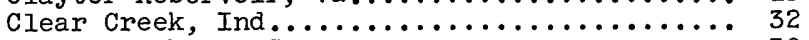

Clear Creek, N. C................... 36

Clear Creek, Ohio (tributary to Great Miami

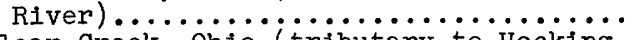

Clear Creek, Ohio (tributary to Hocking

River) .........

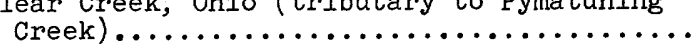

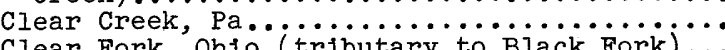

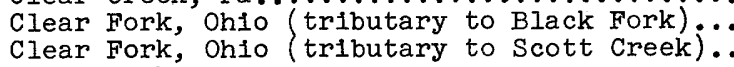

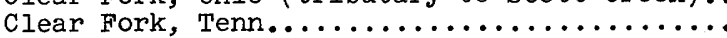

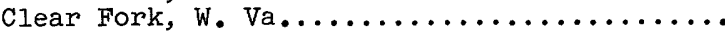

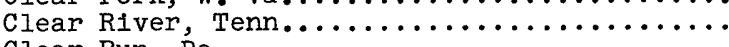

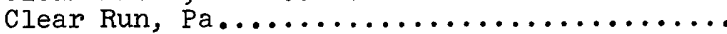

Clear Shade Creek, $\mathrm{Pa} \ldots \ldots \ldots \ldots \ldots \ldots \ldots \ldots \ldots \ldots \ldots,{ }^{6}$

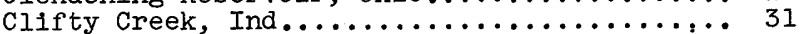

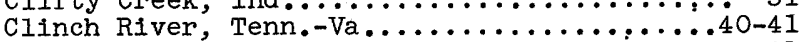

Clinch River, North Fork, Va............ 41

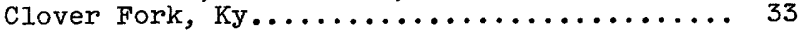

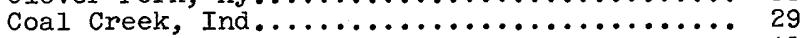

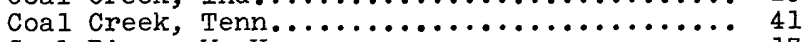

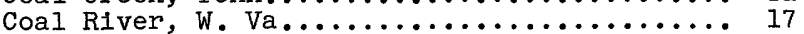

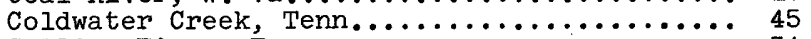

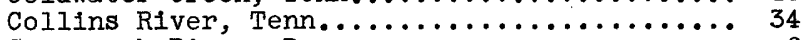

Conemaugh River, $\mathrm{Pa} \ldots \ldots \ldots \ldots \ldots \ldots \ldots \ldots \ldots \ldots{ }^{6}$

Conemaugh River Reservoir, $\mathrm{Pa} \ldots \ldots \ldots \ldots \ldots \ldots{ }_{6}$

Conewango Creek, N. Y.-Pa............. 4

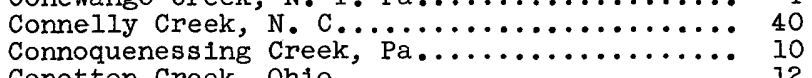

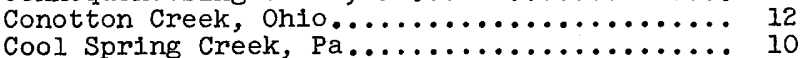

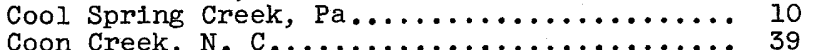

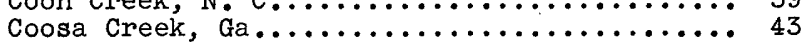

Copper Creek, Va............................... 4

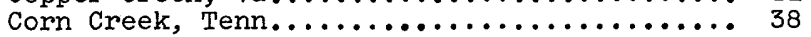

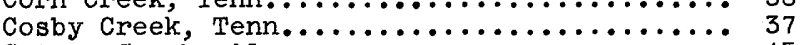

Cotaco Creek, Ala .............................. 45

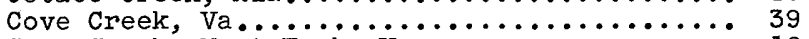

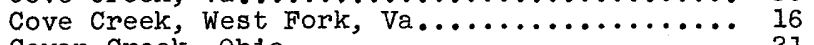

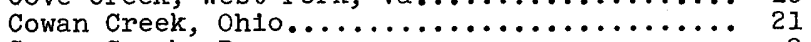

Coxes Creek, Pa........................... 9

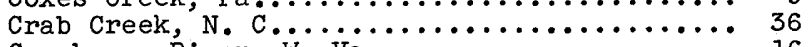

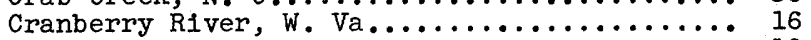

Cranenest River, Va........................... 18

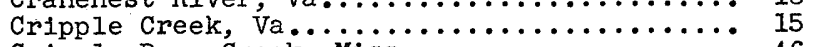

Cripple Deer Creek, Miss............... 46

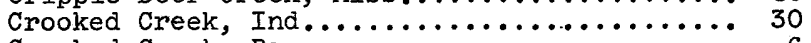

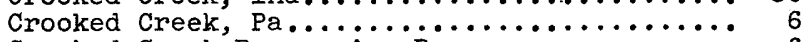

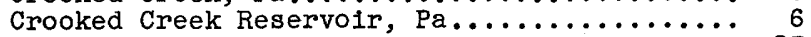

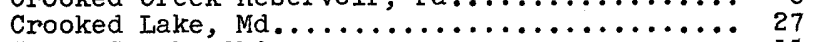

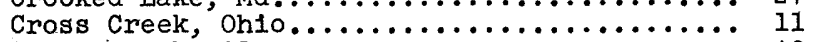

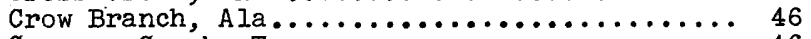

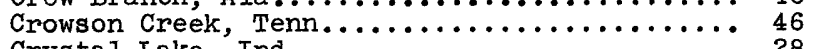

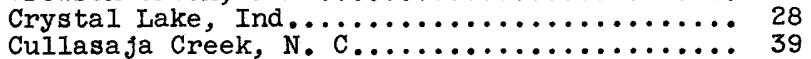

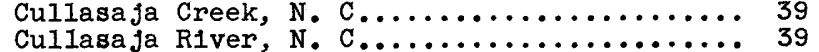

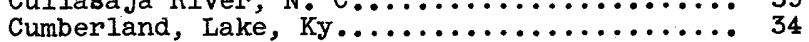

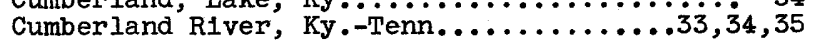




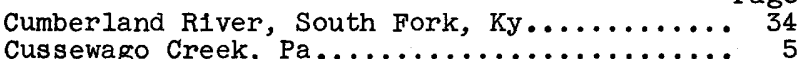

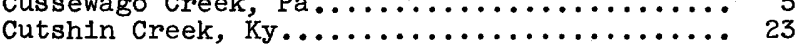

Cypress Creek, Ala.-Tenn............46,47

Daddy Creek, Tenn.

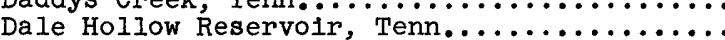

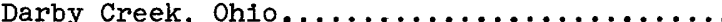

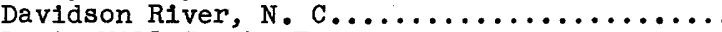

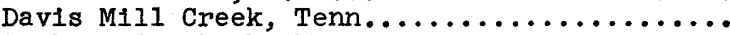

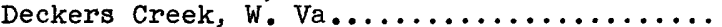

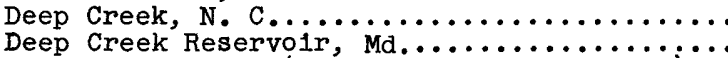

Deer Creek, Ind. (tributary to Mili Creek)...

Deer Creek, Ind. tributary to Wabash River).

Deer Creek, ohio (tributary to Mahoning

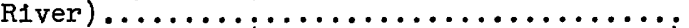

Deer Creek, ohio (tributary to scioto River).

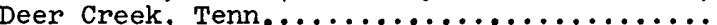

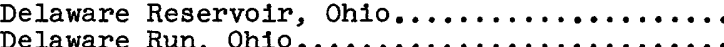

Ohio.

Dicks Creek,

Dicks River, Ky .

Dilion Reservoir, ohio $\ldots \ldots \ldots \ldots \ldots \ldots \ldots \ldots \ldots \ldots$

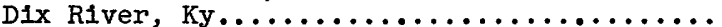

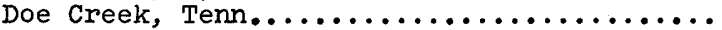

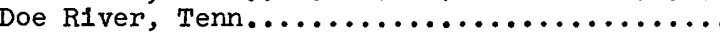

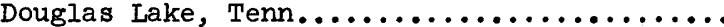

Douglas Reservoir, Tenn.

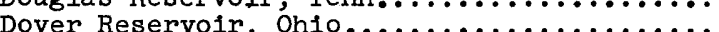

Drake Run, $\mathrm{Pa} . \ldots \ldots \ldots \ldots \ldots \ldots \ldots \ldots \ldots \ldots$

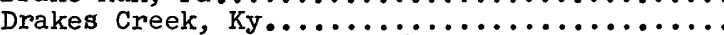

Drakes Creek, Tenn.

Driftwood RIver, Ind . . . . . . . . . . . . . . . . .

Drunkard Creek, $\mathrm{Pa} \ldots \ldots \ldots \ldots \ldots \ldots \ldots \ldots \ldots \ldots \ldots \ldots$

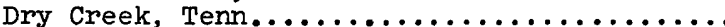

Dry Fork, W. Va. (tributary to Monongahela River)

Dry Fork, $w_{\text {. }}$ Va. (tributary to Tug Fork).....

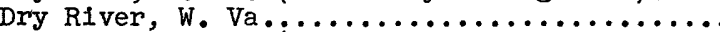

Duck Creek, on1o (tributary to Mahoning

RIver).

Duck Creek, ohio (tributary to ohio Riverj)...

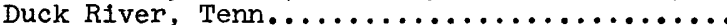

Dums Creek, I11.

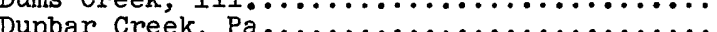

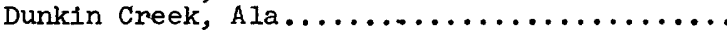

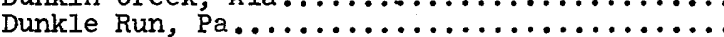

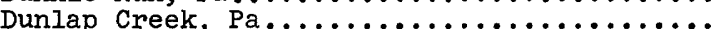

Eagle Creek, Ind.

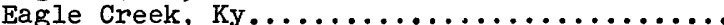

Eagle Creek, Ohio (tributary to Mahoning River)

Eagle creek, onio (tributary to ohio River).

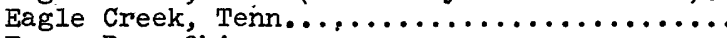

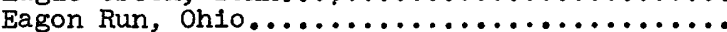

East Branch or Fork. See name of main stream.

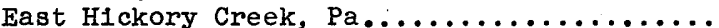

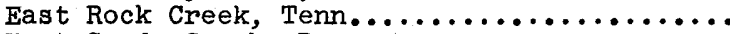

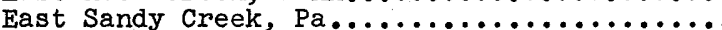

Eel RIver, Ind. (tributary to Big Walnut Creek).

Eel River, Ind. (tributary to Wabash Riverj 26

Eel River, Ind. (tributary to White River)...

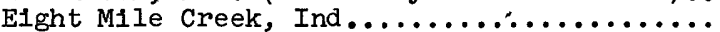

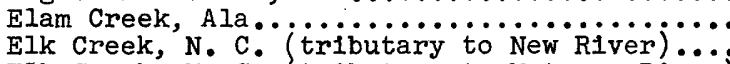

Elk Creek, N. C. (tributary to Watauga River)

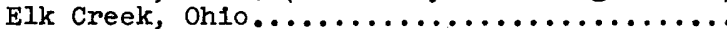

EIk Creek, W. Va.

Elk Fork, Tenn.

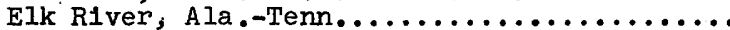

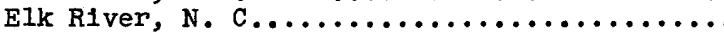

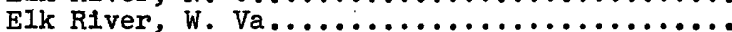

Elkhorn Creek, Ky................... 24

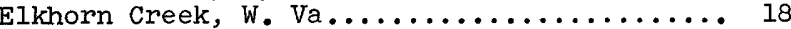

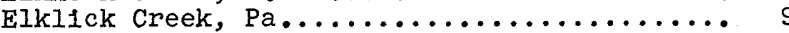

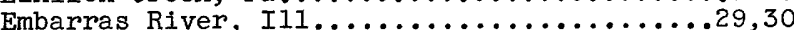

Embarras River, North Fork, III......... 30

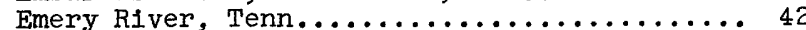

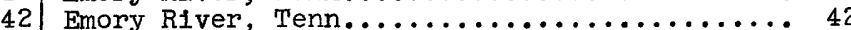

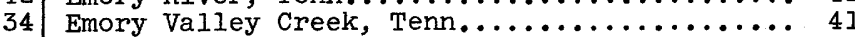

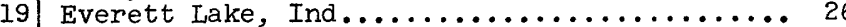

Factory Creek, Tenn.................. 45

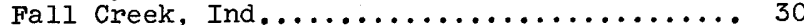

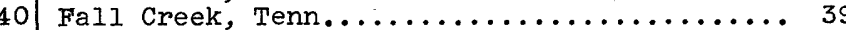

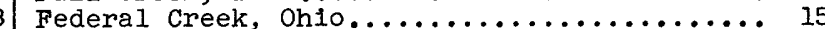

31 Fightingtown Creek, Ga............... 43

First Creek, Tenn.................. 39

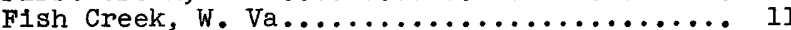

Fish Creek, Pennsylvania Fork, Pa.......... II

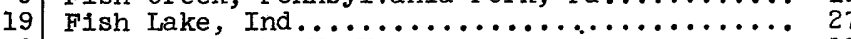

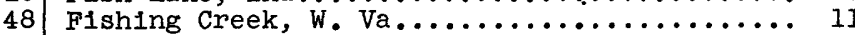

19 Fivemile Creek, N. Y..................

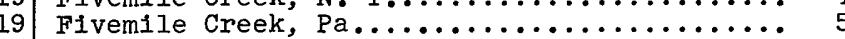

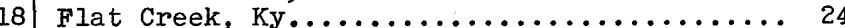

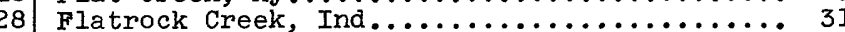

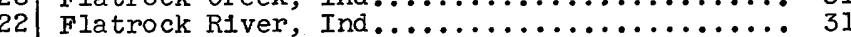

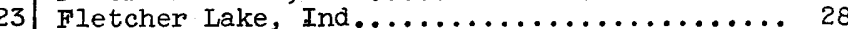

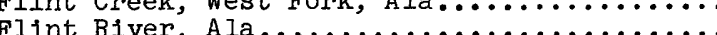

Floyds Fork, Ky

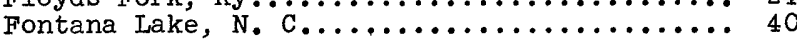

Fontana Reservolr, N. C................ 40

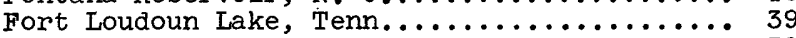

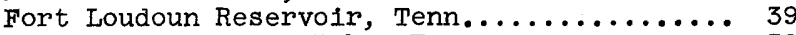

Fort Patrick Henry Lake, Tenn............ 39

Fort Patrick Henry Reservolr, Tenn......... 39

Fortyeight Creek, Tenn............... 47

Four Mile Creek, Oh10............... 22

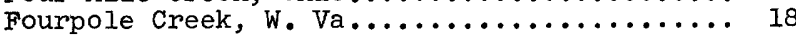

Fourteenmlle Creek, Ind................ 24

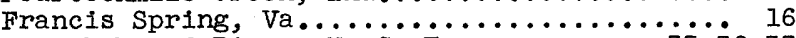

French Broad River, $\mathrm{N}$. C.-Tenn.................35, 37

French Broad River, East Fork, N. C........

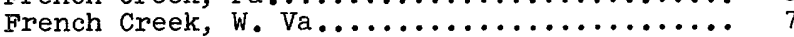

Galbreath Creek, $N$. C............... 40

Gandy Creek, W. Va................... 8

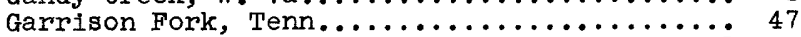

Gauley River, w. Va ...................... 17

Georges Creek, $\mathrm{Pa} \ldots \ldots \ldots \ldots \ldots \ldots \ldots \ldots \ldots{ }_{8}$

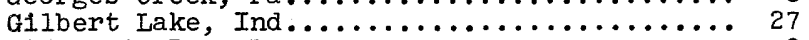

Gillespie Run, Pa................. 9

Glady Fork, $w$. Va .................... 8

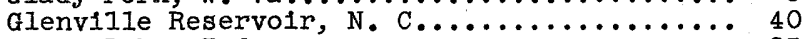

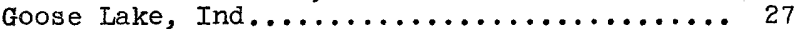

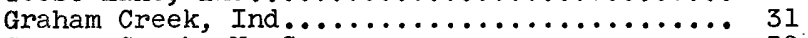

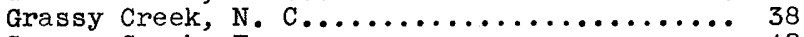

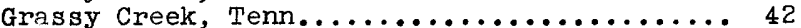

Gready Rock Creek, Tenn.................. 41

Great Falls Lake, Tenn................ 34

Great Falls Reservolr, Tenn................. 34

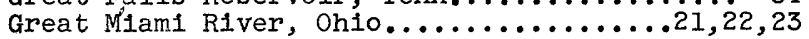

Great Valley Creek, N. Y............... 4

Green Lick Run, Pa.................. 9

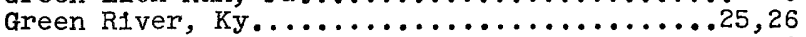

Green River, Tenn.................47-48

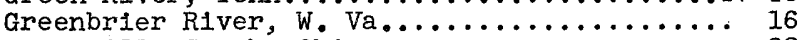

Greenvilie Creek, Ohio................ 22

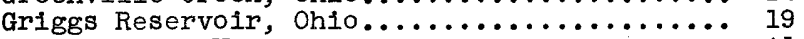

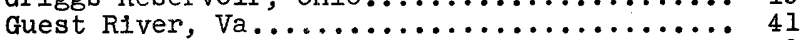

Gum Hollow Branch, Tenn............... 42

Guntersville Lake, Ala ............... 44

Guntersville Reservolr, Ala.................. 44

Guyandot RIver, W. Va................ 18

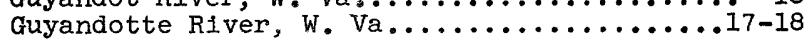

Hackers Creek, w. Va................ 7

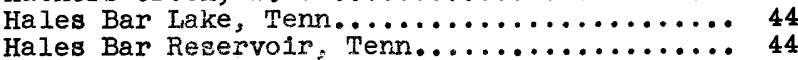


Hardin Creek, Tenn............... Page

Hare Creek, $\mathrm{Pa} . \ldots \ldots \ldots \ldots \ldots \ldots \ldots \ldots \ldots \ldots$

Harpeth River, Tenn.

Harrison Spring, Ind

Hawks Lake, Ind.

Hayes Creek, III.

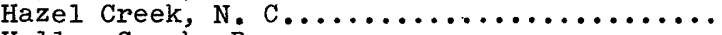

Hellow Creek, $\mathrm{Pa} . \ldots \ldots \ldots \ldots \ldots \ldots \ldots \ldots \ldots \ldots$

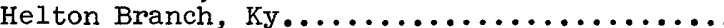

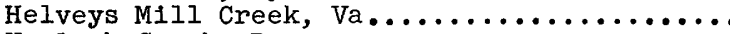

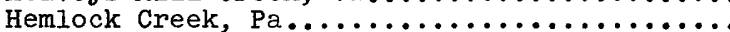

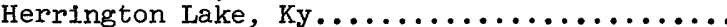

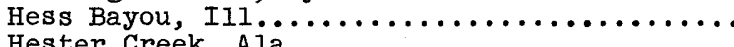

Hickory Creek, Tenn. (tributary to Barren

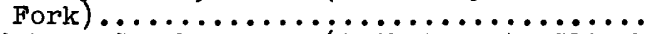

Hickory Creek, Tenn. (tributary to Clinch

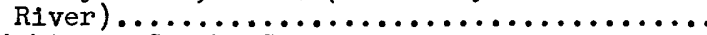

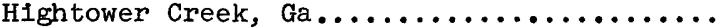

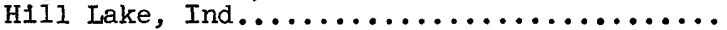

Hinkle Creek, Ind

Hinkley Creek, Ohio

Hiwassee River, Ga.-N. C.-Tenn...........42,

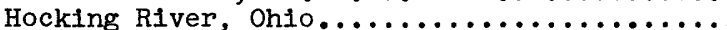

Hodges Bayou, IIl

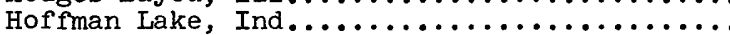

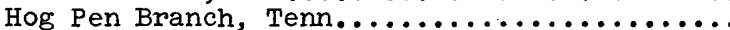

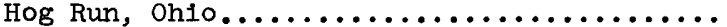

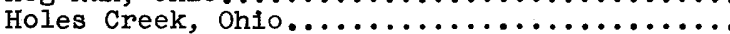

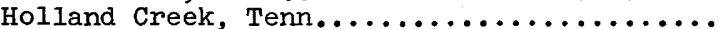

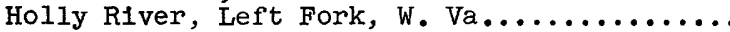

Holston River, Tenn.

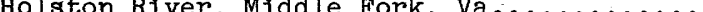

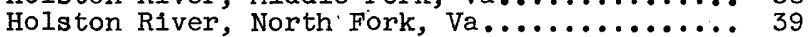

Holston River, South Fork, Tenn.-Va.........37, 38,39

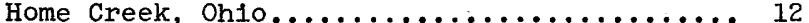

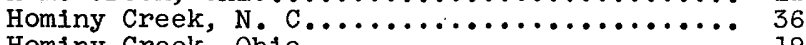

Hominy Creek, Ohio................... 19

Honey Creek, Ind.

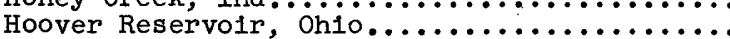

Horse Creek, Il1.

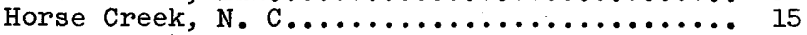

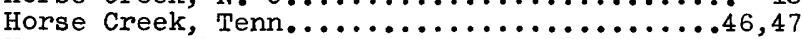

Horseshoe Lake, Ind................. 27

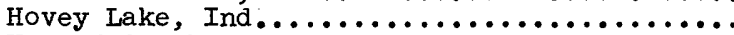

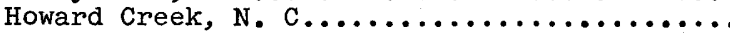

Howard Creek, W. Va

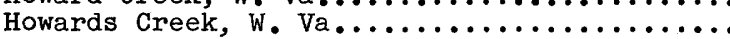

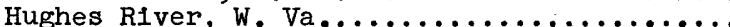

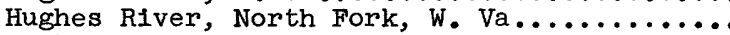

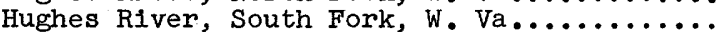

Hunters Run, Ohio.

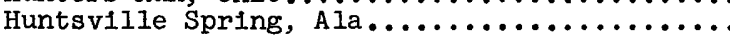

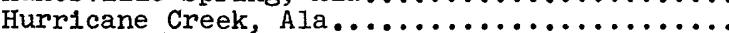

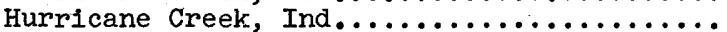

Hurricane Creek, Tenn. (tributary to Duck

River).

Hurricane Creek, Tenn. (tributary to Eik River).

Hurrtcane Creek, w. Va.................

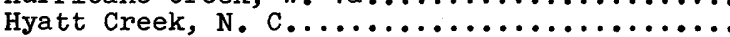

Indian Creek, Ala

Indian Creek, Ind. (tributary to East Fork

White River)

Indian Creek, Ind. (tributary to Faii $\ddot{\text { creekj }}$

Indian Creek, Ind. tributary to Ohio River).

Indian Creek, Ind. (tributary to white

River)...

Indian Creek, ohio (tributary to Great

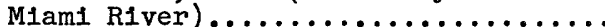

Indian creek, ohio (tributary to scioto River)

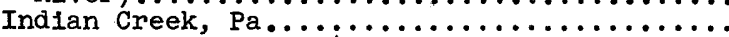

Indian Creek, Tenn. (tributary to Poplar Creek)

Indian Creek, Tenn. (tributary to Tennessee River)

Indian Creek, W. Va................. 16
Indian Fork, ohio................. Pag

Indian Kentuck Creek, Ind................ 24

Indian Lake, Ohio................. 21

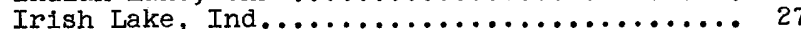

Island Creek, Tenn................... 40

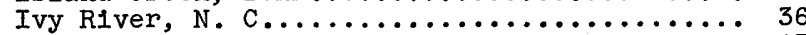

Ivylog Creek, Ga.................. 43

Jackson Run, $\mathrm{Pa} \ldots \ldots \ldots \ldots \ldots \ldots \ldots \ldots \ldots$

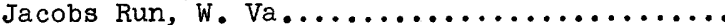

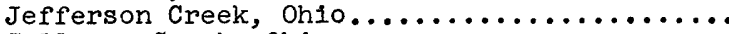

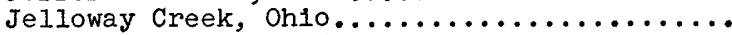

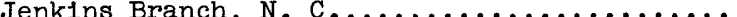

Jerome Fork, Oh10.................. 13

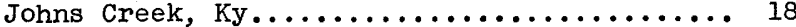

Johnson Lake, Ind .................. 27

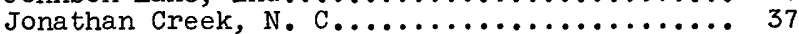

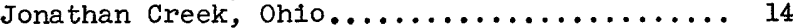

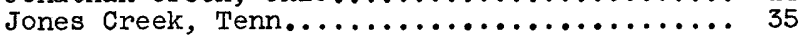

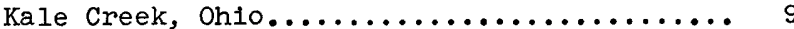

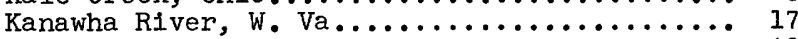

Kentucky Lake, Ky................... 48

Kentucky Reservo1r, Ky............... 48

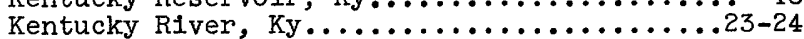

Kentucky River, Middle Fork, Ky........... 23

Kentucky River, North Fork, Ky.......... 23

Kentucky River, South Fork, Ky............ 23

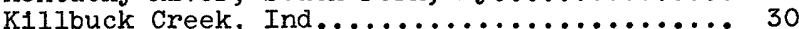

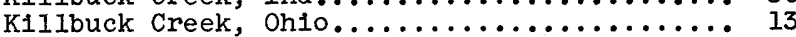

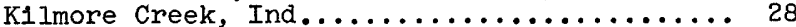

Kinnıkinnick Creek, Ohio.............. 19

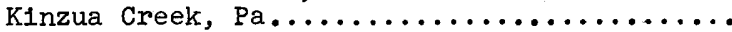

Kiskiminetas River, $\mathrm{Pa} \ldots \ldots \ldots \ldots \ldots \ldots \ldots$

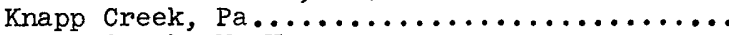

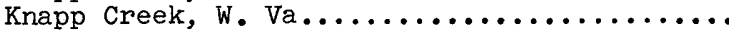

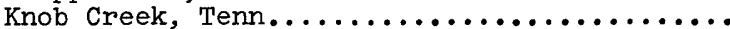

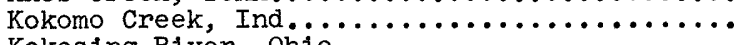

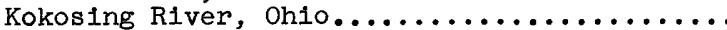

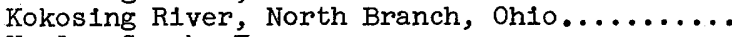

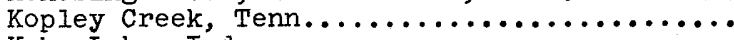

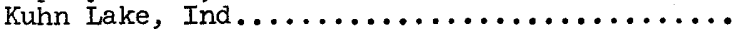

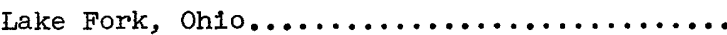

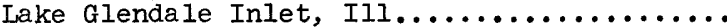

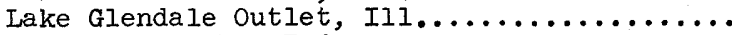

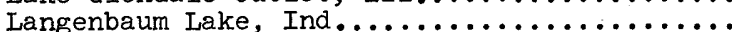

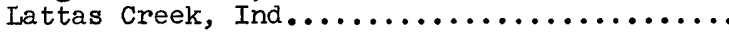

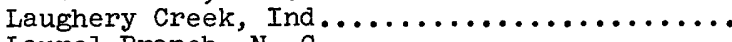

Laurel Branch, N. C. Laurel Fork, w. Va. (tributary to ciear Forkj)

Laurel Fork, W. Va. (tributary to Clear Fork)

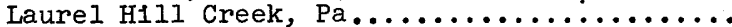

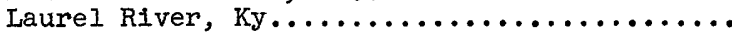

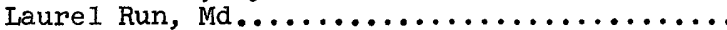

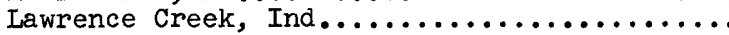

Leading Creek, on1o... (tributary to ilitie

Lanawha River).........................

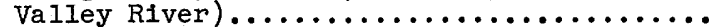

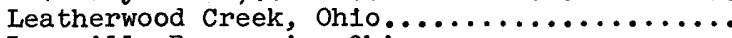

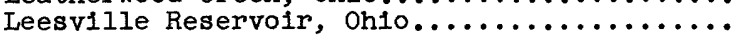

Left Branch or Fork. See name of main stream.

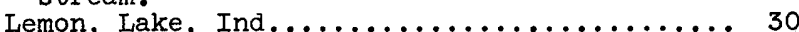

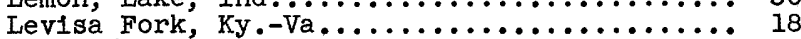

Lew1sburg Lake, Tenn............... 47

Lick Creek, Ind. (tributary to Iost River)... 32

Lick Creek, Ind. (tributary to Mississinewa River).

Lick Creek, Tenn. (tributary to Duck River). Lick Creek, Tenn. (tributary to Nolichucky

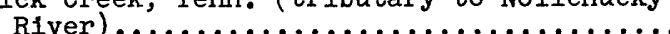

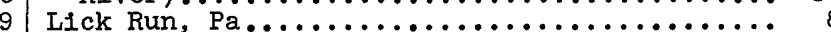

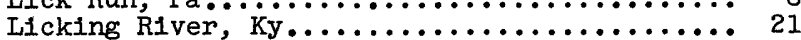

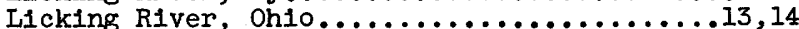

Licking River, North Fork, Ky............ 21

Licking River, North Fork,

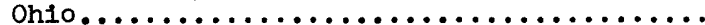
14 
Licking River, North Fork, seepage

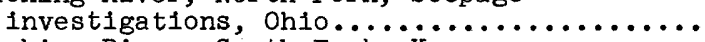

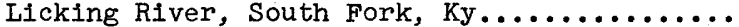

Licking River, South Fork, Ohio............

Licking River basin and adjacent areas low-

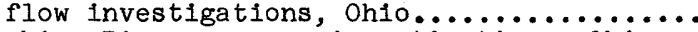

Licking River seepage investigations, Ohio...

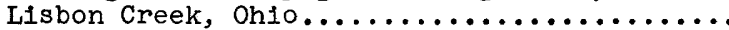

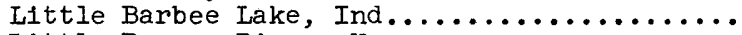

Little Barren River, Ky.

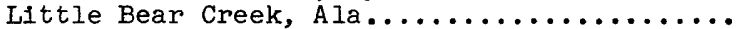

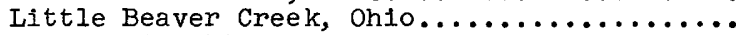

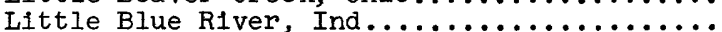

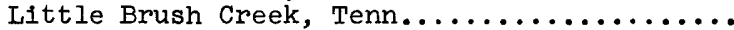

Little Buck Creek, Ind...

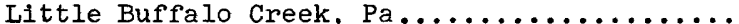

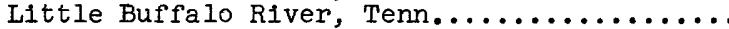

Little Chapman Lake, Ind.

Little Chartiers Creek, $\mathrm{Pa} . . . \ldots \ldots \ldots \ldots \ldots \ldots$

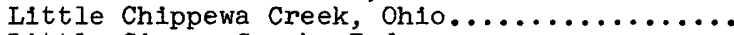

Little Cicero Creek, Ind.

Little Coa 1 River, $W . V a . . . \ldots \ldots \ldots \ldots \ldots \ldots$

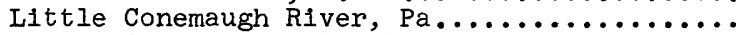

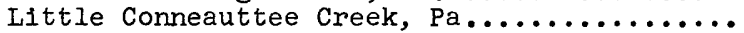

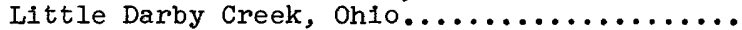

Iittle Duck River, Tenn.

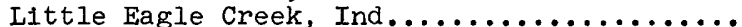

Little Flat Creek, Tenn.

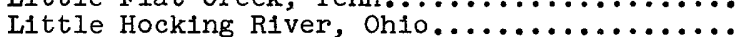

Little Indian Creek, Ind. (tributary to

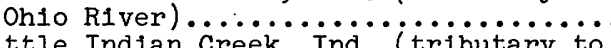

Little Indian Creek, Ind. (tributary to

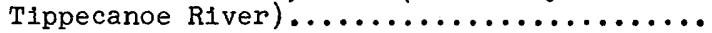

Ilttle Kanawa Rlver, $\mathrm{w} . \mathrm{V} \ldots \ldots \ldots \ldots \ldots \ldots \ldots \ldots \ldots$

Little Kanawa River, West Fork, W. Va.........

I1ttle Mahoning Creek, $\mathrm{Pa} . \ldots \ldots \ldots \ldots \ldots \ldots \ldots$

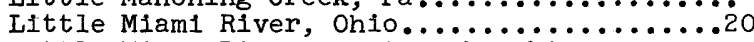

Little Miami River, East Fork, Ohio.........

Little Miami River, North Fork, Oh10.........

Little Miami River basin low-flow

investigations, OhIo

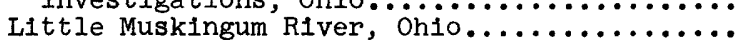

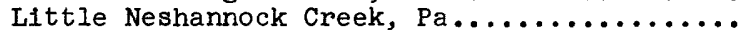

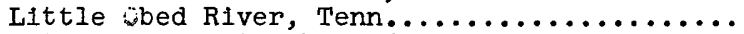

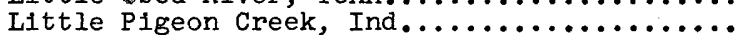

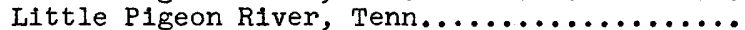

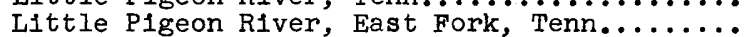

Little Pigeon River, West Fork, Tenn.........

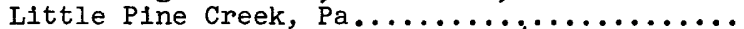

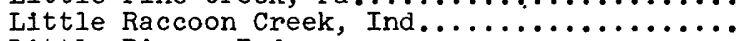

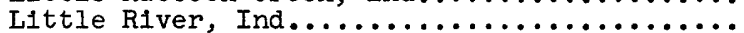

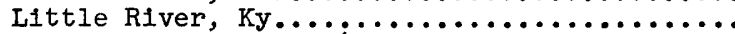

Little River, N. C. (tributary to French

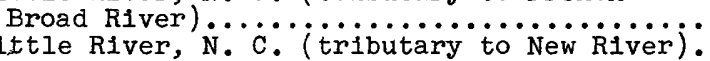

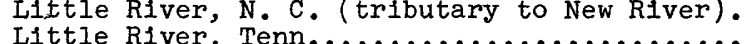

Little River, Va. (tributary to Claytor

Reservolr) ........................

Little River, Va. (tributary to Clinch River)

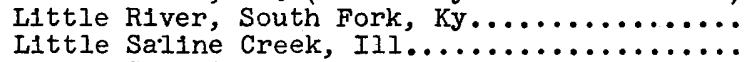

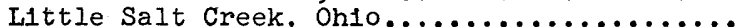

Little Salt Creek, South Branch, Ohlo........

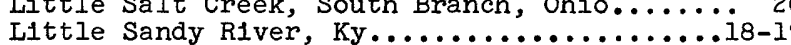

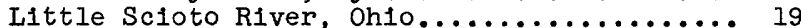

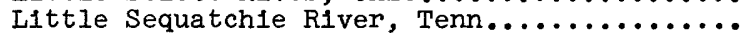

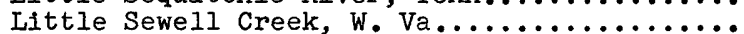

Little Shenango River, $\mathrm{Pa} . . . \ldots \ldots \ldots \ldots \ldots \ldots . . . . .$.

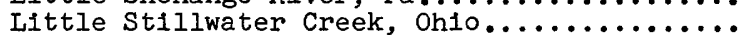

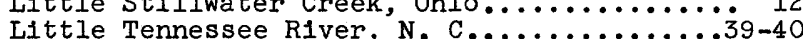

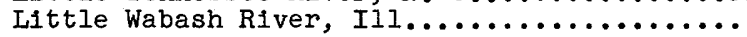

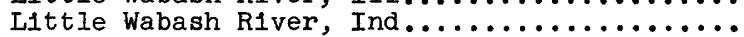

Little Wilson Lake, Ind.................

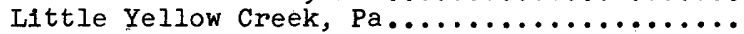

Little Yellow Creek Drainage Canai, Miss......

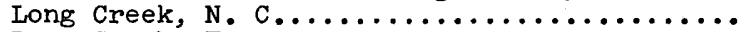

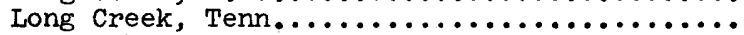

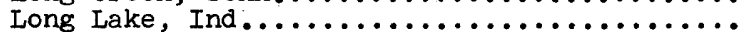

Lookout Creek, Ga....................

Loon Lake, Ind. (tributary to Big Lakej......
Page,

13

13

14

14

\begin{tabular}{l}
27 \\
25 \\
\hline
\end{tabular}

46

31

44
30
7

7
47

27

12
30

30
17
Loon Lake, Ind. (tributary to Tippecanoe

Page

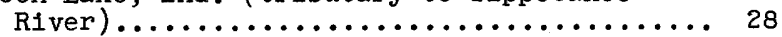

Loop Creek, W. Va...................... 17

Loramie Creek, ohio.................21-22

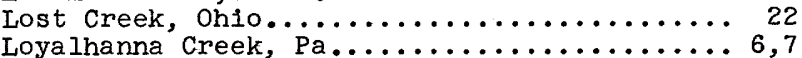

Loya Ihanna Creek Reservoir, $\mathrm{Pa} \ldots \ldots \ldots \ldots \ldots \ldots, 6 \ldots \ldots, 6 \ldots \ldots$

Lukens Lake, Ind.................. 26

Lynn Camp Creek, Ky.................... 33

McClures Lake, Ind................... 28

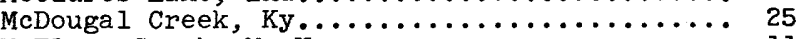

McElroy Creek, w. Va................ 11

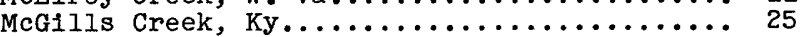

McGinnis Run, $\mathrm{Pa} \ldots \ldots \ldots \ldots \ldots \ldots \ldots \ldots \ldots, 6 \ldots \ldots$

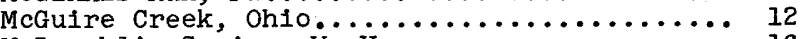

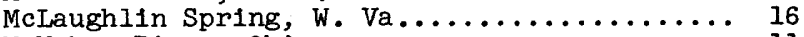

McMahon River, ohio................. 11

McWlll1ams Creek, Tenn................ 44

Mad River, Ohio................... 22

Mad River basin and adjacent areas low-

flow investigations, ohio.............. 22

Mad River seepage investigation, ohio......... 22

Madden Creek, Ill..................... 32

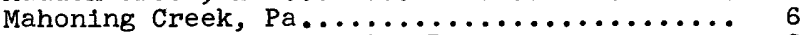

Mahoning Creek Reservoir, $\mathrm{Pa} \ldots \ldots \ldots \ldots \ldots \ldots \ldots, 6 \ldots \ldots \ldots, 6 \ldots \ldots,{ }_{6}$

Mahoning River, ohio $\ldots \ldots \ldots \ldots \ldots \ldots \ldots, 10$

Mahoning River, West Branch, Ohio...........

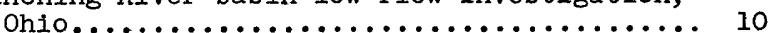

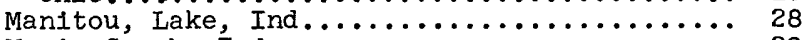

Maria Creek, Ind.................... 29

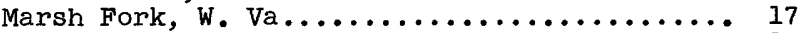

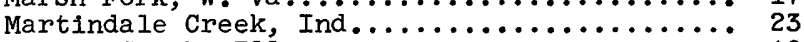

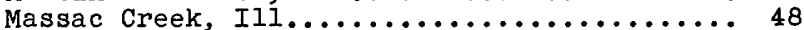

Massie Creek, Oh10.................... 20

Massle Creek, North Fork, ohio.............. 20

Massie Creek, South Fork, Oh10........... 20

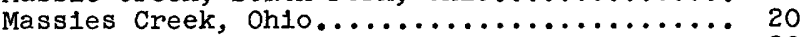

Massies Creek, North Fork, Oh10...........20

Massies Creek, South Fork, Oh10.............. 20

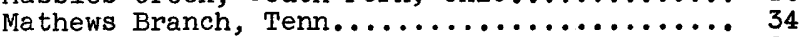

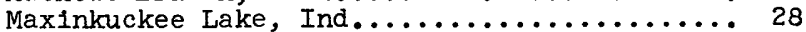

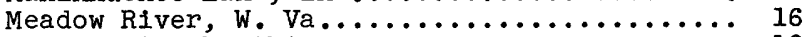

Meander Creek, Ohio.................. 10

Meander Creek Reservolr, Oh10.............. 10

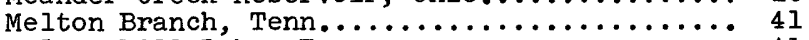

Melton Hill Lake, Tenn............... 41

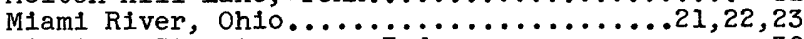

M1chlgan street sewer, Ind............... 30

Middle Branch or Fork. See name of main

stream.

Middle Creek, $P a \ldots \ldots \ldots \ldots \ldots \ldots \ldots \ldots \ldots$

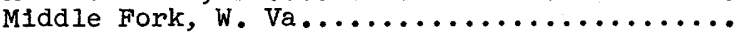

Middle Island Creek, $W$. Va............. II

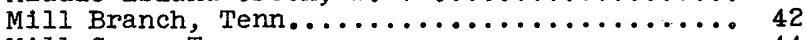

Mill Cave, Tenn................... 44

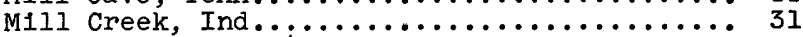

Mill Creek, oh1o (tributary to Mahoning

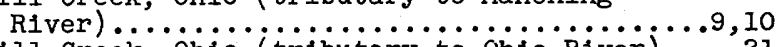

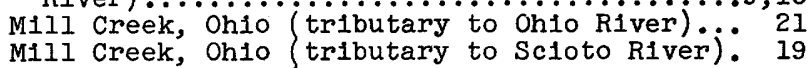

Mill Creek, Ohio (tributary to Walhonding

River)...................................

li crek, Pa. (tributary to Loyalhanna

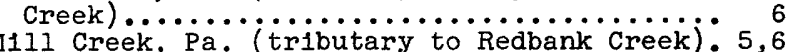

Mill Creek, Tenn. (tributary to Cumberland

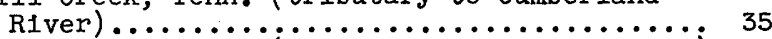

Mill Creek, Tenn. (tributary to öcöe River) 43

Mill Creek, Va..................... 15

Mill Creek, w. Va. (tributary to New River) 16

M1ll Creek, W. Va. (tributary to onlo River). 15

Mill Creek, West Fork, Oh10............. 21

Mill Creek Reservolr, West Fork, ohio.......... 21

M11l Spring, Tenn................. 39

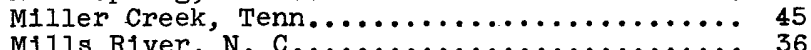

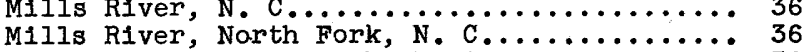

Mills River, South Fork, N. C............... 36 


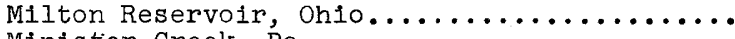

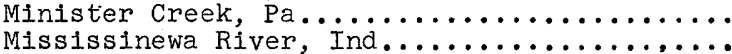

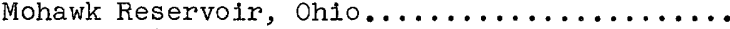

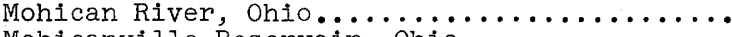

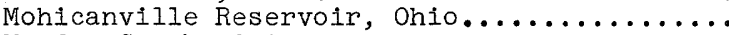

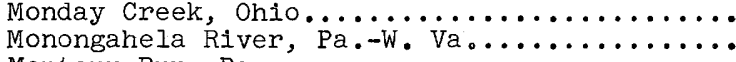

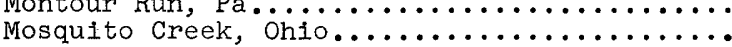
Mosquito Creek Reservoir, ohio.............

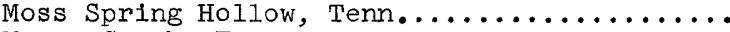

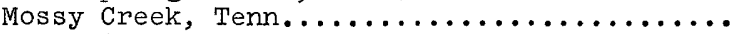

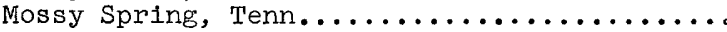

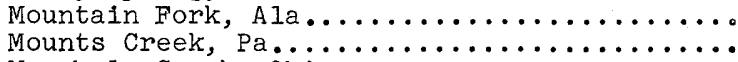

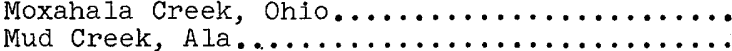
Mud Creek, Ind. (tributary to Faii Creek) .... Mud Creek, Ind. (tributary to Tippecanoe River).

Mud creek, Ind. (tributary to wildcat Creekj) Mud Creek, N. C.................... Mud River, Ky.

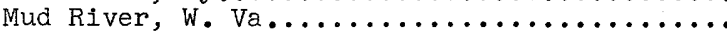

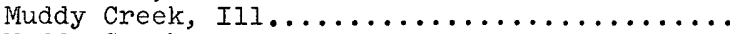

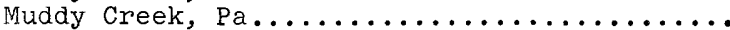

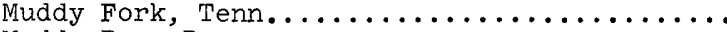
Muddy Run, $\mathrm{Pa} \ldots \ldots \ldots \ldots \ldots \ldots \ldots \ldots \ldots \ldots$

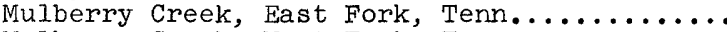

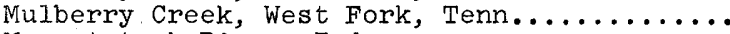

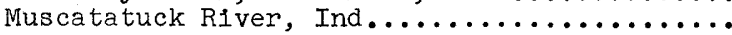

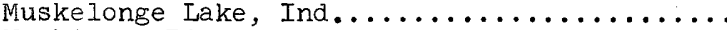

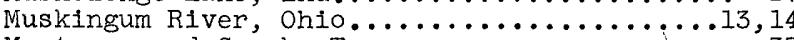

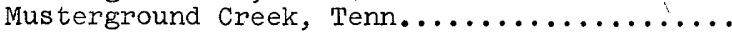

Nails Creek, Tenn.

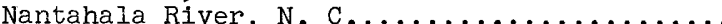

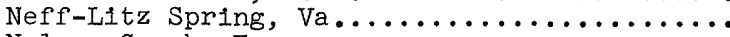

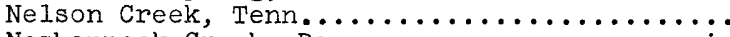

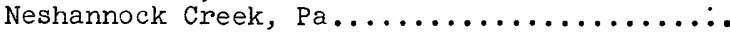

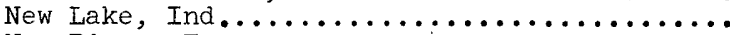

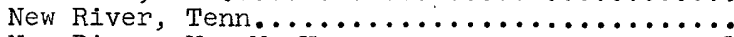

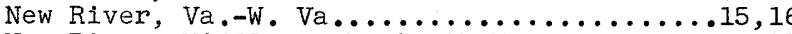
New River, Middle Fork, South Fork, N. C..... 15

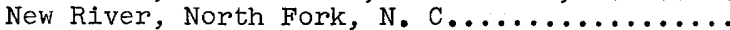

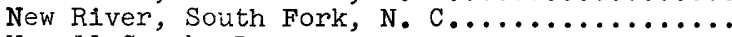

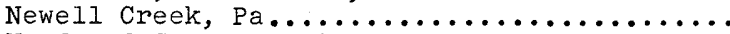

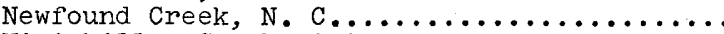

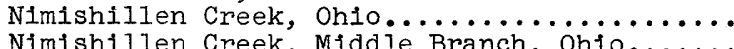

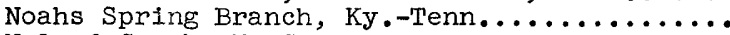

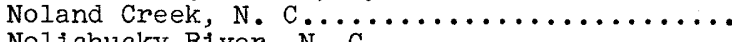

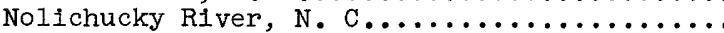

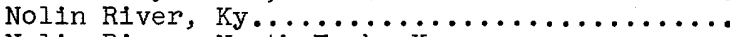

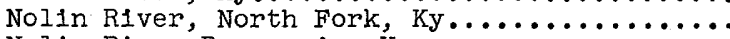

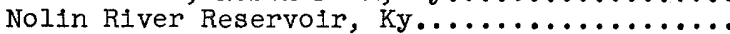

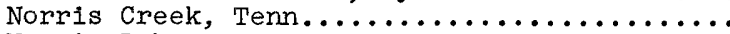
Norris Lake, Tenn.

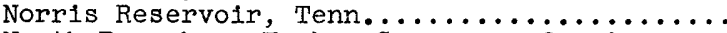
North Branch or Fork. See name of main

stream.

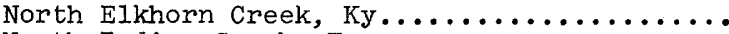

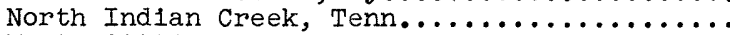

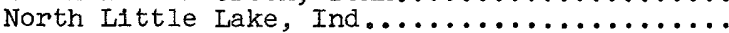

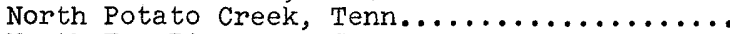

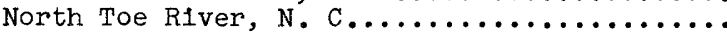
Norwood Creek, Tenn

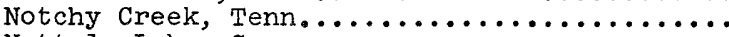
Nottely Lake, Ga.

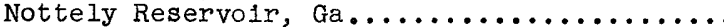

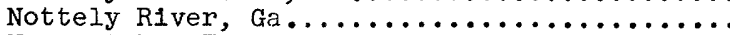

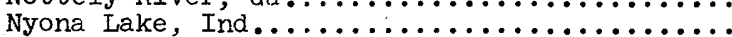

o'Shaughnessy Reservo1r, Oh10.............

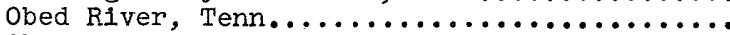

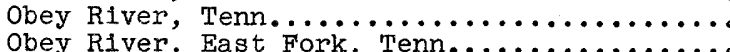

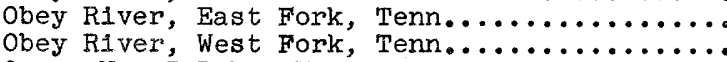

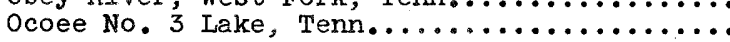

Page
Page 43

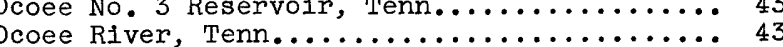

Oconaluftee River, $\mathrm{N} . \mathrm{C} \ldots \ldots \ldots \ldots \ldots \ldots \ldots \ldots \ldots \ldots . \ldots \ldots \ldots \ldots$

Ogle Lake, Ind............................... 32

Ohio Brush Creek, Ohio........

W. Va....9, 11, 14,15,17, 18,20,21, 24, 25, 26,33,48

011 Creek, Pa.................... 5

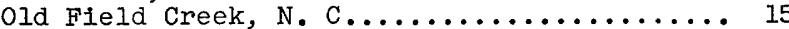

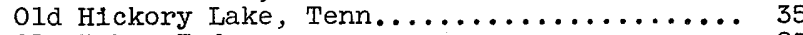

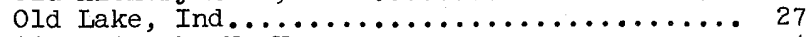

Olean Creek, N. Y.................

Olentangy River, Ohio............... 19

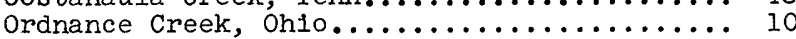

Oswayo Creek, N. Y.-Pa......................

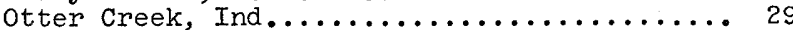

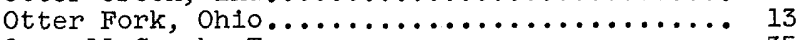

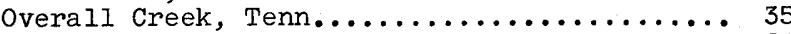

Owen Branch, Tenn.................. 34

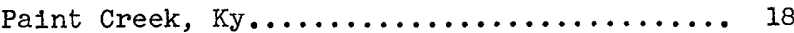

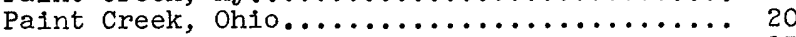

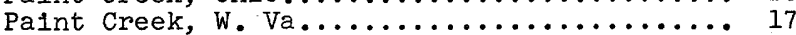

Paint Creek, East Fork, Ohio............. 20

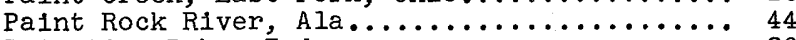

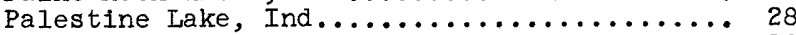

Panther Creek, w. Va................... 18

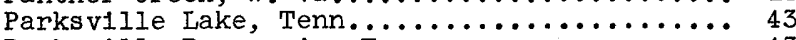

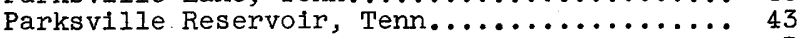

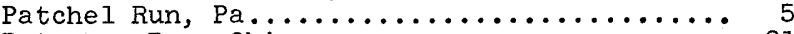

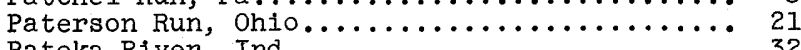

Patoka River, Ind.................. 32

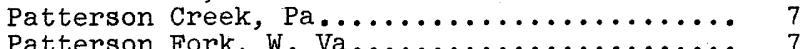

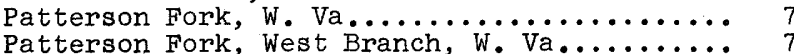

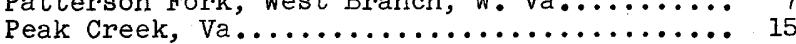

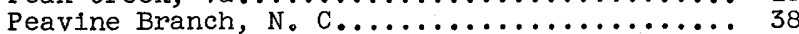

Pennsylvania Fork. See name of main stream.

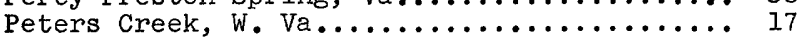

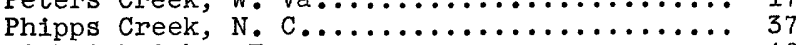

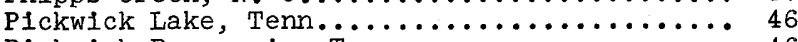

Pickwick Reservolr, Tenn.............. 46

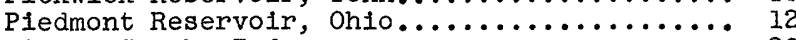

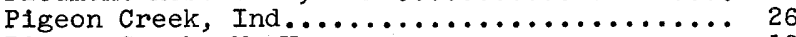

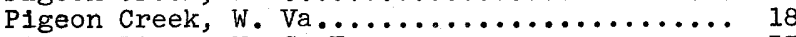

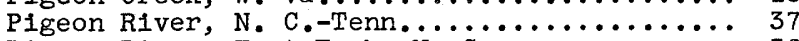

Pigeon River, East Fork, N. C............ 36

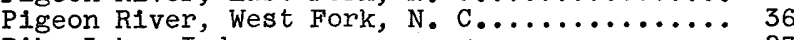

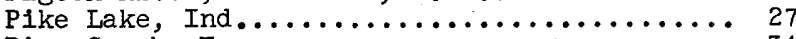

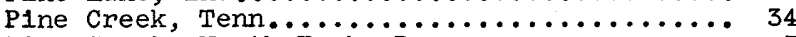

Pine Creek, North Fork, Pa........... 7

Piney Creek, Ala .............................. ${ }^{45}$

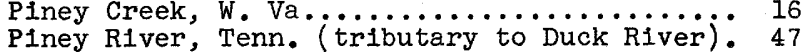

Piney River, Tenn. (tributary to Tennessee

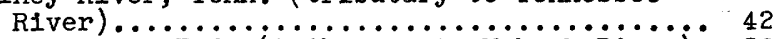

Pipe Creek, Ind. (tributary to wabash River). 26

Pipe Creek, Ind. (tributary to White River).. 30

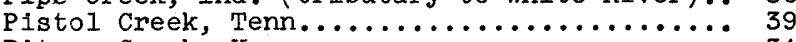

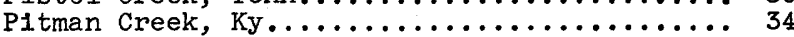

Pleasant H1ll Reservoir, ohio................. 13

Pleasant Run, Ind........................ 30

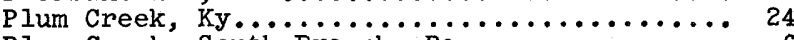

Plum Creek, South Branch, $\mathrm{Pa} . \ldots \ldots \ldots \ldots \ldots$

Plum Creek subwatershed Nos. 4,15 , and

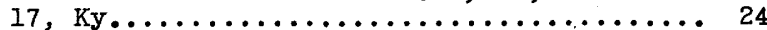

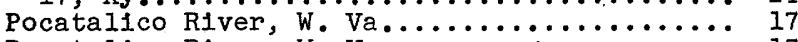

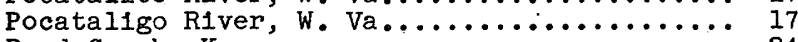

Pond Creek, Ky .................... 24

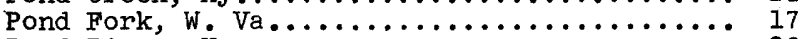

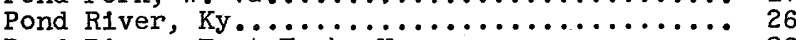

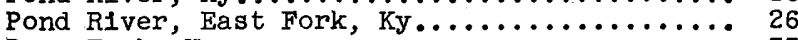

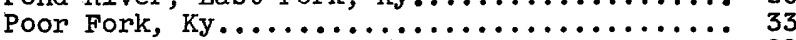

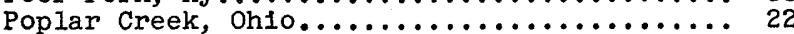

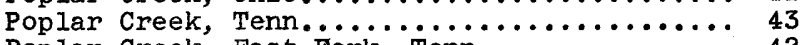

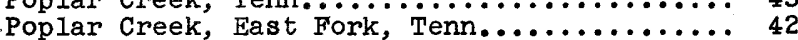

Poplar Run, Pa ........................ 9

Potato Creek, $\mathrm{Pa} \ldots \ldots \ldots \ldots \ldots \ldots \ldots \ldots \ldots \ldots \ldots \ldots$ 


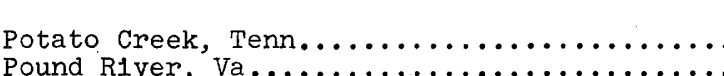

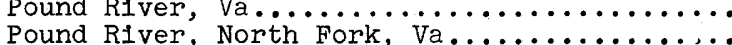

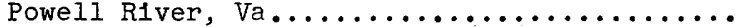

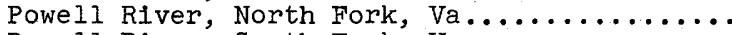

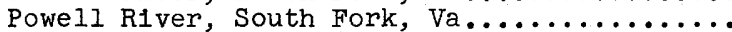

Prairie Creek, Ind. (tributary to Wabash

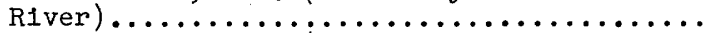

Prairie Creek, Ind. (tributary to White

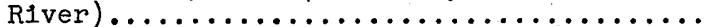

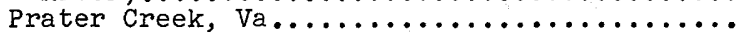

Pryor Cove Branch, Tenn................

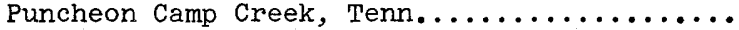

Pymatuning Creek, $\mathrm{Pa} . \ldots \ldots \ldots \ldots \ldots \ldots \ldots \ldots$

Pymatuning Reservoir, $\mathrm{Pa} \ldots \ldots \ldots \ldots \ldots \ldots \ldots$

"Q" ditch, II1.

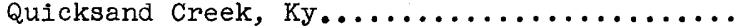

Quililn Spring, $\mathrm{Va} . \ldots \ldots \ldots \ldots \ldots \ldots \ldots \ldots \ldots \ldots$

Raccoon Creek, IIl.-Ind.

Raccoon Creek, Ohıo (tributary to öhio River)

Raccoon Creek, Ohio (tributary to South Fork

Licking River)...

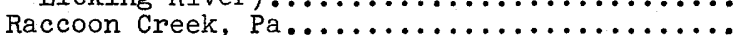

Raccoon Creek seepage investigations, Oh10...

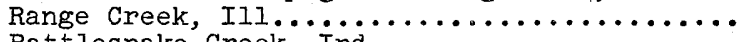

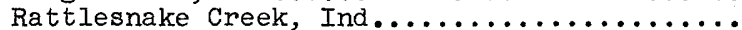

Rattlesnake Creek, Oh10...............

Red River, Ky....

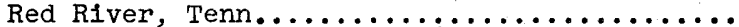

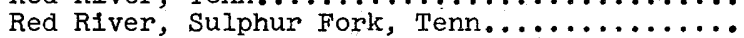

Red River, West Fork, Tenn..............

Redbank Creek, $\mathrm{Pa}$....

Redstone Creek; $\mathrm{Pa}$

Reed Creek, Va.0...

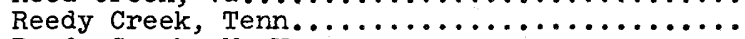

Reedy Creek, W. Va.

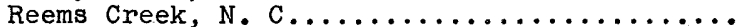

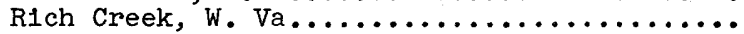

Richey Run, Pa.

Richland Creek, Ind.

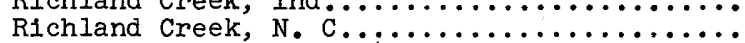

Richland Creek, Tenn. (tributary to Eik

Richland Creek, Tenn. (tributary to

Tennessee River)

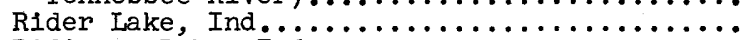

Ridinger Lake, Ind $\ldots \ldots \ldots \ldots \ldots \ldots \ldots \ldots \ldots \ldots$

Roan Creek, Tenn...................

Roaring River, Tenn.................

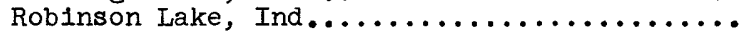

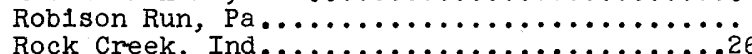

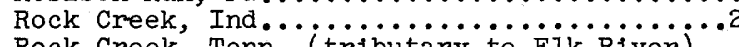

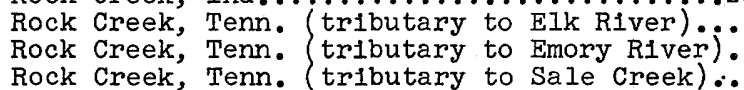

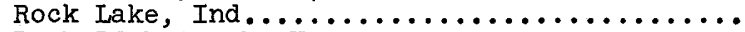

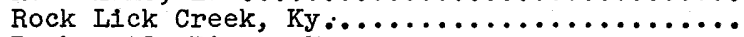

Rockcastle RIver, Ky.

Rocky Fork, Oh1o (tributary to Black Forkj ...

Rocky Fork, Ohio (tributary to Paint Creek)...

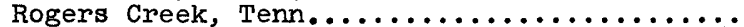

Rolling Fork, Ky $\ldots \ldots \ldots \ldots \ldots \ldots \ldots \ldots \ldots$

Rose Creek, Ky..

Ross Ade drain, Ind.

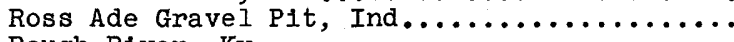

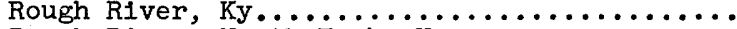

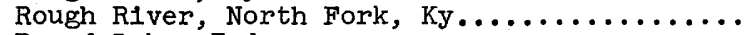

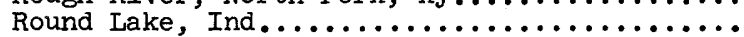

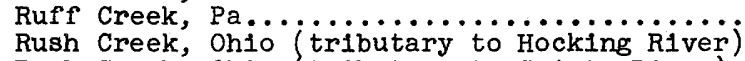

Rush Creek, Ohio (tributary to Scioto River).

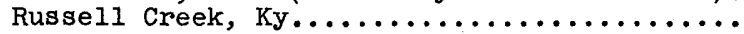

Russell Creek, Tenn.

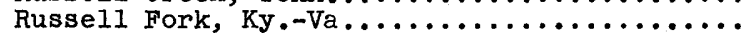

Rutherford Creek, Tenn.
Page
Salamonie River, Ind.............. Page

Salem Fork, $W$. Va................. 7,8

Salem Fork subwatershed Nos. 9 , and $11 \mathrm{~A}$,

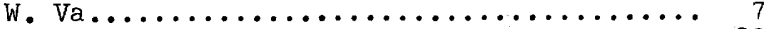

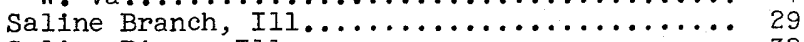

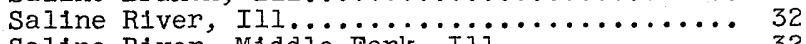

Saline River, Middle Fork, Ill.......... 32

Salt Creek, Ind. (tributary to East Fork

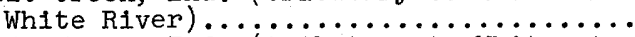

Salt Creek, Ind. (tributary to Whitewater

River) ..................................

River)

Salt Creek, ohio (tributary to Scioto River). ${ }_{20}^{14}$

Salt Creek, Middle Fork, Ind............ 32

Salt Creek, North Fork, Ind............. 32

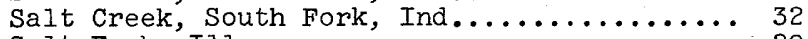

Salt Fork, Ill.................. 29

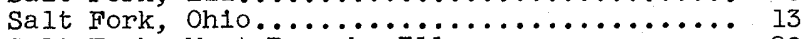

Salt Fork, West Branch, Iii.............. 29

Salt Lick Creek, W. Va.............. 8

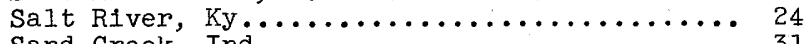

Sand Creek, Ind $\ldots \ldots \ldots \ldots \ldots \ldots \ldots \ldots \ldots \ldots \ldots$

Sand Run, W. Va........................ 7

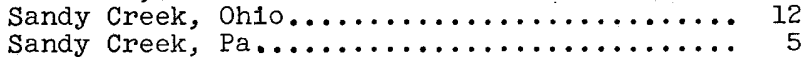

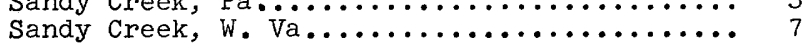

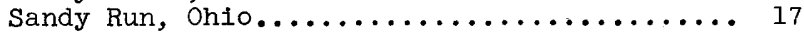

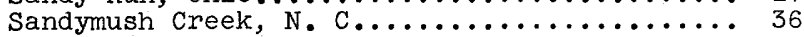

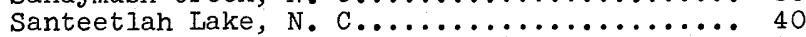

Santeetlah Reservoir, N. C............. 40

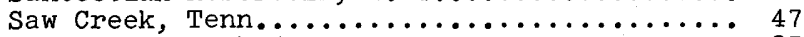

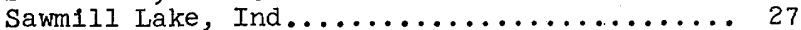

Scarboro Creek, Tenn..................... 41

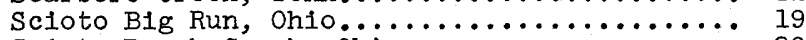

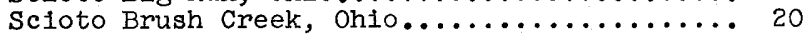

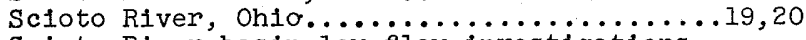

Scioto River basin iow-fiow investigations,

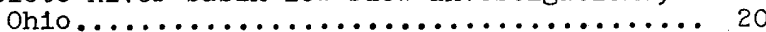

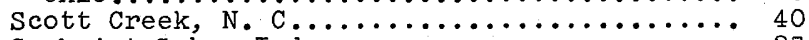

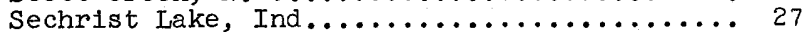

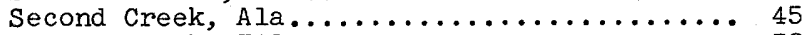

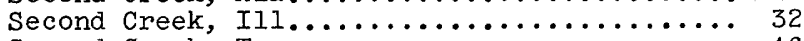

Second Creek, Tenn.................. 46

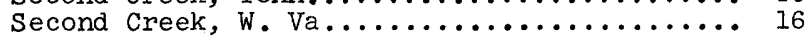

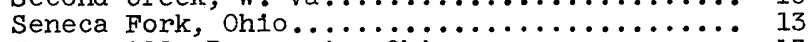

Senecaville Reservoir, Ohio............. 13

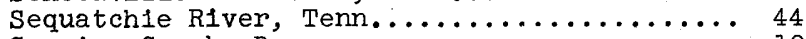

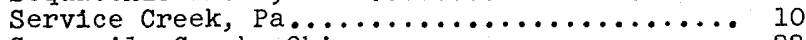

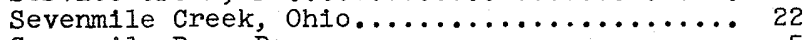

Sevenmile Run, $\mathrm{Pa} \ldots \ldots \ldots \ldots \ldots \ldots \ldots \ldots \ldots \ldots \ldots \ldots$

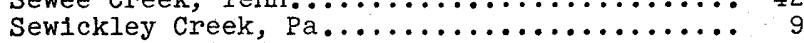

Shade River, ohio......................... 15

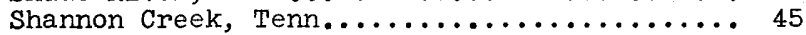

Shavers Fork, W. Va.................... 8

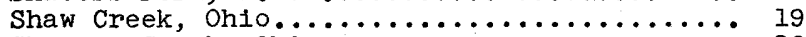

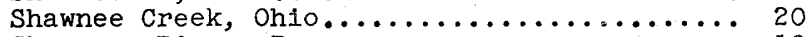

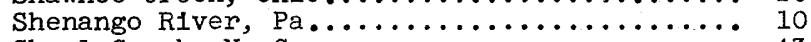

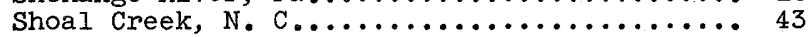

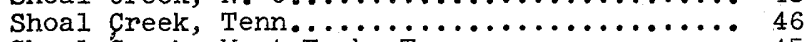

Shoa1 Creek, West Fork, Tenn................. 45

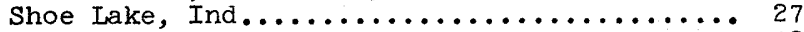

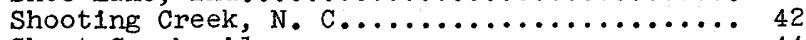

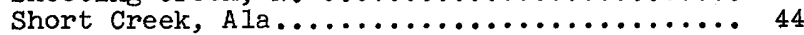

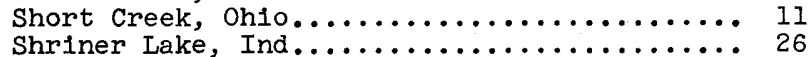

S1lver Creek, Ind. (tributary to East Fork

Whitewater River) ................ 23 Silver Creek, Ind. (tributary to ohio River). 24

Silver Lake, Ind.................. 26

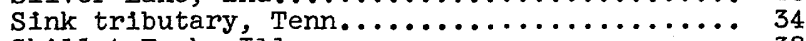

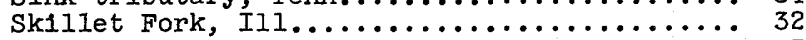

Skin Creek, ${ }_{\text {S }}$. Va $\ldots \ldots \ldots \ldots \ldots \ldots \ldots \ldots \ldots \ldots \ldots \ldots{ }_{10}{ }^{7}$

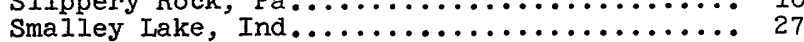

Snowbird Creek, N. C................. 40 
Soddy Creek, Tenn.

South Branch or Fork. See name of main stream.

South Chestuee Creek, Tenn.

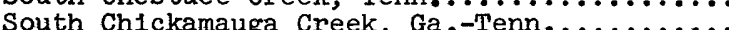

South Elkhorn Creek, Ky................

South Hogan Creek, Ind...

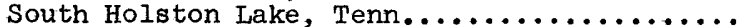

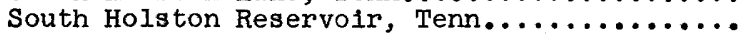

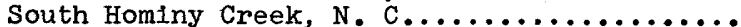

South Mouse Creek, Tenn

South Mud Lake, Ind....

South Sauty Creek, Ala.

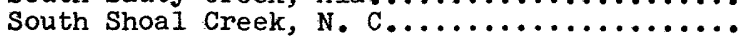

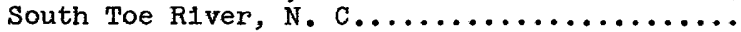

Spencer Creek, Tenn...................

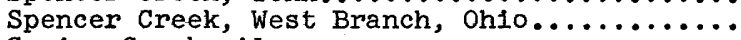

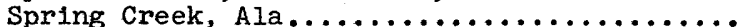

Spring Creek, Ind...................

Spring Creek, N. C.

Spring Creek, Tenn. (tributary to Cumberland

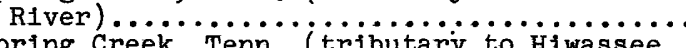

Spring Creek, Tenn. (tributary to Hiwassee

River) ............ (tributary to West Fork

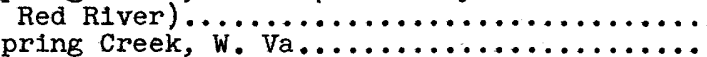

Spring Creek, w. Va $\ldots \ldots \ldots \ldots \ldots \ldots \ldots \ldots \ldots \ldots \ldots$

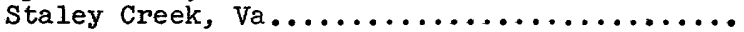

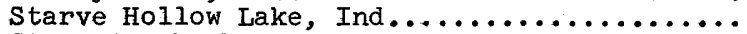

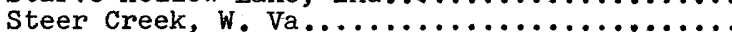

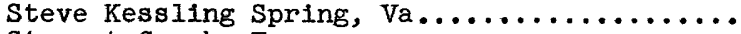

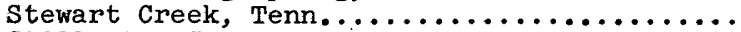

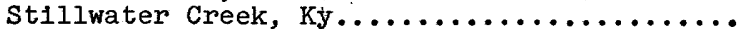

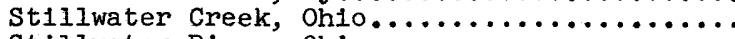

Stillwater River, Ohio.

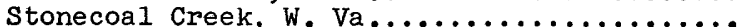

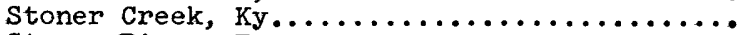

Stones River, Tenn...................

Stones River, East Fork, Tenn............

Stoney Creek, Ind ....................

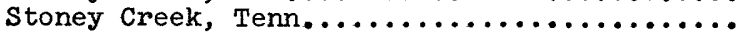

Stony Creek, $\mathrm{Pa} \ldots \ldots \ldots \ldots \ldots \ldots \ldots \ldots \ldots \ldots \ldots$

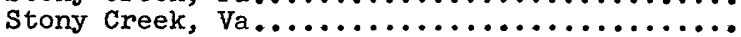

Stucker Creek, Ind...................

Stucker Fork, Ind $\ldots \ldots \ldots \ldots \ldots \ldots \ldots \ldots \ldots \ldots$

Stump Creek, $\mathrm{Pa} . \ldots \ldots \ldots \ldots \ldots \ldots \ldots \ldots \ldots \ldots \ldots \ldots . . \ldots \ldots$

Suee Creek, Tenn....................

Sugar Creek, Ala

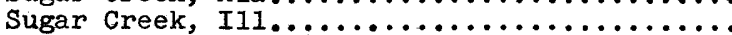

Sugar Creek, Ind. (tributary to Big Blue

River)...

Sugar Creek, Ind. (tributary to wabash River)

Sugar Creek, Oh10...................

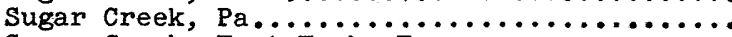

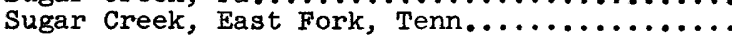

Sugar Creek, West Branch, $\mathrm{Pa} \ldots \ldots \ldots \ldots \ldots \ldots \ldots$

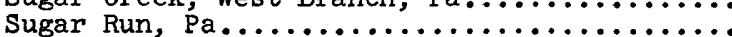

Sulphur Fork. See name of main stream.

Sunday Creek, ohlo.

Sunfish Creek, Ohio (tributary to onio Riverj

Sunfish Creek, Ohio tributary to Scioto River)...

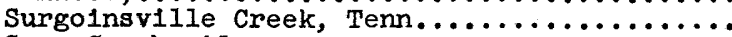

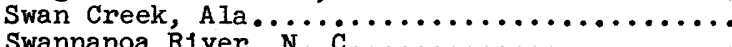

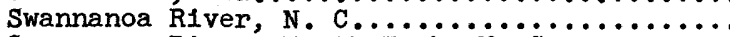

Swannanoa River, North Fork, $\mathrm{N} . \mathrm{C} \ldots \ldots \ldots \ldots \ldots$

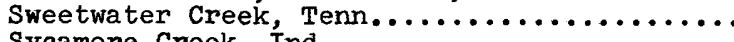

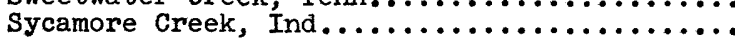

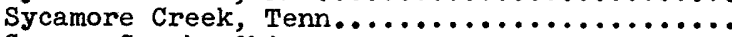

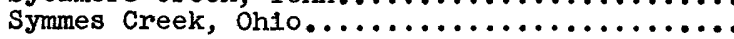

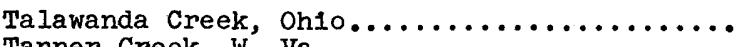

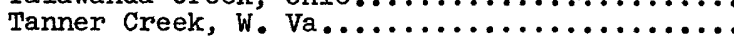

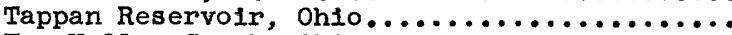

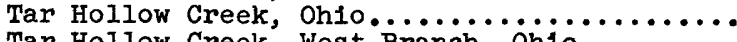

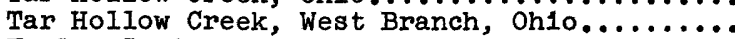

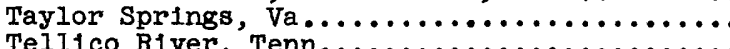

T $T$

Ten Mile Creek, Tenn................ 42

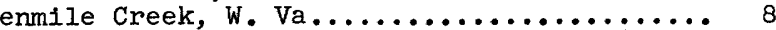

Tennessee River, Ala.-

Tenn................39,40,42,44,45,46,47,48

Thompson Creek, va.................. 41

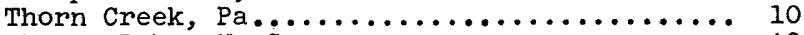

Thorpe Lake, N. C..................... 40

Threefork Creek, $w . V a \ldots \ldots \ldots \ldots \ldots \ldots \ldots \ldots$

Timber Run, Oh10................... 14

Tionesta Creek, $\mathrm{Pa} . \ldots \ldots \ldots \ldots \ldots \ldots \ldots . . \ldots$ 4-5

Tionesta Creek, South Branch, $\mathrm{Pa} . \ldots \ldots \ldots \ldots{ }_{4}^{4}$

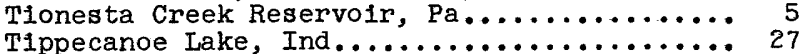

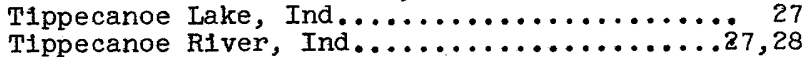

Toccoa River, Ga......................... 43

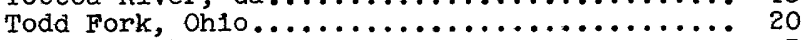

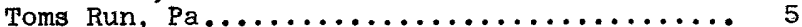

Touby Run, Ohio........................ 13

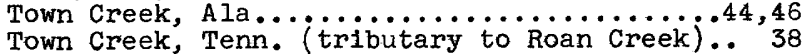

Town Creek, Tenn. (tributary to Whiteoak

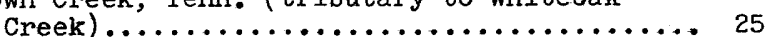

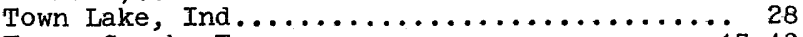

Trace Creek, Tenn................... 47,4

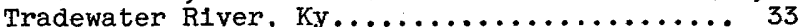

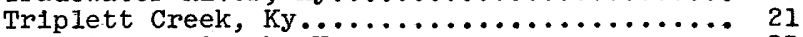

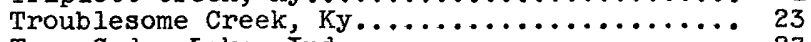

Troy Cedar Lake, Ind................. 27

Tuckasegee RIver, N. C................. 40

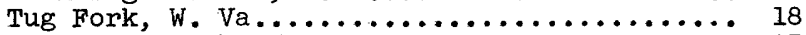

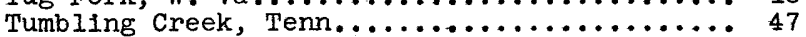

Tunungwant Creek, East Branch, Pa............. 4

Turkey Creek, Ala.................... 46

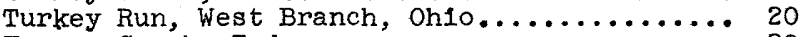

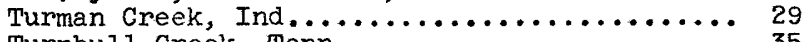

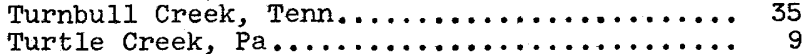

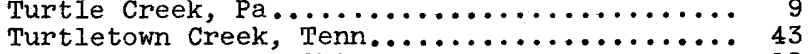

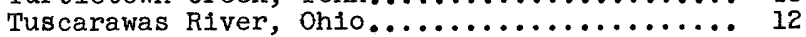

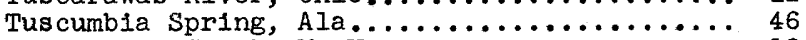

Twelvepole Creek, w. Va................ 18

Twelvepole Creek, East Fork, W. Va......... 18

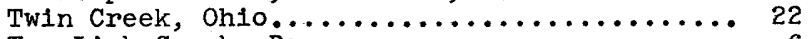

Two Lick Creek, $\mathrm{Pa} . \ldots \ldots \ldots \ldots \ldots \ldots \ldots \ldots \ldots$

Tygart Lake, $W$. Va......................... 7

Tygart Reservoir, W. Va.................. 7

Tygart Valley River, $W$. Va............. 7

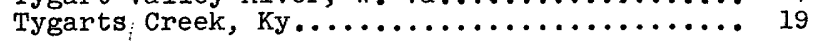

Upper Twin Creek, Oh1o............... 20

Valley River, N. C.................. 43

Van Horne Creek, Pa...................... 5

Varner Hollow Run, w. Va................. 7

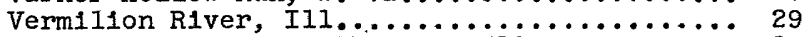

Vermilion River, North Fork, Iij.......... 29

Vermilion River, Salt Fork, Ill........... 29

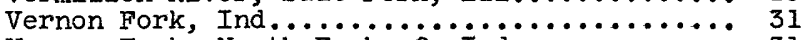

Vernon Fork, North Fork of, Ind ............. 31

Versa1lles Lake, Ind................. 23

Wabash River, IIl.-Ind...........26,27, 29, 32

Wabash Spring, Va.................. 15

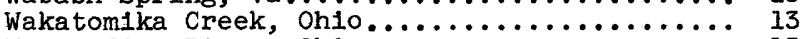

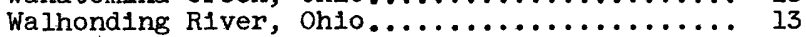

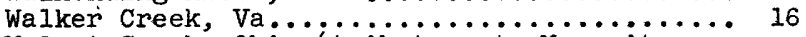

Walnut Creek, Ohio (tributary to Mosquito

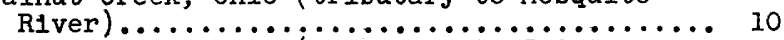

Walnut creek, ohio (tributary to scioto

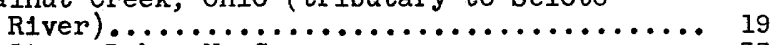

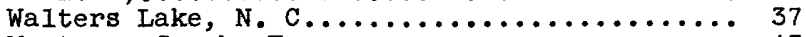

Wartrace Creek, Tenn..................... 47

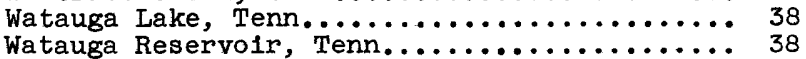

Wa tauga Reservo1r, Tenn .......................

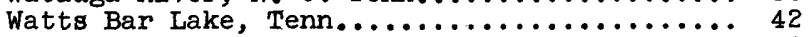

Watts Bar Reservoir, Tenn............... 42

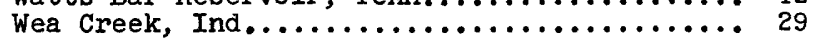


Weakley Creek, Tenn.............. 45

Weakly Creek, Tenn............................ 47

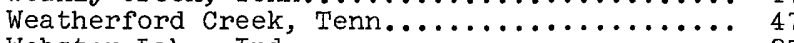

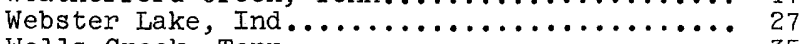

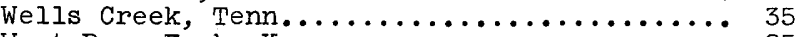

West Bays Fork, Ky................... 25

West Branch or Fork. See name of main stream.

West Ch1ckamauga Creek, Ga............ 44

West Flint Creek, Ala.................... 45

West Fork River, W. Va.............. 7,8

West Harpeth River, Tenn............... 35

West Sandy Creek, Tenn............... 48

Wheeler Lake, Ala ................. 45

Wheeler Reservoir, Ala ................. 45

Wheeling Creek, ohio-W. Va.............. II

Wheeling Creek, North Fork, Dunkard Fork, Pa. II
Wheeling Creek, South Fork, Dunkard Fork, Pa. 11

Whetstone Creek, Ohio (tributary to Black

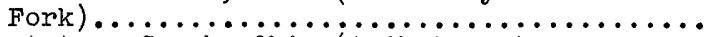

Whetstone creek, ohio (tributary to

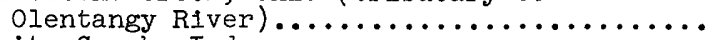

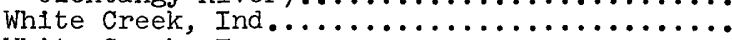

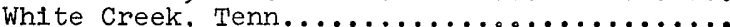

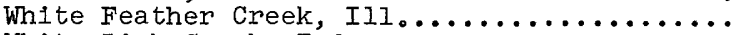

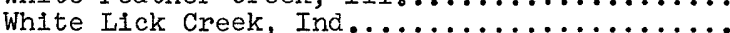

White Lick Creek, West Fork, Ind........... 30

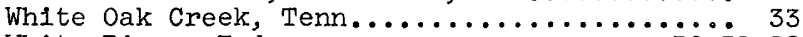

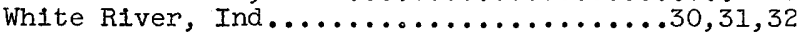

White River, East Branch, Ind............. 32

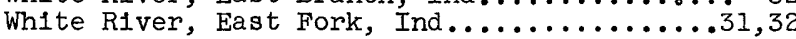

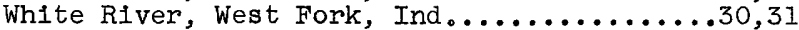

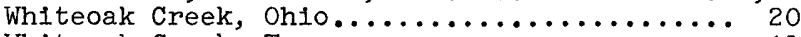

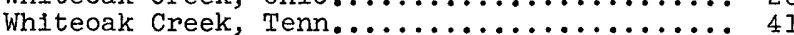

Whites Creek, Tenn. (tributary to Cumberland

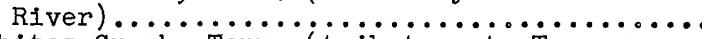

Whites Creek, Tenn. (tributary to Tennessee

River).......................
Whites Run, Pa................. Page

Whitewater River, Ind............... 23

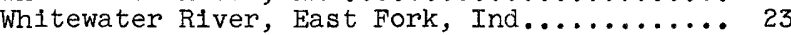

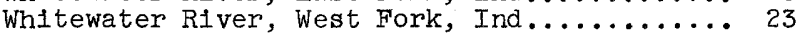

Wildcat Creek, Ind................... 28

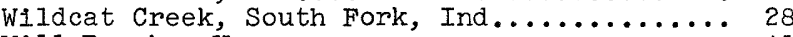

Will Brooks, $V a . \ldots \ldots \ldots \ldots \ldots \ldots \ldots \ldots \ldots \ldots . \ldots . \ldots 1$

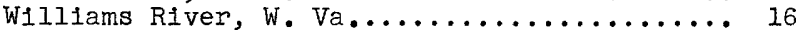

Willow Creek, onio......................... 9

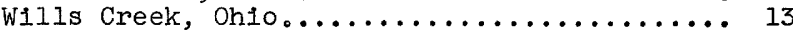

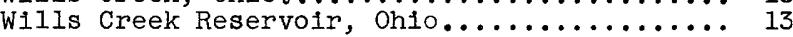

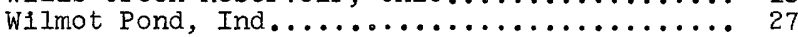

Wilson Lake, Ala .................... 46

Wilson Lake, Ind................. 26

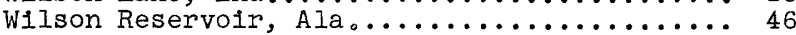

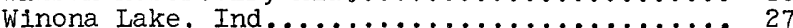

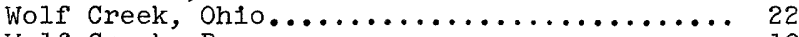

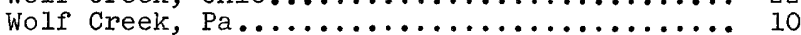

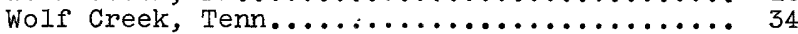

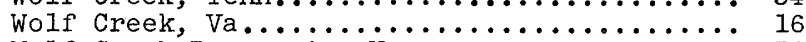

Wolf Creek Reservolr, Ky............... 34

Wolf River, Tenn...................... 34

Woods Reservolr, Tenn.......................... 45

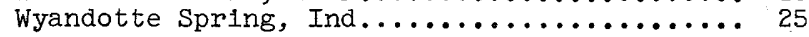

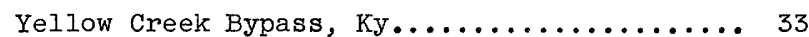

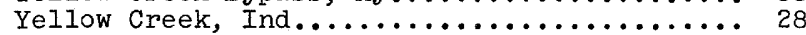

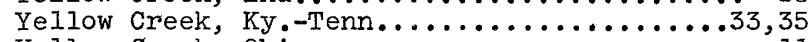

Yellow Creek, ohio........................ 11

Yellow Creek, North Fork, Ohio............. Il

Yellow Creek Drainage Canal,

Miss.......................... 46

Youghiogheny River, Md. $-\mathrm{Pa} \ldots \ldots \ldots \ldots \ldots \ldots \ldots, 8,9$

Youghiogheny River Reservolr, $\mathrm{Pa} \ldots \ldots \ldots \ldots \ldots 9 . \ldots 9$

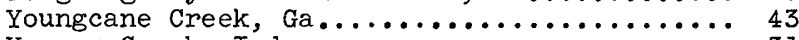

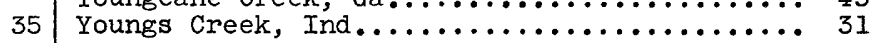

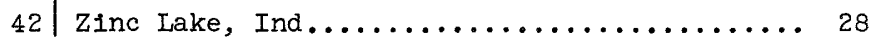


